\title{
Behavioral Phenotyping of an Improved Mouse Model of Phelan-McDermid Syndrome with a Complete Deletion of the Shank3 Gene
}

\author{
Elodie Drapeau,, ${ }^{1,2}$ Mohammed Riad,, ${ }^{1,2}$ Yuji Kajiwara,, ${ }^{1,2}$ and Joseph D. Buxbaum,2,3,4,5,6
}

\section{DOI:http://dx.doi.org/10.1523/ENEURO.0046-18.2018}

\begin{abstract}
${ }^{1}$ Seaver Autism Center for Research and Treatment, Icahn School of Medicine at Mount Sinai, New York, NY 10029, ${ }^{2}$ Department of Psychiatry, Icahn School of Medicine at Mount Sinai, New York, NY 10029, ${ }^{3}$ Department of Genetics and Genomic Sciences, Icahn School of Medicine at Mount Sinai, New York, NY 10029, ${ }^{4}$ Department of Neuroscience, Icahn School of Medicine at Mount Sinai, New York, NY 10029, ${ }^{5}$ Mindich Child Health and Development Institute, Icahn School of Medicine at Mount Sinai, New York, NY 10029, and ${ }^{6}$ Friedman Brain Institute, Icahn School of Medicine at Mount Sinai, New York, NY 10029
\end{abstract}

\begin{abstract}
Phelan-McDermid syndrome (PMS) is a rare genetic disorder in which one copy of the SHANK3 gene is missing or mutated, leading to a global developmental delay, intellectual disability (ID), and autism. Multiple intragenic promoters and alternatively spliced exons are responsible for the formation of numerous isoforms. Many genetically-modified mouse models of PMS have been generated but most disrupt only some of the isoforms. In contrast, the vast majority of known SHANK3 mutations found in patients involve deletions that disrupt all isoforms. Here, we report the production and thorough behavioral characterization of a new mouse model in which all Shank3 isoforms are disrupted. Domains and tasks examined in adults included measures of general health, neurological reflexes, motor abilities, sensory reactivity, social behavior, repetitive behaviors, cognition and behavioral inflexibility, and anxiety. Our mice are more severely affected than previously published models. While the deficits were typically more pronounced in homozygotes, an intermediate phenotype was observed for heterozygotes in many paradigms. As in other Shank3 mouse models, stereotypies, including increased grooming, were observed. Additionally, sensory alterations were detected in both neonatal and adult mice, and motor behavior was strongly altered, especially in the open field and rotarod locomotor tests. While social behaviors measured with the three-chambered social approach and male-female interaction tests were not strongly impacted, Shank3-deficient mice displayed a strong escape behavior and avoidance of inanimate objects in novel object recognition, repetitive novel object contact, marble burying, and nest building tasks, indicating increased novelty-induced anxiety. Similarly, increased freezing was observed during fear conditioning training and amygdala-dependent cued retrieval. Finally, deficits were observed in both initial training and reversal in the
\end{abstract}

\section{Significance Statement}

Phelan-McDermid syndrome (PMS), caused by happloinsufficiency of Shank3, is a severe and complex neurodevelopmental disorder. This study investigates the behavioral consequences of a disruption of all Shank3 isoforms in neonatal and adult mice using a detailed battery of tests tailored to investigate core symptoms and usual comorbidities of PMS. We found that our new model is more severely affected than previously published mouse models with only partial deletions of Shank3 and more closely recapitulates symptoms of PMS, thus providing improvements for both construct and face validity. Our results highlight the significance of using a mouse model with a complete deletion of Shank3 for studying mechanisms underlying autism spectrum disorder (ASD) and PMS, carrying preclinical studies and testing test novel therapeutic approaches. 
Barnes maze and in contextual fear testing, which are memory tasks involving hippocampal-prefrontal circuits. In contrast, working memory in the $\mathrm{Y}$-maze spontaneous alternation test was not altered. This new mouse model of PMS, engineered to most closely represent human mutations, recapitulates core symptoms of PMS providing improvements for both construct and face validity, compared to previous models.

Key words: 22q13; autism spectrum disorder; behavior; mouse model; Phelan-McDermid syndrome; Shank3

\section{Introduction}

Phelan-McDermid syndrome (PMS) is a rare and complex neurodevelopmental disorder that manifests with global developmental delay, mild dysmorphic features, motor deficits, variable degrees of intellectual disability (ID), and absent or delayed speech. Additionally, autism spectrum disorder (ASD), epilepsy, attention deficits, and recurrent medical comorbidities are common in patients with PMS (Phelan and McDermid, 2012; Betancur and Buxbaum, 2013; Soorya et al., 2013; Sarasua et al., 2014a). Recent studies show that PMS is emerging as one of the most frequent and penetrant monogenic causes of autism and ID (Sykes et al., 2009; Betancur and Buxbaum, 2013; Soorya et al., 2013; Leblond et al., 2014).

Despite overlapping etiologies between patients, there is a tremendous heterogeneity in the expression and severity of the phenotype (Cusmano-Ozog et al., 2007; Dhar et al., 2010; Phelan and Betancur, 2011; Soorya et al., 2013). This is no doubt in part due to the complex nature of in the genetic etiology of PMS (De Rubeis et al., 2018). While a large body of data indicates that haploinsufficiency of SHANK3 is the key contributor for the neurobehavioral manifestations of PMS, it can be caused by a variety of genetic rearrangements including unbalanced translocations, ring chromosome 22, terminal deletions (ranging from deletions of just SHANK3 to large deletions of up to $9 \mathrm{Mb}$ ), and interstitial deletions or point mutation within the SHANK3 gene (Durand et al., 2007; Moessner et al., 2007; Sykes et al., 2009; Bonaglia et al., 2011; Phelan and McDermid, 2012; Soorya et al., 2013; Leblond et al., 2014; De Rubeis et al., 2018).

Genotype-phenotype analyses have shown positive correlations between the size of the deletion and the number and/or severity of some phenotypes (Luciani et al., 2003; Dhar et al., 2010; Bonaglia et al., 2011; Soorya et al., 2013; Sarasua et al., 2014b). However,

Received January 24, 2018; accepted May 28, 2018; First published June 05, 2018.

The authors declare no competing financial interests.

Author contributions: J.D.B. and E.D. designed research; E.D., M.R., and Y.K. performed research; E.D. analyzed data; E.D. wrote the paper.

This work was supported by the Beatrice and Samuel A. Seaver Foundation and by National Institutes of Health Grants R01MH093725 and R01MH101584.

Acknowledgements: We thank Jacqueline Crawley for her help all along this study and her helpful comments on this manuscript and to Jill Silverman for reviewing our results. We also thank Dr. Nikolaos Daskalakis for all our helpful discussions and his help with data analysis.

Correspondence should be addressed to Joseph D. Buxbaum, Icahn School of Medicine at Mount Sinai, 1 Gustave L. Levy Place, New York, NY 10029. E-mail: joseph.buxbaum@mssm.edu.

DOI:http://dx.doi.org/10.1523/ENEURO.0046-18.2018

Copyright @ 2018 Drapeau et al.

This is an open-access article distributed under the terms of the Creative Commons Attribution 4.0 International license, which permits unrestricted use, distribution and reproduction in any medium provided that the original work is properly attributed. findings on specific clinical variables have not been consistent across studies. Importantly, it has become clear that indels or point mutations that impact SHANK3 alone can lead to all of the neurobehavioral phenotypes of PMS (De Rubeis et al., 2018). The SHANK3 gene has multiple promoters and is alternatively spliced and the number of Shank3 isoforms can be extensive (Maunakea et al., 2010; Benthani et al., 2015). Some de novo microdeletions or mutations of SHANK3 can therefore affect some but not other SHANK3 isoforms. The genetic heterogeneity of PMS underscores the importance of studying a wide range of mutations and deletions. SHANK3 (ProSAP2) is a major scaffolding protein that forms a key structural part of the postsynaptic density of excitatory glutamatergic synapses. SHANK3 contains multiple proteinprotein interaction domains that each mediates specific protein-protein interactions at synapses. Moreover, the expression and alternative splicing of Shank3 isoforms or even their subcellular distribution has been shown to be cell-type specific, activity dependent as well as regionally and developmentally regulated (Wang et al., 2014) raising the possibility that differing SHANK3 isoforms may play distinct roles in synaptic developmental and function and hence may make distinct contributions to the pathobiology of PMS.

More than a dozen isoform-specific Shank3 mouse models have been independently generated (Table 1). As expected, these models shared some similarities but also showed significant differences in molecular, synaptic, and behavioral phenotypes. Depending on the targeted exons, alterations have been reported in motor functions, social interactions, ultrasonic vocalizations, repetitive grooming, cognitive functions, and anxiety. However, very high variability has been observed regarding the presence or the intensity of such impairments across several types of Shank3-deficient models or even across different cohorts of the same model. These models are based on exonic deletions that have not been reported in human and do not reflect the vast majority of known PMS cases, which are caused by deletions affecting all SHANK3 isoforms. There was therefore an urgent need to develop an animal model with broader construct validity for PMS to fully understand the consequences of a complete deletion of SHANK3 across the range of behavioral phenotypes, which we achieved through a deletion of exons 4-22.

Interestingly, as our work was progressing, a completely independent mouse model, similarly targeting exons 4-22, was reported (Wang et al., 2016b). These mice highlight cortico-striatal circuit abnormalities and demonstrate a behavioral phenotype that resemble features of PMS. We therefore decided to conduct a comprehensive and behavioral evaluation of our mouse model evaluating many more phenotypes relevant to PMS and ASD. Criti- 


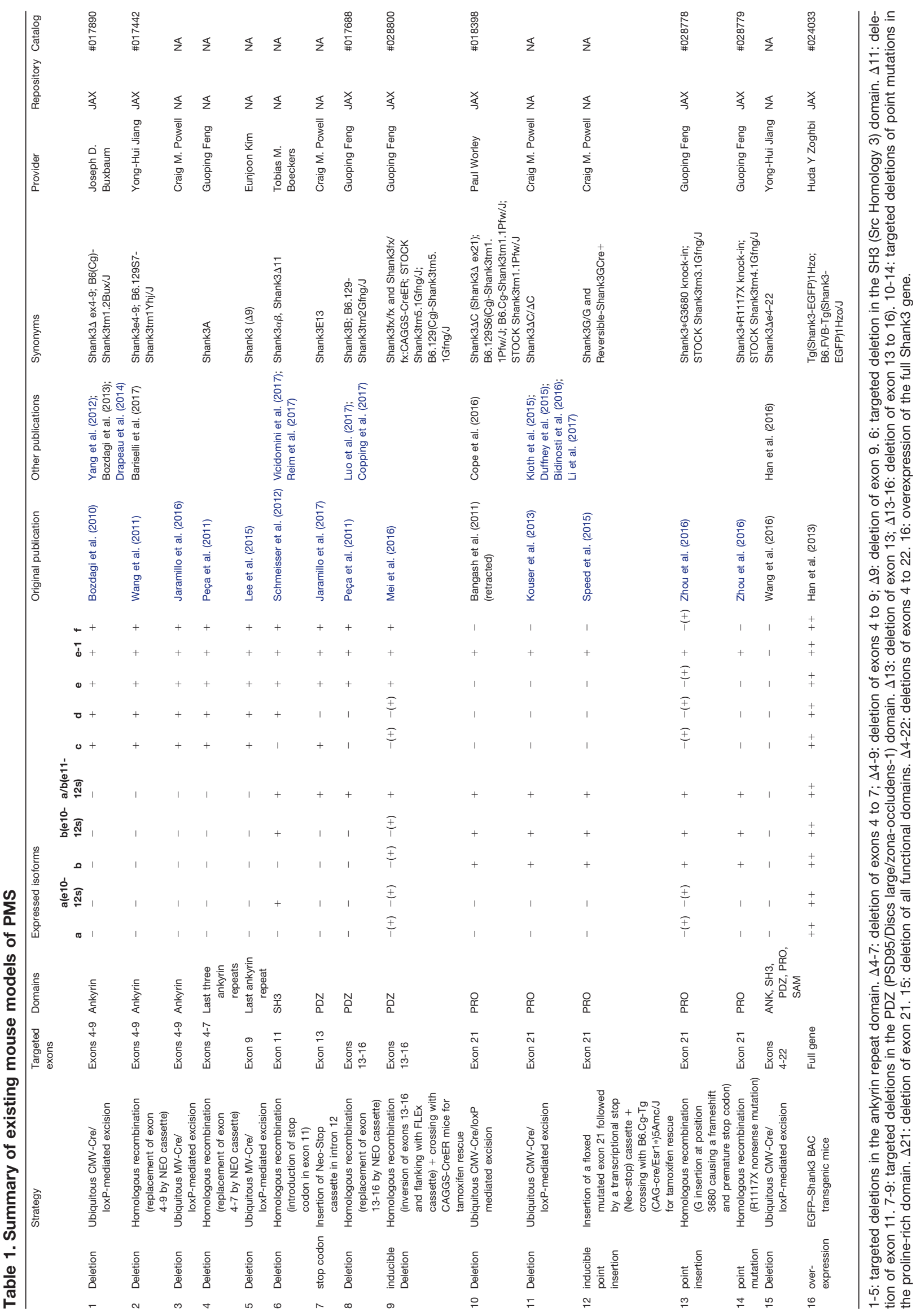


cally, our findings complement and supplement the observations made by the Jiang group with many results clearly confirmed across two independent laboratories, as well as unique analyses in each study.

\section{Materials and Methods}

\section{Generation of inbred strains of Shank ${ }^{\mathbf{4 - 2 2}}$-deficient animals}

All animal procedures were approved by the Institutional Animal Care and Use Committee of the Icahn School of Medicine at Mount Sinai. A Shank $3^{\Delta 4-22}$ mouse line with a complete disruption of the Shank3 gene was generated at Ozgene by retargeting Bruce 4 C57BL/6 embryonic stem cells from a previously published mouse. A third loxP site was inserted immediately downstream of exon 22 in addition of the 2 pre-existing loxP sites flanking exons 4 and 9 (Fig. 1A). To generate the mice used in the present study, the floxed allele was excised by breeding with a CMV-Cre transgenic line ( $\mathrm{Tg}(\mathrm{CMV}$-cre) $1 \mathrm{Cgn}$, The Jackson Laboratory, \#006054) resulting in a deletion of exons 4-22 and therefore a constitutive disruption of all the Shank3 murine isoforms. Both the floxed and deleted mouse strains are available at The Jackson Laboratory Repository (Shank $3^{\Delta 4-22}$ floxed strain: JAX Stock No. 032158; Shank3 ${ }^{\Delta 4-22}$ deleted strain: JAX Stock No. 032169; http://jaxmice.jax.org/query).

The colony was maintained on a pure C57BL/6Tac background (Taconic). Heterozygous mice were mated to generate litters consisting of three genotypes, wildtype (WT), heterozygote (Het), and knock-out (KO). Mice were weaned at $21 \mathrm{~d}$ of age, and at least one littermate from each genotype were group housed in standard plastic cages of three to five littermates per cage. Standard rodent chow and tap water were available ad libitum. The colony room was maintained on a $12 / 12 \mathrm{~h}$ light/dark cycle with lights on at 6 A.M. at a constant temperature of $21-22^{\circ} \mathrm{C}$ and $55 \%$ humidity. All animal procedures were performed in accordance with the Icahn School of Medicine at Mount Sinai animal care committee's regulations.

\section{Genotyping}

The confirmation of the deletions of all Shank3 isoforms was performed by RT-PCR. All the animals included in this study were genotyped using tail samples collected at the time of weaning. Additionally, the genotype of all the adult animals was confirmed using a supplementary biopsy at the end of the behavioral testing. Mouse tail snips were collected by dissecting $0.2 \mathrm{~cm}$ of tail between postnatal days 15 and 21. Tails were digested, genomic DNA isolated and purified using the QIAGEN DNAeasy kit (QIAGEN) according to the manufacturer's instructions. After the extraction, $2.0 \mu \mathrm{l}$ of DNA in buffer containing $\sim 250-$ $400 \mu \mathrm{g}$ of DNA was amplified by PCR using standard PCR methods and a combination of three primers designed inside and outside the deleted region to identify both the wild-type and $\Delta \mathrm{e} 4-22$ alleles (Fig. 1; Extended Data Fig. 1-1; P1-KO: TGAGACCAGAGTTGTTAGGATTTG, P2-WT: AGATGGCTCAGCCAGGTAAG, P3-Common AGATGGCTCAGCCAGGTAAG). The P1-P3 primer pair produced a $490-b p$ band identifying the $\Delta \mathrm{e} 4-22$ allele, while the P2-P3 primer pair amplified a 390-bp band from the wild-type allele. Denaturing, annealing, and extension steps were performed using $94^{\circ} \mathrm{C}$ for 3 min, 35 cycles of $94^{\circ} \mathrm{C}$ for $30 \mathrm{~s}, 62^{\circ} \mathrm{C}$ for $45 \mathrm{~s}, 45^{\circ} \mathrm{C}$ for $30 \mathrm{~s}$, and for 1 cycle $72^{\circ} \mathrm{C}$ for $4 \mathrm{~min}$. The PCR products were run on a $1.5 \%$ agarose gel and stained with ethidium bromide.

\section{Immunoblotting}

Postsynaptic density (PSD) fractions were prepared as follows. Hemibrains of wild-type, heterozygous, and homozygous Shank $3^{\Delta 4-22}$ mice were homogenized in 2-[4(2-hydroxyethyl)piperazin-1-yl]ethanesulfonic acid (HEPES)-A containing $4 \mathrm{mM}$ HEPES, pH 7.4, $0.32 \mathrm{M}$ sucrose, and Protease Inhibitor Cocktail and PhoSTOP Phosphatase Inhibitor Cocktail (both from Roche). Nuclear fractions were precipitated by centrifuging twice at $700 \times g$ for $15 \mathrm{~min}$, and the resulting supernatants were further centrifuged at 21,000 $\times g$ for $15 \mathrm{~min}$. The precipitates were resuspended in HEPES-B containing 4 mM HEPES, pH 7.4, Protease Inhibitor Cocktail, and PhoSTOP Phosphatase Inhibitor Cocktail, homogenized, and rotated at $4^{\circ} \mathrm{C}$ for $1 \mathrm{~h}$. The lysates were centrifuged at $32,000 \times g$ for $20 \mathrm{~min}$ and washed twice with HEPES-C containing 50 mM HEPES, pH 7.4, 0.5\% Triton X-100, Protease Inhibitor Cocktail, and PhoSTOP Phosphatase Inhibitor Cocktail. Finally, PSD fractions were resuspended in HEPES-C containing $1.8 \%$ sodium dodecyl sulfate (SDS) and $2.5 \mathrm{M}$ urea. Fifty micrograms of PSD fraction was loaded to $4-12 \%$ SDS-PAGE (PAGE gel, Invitrogen), transferred to polyvinylidene fluoride membrane and immunoblotted with either the N367/62 anti-Shank3 antibody directed against an epitope in the SH3 domain (UC Davis/NIH NeuroMab Facility) or the H160 anti-Shank3 antibody directed against amino acids 1431-1590 mapping near the C terminus of isoform 2 of Shank 3 (sc-30193, Santa Cruz Biotechnology). For $\beta$ III-tubulin, the membrane was stripped and immunoblotted with an anti- $\beta$ III-tubulin antibody (Abcam).

\section{RT-PCR isoform analysis}

Total RNA from hemibrains of wild-type and homozygous Shank $3^{\Delta 4-22}$ mice was isolated using the TRIzol method (Invitrogen, ThermoFisher Scientific). Reverse transcription was performed with SuperScript III first-strand synthesis system (Invitrogen, ThermoFisher Scientific). DNA was amplified by PCR using standard PCR methods and the following primers ass described previously (Wang et al., 2014). Shank3a forward: ACGAAGTGCCTGCGTCTGGAC, Shank3a reverse: CTCTTGCCAACCATTCTCATCAGTG; Shank3b forward: GTAGCCACCTCTTGCTCACAT, Shank3b reverse: TTGCCAACCATTCTCATCAGT; Shank3c forward: CTTCTT CACTGGCAATCCTTG, Shank3c reverse: CAGTGTAGTGGCGGAAGAGAC; Shank3d forward: AGGGTCACGACTGTTTCTTAGC, Shank3d reverse: TGTGGGTGTAAACTCCTCAATG; Shank3e forward: GTACCTGGGTCTGGGTGCTITA, Shank3e reverse: AACTGCCAGGATCTCATCCA.

\section{Behavioral overview}

Multiple cohorts were used for behavioral testing. The first cohort consisted of 54 newborn mice (14 WT, 30 Het and $10 \mathrm{KO}$ ) from 10 independent litters. The second 


\section{A}

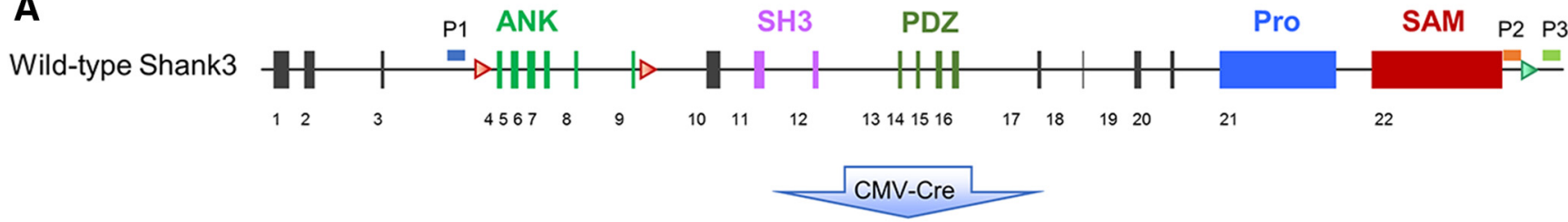

Shank3 $\Delta 4-22$ -

B

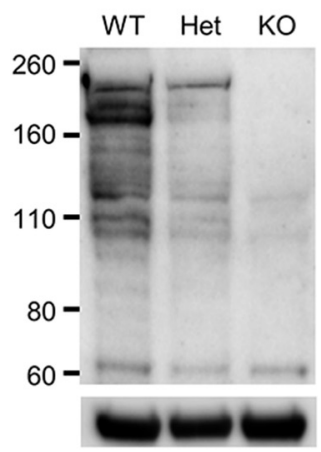

$\mathrm{SH} 3 \mathrm{Ab}$

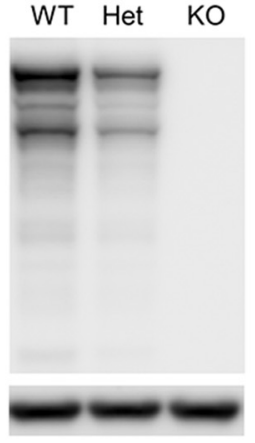

C-terminal $\mathrm{Ab}$

\section{Genotype distribution at weaning}

D

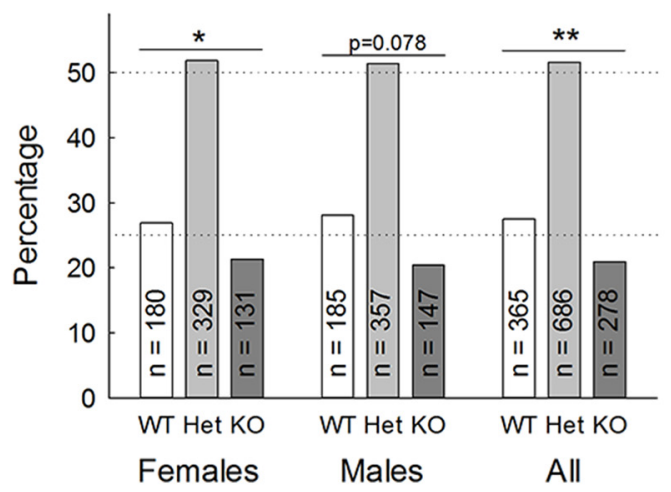

Adult survival curve

E

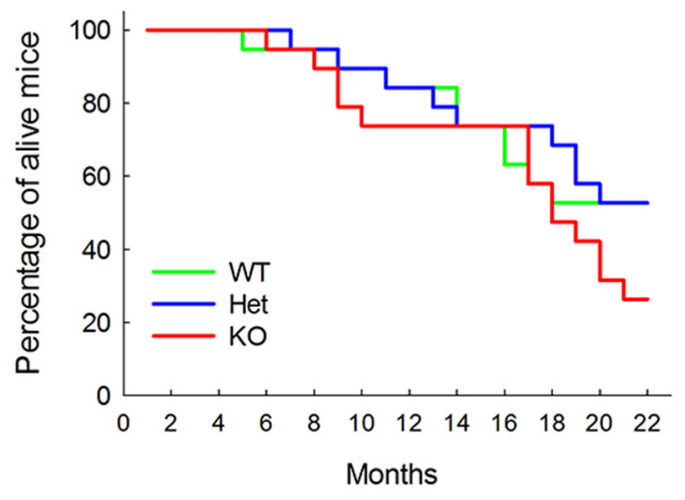

Figure 1. Generation and validation of a knock-out mice with a complete deletion of Shank3. $\boldsymbol{A}$, Schematic design for generation of a Shank $3^{\Delta 4-22}$ complete knock-out mouse using a Cre-loxP strategy. Bruce4 C57BL/6 embryonic stem cells from a previously generated mouse with two LoxP site located upstream exon 4 and downstream exon 9 (top, red triangles) were retargeted to insert an additional LoxP site 155 pb downstream of exon 22 (green triangle). Floxed mice were crossed with CMV-Cre mice to generate ubiquitous deletion of exons 4-22 (bottom). ANK, ankyrin repeats; SH3, Src homology 3 domain; PDZ, PSD/Dlg1/zo-1 domain; Pro, proline-rich domain; SAM, sterile $\alpha$-motif domain. The positions of the PCR primers (P1, P2, P3) for genotyping are indicated. $\boldsymbol{B}$, Expression of Shank3 in PSD fractions. PSD fractions from wild-type, heterozygous, and homozygous mice were subjected to immunoblotting with either the N367/62 anti-Shank3 antibody directed against an epitope in the SH3 domain or the $\mathrm{H} 160 \mathrm{C}$-terminal antibody. Immunoblots show that all Shank3 protein bands are absent in KO brains. The migration of molecular weight markers is shown on the left (in kilodaltons) and an immunoblot for $\beta$ Ill-tubulin as a loading control is shown below. Original full scans of immunoblots are displayed in Extended Data Figure 1-1. C, RT-PCR analysis for specific Shank3 transcripts in Shank $3^{\Delta 4-22}$ mice. Brain-derived mRNAs from wild-type and homozygous mice were subjected to RT-PCR targeting different isoforms. All transcripts were absent in Shank $3^{\Delta 4-22}$ homozygous mice. $\boldsymbol{D}$, Distribution of genotype. A deficit in the number of Shank $3^{\Delta 4-22}$ knockout mice was observed at the time of weaning. $\boldsymbol{E}$, Survival curve of Shank ${ }^{\Delta 4-22}$ wild-type, heterozygous and homozygous mice between 2 and 22 months. WT, wild-type mice; Het, heterozygous mice; KO, homozygous knockout mice. $*: p<0.05, * *: p<0.1$.

cohort consisted of 57 newborn mice (16 WT, 32 Het, and nine KO) from nine independent litters. Cohorts 3 (30 adult male mice, 11 WT, 10 Het, and nine KO) and 4 (27 adult male mice, 11 WT, $10 \mathrm{Het}$, and nine KO) were tested between 3 and 10 months of age according to the schedule described in Table 2. In each adult cohort, all mice were born within two weeks of each other, and generally only one triplet came from any given individual litter of 
Table 2. Cohorts used and order of behavioral testing

\begin{tabular}{llllll}
\hline Cohort 1 (10 litters) - developmental milestones & & & & \\
& & & & Age at \\
& WT & Het & KO & testing \\
All animals & 14 & 30 & 10 & P0-P21 \\
Males & 7 & 16 & 5 & P0-P21 \\
Females & 7 & 14 & 5 & P0-P21
\end{tabular}

Cohort 2 (10 litters) - ultrasonic vocalizations

All animals

Males

Females

Cohorts 3 and 4 - adult behavior

Handling, cage observation, neurological and motor reflexes

15-month weight

20-month weight

Open field

Zero-maze

Y-maze

Beam walking

Grip strength

Gait analysis

Rotarod

Three-chambered social interaction task

Nest building

Novel object

Fear conditioning

Startle response *

Pre-pulse inhibition

Tail flick

Olfactory habituation/dishabituation

Buried food

Social transmission of food preference

Marble burying

Four-object repetitivenovelobject contact task

Male-female social interaction

Barnes maze

$\begin{array}{llll}\text { WT } & \text { Het } & \text { KO } & \begin{array}{l}\text { Age at } \\ \text { testing }\end{array} \\ 16 & 32 & 9 & \text { P6 } \\ 4 & 15 & 6 & \text { P6 } \\ 12 & 17 & 3 & \text { P6 }\end{array}$

\begin{tabular}{|c|c|c|c|c|c|c|}
\hline \multicolumn{3}{|l|}{ Cohort 3} & \multicolumn{4}{|c|}{ Cohort 4} \\
\hline Het & $\mathrm{KO}$ & $\begin{array}{l}\text { Age at } \\
\text { testing }\end{array}$ & WT & Het & $\mathrm{KO}$ & $\begin{array}{l}\text { Age at } \\
\text { testing }\end{array}$ \\
\hline 11 & 9 & P86-P90 & 8 & 9 & 10 & P103-P107 \\
\hline 8 & 6 & P460 & 5 & 7 & 4 & P455 \\
\hline 7 & 2 & P610 & 4 & 5 & 3 & P600 \\
\hline 11 & 9 & P93-P94 & 8 & 9 & 10 & P106-P108 \\
\hline 11 & 9 & P95-P96 & 8 & 9 & 10 & P109-P110 \\
\hline 11 & 9 & P99-P101 & 8 & 9 & 10 & P114-P122 \\
\hline 11 & 9 & P102-P103 & 8 & 9 & 10 & P124-P125 \\
\hline 11 & 9 & P104 & 8 & 9 & 10 & P125 \\
\hline 11 & 9 & P105 & 8 & 9 & 10 & P126 \\
\hline 11 & 9 & P107-P108 & 8 & 9 & 10 & P127 \\
\hline 11 & 9 & P113-P114 & 8 & 9 & 10 & P130-P131 \\
\hline 11 & 9 & P120 & 8 & 9 & 10 & P137 \\
\hline 11 & 9 & P123-P125 & 8 & 9 & 10 & P139-P140 \\
\hline 11 & 9 & P126-P128 & 8 & 9 & 10 & P141-P143 \\
\hline 11 & 9 & P137-P139 & $3 *$ & $4 *$ & $4 *$ & P155-P157 \\
\hline 11 & 9 & P137-P139 & 8 & 9 & 10 & P155-P157 \\
\hline 11 & 9 & P144-P145 & 8 & 9 & 10 & P158-P159 \\
\hline 11 & 9 & P149-P157 & 8 & 9 & 10 & P162-P165 \\
\hline 11 & 9 & P163-P164 & 8 & 9 & 10 & P178 \\
\hline 11 & 9 & P206-P215 & 8 & 9 & 10 & P185-P192 \\
\hline 11 & 8 & P227-P228 & 8 & 9 & 10 & P197 \\
\hline 11 & 8 & P232 & 7 & 9 & 9 & P215 \\
\hline 11 & 8 & P240-P241 & 7 & 9 & 9 & P217-P219 \\
\hline 11 & 7 & P247-P274 & 7 & 9 & 8 & P222-P250 \\
\hline
\end{tabular}

For adult animals, the age indicated corresponds to the average age of the cohort. For each cohort all mice were born within two weeks of each other. *: missing animals due to technical problems during startle recording.

mice. Behavioral experiments were conducted between 9 A.M. and 5 P.M. during the light phase of the $12 / 12 \mathrm{~h}$ light/dark cycle in dedicated testing sound-attenuated rooms. Mice were brought to the front room of the testing area at least half an hour before the start of experiments. All three genotypes were tested on the same day in randomized order by two investigators who were blind to the genotypes. Behavioral tests were conducted in the order and at the ages indicated in Table 2 and included developmental milestones, cage observation, neurologic and motor reflexes, open field, elevated zero-maze, Y-maze, beam walking, grip strength, gait analysis, rotarod, three-chambered social interaction task, nest building, novel object recognition, fear conditioning, pre-pulse inhibition, tail flick, olfactory habituation/dishabituation, buried food, social transmission of food preference, marble burying, four-object repetitive novel object contact task, male-female social interaction, and Barnes maze. Behavioral results are not described in the order they were tested in an effort to ease presentation and interpretation of the data.

\section{Newborn development}

The physical, sensory and motor developmental milestones of neonates were assessed between postnatal days 1 and 21 using a battery of tests adapted from the Fox scale (Fox, 1965; Heyser, 2004). As we had previously observed, a higher rate of postnatal mortality on the first litter, only dams that already had one litter were used for this experiment. To control for litter and avoid nutritional effects the litter size was homogenized and limited to six pups per dam by reducing larger litters and adding excess pups to smaller litters on the morning of postnatal day 1 
where and when possible. At this time, pups were identified by paw tattoo using a nontoxic animal tattoo ink (Animal Identification \& Marking Systems Inc) inserted subcutaneously through a 30-gauge hypodermic needle tip into the center of the paw. Individual pups were removed from the litter and placed on cotton pads in a heated cage under a heating lamp throughout the testing. Each subject was tested at approximately the same time of day. For all the timed tests, a 30-s cutoff was used and nonresponding animal received a score of $30 \mathrm{~s}$. Most responses were considered positive only after they had been observed for two consecutive days.

The physical development was measured by following the weight (postnatal day 1 to 21), eye opening (postnatal days 9 to 20), tooth eruption (postnatal days 7 to 18), the ear development (postnatal day 1 to 9), and the fur development (postnatal days 1 to 14) using the following scales. Eye opening, per eye: $0=$ eye fully closed, $1=$ eye partially opened, 2 = eye full opened, tooth eruption, scored separately for bottom and top incisors: $0=$ incisors not visible, 1 = incisors visible but not erupted, 2 = incisors fully erupted. Ear development, per ear: $0=$ ear bud not detached from the pinna, 1 = ear flap detached from the pinna, ear fully developed on the back of the ear). Fur development: $1=$ bright red, 2 = nude, pink, 3 = nude, gray, 4 = gray, fuzzy on back and shoulder, 5 = black hair on back, gray fuzzy belly, 6 = body fully covered.

Sensory development was assessed using cliff aversion (postnatal days 2 to 14), auditory startle (postnatal days 6 to 18), rooting reflex (postnatal days 2 to 10), ear twitch (postnatal days 7 to 15), and forelimb grasp (postnatal days 4 to 14) using the following measures. For cliff aversion, the subject was placed on the edge of a Plexiglas platform with a $30-\mathrm{cm}$ cliff with its nose and forefeet over the edge. The latency to move away from the edge was recorded. Auditory startle was measured in response to an $80-\mathrm{dB}$ click $30 \mathrm{~cm}$ above the mouse and was considered present when the pup moved immediately after the presentation of the auditory stimulus. For the rooting reflex, the side of the pup's face were bilaterally stimulated with two cotton swabs. The reflex was considered present when the pup crawled forwards pushing the head during the stimulation. For the ear twitch, the ear of the pup was stimulated with the tip of a cotton swab that was previously pulled to form a filament. Both ears were successively stimulated and the test was considered positive when the pup turned its head or jumped in response to the stimulation. The forelimb reflex was tested by gently stimulated the front paws with the loop of a small bended metallic wire. Each front paw was scored separately as follow: 0 = no response to stimulation, 1 = paw folding in response to the stimulation, 2 = paw grasping the wire in response to the stimulation, $3=$ grasp strong enough to hold for at least $1 \mathrm{~s}$ when the wire was lifted up.

Motor development was studied using surface righting (postnatal days 2 to 13), negative geotaxis (postnatal days 2 to 14), air righting (postnatal days 8 to 20), open field crossing (postnatal days 8 to 20), and rod suspension (P11-P20) using the following criteria. The surface righting was measured by the time for pups placed on their back to fully turn with all four paws on the ground. For negative geotaxis, pups were placed head down on a mesh covered plan that was slanted at a $45^{\circ}$ angle, and the latency to either roll down, stay, or turn and move up the slope was recorded. For the air righting, the pup was dropped upside down at a height of $30 \mathrm{~cm}$ over a padded surface. Subjects received a score of 2 if they successfully righted themselves during the fall, 1 if they landed on the side and 0 if they did no turn. The open field crossing was measured by the time to exit a $13 \mathrm{~cm}$ in diameter circle when place on the center of the circle. For the rod suspension, the pups were gently grabbed by the trunk, brought up close to a 3-mm wooden rod $30 \mathrm{~cm}$ above a padded surface and released once they grabbed the rod with their front paws. The latency to stay suspended was recorded.

\section{Physical factors, gross appearance, and spontaneous activity}

Adult animals were handled daily for one week before starting behavioral testing and general health, weight (grams), length (centimeters), physical factors, gross appearance, and spontaneous activity were recording during handling using the following scales.

\section{Physical factor and gross appearance}

Coat appearance: $0=$ ungroomed, $1=$ partially groomed, 2 = semi-groomed, 3 = groomed. Skin color (pinna and footpads): $0=$ pink, $1=$ purple, $2=$ other. Whisker barbering: $0=$ normal, $1=$ abnormally shortened. Patches of missing fur on face or body: $0=$ none, 1 = some, 2 = extensive. Wounding: $0=$ none, 1 = signs of previous wounding, 2 = slight wounds present, $3=$ moderate wounds present, $4=$ extensive wounds present. Body tone when both sides of the mouse are compressed between thumb and index finger: $0=$ flaccid, no return of cavity to normal, $1=$ slight resistance, $2=$ extreme resistance. Palpebral closure: $0=$ eyes wide open, 1 = eyes half open, 2 = eyes closed. Spontaneous piloerection: $0=$ none, 1 - coat standing on end.

Spontaneous general activity in a $1000-\mathrm{ml}$ jar and after transfer in a regular home cage for 5 min each. Body position: 0 = completely flat, 1 = lying on side, 2 = lying prone, 3 = sitting or standing, $4=$ rearing on hind legs, 5 $=$ repeated vertical leaping. Spontaneous activity: $0=$ none, resting, 1 = casual scratch, groom, slow movement, 2 = vigorous scratch, groom, moderate movement, $3=$ vigorous, rapid/dart movement, $4=$ extremely vigorous, rapid/dart movement. Respiration rate: $0=$ gasping, irregular, 1 = slow, shallow, $2=$ normal, 3 = hyperventilation. Tremor: $0=$ none, $1=$ mild, $2=$ marked. Urination: $0=$ none, $1=$ little, $3=$ moderate amount, $4=$ extensive . Defecation: number of fecal boli. Transfer arousal: $0=$ coma, 1 = prolonged freeze, then slight movement, $2=$ brief freeze, then active movement, $3=$ no freeze, stretch attends, $4=$ no freeze, immediate movement (manic). Gait: $0=$ normal, $1=$ fluid but abnormal, $2=$ slow and halting, 3 = limited movement only, $4=$ incapacity. Pelvic elevation: $0=$ markedly flattened, $1=$ barely touches, $2=$ normal $(3 \mathrm{~mm}$ elevation), $3=$ elevated (more than $3 \mathrm{~mm}$ elevation). Tail elevation: $0=$ dragging, $1=$ horizontally 
extended, $2=<30^{\circ}$ elevation, $3=30-60^{\circ}$ elevation, $4=$ $60-90^{\circ}$ elevation.

\section{Motor testing}

Gait analysis

Motor coordination and gait patterns was observed as the subject was allowed to run the length of an elevated runway (dimensions: $152 \mathrm{~cm}$ long $\times 10 \mathrm{~cm}$ wide) lined with white paper (Carter et al., 2001). After three training runs, the subject's paws were coated in nontoxic paint (different colors for hind and front paws) to record paw prints on two consecutive runs. The record displaying the clearest prints and most consistent gait for analysis of $50 \mathrm{~cm}$ was chosen to measure sway (mean distance between left and right paws), stride (mean distance between same side front and hind paws) and diagonal stance (mean distance between diagonally opposed front and hind paws).

\section{Open field}

Mice were tested in an open field $(45 \times 45 \mathrm{~cm})$ virtually divided into central and peripheral regions. Animal activity was recorded by video tracking (Noldus Ethovision). Each mouse was allowed to explore the apparatus for $60 \mathrm{~min}$. The distance traveled, the number of rears and revolutions, the number of grooming bouts and cumulative grooming time, the number of head shaking or twitches, the number of entries in the center, and the time spent in the central and peripheral regions were recorded. Measures were recorded in 10-min intervals.

\section{Rotarod}

Motor coordination, endurance and learning was assessed in the Rotarod test (Omnitech Electronics Inc). Mice were placed on an elevated accelerating rod $(3 \mathrm{~cm}$ in diameter) for three trials per day on two consecutive days. Each trial lasted for a maximum of 5 min, during which the Rotarod underwent a linear acceleration from 4 to $40 \mathrm{rpm}$. A 20-min interval was used between trials to avoid fatigue. Animals were scored for their latency to fall.

\section{Beam walking}

Subtle deficits in fine motor coordination and balance that might not be detected by other motor tests were assessed by the beam walking assay in which the mouse had to walk across an elevated horizontal wood beam (100 $\mathrm{cm}$ long, $1 \mathrm{~m}$ above bedding) to a safe dark box (Carter et al., 2001). Subjects were placed near one end in bright light, while the far end with the dark box was placed in darkness, providing motivation to cross. Performance was quantified by measuring the latency to start crossing, the time to reach the dark box or the time to fall, the total distance traveled and the number of paw slips or incomplete falls (mice able to climb back on the rod). Animals were successively trained on three different beams: 1 inch, $1 / 2$ inch and $1 / 4$ inch diameter and scored on four consecutive trials per beam with $1 \mathrm{~min}$ of rest between trials on the same beam and 20-30 min between each beam. Mice that did not reach the box after 2 min were gently placed inside the box and allowed to stay inside for $1 \mathrm{~min}$.

\section{Righting reflex}

The subject was grasped by the nape of the neck and base of the tail, inverted so back faced down, and re- leased $30 \mathrm{~cm}$ above subject's home cage floor. Righting ability was scored as follow: $0=$ no impairment, $1=$ lands on side, 2 = lands on back, 3 = fails to right even when placed on back on the floor.

\section{Hindlimb placing}

Subject was lowered by the base of the tail until it grasped a horizontal wire grid with both forepaws. The grid was rotated to vertical and the tail was released. Mice were evaluated over three trials, 3 min apart for their latency to fall or latency to pull body on the grid and the ability to place hind paws was scored as follow: $0=$ grabs but falls, $1=$ grabs but hangs, $3=$ grabs and pulls body onto grid. Maximum cutoff was $60 \mathrm{~s}$.

\section{Hanging}

The subject, held from the base of the tail, was allowed to grasp a wooden rod with both forepaws, rotated to horizontal and release. Test was repeated three times with a 3-min interval between trials and a 60-s maximum cutoff. Both the latency to fall and overall performance scored as follow were recorded: $0=$ does not grasp, $1=$ grasps but falls immediately, 2 = grasps but then falls off, $3=$ grasps and stays on for $60 \mathrm{~s}$.

\section{Negative geotaxis}

The subject was placed on a wire mesh grid and the grid was lift vertically, with subject facing down. Test was repeated three times with a 3-min interval between trials and a 60-s maximum cutoff. Both the latency to fall and overall performance scored as follow were recorded: $0=$ falls off, 1 = does not move, 2 = moves but does not turn, 3 = turns but does not climb, $4=$ turns and climbs up.

\section{Inverted screen}

The subject was placed on a grid screen. The grid was waved lightly in the air, then inverted $60 \mathrm{~cm}$ over a cage with soft bedding material. Mice were tested only one time with a 60-s maximum cutoff, and the latency to fall was recorded.

\section{Grip strength}

Forelimb muscle strength and function was evaluated with a strength meter (Ametek). This test relies on the instinctive tendency of mice to grasp an object with their forelimbs. The animal was pulled backward gently by the tail, while grasping a pull bar connected to a tension meter and the force at the moment when the mouse lost its grip was recorded as the peak tension. Test was repeated three times with a 3-min interval between trials. Each trial consisted in five attempts in quick successions for which the best value was recorded therefore increasing the chances that the measure will accurately reflect maximum strength. The mean of three trials and the largest value from all trials were used as parameters.

\section{Sensory testing}

\section{Sensory reflexes}

Sensory abilities were evaluated through the reflex response to several sensory modalities using the following scales. Pinna reflex in response to a gentle touch of the auditory meatus with a cotton-tipped applicator repeated three times with a 10- to 15-s interval: $0=$ none, $1=$ 
active retraction, moderately brisk flick, 2 = hyperactive, repetitive flick. Corneal reflex in response to a gentle puff of air repeated three times with a 10- to 15-s interval: $0=$ no eye blink, 1 = active eye blink, 2 = multiple eye blink. Toe pinch normal retraction reflexes in all four limbs when lightly pinching each paw successively by applying a gentle lateral compression with fine forceps while the mouse is lifted by its tail so the hind limbs are clear of the table. Score is cumulative of four limbs: $0=$ no retraction, $1=$ active retraction, 2 = repetitive retractions. Preyer reflex in response to a $90-\mathrm{dB}$ click $30 \mathrm{~cm}$ above mouse repeated three times with a 10- to 15 -s interval: $0=$ None, 1 = Preyer reflex (head twitch), $2=$ jump $<1 \mathrm{~cm}, 3=$ jump $>1 \mathrm{~cm}$

\section{Tail flick test}

The automated tail flick test (Omnitech Electronics Inc) was used to assess nociceptive threshold. Awake mice were placed in a contention tube to limit movement with their tail resting on the groove of a heating panel. When the mice were calm, a narrow heat producing beam was directed at a small discrete spot $\sim 15 \mathrm{~mm}$ from the tip of the tail. When the subject's tail was removed from the beam, an automatic timer recorded the latency. The test was repeated five times with a 3-min interval between each trial. The latency of the mice to flick their tail was recorded and the two trials with the shorter latencies were discarded since the tail is not always fully in the beam and this is often an outlier.

\section{Acoustic startle response and pre-pulse inhibition of startle}

Subjects were placed in isolation boxes outfitted with accelerometers to measure magnitude of subject movement (Med Associates). After 5 min of acclimation mice were first tested for acoustic startle response. Mice were presented with six discrete blocks of six trials over $8 \mathrm{~min}$, for a total of thirty-six trials. The trials consisted in six responses to no stimulus (baseline movement), six responses to $40-\mathrm{ms}$ sound bursts of $74 \mathrm{~dB}$, six responses to $40-\mathrm{ms}$ sound bursts of $78 \mathrm{~dB}$, six responses to $82-\mathrm{ms}$ sound bursts of $100 \mathrm{~dB}$, five responses to $40-\mathrm{ms}$ sound bursts of $86 \mathrm{~dB}$, and six responses to $40-\mathrm{ms}$ sound bursts of $92 \mathrm{~dB}$. The six trials type were presented in pseudorandom order such that each trial type was presented once within a block of six trials. Mice were then tested for pre-pulse inhibition of startle. They were presented with seven discrete blocks of trials of six trials over $10.5 \mathrm{~min}$ for a total of 42 trials. The trials consisted in six response to no stimulus (baseline movement), six startle response to a 40-ms, 110-dB sound burst, six prepulse inhibition trials where the $110-\mathrm{dB}$ tone was preceded by a $20-\mathrm{ms} 74-\mathrm{dB}$ tone $100 \mathrm{~ms}$ earlier, six prepulse inhibition trials where the $110-\mathrm{dB}$ tone was preceded by a 20-ms 78-dB tone 100 ms earlier, six prepulse inhibition trials where the 110-dB tone was preceded by a $20-\mathrm{ms} 82-\mathrm{dB}$ tone $100 \mathrm{~ms}$ earlier, six prepulse inhibition trials where the $110-\mathrm{dB}$ tone was preceded by a $20-\mathrm{ms} 86-\mathrm{dB}$ tone $100 \mathrm{~ms}$ earlier and six prepulse inhibition trials where the 110-dB tone was preceded by a 20-ms $92-\mathrm{dB}$ tone $100 \mathrm{~ms}$ earlier. The seven trial types were presented in pseudorandom order such that each trial type was presented once within a block of seven trials. Startle amplitude was measured every $1 \mathrm{~ms}$ over a 65-ms period, beginning at the onset of the startle stimulus. The intertrial interval was $10-20 \mathrm{~s}$. The maximum startle amplitude over this sampling period was taken as the dependent variable. A background noise level of $70 \mathrm{~dB}$ was maintained over the duration of the test session.

\section{Visual acuity}

Visual acuity was tested using the visual placing test that takes advantage of the forepaw-reaching reflex: the mouse was held by its tail $\sim 20 \mathrm{~cm}$ above the surface and progressively lowered. As it approaches the surface, the mouse should expand its forepaws to reach the floor. The test was repeated three times with a 30-s interval and the forepaw reaching reflex was quantified as the percentage of forepaw-reaching episodes that did not involve the vibrissae and/or nose touching the surface before the forepaws.

\section{Buried food test}

The buried food test (Yang and Crawley, 2009) measures how quickly an overnight-fasted animal can find a small piece of familiar palatable food, that is hidden underneath a layer of bedding using olfactory clues. Fruit Loops (Kellog's) were used as familiar food. For three consecutive days before the test, three to four pieces were offered to the subjects to make sure it was highly palatable for all the subjects. At 18-24 h before the test, all chow pellets were removed from the subjects' home cages. The water bottle was not removed. On the testing day, the subject was placed in a clean cage $(28 \mathrm{~cm}$ long $\times 18 \mathrm{~cm}$ wide $\times 12 \mathrm{~cm}$ high) containing $3 \mathrm{~cm}$ deep of clean bedding and the subject was allowed to acclimate to the cage for $10 \mathrm{~min}$. While the subject was temporary placed in an empty clean cage, four to five pieces of Fruit Loops were buried $\sim 1 \mathrm{~cm}$ beneath the surface of the bedding, in a random corner of the cage and the bedding surface was smoothed out. The subject was placed back in the testing cage and given $15 \mathrm{~min}$ to retrieve and eat the hidden food. Latency to find the food was recorded. If a subject did not find the food, 15 min was recorded as its latency score and the food was unburied and presented to the mouse by the experimenter to make sure that it was palatable for the mouse. At the end of testing, subjects were hold in a temporary cage until all animals from the same home cage were tested.

\section{Olfactory habituation and dishabituation}

This test consisted of sequential presentations of different nonsocial and social odors in the following order: water, lemon extract (McCormick; 1:100 dilution), banana extract (McCormick; 1:100 dilution), unfamiliar males and unfamiliar females (Yang and Crawley, 2009). Lemon and banana solutions were freshly prepared everyday using distilled water. Social odors were obtained from cages of unfamiliar C56BL/6 mice of the same and opposite sex as the subject which have not been changed for at least $3 \mathrm{~d}$ and were maintained outside of the experimental testing room. Social odor stimuli were prepared by wiping a cotton swab in a zigzag motion across the cage. The 
subject was placed in a clean bedding-covered testing cage covered with the cage grid. A clean dry applicator $(10-\mathrm{cm}$ cotton swab) was inserted through the cage grid water bottle hole and the animal was allowed to acclimate for $30 \mathrm{~min}$ to reduce novelty-induced exploratory activity during the olfaction test. Each odor (or water) was presented in three consecutive trials for a duration of $2 \mathrm{~min}$. The intertrial interval was $1 \mathrm{~min}$, which is about the amount of time needed to change the odor stimulus. At the end of testing, subjects were hold in a temporary cage until all animals from the same home cage were tested. The test was videotaped and subsequently scored. Sniffing and direct interaction time (touching, biting, climbing the applicator) were quantified separately.

\section{Social tests}

Three-chambered social approach test

Sociability and preference for social novelty and social recognition were tested in a three-chambered apparatus (Nadler et al., 2004). The subject mouse was first placed in the central, neutral chamber and allowed to explore for 10 min with all doors closed. Next, doors were opened and the mouse was allowed to freely explore the three empty chambers for an additional 10 min. Lack of side preference was confirmed during this habituation. The subject was then temporary placed in a holding cage while two empty wire cages which allow for olfactory, visual, auditory, and tactile contacts but not for sexual contact or fighting containing either an inanimate object (black cone) or a male mouse were placed in each of the testing chambers and the subject was returned to the apparatus for a 10-min testing phase. Adult mice from the same strain that was previously habituated to the wire cup and did not exhibit aggressive behaviors but had no previous contact with the subject were used for unfamiliar mice. Unfamiliar mice were not used more than twice a day with at least $2 \mathrm{~h}$ before two tests. At the end of testing, subjects were hold in a temporary cage until all animals from the same home cage have been tested. The side position of the interacting animal and the object was randomly determined. All the sessions were videotracked (Noldus Ethovision) and the amount of time spent in each chamber, close to the holding cages or in direct interaction with the holding cage was automatically calculated.

\section{Male-female social interaction}

Male-female social interactions were evaluated in in a regular clean cage during a 10-min test session as previously described (Scattoni et al., 2011). Each subject male was paired with an unfamiliar estrus C57BL/6J female under low light (10 lux) conditions. A total of 20 females were used for this test allowing to avoid to reuse the same female more than twice on the same day. The sessions were videotaped and ultrasonic vocalizations were recorded using an ultrasonic microphone with a $250-\mathrm{kHz}$ sampling rate (Noldus Ultravox XT) positioned $10 \mathrm{~cm}$ above the cage. The entire set-up was installed in a sound-attenuating room. Videos from the male subjects were subsequently manually scored to quantify (number of events and total time of male to female nose-to-nose sniffing, nose-to-anogenital sniffing, and sniffing of other body regions. Ultrasonic vocalizations were played back and spectrograms were displayed using the Ultravox XT software and ultrasonic vocalizations were manually quantified.

\section{Social transmission of food preference}

The social transmission of food preference is a test of olfaction memory that involves a social component through the use of a demonstrator mouse (Wrenn et al., 2003). The demonstrator mouse is a conspecific mouse of same sex and similar age that was labeled by bleaching before testing. To minimize neophobia during the experiments, both subjects and demonstrator mice were habituated to eat powdered rodent chow (AIN-93M, Dyets, Inc.) from 4-Oz (113.40-g) glass food jar assemblies (Dyets, Inc.). This habituation was performed for $48 \mathrm{~h}$ in the mice home cage while the regular pellet chow was removed from the cages. After the habituation, both subject mice and demonstrator mice were food deprived for 18-24 $\mathrm{h}$ before testing with free access to water. The test was divided into three phases.

Demonstrator exposition During the first phase the demonstrator was presented with a jar of powder food mixed with either $1 \%$ cinnamon or $2 \%$ cocoa. The flavor was randomly assigned to the demonstrators so half of them received the cocoa flavored food while the other half received the cinnamon flavored food. Each demonstrator was used only once a day. The demonstrators were allowed to eat the flavored food for $1 \mathrm{~h}$. The jars were weighed before and after presentation to the demonstrators. The criterion for inclusion in the experiment was consumption of $0.2 \mathrm{~g}$ or more.

Interaction phase After eating the flavored food, a demonstrator was placed in an interaction cage with the observer subject mouse and mice were allowed to freely interact for $30 \mathrm{~min}$.

Choice phase Immediately after the interaction phase, the observer mouse was placed in a clean cage and presented with one jar containing the flavor of food eaten by the demonstrator (cued) and another jar containing the other flavor and given $1 \mathrm{~h}$ to freely explore the jar and eat. The demonstrator flavor and the position of the jar (front or back of the cage) was randomly assigned.

All phases were videotaped and food jars were weighed before and after the sessions to determine the amount of food eaten. At the end of testing, demonstrators and observers were hold in temporary cages until all animals from the same home cage had been tested. Video recordings from the interaction phase were used to score the number and total time of sniffing bouts from the observer to the nose or head of the demonstrator. Video recordings from the choice phase were used to score the total time spent in interaction with each food jar (mouse observed in the top of the jar with nose in jar hole).

\section{Avoidance, escape behavior, and hyper-reactivity}

Object avoidance and escape behavior was observed in several tests initially designed to assess other behaviors, including the novel object recognition, the marble burying, and the nest building. 


\section{Novel object recognition}

The novel object test for object recognition and memory takes place in an opacified open field arena $(45 \times 45 \mathrm{~cm})$. The test involves a set of two unique novel objects, each about the size of a mouse, constructed from two different materials and nonuniform in shape. The test consisted of one 10-min habituation session, a 5-min familiarization session and a 5-min recognition test, each videotracked (Noldus Ethovision). During the habituation, animals were allowed to freely explore an empty open field. At the end of the session, they were removed from the open field and place in a temporary clean holding cage for about 2 min. Two identical objects were placed on the median line at $\sim 10 \mathrm{~cm}$ from each wall and the animal was returned to the open field and allowed to explore the objects for $5 \mathrm{~min}$ before being returned to its home cage. After $1 \mathrm{~h}$, one familiar object and one novel object were placed in the open field to the location where the identical objects were placed during the familiarization session and the mouse was allowed to explore them for a 5-min recognition test. The side of the novel object position was randomly assigned so half of the animals were exposed to a novel object placed on the right of the open field and half of the animals were exposed to a novel object placed on the left of the open field.

Between each session, the open field and the objects were carefully cleaned with $70 \%$ ethanol and let dry. Familiarization and recognition sessions were scored for total time spent investigating each object, the number of object interactions and the latency o the first object interaction. Time spend in each side during habituation and familiarization and time spent sniffing two identical objects during the familiarization phase were used to examine an innate side bias. Total time spent sniffing both objects was used as a measure of general exploration.

\section{Marble burying test}

The marble-burying assay is a tool for assessing either anxiety-like and/or repetitive-like behaviors in mice (Thomas et al., 2009). Subjects were tested in a regular clean cage $(28 \mathrm{~cm}$ long $\times 18 \mathrm{~cm}$ wide $\times 12 \mathrm{~cm}$ high) with $3 \mathrm{~cm}$ of fresh bedding. The subject was first placed in the empty cage for a 5-min habituation. It was then temporary placed in an empty clean cage while 20 dark blue glass marbles (15 $\mathrm{mm}$ in diameter) were positioned over the bedding equidistant in a $4 \times 5$ arrangement to cover the whole cage surface. The subject was then returned in the test cage and allowed to explore and bury the marbles during a 15-min session that was videotaped. At the end of the session the subject was removed and the number of marbles buried ( $>50 \%$ marble covered by bedding material) was recorded.

\section{Nest building}

For small rodents, nests are important for heat conservation as well as for reproduction and shelter (Deacon, 2006). Mice were initially single housed in cages containing no environmental enrichment items such as bedding, cardboard houses or tunnels. To test their ability to build nests animals were temporarily single housed. One hour before the dark phase, any building material present in the home cage was removed and replaced by two cotton nestlets (Ancare, NES3600 nestlets). The test was repeated twice and scored on the next morning of the second repeat using the following multicriteria scale adapted from (Deacon, 2006; maximum score $=11$ ): nestlet shredding: $0=$ not at all, $1=$ partially, 2 = fully shredded; nestlet dispersion: $0=$ nestlet dispersed all over the cage, 1 = mostly used to build nest, 2 = fully used to build a nest; nest density: 0: not dense, $1=$ medium density, 2 = high density; nest shape: 0 : no nest, 1 = ball shape, 2 = nest shape but no bottom, $3=$ full nest; presence of walls: $0=$ no walls, $1=$ partial walls, $2=$ nest fully surrounded by walls; maximum score $=11$.

\section{Escape behavior}

Escape behavior evaluated in three different tests all taking place in regular home cages $(28 \mathrm{~cm}$ long $\times 18 \mathrm{~cm}$ wide $\times 12 \mathrm{~cm}$ high) by counting the number of unsuccessful (mouse climbing on cage walls) or successful (mice jumping out of the cage) attempts. The three tests, selected for their increasing anxiogenic properties, were the habituation phase of the buried food test (first test in the home cage set-up, no object at the surface of the bedding), the repetitive novel object contact task (four objects visible at the surface of the bedding) and the marble burying test (20 objects visible at the surface of the bedding). Each test was scored for $10 \mathrm{~min}$

\section{Hyper-reactivity}

Hyper-reactivity was recorded by looking at touch escape response, positional passivity, trunk curl and catalepsy during the handling of the mice using the following scales. Touch escape to cotton-tipped applicator stroke from above starting light and slowly getting firmer recorded over five trials: $0=$ no response, $1=$ mild (escape response to firm stroke), 2 = moderate (rapid response to light stroke), 3 = vigorous (escape response to approach). Positional passivity or struggle response to sequential handling: $0=$ struggles when restrained by tail, $1=$ struggles when restrained by neck (finger grip, not scruffed), 2 = struggles when held supine (on back), $3=$ struggles when restrained by hind legs, $4=$ does not struggle. Trunk curl: $0=$ absent, $1=$ present. Catalepsy when subject front paws are positioned on a rod elevated $3 \mathrm{~cm}$ from floor, the amount of time the animal stayed immobile and kept its paws on rod was recorded, with a maximum cutoff of 120 s over three trials separated by 30 s. Hyper-reactivity was also observed in other tests such as the beam walking tests or the negative geotaxis test.

\section{Stereotypies, repetitive behavior, perseveration}

\section{Repetitive novel object contact task}

This novel object investigation task looks for specific unfamiliar objects preference as well as patterned sequences of sequential investigations of those items (Pearson et al., 2011; Steinbach et al., 2016). Subjects were tested in a regular clean cage $(28 \mathrm{~cm}$ long $\times 18 \mathrm{~cm}$ wide $\times 12 \mathrm{~cm}$ high) with $1 \mathrm{~cm}$ of fresh bedding. The subject was first placed in the empty cage for a 20-min habituation. It was then temporary placed in an empty clean cage while four unfamiliar objects (a Lego piece, $3 \mathrm{~cm}$ in length; a jack, $4 \mathrm{~cm}$ in length; a dice, $1.5 \mathrm{~cm}$ in length; and a 
bowling pin, $3.5 \mathrm{~cm}$ in length) were place in the cage's corners at $\sim 3 \mathrm{~cm}$ from the edges. The subject was then able to investigate the environment and objects during a 10-min session that was videotaped. The videos were manually scored for the occurrence of investigation of each of the four toys. Investigation was defined as clear facial or vibrissae contact with objects or burying of the objects. The number of contacts and the cumulative contact time was evaluated for each object. to determine if there was a genotype effect on the tendency to display preferences for particular toys, the frequencies of contact with each object were ranked in decreasing order from maximum to minimum preference for each subject and the frequencies were averaged by group and compared. To assess the pattern of object investigation, each specific toy was given an arbitrary number (1-4) and all possible three-digit and four-digit combinations without repeat numbers were identified. For both three- and fourobject sequences the total number of choice, the number of unique sequences, and the number of choices of the three most repeated sequence was calculated for each subject as described in (Steinbach et al., 2016). To take in account the overall mouse activity, the percentage of top, top two, and top three preferred choices over the total number of choices were also calculated.

\section{Barnes maze}

The Barnes maze is a test of spatial memory comparable to a dry version of the Morris water maze (Barnes, 1979). In this assay, mice use spatial memory and navigation skills to orient themselves thanks to extra-maze cues placed in the test room, with the goal of locating one of 20 identical holes evenly spaced around the edge of a brightly-lit $100 \mathrm{~cm}$ in diameter circular arena (Maze Engineers). While most of the holes (nontarget) have nothing beneath them and lead nowhere, the target escape hole leads to shelter in a desirably darkened and enclosed goal box below the table. Two days before the beginning of the training, habituation was performed by allowing each subject to freely explore the arena (without escape box) under modest light for $5 \mathrm{~min}$. At the end of the second habituation, subjects were pre-trained to learn of the presence of the escape hole by placing them for $1 \mathrm{~min}$ in a clear box in the middle of the arena under bright light conditions. After $1 \mathrm{~min}$, the box was lifted up and the subject was gently guided near the escape hole selected randomly on the table, allowing it to enter the hole and remain inside for $1 \mathrm{~min}$. For the initial training, animals were trained for $4 \mathrm{~d}$ to locate the escape box (in a position different from the pre-training). All trials began with the subject in a clear box in the center of the table. The trial started when the box was lifted up. If the subject located and entered the escape box within $3 \mathrm{~min}$, it was left in the box for $1 \mathrm{~min}$. If the subject failed to find the escape box within $3 \mathrm{~min}$, it was gently guided to near the escape hole, and allowed to stay in the box for $1 \mathrm{~min}$. Animals received four trials per day with an intertrial interval of 20 min for $4 \mathrm{~d}$. After each trial, the maze and the escape box were cleaned using cleaning wipes to remove odors and fresh bedding was placed in the escape box. On the fifth day, animals were tested for 3 min without the escape box for a probe test.
Time spent in the different quadrants was recorded. For the reversal training, the escape hole was moved to the opposite position on the maze and animals received four additional days of training followed by a reversal probe test on the fifth day. All trials were recorded by overhead camera (Noldus Ethovision) and scored for distance and latency to find escape box.

\section{Cognition}

\section{Y-maze test}

Y-maze alternation is a test of working memory based on the natural tendency of mice to explore new territory whenever possible. Mice were placed in the center of a Y-maze (three 5-cm-wide and 50-cm-long arms, each set $130^{\circ}$ from each other) and given $15 \mathrm{~min}$ to freely explore the three arms of the maze. The number of arm entries and the number of triads were recorded to calculate the percentage of alternation. An entry occurs when all four limbs are within the arm. A successful score is defined by three successive choices that includes one instance of each arm by the total number of opportunities for alternation. A type 1 error is determined by three consecutive choices where the first and third choices are identical. A type 2 error is defined by three consecutive choices where the second and third choices are identical. Perseverance is defined as three or more repetitive entries in the same arm.

\section{Contextual and cued fear conditioning}

To isolate the effects of cued and contextual fear conditioning, a 3-d assay was employed. During the training session, the mice were placed in an ethanol cleaned contextual box with a bar floor, black and white striped walls in which all movements can be recorded (Med Associate fear conditioning boxes coupled with Noldus Ethovision for control an analysis) and given $5 \mathrm{~min}$ to habituate. Movements were then recorded for $540 \mathrm{~s}$. At 120,260 , and $400 \mathrm{~s}$ after the beginning of the recording, the mice were exposed to a 20-s tone (80 dB, $2 \mathrm{kHz})$ and coterminating shock ( $1 \mathrm{~s}, 0.7 \mathrm{~mA})$. Twenty-four hours after the training phase, the animals were tested for contextual memory in the identical enclosure and movements were recorded for $240 \mathrm{~s}$ to assess the ability of the animal to remember the context in which the shocks had occurred the previous day. Forty-eight hours after the training phase, the animals were tested for cued memory in a different context (isopropanol cleaned, white wall insert over a mesh grid floor). They were recorded for $330 \mathrm{~s}$ and were presented with the identical tone from the training session at $120 \mathrm{~s}$, and $260 \mathrm{~s}$ after the beginning of the recording session to assess the ability of each animal to remember the tone and pair it with the shock from training session. The three sessions were recorded using a camera located on the side of the boxes. Freezing, defined as lack of movement except for respiration, was scored using Noldus Ethovision software during each phase.

\section{Anxiety \\ Elevated zero-maze}

Fear and anxiety were tested in an elevated zero-maze. The apparatus consisted of a circular black Plexiglas runway, $5 \mathrm{~cm}$ wide, $60 \mathrm{~cm}$ in diameter, and raised $60 \mathrm{~cm}$ 
off the ground (Maze Engineers). The runway was divided equally into four alternating quadrants of open arcs, enclosed only by a $1 \mathrm{~cm}$ inch lip, and closed arcs, with $25-\mathrm{cm}$ walls. All subjects received one 5-min trial on two consecutive days starting in the center of a closed arm and were recorded by video tracking (Noldus Ethovision). Measures of cumulative open and closed arc times, latency to enter an open arc for the first time (for trials with a closed arc start), total open arm entries, latency to completely cross an open arc for the first time (for trials with a closed arc start) between two closed arcs, closed arc dipping (body in closed arc, head in open arc), open arc dipping (body in open arc, head outside of the maze) were calculated using the mean of the two trials.

\section{Open field}

The vertical activity in the open field was scored by counting the numbers of wall rears (while touching a side of the open field) and free-standing rears. The thigmotaxis was measured by quantifying the amount of time or distance traveled on the side of the open field compared to the center of the open field.

\section{Statistical analyses}

Shank $3^{\Delta 4-22}$ wild-type, heterozygous, and knock-out littermates were compared for each parameter. Statistical analyses were performed with SPSS 23.0 software using different types of ANOVA with or without repeated time measures with genotype as independent variable followed by Tukey pair-wise comparisons and correction for multiple comparisons if needed or equivalent nonparametric tests when required. Newborn developmental milestones were analyzed by two-way ANCOVA using genotype and gender as between-subject factors and litter number as co-variate to take in account possible gender and litter effects. As we did not observe a gender effect, males and females were grouped together in figures and tables. to account for possible cohort effects, cohorts 3 and 4 were analyzed either together using two-way ANOVA with genotype and cohort as betweensubject factors or separately using ANOVA or KruskalWallis tests. Figures represent results for both cohorts analyzed together. Each cohort data and all statistical results including cohort effects are reported in tables and corresponding extended data tables. In tests comparing activity in two or more locations (open field thigmotaxis, social preference test, social transmission of food preference, novel object recognition, zero-maze) genotype $x$ zone interactions were assessed using repeated measures. When sphericity was found violated, the Greenhouse-Geisser values were reported. The distribution of the genotypes was compared to Mendelian expectation using Pearson's $\chi^{2}$ test, the survival curves were analyzed using survival Kaplan-Meyer $\chi^{2}$. The comparison to chance level was evaluated using either one-sample $t$ test or Wilcoxon test. Normality was assessed using data visualization and Shapiro-Wilk test. All values are expressed as mean \pm SEM.

\section{Results}

\section{Generation of a Shank $3^{\Delta 4-22}$ mouse with a complete deletion of the Shank3 gene}

A mouse line with a complete disruption of the Shank3 gene was generated by retargeting ES cells previously used to disrupt exons 4 through 9 (Bozdagi et al., 2010). To do this, an additional loxP site was inserted directly after exon 22 while leaving intact the two existing loxP sites flanking exons 4 and 9 (Fig. 1A). To generate the Shank $3^{\Delta 4-22}$ mouse line used in the present study, the floxed allele was then excised by breeding with a CMVCre transgenic line resulting in a deletion of exons 4-22 and therefore a constitutive disruption of all the Shank3 murine isoforms.

Immunoblot analyses using antibodies which crossreact either with an epitope in the $\mathrm{SH} 3$ domain (antibody N367/62; Fig. 1B, left panel) or with the $\mathrm{COOH}$ terminal (antibody H1160, Fig. 1B, right panel) showed no expression of Shank3 protein in post synaptic density fractions from Shank $3^{\Delta 4-22}$ homozygous mice and reduced expression consistent with haploinsuficiency in the heterozygotes. As in humans, in mice, the Shank3 gene has 22 exons, spans $\sim 58 \mathrm{~kb}$ of genomic DNA, and undergoes complex transcriptional regulation controlled by a combination of five intragenic promoters and extensive alternative splicing resulting in in a complex pattern of mRNA and protein isoforms (Wang et al., 2011, 2014; Kouser et al., 2013; Waga et al., 2014; Speed et al., 2015). The loss of all known major Shank3 mRNA isoforms was confirmed by RT-PCR (Fig. 1C).

The Shank $3^{\Delta 4-22}$ mouse line was maintained on a C57BL/6 background by heterozygote $\times$ heterozygote mating, allowing for the production of all genotypes (wildtype, heterozygous, and homozygous) as littermates. Shank $3^{\Delta 4-22}$ heterozygous and homozygous animals were viable, however abnormal Mendelian ratios were observed at the time of weaning, with a significant deficit for Shank $3^{\Delta 4-22}$ knock-out mice (Fig. 1D; Table 3). Adult survival curves between 1 and 22 months did not show a significant genotype difference with the current sample size, but there was evidence for higher numbers of deaths in Shank $3^{\Delta 4-22}$ homozygous mice between 18 and 22 months (Fig. 1E; Table 3). Although the human clinical SHANK3 mutation is hemizygous, for completeness, we have conducted our studies in Shank3-null mutant mice (homozygous knock-out, $\mathrm{KO}$ ), along with their heterozygous (Het) and wild-type (WT) littermates. The KO mice are instrumental to understand the function of Shank3, while the Het mice have significantly greater construct validity for PMS, a haploinsufficiency syndrome. To ensure the robustness of behavioral abnormalities in the adult mice, two cohorts representing all three genotypes were compared. All the cohorts used in the present study are described in Table 2.

\section{Developmental milestones in Shank $3^{\Delta 4-22}$ neonates}

Ten litters were used to study developmental milestones. The average litter size was 7.2 pups (ranging from five to nine), with 54 surviving passed postnatal day 2 (28 males and 26 females). As very limited gender effects 
Table 3. Genotype distribution at weaning and postnatal mortality

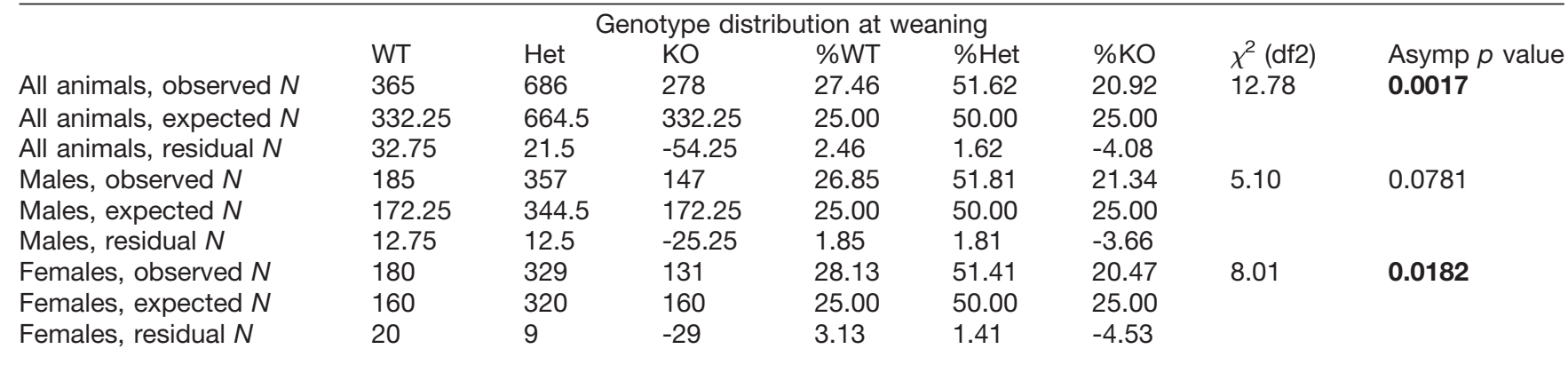

were observed (for detailed analysis, see Table 4), males and females were analyzed together using both genotype and gender as fixed factors and the litter number as a covariate.

Developmental delays were observed in the Shank $3^{\Delta 4-22}$ homozygote neonates in several of the parameters studied (Fig. 2; Extended Data Fig. 2-1; Table 4). While the birth weight was not significantly different, the growth rate of Shank $3^{\Delta 4-22}$ homozygote pups was slower and by P14, the weight of Shank3 $\Delta 4-22$ homozygous mice was significantly lower than the weight of their wild-type littermates (Fig. 2A). Additionally, an unusual postnatal mortality was observed when breeding heterozygous animals together, with $6.9 \%$ of the pups dying between birth and P1. Eighty-six dead pups were genotyped, showing that the percentage of Shank $3^{\Delta 4-22}$ homozygote knock-out mice dying at or shortly after birth was higher than expected if the death was equally affecting all the genotypes (WT: $n=20$, Het $=33$, KO: $n=33, \chi^{2}$ df2 $\left.=8.66, p=0.0137\right)$, this could explain, at least partially, the deficit observed at weaning. No differences were observed in any of the other physical developmental milestones, including eye opening, ear opening, tooth eruption or fur development (Extended Data Fig. 2-1A-D; Table 4).

A significant delay was observed for Shank $3^{\Delta 4-22}$ homozygotes in the response to auditory startle (Fig. 2B) and in the mid-air righting task (Fig. $2 C$ ) although all the mice were able to properly respond at the end of the observation period. In the wire suspension (Fig. 2D) and grasping reflex (Fig. 2E) tasks, however, not only was the acquisition of the response delayed, but Shank $3^{\Delta 4-22}$ homozygous animals remained significantly impaired until the time of weaning. In the negative geotaxis test, an initial delay was observed at P5 were most wild-type animals were able to turn while homozygous and heterozygous Shank $3^{\Delta 4-22}$ animals were still falling or staying in the starting position (Fig. 2F). Moreover, after P9 when most of the animals were able to master the task, higher reactivity (characterized by a shorter latency to turn) was observed for the Shank $3^{\Delta 4-22}$ homozygous mice. The acquisition of the rooting reflex was similar for the three groups; however, a premature disappearance of the reflex was observed in both the Shank $3^{\Delta 4-22}$ heterozygous and homozygous pups (Extended Data Fig. 2-1E; Table 4).

Other sensory-motor and neurologic milestones such as cliff aversion, ear twitch, surface righting, negative geotaxis, and open field crossing (Extended Data Fig. $2-1 F-I$; Table 4) were not significantly affected by the disruption of the Shank3 gene.

Ultrasonic vocalizations were recorded at postnatal day 6 on an independent cohort of mice and a genotype difference was detected in the number and quality of ultrasonic vocalizations emitted by the pups (Table 4). Shank $3^{\Delta 4-22}$ heterozygous and homozygous mice emitted fewer ultrasonic vocalizations than wild-type littermates (Extended Data Fig. 2-1K; Table 4). The total calling time was also affected with Shank $3^{\Delta 4-22}$-deficient mice both spending less time calling and having shorter calls than wild-type littermates. Additionally, the peak amplitude was shorter in Shank3 $3^{\Delta 4-22}$-deficient mice. However, none of these parameters were significantly different, probably due to a high interindividual variability within each group with some animals emitting no vocalizations during the $3-\mathrm{min}$ recording. The percentage of noncallers was higher, although not significantly, in Shank $3^{\Delta 4-22}$-deficient animals. Genotype did not affect the latency to the first call or the peak frequency of calls and no difference was observed in the time course of the emission of ultrasonic vocalizations.

\section{Adult general health in Shank $3^{\Delta 4-22}$-deficient mice}

Adult Shank $3^{\Delta 4-22}$ mice were evaluated for general health at three months of age (Table 5). The three genotypes did not differ on physical measure of weight and length. Additional weight measures at the age of 15 and 20 months showed a trend in reduced weight of Shank $3^{\Delta 4-22}$ homozygous mice compared to their littermates. Genotypes scored similarly and in the normal range for other physical characteristics including coat appearance (grooming, piloerection, patches of missing fur on face or body), skin pigmentation, whisker appearance, wounding, and palpebral closure. Observation in a beaker or after transfer to a housing cage revealed no abnormalities in term of spontaneous general activity, stereotypies (rears, jumps, circling, wild running), transfer arousal, gait, pelvic, and tail elevation.

\section{Motor functions in Shank $3^{\Delta 4-22}$-deficient mice}

Motor functions were examined using several different paradigms (Table 6). Footprint gait analysis showed normal stance and sway but increased stride in Shank $3^{\Delta 4-22}$ homozygous mice compared to wild-type and heterozy- 
Table 4. Detailed results and statistical analyses related to developmental milestones

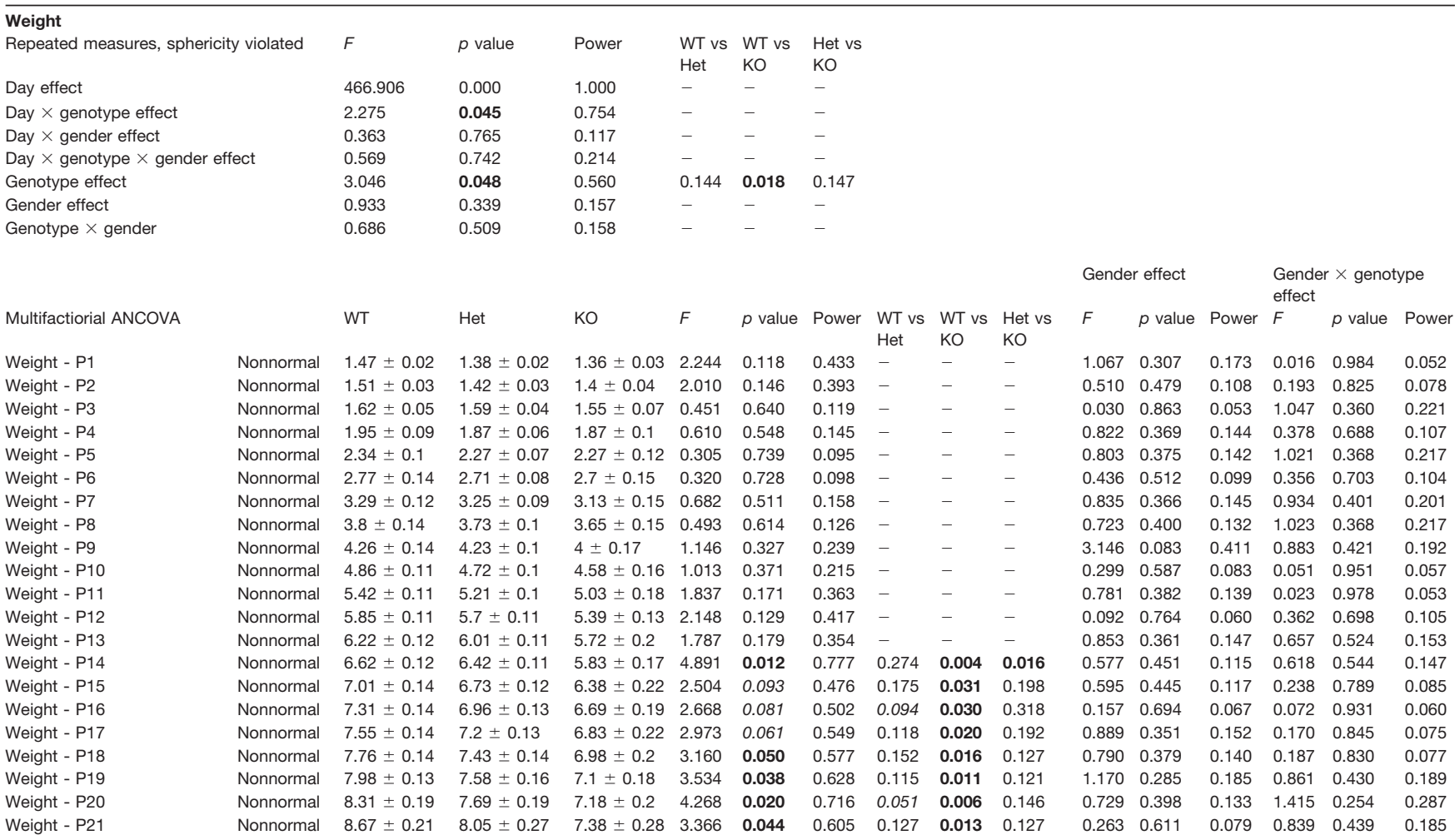

Eye opening

\begin{tabular}{|c|c|c|c|c|c|c|}
\hline Repeated measures, sphericity assumed & $F$ & $p$ value & Power & $\begin{array}{l}\text { WT vs } \\
\text { Het }\end{array}$ & $\begin{array}{l}\text { WT vs } \\
\text { KO }\end{array}$ & $\begin{array}{l}\text { Het vs } \\
\text { KO }\end{array}$ \\
\hline Day effect & 192.080 & 0.000 & 1.000 & - & - & - \\
\hline Day $\times$ genotype effect & 1.565 & 0.190 & 0.469 & - & - & - \\
\hline Day $\times$ gender effect & 0.716 & 0.494 & 0.169 & - & - & - \\
\hline Day $\times$ genotype $\times$ gender effect & 0.653 & 0.629 & 0.544 & & & \\
\hline Genotype effect & 1.403 & 0.257 & 0.285 & - & - & - \\
\hline Gender effect & 1.852 & 0.181 & 0.265 & - & - & - \\
\hline Genotype $\times$ gender & 0.957 & 0.392 & 0.205 & & & \\
\hline
\end{tabular}

\begin{tabular}{|c|c|c|c|c|c|c|c|c|c|c|c|c|c|c|c|c|}
\hline \multirow{2}{*}{\multicolumn{2}{|c|}{ Multifactiorial ANCOVA }} & \multirow[b]{2}{*}{ WT } & \multirow[b]{2}{*}{ Het } & \multirow[b]{2}{*}{$\mathrm{KO}$} & \multirow[b]{2}{*}{$F$} & \multirow[b]{2}{*}{$p$ value } & \multirow[b]{2}{*}{ Power } & \multirow[b]{2}{*}{$\begin{array}{l}\text { WT vs } \\
\text { Het }\end{array}$} & \multirow[b]{2}{*}{$\begin{array}{l}\text { WT vs } \\
\text { KO }\end{array}$} & \multirow[b]{2}{*}{$\begin{array}{l}\text { Het vs } \\
\mathrm{KO}\end{array}$} & \multirow[b]{2}{*}{$F$} & \multirow[b]{2}{*}{$p$ value } & \multirow[b]{2}{*}{ Power } & \multicolumn{2}{|l|}{ effect } & \multirow[b]{2}{*}{ Power } \\
\hline & & & & & & & & & & & & & & $F$ & $p$ value & \\
\hline Eye opening score - P9 & - & $0 \pm 0$ & $0 \pm 0$ & $0 \pm 0$ & - & - & - & - & - & - & - & - & - & - & - & - \\
\hline Eye opening score - P10 & - & $0 \pm 0$ & $0 \pm 0$ & $0 \pm 0$ & - & - & - & - & - & - & - & - & - & - & - & - \\
\hline Eye opening score - P11 & - & $0 \pm 0$ & $0 \pm 0$ & $0 \pm 0$ & - & - & - & - & - & - & - & - & - & - & - & - \\
\hline Eye opening score - P12 & Nonnormal & $0.3 \pm 0.2$ & $0.28 \pm 0.13$ & $0.1 \pm 0.1$ & 0.534 & 0.590 & 0.132 & - & - & - & 1.917 & 0.173 & 0.273 & 0.496 & 0.613 & 0.126 \\
\hline Eye opening score - P13 & Nonnormal & $1.23 \pm 0.34$ & $1.35 \pm 0.25$ & $0.6 \pm 0.3$ & 1.445 & 0.247 & 0.292 & - & - & - & 0.707 & 0.405 & 0.130 & 0.032 & 0.969 & 0.055 \\
\hline Eye opening score - P14 & Nonnormal & $2.38 \pm 0.18$ & $2.75 \pm 0.16$ & $2.1 \pm 0.09$ & 4.723 & 0.014 & 0.761 & 0.134 & 0.167 & 0.005 & 2.464 & 0.124 & 0.336 & 2.248 & 0.118 & 0.433 \\
\hline Eye opening score - P15 & Nonnormal & $3 \pm 0.25$ & $3.1 \pm 0.17$ & $2.7 \pm 0.26$ & 0.646 & 0.529 & 0.151 & - & - & - & 0.043 & 0.837 & 0.055 & 1.262 & 0.293 & 0.260 \\
\hline Eye opening score - P16 & Nonnormal & $4 \pm 0$ & $3.85 \pm 0.06$ & $3.9 \pm 0.1$ & 0.734 & 0.486 & 0.166 & - & - & - & 3.076 & 0.087 & 0.403 & 1.249 & 0.297 & 0.257 \\
\hline Eye opening score - P17 & Nonnormal & $4 \pm 0$ & $3.85 \pm 0.06$ & $4 \pm 0$ & 1.665 & 0.201 & 0.332 & - & - & - & 1.155 & 0.288 & 0.183 & 1.971 & 0.152 & 0.386 \\
\hline Eye opening score - P18 & Nonnormal & $4 \pm 0$ & $3.89 \pm 0.05$ & $4 \pm 0$ & 0.957 & 0.392 & 0.205 & - & - & - & 0.690 & 0.411 & 0.128 & 1.041 & 0.362 & 0.220 \\
\hline Eye opening score - P19 & Nonnormal & $4 \pm 0$ & $3.96 \pm 0.03$ & $4 \pm 0$ & 0.428 & 0.654 & 0.115 & - & - & - & 0.320 & 0.575 & 0.086 & 0.426 & 0.656 & 0.115 \\
\hline Eye opening score - P20 & - & $4 \pm 0$ & $4 \pm 0$ & $4 \pm 0$ & - & - & - & - & - & - & - & - & - & - & - & - \\
\hline Average day of full opening & Nonnormal & $15.53 \pm 0.18$ & $15.57 \pm 0.33$ & $15.9 \pm 0.17$ & 0.469 & 0.629 & 0.122 & - & - & - & 1.472 & 0.232 & 0.220 & 0.749 & 0.479 & 0.169 \\
\hline
\end{tabular}

Ear opening

\begin{tabular}{|c|c|c|c|c|c|c|}
\hline Ear opening & & & & & & \\
\hline Repeated measures, sphericity violated & $F$ & $p$ value & Power & $\begin{array}{l}\text { WT vs } \\
\text { Het }\end{array}$ & $\begin{array}{l}\text { WT vs } \\
\text { KO }\end{array}$ & $\begin{array}{l}\text { Het vs } \\
\mathrm{KO}\end{array}$ \\
\hline Day effect & 316.707 & 0.000 & 1.000 & - & - & - \\
\hline Day $\times$ genotype effect & 0.807 & 0.594 & 0.361 & - & - & - \\
\hline Day $\times$ gender effect & 2.150 & 0.079 & 0.617 & - & - & - \\
\hline Day $\times$ genotype $\times$ gender effect & 1.056 & 0.396 & 0.472 & - & - & - \\
\hline Genotype effect & 0.113 & 0.893 & 0.066 & - & - & - \\
\hline Gender effect & 0.438 & 0.512 & 0.099 & - & - & - \\
\hline Genotype $\times$ gender & 0.676 & 0.514 & 0.156 & - & - & - \\
\hline
\end{tabular}


Table 4. Continued

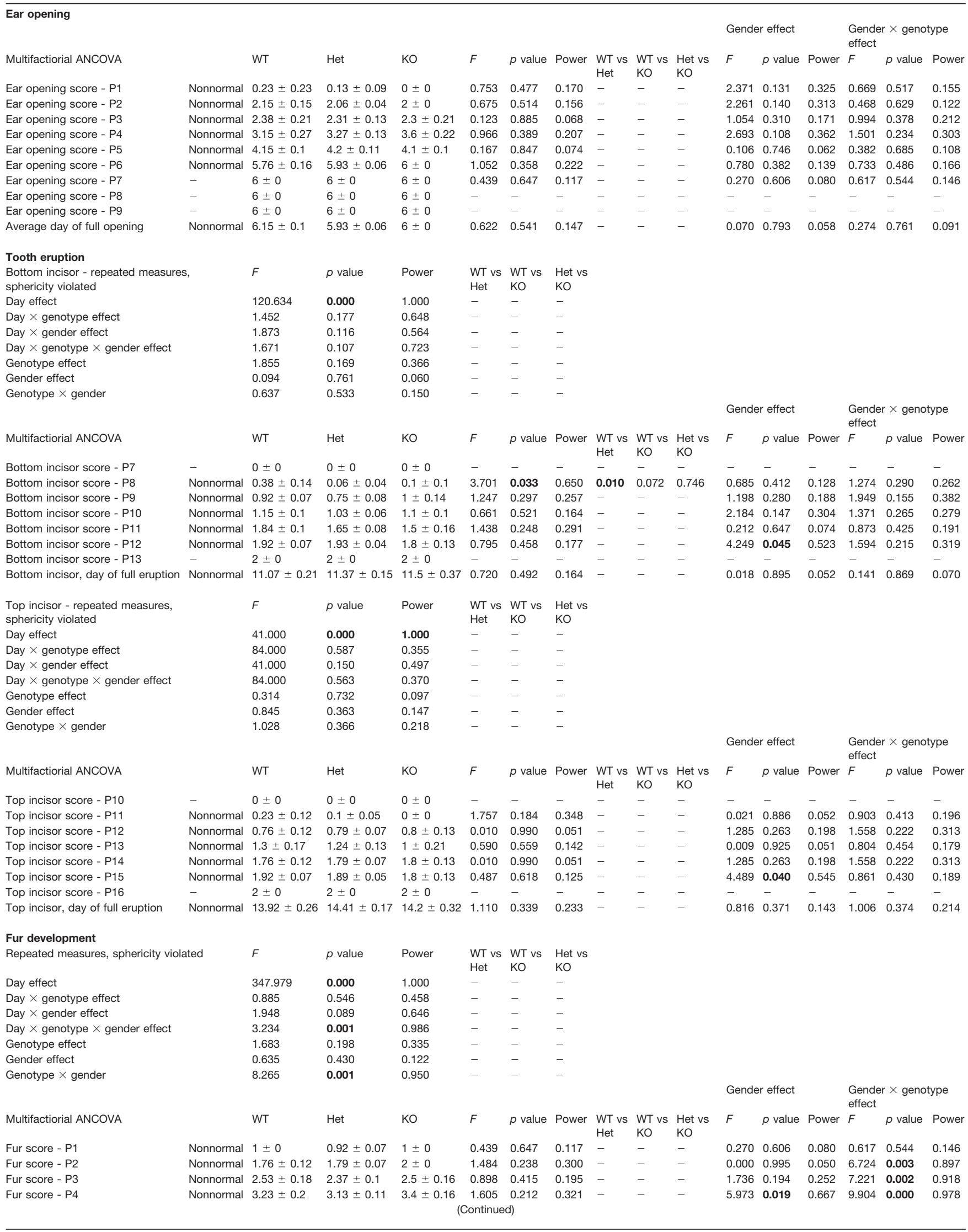


Table 4. Continued

\begin{tabular}{|c|c|c|c|c|c|c|c|c|c|c|c|c|c|c|c|c|}
\hline \multicolumn{17}{|l|}{ Fur development } \\
\hline & & & & & & & & & & \multicolumn{4}{|c|}{ Gender effect } & \multicolumn{3}{|c|}{$\begin{array}{l}\text { Gender } \times \text { genotype } \\
\text { effect }\end{array}$} \\
\hline Multifactiorial ANCOVA & & WT & Het & KO & $F$ & $p$ value & Power & $\begin{array}{c}\text { WT vs } \\
\text { Het }\end{array}$ & $\begin{array}{l}\text { WT vs } \\
\text { KO }\end{array}$ & $\begin{array}{l}\text { Het vs } \\
\text { KO }\end{array}$ & $F$ & $p$ value & Power & $F$ & $p$ value & Power \\
\hline Fur score - P5 & Nonnormal & $3.92 \pm 0.13$ & $3.86 \pm 0.06$ & $4 \pm 0$ & 1.034 & 0.364 & 0.219 & - & - & - & 0.013 & 0.909 & 0.051 & 4.885 & 0.012 & 0.777 \\
\hline Fur score - P6 & Nonnormal & $3.92 \pm 0.13$ & $3.89 \pm 0.05$ & $4 \pm 0$ & 0.657 & 0.523 & 0.153 & - & - & - & 0.090 & 0.766 & 0.060 & 4.026 & 0.025 & 0.689 \\
\hline Fur score - P7 & Nonnormal & $4.15 \pm 0.19$ & $4.27 \pm 0.1$ & $4.5 \pm 0.16$ & 1.002 & 0.375 & 0.213 & - & - & - & 0.353 & 0.556 & 0.089 & 1.378 & 0.263 & 0.281 \\
\hline Fur score - P8 & Nonnormal & $4.69 \pm 0.17$ & $4.62 \pm 0.09$ & $5 \pm 0$ & 2.746 & 0.075 & 0.514 & - & - & - & 1.116 & 0.297 & 0.178 & 4.075 & 0.024 & 0.694 \\
\hline Fur score - P9 & Nonnormal & $5 \pm 0$ & $4.93 \pm 0.04$ & $5 \pm 0$ & 0.927 & 0.403 & 0.200 & - & - & - & 0.604 & 0.441 & 0.118 & 1.203 & 0.310 & 0.249 \\
\hline Fur score - P10 & Nonnormal & $5.23 \pm 0.12$ & $5.24 \pm 0.08$ & $5.3 \pm 0.15$ & 0.125 & 0.882 & 0.068 & - & - & - & 0.007 & 0.936 & 0.051 & 2.343 & 0.108 & 0.450 \\
\hline Fur score - P11 & Nonnormal & $5.53 \pm 0.14$ & $5.72 \pm 0.08$ & $5.8 \pm 0.13$ & 0.906 & 0.411 & 0.196 & - & - & - & 0.997 & 0.324 & 0.164 & 3.754 & 0.031 & 0.656 \\
\hline Fur score - P12 & Nonnormal & $6 \pm 0$ & $5.96 \pm 0.03$ & $6 \pm 0$ & 0.401 & 0.672 & 0.111 & - & - & - & 0.274 & 0.603 & 0.081 & 0.479 & 0.622 & 0.123 \\
\hline Fur score - P13 & - & $6 \pm 0$ & $6 \pm 0$ & $6 \pm 0$ & - & - & . & - & - & - & - & - & - & - & - & - \\
\hline Fur score - P14 & - & $6 \pm 0$ & $6 \pm 0$ & $6 \pm 0$ & - & - & . & - & - & - & - & - & - & - & - & - \\
\hline Day of full fur & Nonnormal & $11.3 \pm 0.2$ & $10.75 \pm 0.37$ & $10.9 \pm 0.23$ & 0.460 & 0.634 & 0.120 & - & - & - & 0.110 & 0.741 & 0.062 & 1.960 & 0.153 & 0.384 \\
\hline
\end{tabular}

Auditory startle

Repeated measures, sphericity violated

Day effect

Day $\times$ genotype effect

Day $\times$ gender effect

Day $\times$ genotype $\times$ gender effect

Genotype effect

Gender effect

Genotype $\times$ gender

$\begin{array}{ll}F & p \text { value } \\ 56.506 & \mathbf{0 . 0 0 0} \\ 3.280 & \mathbf{0 . 0 0 2} \\ 0.283 & 0.873 \\ 1.321 & 0.241 \\ 12.867 & \mathbf{0 . 0 0 0} \\ 0.058 & 0.811 \\ 0.358 & 0.701\end{array}$

Multifactiorial ANCOVA

Percentage of responders - P10 -

WT

Het

KO

$0 \pm 0$

$0 \pm 0$

$0 \pm 0$

Percentage of responders - P11 Nonnormal $15.38+10.41 \quad 13.79 \pm 6.51 \quad 0 \pm 0$

Percentage of responders - P12 Nonnormal $53.84 \pm 14.39 \quad 13.79 \pm 6.51 \quad 0 \pm 0$

Percentage of responders - P13 Nonnormal $53.84 \pm 14.3955 .17 \pm 9.3910 \pm 10$

Percentage of responders - P14 Nonnormal $100 \pm 0$

Percentage of responders - P15 Nonnormal 100

Percentage of responders - P16

Percentage of responders -

$100 \pm 0$
$100 \pm 0$

$86.2 \pm 6.51 \quad 60 \pm 16.32$

$100 \pm 0 \quad 70 \pm 15.27$

WT vs WT vs Het vs

average

First day of two consecutive

Nonnormal $51.92 \pm 1.67$ $100 \pm 0 \quad 100 \pm 0$

Het KO KO

$\begin{array}{lll}- & - & - \\ - & - & -\end{array}$

- $\quad-\quad-$

$\begin{array}{lll}0.070 & 0.000 & 0.000\end{array}$

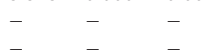

successes

\section{Cliff aversion}

Repeated measures, sphericity violated

$F$
3.957
0.796
0.613
1.266
1.355
0.218
0.116

$p$ value

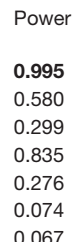

WT vs WT vs Het vs

Day effect

Day $\times$ genotype effect

Day $\times$ gender effect

Day $\times$ genotype $\times$ gender effect

Genotype effect

Gender effect

Genotype $\times$ gender

0.116

Multifactiorial ANCOVA

$$
\begin{aligned}
& 0.000 \\
& 0.702 \\
& 0.782 \\
& 0.209 \\
& 0.269 \\
& 0.643 \\
& 0.891
\end{aligned}
$$

F $\quad p$ value Power WT vs WT vs Het vs

$\begin{array}{llllll} & & & \text { Het } & \text { KO } & \text { KO } \\ - & - & - & - & - & - \\ 1.308 & 0.281 & 0.268 & - & - & -\end{array}$

$\begin{array}{llllll}8.700 & 0.001 & 0.959 & 0.001 & 0.000 & 0.178\end{array}$

$\begin{array}{lllllll}3.045 & 0.058 & 0.560 & 0.969 & 0.043 & 0.023\end{array}$

$\begin{array}{lllllll}3.161 & 0.052 & 0.577 & 0.265 & 0.016 & 0.072\end{array}$

$\begin{array}{lllllll}8.228 & 0.001 & 0.949 & 0.970 & 0.001 & 0.000\end{array}$

$\begin{array}{llllll}- & - & - & - & - & - \\ 7.944 & 0.001 & 0.995 & 0.286 & 0.000 & 0.002\end{array}$

$\begin{array}{llllll}12.867 & 0.000 & 0.941 & 0.070 & 0.000 & 0.000\end{array}$$$
\text { Het KO KO }
$$$$
\begin{array}{lll}
\text { Het } & \text { KO } & \text { KO } \\
- & - & -
\end{array}
$$$$
\begin{array}{lll}
- & - & - \\
- & - & -
\end{array}
$$$$
\begin{array}{lll}
- & - & - \\
- & - & -
\end{array}
$$$$
\begin{array}{lll}
- & - & - \\
- & - & - \\
- & - & -
\end{array}
$$$$
0.067-\quad-\quad-
$$

\begin{tabular}{lllllll}
\multicolumn{2}{l}{ Gender effect } & \multicolumn{5}{c}{$\begin{array}{l}\text { Gender } \times \text { genotype } \\
\text { effect }\end{array}$} \\
$F$ & & $p$ value & Power & $F$ & $p$ value & Power \\
& & & & & & \\
- & - & - & - & - & - \\
0.001 & 0.971 & 0.050 & 3.054 & 0.057 & 0.561 \\
0.488 & 0.488 & 0.105 & 1.584 & 0.217 & 0.318 \\
0.238 & 0.628 & 0.077 & 1.082 & 0.348 & 0.228 \\
0.000 & 0.990 & 0.050 & 0.009 & 0.991 & 0.051 \\
1.019 & 0.318 & 0.167 & 0.865 & 0.428 & 0.189 \\
- & - & - & - & - & - \\
0.466 & 0.498 & 0.056 & 0.144 & 0.866 & 0.104 \\
0.058 & 0.811 & 0.102 & 0.358 & 0.701 & 0.071
\end{tabular} $0.058-0.811$

Gender effect Gender $\times$ genotype effect

WT

$\begin{array}{llllllll}\text { Nonnormal } & 23.61 \pm 2.77 & 24.44 \pm 1.86 & 21.77 \pm 3.56 & 0.745 & 0.481 & 0.168\end{array}$ Nonnormal $15.76 \pm 3.37$ Nonnormal $8.61 \pm 3$

Nonnormal $6.84+2.86$

$\begin{array}{llll} & & \end{array}$

$\begin{array}{llll}\text { Nonnormal } & 10.69 \pm 3.19 & 9.72 \pm 2.18 & 3.8 \pm 0.92\end{array}$

Nonnormal $13.46 \pm 3.45 \quad 7.03 \pm 1.62 \quad 5.6 \pm 2.77$

Nonnormal $9.3+2.66$

Nonnormal $8.61 \pm 1.52$

Nonnormal $5.46 \pm 1.7$

Nonnormal $5.76 \pm 2.16$

$11.51 \pm 2.389 \pm 3.55$

$8.93 \pm 1.67 \quad 5.3 \pm 1.21$

$6.48 \pm 1.42 \quad 5.3 \pm 2.78$

$4.1 \pm 0.67 \quad 4.3 \pm 1.67$

$0.44 \pm 0.11 \quad 0.6 \pm 0.26$

Nonnormal $1.07 \pm 0.53$

Nonnormal $4.84 \pm 0.29$

$4.75 \pm 0.22 \quad 5.2 \pm 0.55$

Nonnormal $4.38 \pm 0.33 \quad 4.06 \pm 0.14 \quad 4.5 \pm 0.45$

Nonnormal $11.25 \pm 1.25 \quad 9.36 \pm 0.65 \quad 8.5 \pm 0.85$
F $\quad p$ value Power WT vs WT vs $\begin{aligned} & \text { Het vs } \\ & \text { Het KO KO }\end{aligned}$

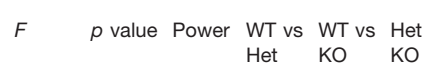

$F \quad p$ value Power $F \quad p$ value Power

$\begin{array}{llllll}0.482 & 0.491 & 0.104 & 0.866 & 0.428 & 0.189\end{array}$

$\begin{array}{llllll}0.056 & 0.814 & 0.056 & 1.513 & 0.232 & 0.304\end{array}$

$\begin{array}{llllll}0.340 & 0.563 & 0.088 & 1.916 & 0.160 & 0.376\end{array}$

$\begin{array}{llllll}0.898 & 0.349 & 0.153 & 2.242 & 0.119 & 0.432\end{array}$

$\begin{array}{llllll}0.040 & 0.843 & 0.054 & 2.223 & 0.121 & 0.429\end{array}$

$\begin{array}{llllll}1.259 & 0.268 & 0.195 & 0.368 & 0.694 & 0.105\end{array}$

$\begin{array}{llllll}0.618 & 0.436 & 0.120 & 0.315 & 0.731 & 0.097\end{array}$

$\begin{array}{lllllll}0.011 & 0.917 & 0.051 & 0.285 & 0.753 & 0.092\end{array}$

$\begin{array}{llllll}1.109 & 0.298 & 0.177 & 0.316 & 0.731 & 0.097\end{array}$

$\begin{array}{llllll}0.263 & 0.611 & 0.079 & 1.486 & 0.238 & 0.300\end{array}$

$\begin{array}{lllllll}0.001 & 0.975 & 0.050 & 0.651 & 0.527 & 0.152\end{array}$

$\begin{array}{llllll}2.134 & 0.151 & 0.298 & 0.791 & 0.460 & 0.176\end{array}$

$\begin{array}{lllllll}1.345 & 0.253 & 0.205 & 0.941 & 0.398 & 0.202\end{array}$

$\begin{array}{llllll}3.688 & 0.061 & 0.467 & 1.125 & 0.334 & 0.235\end{array}$

$\begin{array}{llllll}2.726 & 0.106 & 0.365 & 2.174 & 0.126 & 0.421\end{array}$

$\begin{array}{llllll}0.037 & 0.849 & 0.054 & 1.044 & 0.361 & 0.221\end{array}$

$\begin{array}{llllll}0.259 & 0.613 & 0.079 & 0.119 & 0.888 & 0.067\end{array}$ 
Table 4. Continued

\begin{tabular}{|c|c|c|c|c|c|c|c|c|c|c|c|c|c|c|c|c|}
\hline \multicolumn{17}{|l|}{ Ear twitch reflex } \\
\hline \multicolumn{2}{|l|}{ Repeated measures, sphericity violated } & $F$ & $p$ value & Power & $\begin{array}{l}\text { WT vs } \\
\text { Het }\end{array}$ & $\begin{array}{l}\text { WT vs } \\
\text { KO }\end{array}$ & $\begin{array}{l}\text { Het vs } \\
\text { KO }\end{array}$ & & & & & & & & & \\
\hline \multicolumn{2}{|l|}{ Day effect } & 5.197 & 0.000 & 0.994 & - & - & - & & & & & & & & & \\
\hline \multicolumn{2}{|l|}{ Day $\times$ genotype effect } & 0.866 & 0.581 & 0.502 & - & - & - & & & & & & & & & \\
\hline \multicolumn{2}{|l|}{ Day $\times$ gender effect } & 0.830 & 0.547 & 0.325 & - & - & - & & & & & & & & & \\
\hline \multicolumn{2}{|l|}{ Day $\times$ genotype $\times$ gender effect } & 1.115 & 0.348 & 0.637 & - & - & - & & & & & & & & & \\
\hline \multicolumn{2}{|l|}{ Genotype effect } & 2.147 & 0.129 & 0.416 & - & - & - & & & & & & & & & \\
\hline \multicolumn{2}{|l|}{ Gender effect } & 0.152 & 0.698 & 0.067 & - & - & - & & & & & & & & & \\
\hline \multirow{2}{*}{\multicolumn{2}{|c|}{ Genotype $\times$ gender }} & 0.834 & 0.441 & 0.184 & - & - & - & & & & & & & & & \\
\hline & & & & & & & & & & & \multicolumn{3}{|c|}{ Gender effect } & \multicolumn{3}{|c|}{$\begin{array}{l}\text { Gender } \times \text { genotype } \\
\text { effect }\end{array}$} \\
\hline \multicolumn{2}{|l|}{ Multifactiorial ANCOVA } & WT & Het & $\mathrm{KO}$ & $F$ & $p$ value & Power & $\begin{array}{l}\text { WT vs } \\
\text { Het }\end{array}$ & $\begin{array}{l}\text { WT vs } \\
\text { KO }\end{array}$ & $\begin{array}{l}\text { Het vs } \\
\text { KO }\end{array}$ & $F$ & $p$ value & Power & $F$ & $p$ value & Power \\
\hline Percentage of responders - P7 & Nonnormal & $46.15 \pm 14.39$ & $17.24 \pm 7.13$ & $50 \pm 16.66$ & 2.610 & 0.085 & 0.493 & 0.076 & 0.870 & 0.073 & 0.347 & 0.559 & 0.089 & 0.888 & 0.419 & 0.193 \\
\hline Percentage of responders - P8 & Nonnormal & $30.76 \pm 13.32$ & $6.89 \pm 4.78$ & $30 \pm 15.27$ & 2.340 & 0.108 & 0.449 & - & - & - & 0.089 & 0.766 & 0.060 & 0.834 & 0.441 & 0.184 \\
\hline Percentage of responders - P9 & Nonnormal & $46.15 \pm 14.39$ & $17.24 \pm 7.13$ & $10 \pm 10$ & 2.860 & 0.068 & 0.532 & 0.046 & 0.037 & 0.551 & 0.244 & 0.624 & 0.077 & 0.368 & 0.694 & 0.105 \\
\hline Percentage of responders - P10 & Nonnormal & $38.46 \pm 14.04$ & $24.13 \pm 8.08$ & $30 \pm 15.27$ & 0.394 & 0.677 & 0.110 & - & - & - & 3.054 & 0.088 & 0.401 & 1.829 & 0.173 & 0.361 \\
\hline Percentage of responders - P11 & Nonnormal & $53.84 \pm 14.39$ & $62.06 \pm 9.16$ & $70 \pm 15.27$ & 0.461 & 0.633 & 0.121 & - & - & - & 0.143 & 0.707 & 0.066 & 2.804 & 0.071 & 0.524 \\
\hline Percentage of responders - P12 & Nonnormal & $46.15 \pm 14.39$ & $41.37 \pm 9.3$ & $50 \pm 16.66$ & 0.214 & 0.808 & 0.081 & - & - & - & 0.527 & 0.472 & 0.109 & 0.270 & 0.765 & 0.090 \\
\hline Percentage of responders - P13 & Nonnormal & $46.15 \pm 14.39$ & $48.27 \pm 9.44$ & $60 \pm 16.32$ & 0.064 & 0.938 & 0.059 & - & - & - & 1.507 & 0.226 & 0.225 & 0.890 & 0.418 & 0.194 \\
\hline Percentage of responders - P14 & Nonnormal & $61.53 \pm 14.04$ & $72.41 \pm 8.44$ & $80 \pm 13.33$ & 0.379 & 0.687 & 0.107 & - & - & - & 0.022 & 0.882 & 0.052 & 0.468 & 0.629 & 0.122 \\
\hline Percentage of responders - P15 & Nonnormal & $100 \pm 0$ & $96.55 \pm 3.44$ & $90 \pm 10$ & 0.497 & 0.612 & 0.126 & - & - & - & 0.569 & 0.455 & 0.114 & 2.150 & 0.129 & 0.417 \\
\hline Percentage of responders - Average & Nonnormal & $52.13 \pm 5.39$ & $42.91 \pm 2.62$ & $52.22 \pm 3.33$ & 2.147 & 0.129 & 0.416 & - & - & - & 0.152 & 0.698 & 0.067 & 0.834 & 0.441 & 0.184 \\
\hline First day of two consecutive successes & Nonnormal & $9.23 \pm 0.63$ & $10.13 \pm 0.36$ & $8.8 \pm 0.61$ & 1.851 & 0.169 & 0.365 & - & - & - & 0.137 & 0.713 & 0.065 & 1.106 & 0.340 & 0.232 \\
\hline
\end{tabular}

\section{Rooting reflex}

Repeated measures, sphericity violated

Day effect

Day $\times$ genotype effect

Day $\times$ gender effect

Day $\times$ genotype $\times$ gender effect

Genotype effect

Gender effect

Genotype $\times$ gender

Multifactiorial ANCOVA

Percentage of responders - P2 Percentage of responders - P3 Percentage of responders - $P 4$ Percentage of responders - P5 Percentage of responders - P6 Percentage of responders - P7 Percentage of responders - P8 Percentage of responders - P9 Percentage of responders - P10 Percentage of responders - P11 Percentage of responders - P12 Day of first observation

Day of last observation

$\begin{array}{lll}F & p \text { value } & \text { Power } \\ & & \\ 8.013 & \mathbf{0 . 0 0 0} & 0.999 \\ 1.657 & 0.107 & 0.735 \\ 0.847 & 0.503 & 0.276 \\ 1.347 & 0.219 & 0.625 \\ 1.689 & 0.196 & 0.336 \\ 4.277 & 0.045 & 0.525 \\ 0.283 & 0.755 & 0.092\end{array}$

WT

Het

$\mathrm{KO}$

Nonnormal $23.07 \pm 12.16 \quad 6.89 \pm 4.78 \quad 20 \pm 13.33$ Nonnormal $38.46 \pm 14.0434 .48 \pm 8.98 \quad 20 \pm 13.33$ Nonnormal $46.15 \pm 14.3958 .62 \pm 9.3 \quad 50 \pm 16.66$ Nonnormal $61.53 \pm 14.04 \quad 82.75 \pm 7.13 \quad 60 \pm 16.32$ Nonnormal $92.3 \pm 7.69 \quad 68.96 \pm 8.74 \quad 70 \pm 15.27$ Nonnormal $84.61+10.4168 .96+8.7490 \pm 10$

Nonnormal $84.61 \pm 10.4158 .62 \pm 9.3 \quad 40 \pm 16.32$ Nonnormal $76.92 \pm 12.1631 .03 \pm 8.74 \quad 40 \pm 16.32$ Nonnormal $38.46 \pm 14.0413 .79 \pm 6.5120 \pm 13.33$ Nonnormal $0 \pm 0$ $0 \pm 0$ $0 \pm 0$ $10 \pm 10$

Nonnormal $4.15 \pm 0.45$ $4.31 \pm 0.28-5 \pm 0.66$ $8.58 \pm 0.3 \quad 9.1 \pm 0.48$

$\begin{array}{lll}\text { WT vs } & \text { WT vs } & \text { Het vs } \\ \text { Het } & \text { KO } & \text { KO } \\ - & - & - \\ - & - & - \\ - & - & - \\ - & - & - \\ - & - & - \\ - & - & - \\ - & - & -\end{array}$

Gender effect Gender $\times$ genotype effect

F $\quad p$ value Power WT vs WT vs Het vs Het KO KO $\begin{array}{lllllll}1.878 & 0.177 & 0.268 & 0.771 & 0.469 & 0.173\end{array}$ $\begin{array}{llllll}0.701 & 0.407 & 0.130 & 2.220 & 0.121 & 0.429\end{array}$ $\begin{array}{lllllll}3.072 & 0.087 & 0.403 & 0.308 & 0.736 & 0.096\end{array}$ $\begin{array}{lllllll}3.120 & 0.084 & 0.408 & 0.395 & 0.676 & 0.110\end{array}$ $\begin{array}{lllllll}0.959 & 0.333 & 0.160 & 1.595 & 0.214 & 0.320\end{array}$ $\begin{array}{lllllll}0.618 & 0.436 & 0.120 & 0.193 & 0.826 & 0.078\end{array}$ $\begin{array}{lllllll}2.183 & 0.147 & 0.304 & 0.616 & 0.545 & 0.146\end{array}$ $\begin{array}{llllll}0.010 & 0.919 & 0.051 & 1.945 & 0.155 & 0.381\end{array}$ $\begin{array}{llllll}2.619 & 0.113 & 0.353 & 2.258 & 0.117 & 0.435\end{array}$ $\begin{array}{llllll}1.546 & 0.220 & 0.229 & 0.757 & 0.475 & 0.170\end{array}$ $\begin{array}{llllll}1.428 & 0.239 & 0.215 & 1.200 & 0.311 & 0.249\end{array}$

\section{Grasping reflex}

Repeated measures, sphericity assumed

Day effect

Day $\times$ genotype effect

Day $\times$ gender effect

Day $\times$ genotype $\times$ gender effect

Genotype effect

Gender effect

Genotype $\times$ gender

\section{Multifactiorial ANCOVA}

Grasping score - P5 Grasping score - P6 Grasping score - P7 Grasping score - P8 Grasping score - P9 Grasping score - P10 Grasping score - P11 Grasping score - P12 Grasping score - P13 Grasping score - P14 Grasping score - Average First day of two consecutive successes (score 4)

$\begin{array}{lll}F & p \text { value } & \text { Power } \\ 28.265 & 0.000 & 1.000 \\ 1.038 & 0.415 & 0.591 \\ 0.534 & 0.850 & 0.208 \\ 1.356 & 0.150 & 0.725 \\ 3.923 & 0.027 & 0.677 \\ 0.052 & 0.821 & 0.056 \\ 0.320 & 0.728 & 0.098\end{array}$

WT Het

Nonnormal $1.3 \pm 0.23$ Nonnormal $2.46 \pm 0.36$ Nonnormal $2.92 \pm 0.28$ Nonnormal $3.38 \pm 0.28$ Nonnormal $3.76 \pm 0.3$ Nonnormal $4.38+0.24$ Nonnormal $5.3 \pm 0.23$ Nonnormal $5.3 \pm 0.23$ Nonnormal $5.69 \pm 0.17$ Nonnormal $5.69 \pm 0.13$ Nonnormal $4.02 \pm 0.13$ Nonnormal $9.38 \pm 0.43$

Het $\quad \mathrm{KO}$

$1.34 \pm 0.16 \quad 1.6 \pm 0.16$ $2.93+0.21 \quad 2.8+0.38$ $3.34 \pm 0.17 \quad 2.9 \pm 0.23$ $3.41 \pm 0.15 \quad 3.2 \pm 0.24$ $3.79 \pm 0.09 \quad 3.8 \pm 0.41$ $4.65+0.19 \quad 3.9+0.37$ $5.41 \pm 0.15 \quad 4.9 \pm 0.31$ $5.37 \pm 0.15 \quad 5 \pm 0.29$ $5.86+0.09 \quad 5.2 \pm 0.24$ $5.68 \pm 0.12 \quad 4.6 \pm 0.26$ $\begin{array}{llllllll} & 3.923 & 0.027 & 0.677 & 0.304 & 0.116 & 0.008\end{array}$ $\begin{array}{lll}\text { WT vs } & \text { WT vs } & \text { Het vs } \\ \text { Het } & \text { KO } & \text { KO } \\ - & - & - \\ - & - & - \\ - & - & - \\ - & - & - \\ 0.304 & 0.116 & 0.008 \\ - & - & - \\ - & - & -\end{array}$ F $\quad p$ value Power WT vs WT vs Het vs Het KO KO

$\begin{array}{llllll}0.197 & 0.822 & 0.079 & - & - & - \\ 0.580 & 0.564 & 0.140 & - & - & - \\ 2.260 & 0.116 & 0.436 & - & - & - \\ 0.316 & 0.731 & 0.097 & - & - & - \\ 0.035 & 0.966 & 0.055 & - & - & - \\ 2.102 & 0.134 & 0.409 & - & - & - \\ 1.591 & 0.215 & 0.319 & - & - & - \\ 0.477 & 0.624 & 0.123 & - & - & - \\ 3.789 & \mathbf{0 . 0 3 0} & 0.660 & 0.399 & 0.092 & \mathbf{0 . 0 0 9} \\ 10.311 & \mathbf{0 . 0 0 0} & 0.982 & 0.945 & \mathbf{0 . 0 0 0} & \mathbf{0 . 0 0 0} \\ 3.923 & \mathbf{0 . 0 2 7} & 0.677 & 0.304 & 0.116 & \mathbf{0 . 0 0 8} \\ 10.068 & \mathbf{0 . 0 0 0} & 0.979 & 0.233 & \mathbf{0 . 0 0 5} & \mathbf{0 . 0 0 0}\end{array}$
Gender $\times$ genotype effect $\begin{array}{llllll}0.877 & 0.354 & 0.150 & 2.268 & 0.115 & 0.437 \\ 0.184 & 0.670 & 0.070 & 0.595 & 0.556 & 0.143 \\ 0.858 & 0.359 & 0.148 & 1.670 & 0.200 & 0.333 \\ 1.283 & 0.264 & 0.198 & 1.183 & 0.316 & 0.246 \\ 0.336 & 0.565 & 0.088 & 1.031 & 0.365 & 0.218 \\ 0.035 & 0.852 & 0.054 & 1.782 & 0.180 & 0.353 \\ 0.016 & 0.899 & 0.052 & 1.314 & 0.279 & 0.269 \\ 0.028 & 0.868 & 0.053 & 0.393 & 0.678 & 0.109 \\ 1.328 & 0.255 & 0.204 & 0.287 & 0.752 & 0.093 \\ 0.034 & 0.855 & 0.054 & 0.334 & 0.718 & 0.100 \\ 0.052 & 0.821 & 0.056 & 0.320 & 0.728 & 0.098 \\ 0.131 & 0.719 & 0.065 & 1.318 & 0.278 & 0.270\end{array}$ 
Table 4. Continued

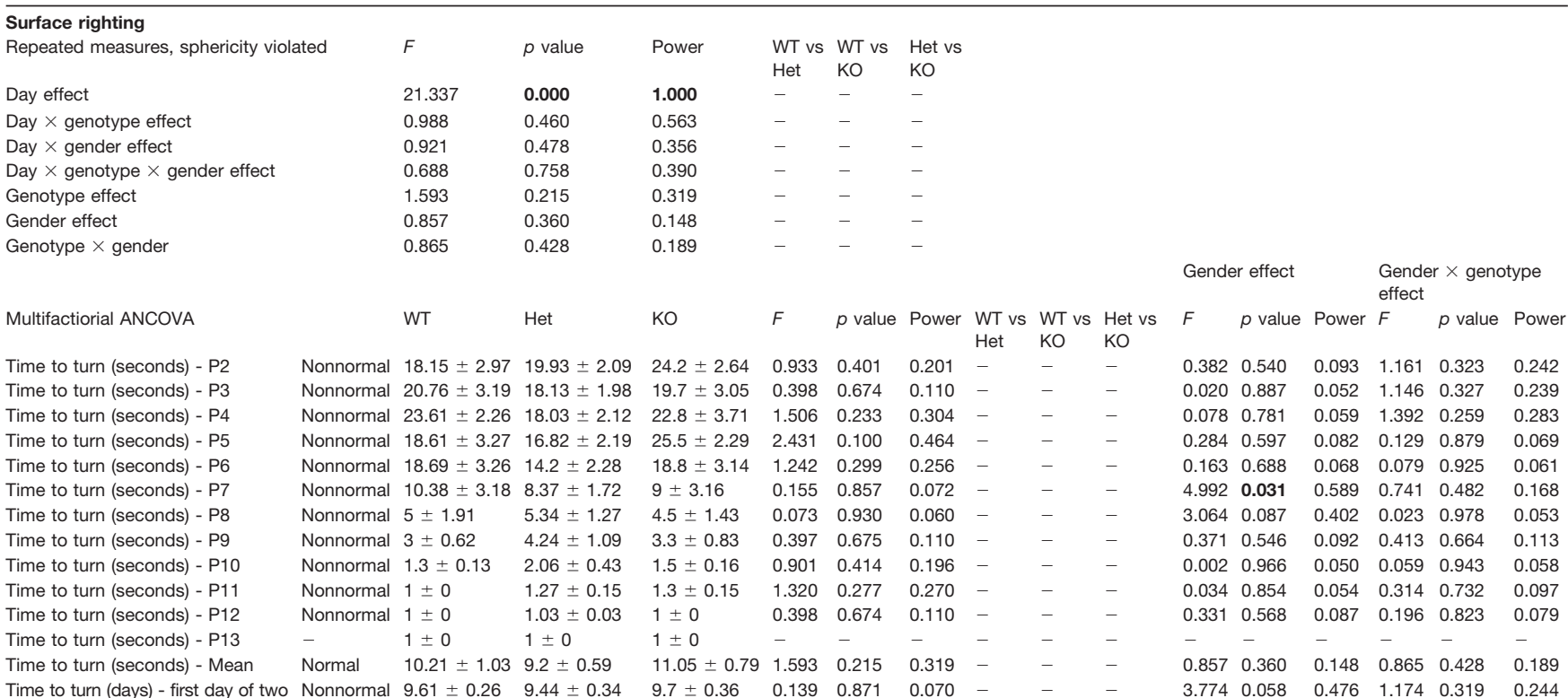

consecutive successe

Negative geotaxis

\begin{tabular}{|c|c|c|c|c|c|c|}
\hline Repeated measures, sphericity violated & $F$ & $p$ value & Power & $\begin{array}{l}\text { WT vs } \\
\text { Het }\end{array}$ & $\begin{array}{l}\text { WT vs } \\
\text { KO }\end{array}$ & $\begin{array}{l}\text { Het vs } \\
\text { KO }\end{array}$ \\
\hline Day effect & 12.128 & 0.000 & 1.000 & - & - & - \\
\hline Day $\times$ genotype effect & 1.526 & 0.086 & 0.895 & - & - & - \\
\hline Day $\times$ gender effect & 1.036 & 0.409 & 0.488 & - & - & - \\
\hline Day $\times$ genotype $\times$ gender effect & 1.386 & 0.144 & 0.855 & - & - & - \\
\hline Genotype effect & 2.110 & 0.133 & 0.410 & - & - & - \\
\hline Gender effect & 0.493 & 0.486 & 0.106 & - & - & - \\
\hline Genotype $\times$ gender & 0.090 & 0.914 & 0.063 & - & - & - \\
\hline
\end{tabular}

Genotype $\times$ gender

WT Het $\quad$ KO

KO
Time to turn (seconds) - P2
Time to turn (seconds) - P3
Time to turn (seconds) - P4
Time to turn (seconds) - P5
Time to turn (seconds) - P6
Time to turn (seconds) - P7
Time to turn (seconds) - P8
Time to turn (seconds) - P9
Time to turn (seconds) - P10
Time to turn (seconds) - P11
Time to turn (seconds) - P12
Time to turn (seconds) - P13
Time to turn (seconds) - P14
Time to turn (seconds) - Mean
Falls

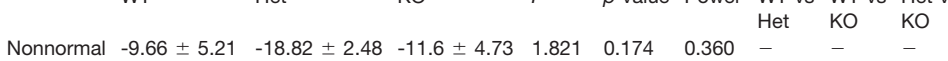

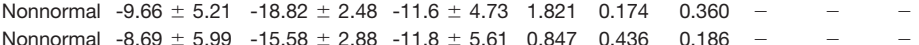

$\begin{array}{llllllllll}\text { Nonnormal } & -6 \pm 5.23 & -10.82 \pm 1.86 & -15.2 \pm 3.51 & 1.537 & 0.226 & 0.309 & - & - & -\end{array}$

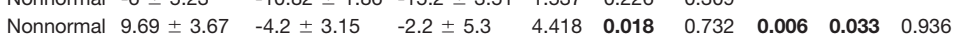

$\begin{array}{lllllll}\text { Nonnormal } & 6.84 \pm 4.38 & 3.2 \pm 2.97 & 8.5 \pm 3.87 & 0.517 & 0.600 & 0.130\end{array}$

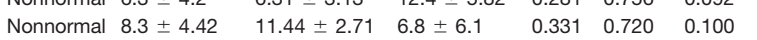

$\begin{array}{llllllllll}\text { Nonnormal } & 12.76 \pm 3.75 & 12.17 \pm 2.44 & 23.1 \pm 1.6 & 3.787 & \mathbf{0 . 0 3 0} & 0.660 & 0.828 & \mathbf{0 . 0 3 5} & \mathbf{0 . 0 1 0}\end{array}$

$\begin{array}{llllllllll}\text { Nonnormal } & 9.84 \pm 2.94 & 10.03 \pm 2.29 & 19 \pm 3.22 & 2.707 & 0.078 & 0.508 & 0.959 & 0.055 & \mathbf{0 . 0 3 2}\end{array}$

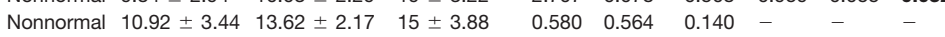

$\begin{array}{llllllllll}\text { Nonnormal } & 11.92 \pm 2.92 & 15.96 \pm 1.74 & 22.2 \pm 1.33 & 3.269 & 0.047 & 0.592 & 0.379 & 0.014 & 0.073\end{array}$

$\begin{array}{llllllllll}\text { Nonnormal } & 15.23 \pm 3.04 & 18.58 \pm 1.5 & 24.1 \pm 0.62 & 3.112 & 0.054 & 0.570 & 0.219 & 0.017 & 0.091\end{array}$

Nonnormal $22.69 \pm 1.43 \quad 21.82 \pm 1.17 \quad 22.3 \pm 2.25 \quad 0.135 \quad 0.874 \quad 0.070-$

$\begin{array}{lllllll}\text { Normal } & 6.99 \pm 1.25 & 4.9 \pm 1.06 & 8.66 \pm 1.1 & 2.110 & 0.133 & 0.410\end{array}$

$\begin{array}{llllll}\text { Nonnormal } 9 \pm 0.83 & 10.31 \pm 0.48 & 9.1 \pm 0.45 & 1.527 & 0.228 & 0.307\end{array}$
Gender effect Gender $\times$ genotype effect

$F \quad p$ value Power $F \quad p$ value Power $\begin{array}{llllll}3.882 & 0.055 & 0.487 & 1.077 & 0.350 & 0.227\end{array}$ $\begin{array}{llllll}0.443 & 0.509 & 0.100 & 0.364 & 0.697 & 0.105\end{array}$ $\begin{array}{llllll}0.656 & 0.422 & 0.124 & 4.540 & 0.016 & 0.744\end{array}$ $\begin{array}{lllllll}0.399 & 0.531 & 0.095 & 0.766 & 0.471 & 0.172\end{array}$ $\begin{array}{lllllll}0.010 & 0.920 & 0.051 & 0.905 & 0.412 & 0.196\end{array}$ $\begin{array}{llllll}0.699 & 0.408 & 0.129 & 0.013 & 0.987 & 0.052\end{array}$ $\begin{array}{llllll}0.124 & 0.726 & 0.064 & 0.862 & 0.429 & 0.189\end{array}$ $\begin{array}{llllll}0.023 & 0.880 & 0.053 & 1.215 & 0.307 & 0.251\end{array}$ $\begin{array}{llllll}2.235 & 0.142 & 0.310 & 0.497 & 0.612 & 0.126\end{array}$ $\begin{array}{llllll}2.444 & 0.125 & 0.334 & 4.258 & 0.020 & 0.715\end{array}$ $\begin{array}{lllllll}0.205 & 0.653 & 0.073 & 2.337 & 0.109 & 0.448\end{array}$ $\begin{array}{llllll}1.431 & 0.238 & 0.216 & 1.112 & 0.338 & 0.233\end{array}$ $\begin{array}{llllll}2.267 & 0.139 & 0.313 & 1.471 & 0.241 & 0.297\end{array}$ $\begin{array}{lllllll}0.493 & 0.486 & 0.106 & 0.090 & 0.914 & 0.063\end{array}$ $\begin{array}{llllll}0.192 & 0.664 & 0.071 & 0.307 & 0.737 & 0.096\end{array}$

\section{Air righting}

Repeated measures, sphericity violated

$\begin{array}{lll}F & p \text { value } & \text { Power } \\ 21.651 & \mathbf{0 . 0 0 0} & 1.000 \\ 3.211 & \mathbf{0 . 0 0 1} & 0.986 \\ 2.423 & 0.037 & 0.760 \\ 1.309 & 0.227 & 0.664 \\ 3.166 & 0.052 & 0.577 \\ 0.464 & 0.499 & 0.102 \\ 1.482 & 0.238 & 0.299\end{array}$

WT Het $\quad$ KO

Nonnormal $0.61 \pm 0.26 \quad 0.41 \pm 0.13$ Nonnormal $0.38 \pm 0.21 \quad 0.93 \pm 0.17$

Nonnormal $1.69 \pm 0.2 \quad 1.44 \pm 0.13$

Nonnormal $1.3 \pm 0.26 \quad 1.44 \pm 0.16$

Nonnormal $1.84 \pm 0.15 \quad 1.89 \pm 0.05$

Nonnormal $1.76 \pm 0.16 \quad 1.68 \pm 0.13$

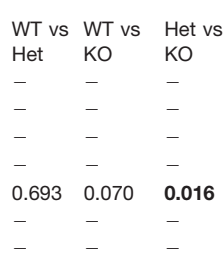

Gender effect

Gender $\times$ genotype effect

F $\quad p$ value Power WT vs WT vs Het vs

$\begin{array}{llllll}1.160 & 0.323 & 0.242 & - & - & -\end{array}$

$0.4 \pm 0.22$

$0.7 \pm 0.3$

$0.4 \pm 0.26$

$1.4 \pm 0.22$

$1.7 \pm 0.21$ $\begin{array}{llll}2.272 & 0.115 & 0.438 & -\end{array}$

$\begin{array}{llllll}5.755 & 0.006 & 0.844 & 0.426 & 0.003 & 0.005\end{array}$

$\begin{array}{llllll}5.407 & 0.008 & 0.819 & 0.703 & 0.015 & \mathbf{0 . 0 0 2}\end{array}$

$\begin{array}{llllll}4.066 & \mathbf{0 . 0 2 4} & 0.693 & 0.742 & \mathbf{0 . 0 3 4} & \mathbf{0 . 0 0 7}\end{array}$

$\begin{array}{llll}0.069 & 0.934 & 0.060 & -\end{array}$
$F \quad p$ value Power $F \quad p$ value Power

$\begin{array}{llllll}5.791 & \mathbf{0 . 0 2 0} & 0.653 & 5.562 & 0.007 & 0.831\end{array}$ $\begin{array}{lllllll}0.004 & 0.948 & 0.050 & 0.439 & 0.648 & 0.117\end{array}$ $\begin{array}{llllll}1.456 & 0.234 & 0.219 & 0.438 & 0.648 & 0.117\end{array}$ $\begin{array}{lllllll}2.641 & 0.111 & 0.356 & 0.639 & 0.533 & 0.150\end{array}$ $\begin{array}{lllllll}0.332 & 0.567 & 0.087 & 0.647 & 0.528 & 0.152\end{array}$ $\begin{array}{llllll}2.104 & 0.154 & 0.295 & 0.396 & 0.675 & 0.110\end{array}$ (Continued) 
Table 4. Continued

\begin{tabular}{|c|c|c|c|c|c|c|c|c|c|c|c|c|c|c|c|c|}
\hline \multicolumn{17}{|l|}{ Air righting } \\
\hline \multicolumn{2}{|c|}{ Repeated measures, sphericity violated } & $F$ & $p$ value & Power & $\begin{array}{l}\text { WT vs } \\
\text { Het }\end{array}$ & $\begin{array}{l}\text { WT vs } \\
\text { KO }\end{array}$ & $\begin{array}{l}\text { Het vs } \\
\text { KO }\end{array}$ & & & & & & & & & \\
\hline Air righting score - P14 & Nonnormal & $2 \pm 0$ & $2 \pm 0$ & $2 \pm 0$ & - & - & . & - & - & - & - & - & . & - & - & ${ }^{\circ}$ \\
\hline Air righting score - P15 & Nonnormal & $1.92 \pm 0.07$ & $2 \pm 0$ & $2 \pm 0$ & 1.621 & 0.209 & 0.324 & - & - & - & 1.716 & 0.197 & 0.249 & 1.464 & 0.242 & 0.296 \\
\hline Air righting score - P16 & Nonnormal & $2 \pm 0$ & $2 \pm 0$ & $2 \pm 0$ & - & - & . & - & - & - & - & - & . & & & 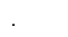 \\
\hline Air righting score - P17 & Nonnormal & $2 \pm 0$ & $1.96 \pm 0.03$ & $2 \pm 0$ & 0.398 & 0.674 & 0.110 & - & - & - & 0.331 & 0.568 & 0.087 & 0.196 & 0.823 & 0.079 \\
\hline Air righting score - P18 & Nonnormal & $2 \pm 0$ & $2 \pm 0$ & $2 \pm 0$ & - & - & . & - & - & - & - & - & . & - & - & . \\
\hline Air righting score - P19 & Nonnormal & $2 \pm 0$ & $2 \pm 0$ & $2 \pm 0$ & - & - & . & - & - & - & - & - & . & - & - & . \\
\hline Air righting score - P20 & Nonnormal & $2 \pm 0$ & $2 \pm 0$ & $2 \pm 0$ & - & - & . & - & - & - & - & - & . & - & - & . \\
\hline Air righting score - Mean & Nonnormal & $1.65 \pm 0.06$ & $1.67 \pm 0.03$ & $1.49 \pm 0.06$ & 3.166 & 0.049 & 0.577 & 0.693 & 0.070 & 0.016 & 0.464 & 0.499 & 0.102 & 1.482 & 0.238 & 0.299 \\
\hline $\begin{array}{l}\text { First day of two consecutive } \\
\text { successes }\end{array}$ & Nonnormal & $11.84 \pm 0.5$ & $11.37 \pm 0.29$ & $12.9 \pm 0.56$ & 2.814 & 0.071 & 0.525 & 0.378 & 0.184 & 0.023 & 0.959 & 0.333 & 0.160 & 1.173 & 0.319 & 0.244 \\
\hline \multicolumn{17}{|l|}{ Wire suspension } \\
\hline \multicolumn{2}{|l|}{ Repeated measures, sphericity violated } & $F$ & $p$ value & Power & $\begin{array}{l}\text { WT vs } \\
\text { Het }\end{array}$ & $\begin{array}{l}\text { WT vs } \\
\text { KO }\end{array}$ & $\begin{array}{l}\text { Het vs } \\
\text { KO }\end{array}$ & & & & & & & & & \\
\hline Day effect & & 16.511 & 0.000 & 1.000 & - & - & - & & & & & & & & & \\
\hline Day $\times$ genotype effect & & 3.538 & 0.000 & 0.994 & - & - & - & & & & & & & & & \\
\hline Day $\times$ gender effect & & 0.497 & 0.782 & 0.186 & - & - & - & & & & & & & & & \\
\hline Day $\times$ genotype $\times$ gender effect & & 0.635 & 0.787 & 0.333 & - & - & - & & & & & & & & & \\
\hline Genotype effect & & 13.553 & 0.000 & 0.997 & 0.013 & 0.000 & 0.001 & & & & & & & & & \\
\hline Gender effect & & 0.303 & 0.585 & 0.084 & - & - & - & & & & & & & & & \\
\hline \multirow[t]{2}{*}{ Genotype $\times$ gender } & & 2.871 & 0.067 & 0.534 & - & - & - & & & & & & & & & \\
\hline & & & & & & & & & & & \multicolumn{3}{|c|}{ Gender effect } & \multicolumn{3}{|c|}{$\begin{array}{l}\text { Gender } \times \text { genotype } \\
\text { effect }\end{array}$} \\
\hline Multifactiorial ANCOVA & & WT & Het & KO & $F$ & $p$ value & Power & $\begin{array}{l}\text { WT vs } \\
\text { Het }\end{array}$ & $\begin{array}{l}\text { WT vs } \\
\text { KO }\end{array}$ & $\begin{array}{l}\text { Het vs } \\
\mathrm{KO}\end{array}$ & $F$ & $p$ value & Power & $F$ & $p$ value & Power \\
\hline Suspension time (seconds) - P11 & Nonnormal & $5.15 \pm 1.44$ & $4.37 \pm 0.63$ & $2.7 \pm 0.47$ & 1.701 & 0.194 & 0.338 & - & - & - & 0.045 & 0.833 & 0.055 & 0.287 & 0.752 & 0.093 \\
\hline Suspension time (seconds) - P12 & Nonnormal & $3.23 \pm 1.06$ & $3.13 \pm 0.45$ & $3.9 \pm 1.65$ & 0.220 & 0.803 & 0.082 & - & - & - & 0.856 & 0.360 & 0.148 & 0.662 & 0.521 & 0.154 \\
\hline Suspension time (seconds) - P13 & Nonnormal & $2.69 \pm 0.47$ & $4 \pm 0.52$ & $2.8 \pm 0.87$ & 1.660 & 0.202 & 0.331 & - & - & - & 2.518 & 0.120 & 0.342 & 0.036 & 0.965 & 0.055 \\
\hline Suspension time (seconds) - P14 & Nonnormal & $7.61 \pm 1.97$ & $5.17 \pm 0.58$ & $3.7 \pm 1.12$ & 2.196 & 0.123 & 0.425 & - & - & - & 0.006 & 0.938 & 0.051 & 1.893 & 0.163 & 0.372 \\
\hline Suspension time (seconds) - P15 & Nonnormal & $9.92 \pm 2.29$ & $4.82 \pm 0.53$ & $1.7 \pm 0.42$ & 10.137 & 0.000 & 0.980 & 0.002 & 0.000 & 0.054 & 0.290 & 0.593 & 0.082 & 0.611 & 0.547 & 0.146 \\
\hline Suspension time (seconds) - P16 & Nonnormal & $13.38 \pm 2.18$ & $6.41 \pm 0.53$ & $3.7 \pm 0.91$ & 15.666 & 0.000 & 0.999 & 0.000 & 0.000 & 0.100 & 0.744 & 0.393 & 0.135 & 1.971 & 0.151 & 0.386 \\
\hline Suspension time (seconds) - P17 & Nonnormal & $18.53 \pm 2.34$ & $11.82 \pm 1.22$ & $9.3 \pm 1.6$ & 6.683 & 0.003 & 0.896 & 0.004 & 0.002 & 0.288 & 0.538 & 0.467 & 0.111 & 1.214 & 0.307 & 0.251 \\
\hline Suspension time (seconds) - P18 & Nonnormal & $16.15 \pm 2.22$ & $18.34 \pm 1.78$ & $11.1 \pm 2.37$ & 2.398 & 0.103 & 0.459 & - & - & - & 0.551 & 0.462 & 0.112 & 0.722 & 0.491 & 0.164 \\
\hline Suspension time (seconds) - P19 & Nonnormal & $18.38 \pm 2.11$ & $19.48 \pm 1.73$ & $8 \pm 1.97$ & 7.474 & 0.002 & 0.927 & 0.921 & 0.003 & 0.001 & 0.000 & 0.995 & 0.050 & 1.609 & 0.212 & 0.322 \\
\hline Suspension time (seconds) - P20 & Nonnormal & $17.07 \pm 2.31$ & $12.13 \pm 1.54$ & $5.6 \pm 0.85$ & 6.858 & 0.003 & 0.903 & 0.053 & 0.001 & 0.019 & 0.646 & 0.426 & 0.123 & 1.283 & 0.287 & 0.264 \\
\hline $\begin{array}{l}\text { Suspension time (seconds) - } \\
\text { Average }\end{array}$ & Nonnormal & $11.21 \pm 1$ & $8.97 \pm 0.55$ & $5.25 \pm 0.54$ & 13.553 & 0.000 & 0.997 & 0.013 & 0.000 & 0.001 & 0.303 & 0.585 & 0.084 & 2.871 & 0.067 & 0.534 \\
\hline Suspension time (seconds) - & Nonnormal & $24.3 \pm 2.06$ & $23.31 \pm 1.37$ & $13.7 \pm 1.99$ & 7.828 & 0.001 & 0.938 & 0.525 & 0.001 & 0.001 & 0.168 & 0.684 & 0.069 & 1.205 & 0.309 & 0.250 \\
\hline
\end{tabular}
Best score

Open field

Repeated measures, sphericity violated

$\begin{array}{lll}F & p \text { value } & \text { Power } \\ 31.056 & \mathbf{0 . 0 0 0} & 1.000 \\ 0.874 & 0.572 & 0.501 \\ 0.630 & 0.702 & 0.247 \\ 1.857 & \mathbf{0 . 0 4 2} & 0.887 \\ 0.117 & 0.890 & 0.067 \\ 0.046 & 0.831 & 0.055 \\ 1.755 & 0.185 & 0.348\end{array}$

$\begin{array}{lll}\text { WT vs } & \text { WT vs } & \text { Het vs } \\ \text { Het } & \text { KO } & \text { KO } \\ - & - & - \\ - & - & - \\ - & - & - \\ - & - & - \\ - & - & - \\ - & - & - \\ - & - & -\end{array}$

Day effect

Day $\times$ genotype effect

Day $\times$ gender effect

Day $\times$ genotype $\times$ gender effect

Genotype effect

Gender effect

Genotype $\times$ gender

1.755

0.185

WT Het

KO

Multifactiorial ANCOVA

Time to escape (seconds) - P8 Time to escape (seconds) - P9 Time to escape (seconds) - P10 Time to escape (seconds) - P11 Time to escape (seconds) - P12 Time to escape (seconds) - P13 Time to escape (seconds) - P14 Time to escape (seconds) - P15 Time to escape (seconds) - P16 Time to escape (seconds) - P17 Time to escape (seconds) - P18 Time to escape (seconds) - P19 Time to escape (seconds) - P20 Time to escape (seconds) - averag First day of two consecutive successes (30-s cutoff)

Utrasonic vocalizations

Number of calls - repeated measures, sphericity assumed

Day effect

Day $\times$ genotype effect

Day $\times$ gender effect

Day $\times$ genotype $\times$ gender effect
Nonnormal $26.07+2.0927 .55+1.1928 .9+0.99 \quad 0.473 \quad 0.626 \quad 0.122$ Het KO KO $\begin{array}{llllll}\text { Nonnormal } 23.3 \pm 2.82 & 21.34 \pm 1.83 & 24.2 \pm 2.64 & 0.305 & 0.739 & 0.095\end{array}$ Nonnormal $20.46 \pm 2.55 \quad 19.96 \pm 1.72 \quad 22.1 \pm 2.34 \quad 0.286 \quad 0.753 \quad 0.093$ Nonnormal $22.07 \pm 2.18 \quad 17.55 \pm 1.9420 .2+3.04 \quad 0.938 \quad 0.399 \quad 0.202$

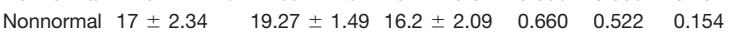

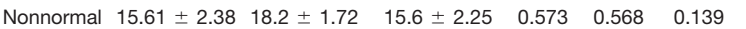
Nonnormal $17.23+2.78 \quad 12.72 \pm 1.6 \quad 10.6+1.14 \quad 2.349 \quad 0.107 \quad 0.450$ Nonnormal $4.92 \pm 0.38 \quad 5.65 \pm 0.39 \quad 7 \pm 1.46 \quad 1.768 \quad 0.183 \quad 0.350$ $\begin{array}{lllllll}\text { Nonnormal } & 3.61 \pm 0.28 & 3.65 \pm 0.25 & 4.5 \pm 0.87 & 1.119 & 0.336 & 0.234\end{array}$ $\begin{array}{llllllllll}\text { Nonnormal } & 2.46 \pm 0.24 & 3.48 \pm 0.26 & 3.6 \pm 0.37 & 3.840 & \mathbf{0 . 0 2 9} & 0.667 & \mathbf{0 . 0 1 4} & \mathbf{0 . 0 2 6} & 0.745\end{array}$ $\begin{array}{lllllll}\text { Nonnormal } & 2.23 \pm 0.32 & 2.1 \pm 0.21 & 2.1 \pm 0.4 & 0.028 & 0.972 & 0.054\end{array}$ $\begin{array}{lllllll}\text { Nonnormal } & 2.38 \pm 0.33 & 2.17 \pm 0.29 & 2 \pm 0.36 & 0.011 & 0.989 & 0.051\end{array}$ Nonnormal $1.92+0.21 \quad 2.13+0.16 \quad 1.9+0.17 \quad 0.632 \quad 0.536 \quad 0.149$ $\begin{array}{lllllll}\text { Normal } & 12.25 \pm 0.59 & 11.98 \pm 0.49 & 12.22 \pm 0.53 & 0.117 & 0.890 & 0.067\end{array}$

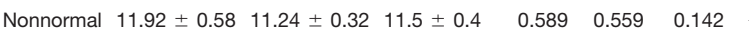

Gender effect Gender $\times$ genotype effect

$p$ value Power $F \quad p$ value Power

$\begin{array}{llllll}0.928 & 0.341 & 0.156 & 0.010 & 0.990 & 0.051\end{array}$ $\begin{array}{lllllll}0.774 & 0.384 & 0.138 & 0.929 & 0.403 & 0.201\end{array}$ $\begin{array}{llllll}0.010 & 0.922 & 0.051 & 1.615 & 0.210 & 0.323\end{array}$ $\begin{array}{llllll}1.030 & 0.316 & 0.168 & 3.307 & 0.046 & 0.597\end{array}$ $\begin{array}{llllll}0.718 & 0.401 & 0.132 & 1.010 & 0.372 & 0.215\end{array}$ $\begin{array}{lllllll}0.015 & 0.902 & 0.052 & 0.685 & 0.509 & 0.158\end{array}$ $\begin{array}{lllllll}0.421 & 0.520 & 0.097 & 5.349 & 0.008 & 0.815\end{array}$ $\begin{array}{lllllll}0.215 & 0.645 & 0.074 & 0.019 & 0.981 & 0.053\end{array}$ $\begin{array}{lllllll}2.068 & 0.158 & 0.290 & 0.043 & 0.958 & 0.056\end{array}$ $\begin{array}{llllll}1.782 & 0.189 & 0.257 & 1.179 & 0.317 & 0.245\end{array}$ $\begin{array}{lllllll}0.121 & 0.730 & 0.063 & 0.313 & 0.733 & 0.097\end{array}$ $\begin{array}{llllll}1.947 & 0.170 & 0.276 & 2.592 & 0.086 & 0.490\end{array}$ $\begin{array}{lllllll}0.044 & 0.834 & 0.055 & 0.205 & 0.816 & 0.080\end{array}$ $\begin{array}{llllll}0.046 & 0.831 & 0.055 & 1.755 & 0.185 & 0.348\end{array}$ $\begin{array}{llllll}0.701 & 0.407 & 0.130 & 0.297 & 0.745 & 0.094\end{array}$ 
Table 4. Continued

\begin{tabular}{|c|c|c|c|c|c|c|c|c|c|c|c|c|c|c|c|c|}
\hline \multicolumn{17}{|l|}{ Ultrasonic vocalizations } \\
\hline \multicolumn{2}{|c|}{ Repeated measures, sphericity violated } & $F$ & $p$ value & Power & $\begin{array}{l}\text { WT vs } \\
\text { Het }\end{array}$ & $\begin{array}{l}\text { WT vs } \\
\text { KO }\end{array}$ & $\begin{array}{l}\text { Het vs } \\
\text { KO }\end{array}$ & & & & & & & & & \\
\hline \multicolumn{2}{|l|}{ Genotype effect } & 0.533 & 0.590 & 0.133 & - & - & - & & & & & & & & & \\
\hline \multicolumn{2}{|l|}{ Gender effect } & 1.697 & 0.199 & 0.248 & - & - & - & & & & & & & & & \\
\hline \multirow{2}{*}{\multicolumn{2}{|c|}{ Genotype $\times$ gender }} & 0.869 & 0.426 & 0.191 & - & - & - & & & & & & & & & \\
\hline & & & & & & & & & & & \multicolumn{3}{|c|}{ Gender effect } & \multicolumn{3}{|c|}{$\begin{array}{l}\text { Gender } \times \text { genotype } \\
\text { effect }\end{array}$} \\
\hline \multicolumn{2}{|l|}{ Multifactiorial ANCOVA } & WT & Het & $\mathrm{KO}$ & $F$ & $\begin{array}{l}p \\
\text { value }\end{array}$ & Power & $\begin{array}{l}\text { WT vs } \\
\text { Het }\end{array}$ & $\begin{array}{l}\text { WT vs } \\
\text { KO }\end{array}$ & $\begin{array}{l}\text { Het vs } \\
\text { KO }\end{array}$ & $F$ & $\begin{array}{l}p \\
\text { value }\end{array}$ & Power & $F$ & $\begin{array}{l}p \\
\text { value }\end{array}$ & Power \\
\hline Number of calls - minute 1 & Nonnormal & $13.81 \pm 4.24$ & $11.46 \pm 2.89$ & $15.88 \pm 5.31$ & 0.586 & 0.562 & 0.139 & - & - & - & 0.163 & 0.689 & 0.068 & 0.090 & 0.914 & 0.063 \\
\hline Number of calls - minute 2 & Nonnormal & $18.68 \pm 5.15$ & $11.56 \pm 2.73$ & $13.22 \pm 4.78$ & 0.567 & 0.572 & 0.136 & - & - & - & 0.327 & 0.571 & 0.086 & 0.068 & 0.935 & 0.059 \\
\hline Number of calls - minute 3 & Nonnormal & $15.75 \pm 4.72$ & $13.96 \pm 3.72$ & $8.33 \pm 4.2$ & 0.172 & 0.843 & 0.074 & - & - & - & 3.481 & 0.071 & 0.442 & 0.097 & 0.908 & 0.064 \\
\hline Number of calls - total & Nonnormal & $48.43 \pm 13.28$ & $37.06 \pm 7.85$ & $37.44 \pm 12.08$ & 0.156 & 0.856 & 0.072 & - & - & - & 1.323 & 0.258 & 0.201 & 0.052 & 0.949 & 0.057 \\
\hline Calling time - minute 1 & Nonnormal & $0.93 \pm 0.29$ & $0.75 \pm 0.19$ & $1.08 \pm 0.38$ & 0.738 & 0.485 & 0.165 & - & - & - & 0.106 & 0.746 & 0.062 & 0.083 & 0.920 & 0.062 \\
\hline Calling time - minute 2 & Nonnormal & $1.24 \pm 0.39$ & $0.76 \pm 0.19$ & $0.85 \pm 0.3$ & 0.368 & 0.695 & 0.104 & - & - & - & 0.231 & 0.634 & 0.075 & 0.059 & 0.943 & 0.058 \\
\hline Calling time - minute 3 & Nonnormal & $1.16 \pm 0.4$ & $0.96 \pm 0.25$ & $0.53 \pm 0.27$ & 0.162 & 0.851 & 0.073 & - & - & - & 4.123 & 0.050 & 0.505 & 0.006 & 0.994 & 0.051 \\
\hline Calling time - total & Nonnormal & $3.41 \pm 1.05$ & $2.48 \pm 0.52$ & $2.47 \pm 0.81$ & 0.189 & 0.828 & 0.077 & - & - & - & 1.431 & 0.240 & 0.213 & 0.023 & 0.977 & 0.053 \\
\hline Average duration - $\min 1$ & Nonnormal & $0.05 \pm 0$ & $0.05 \pm 0$ & $0.03 \pm 0.01$ & 0.138 & 0.872 & 0.069 & - & - & - & 0.056 & 0.815 & 0.056 & 0.950 & 0.400 & 0.197 \\
\hline Average duration - $\min 2$ & Nonnormal & $0.06 \pm 0$ & $0.06 \pm 0$ & $0.03 \pm 0.01$ & 0.319 & 0.730 & 0.095 & - & - & - & 1.879 & 0.182 & 0.262 & 1.561 & 0.229 & 0.301 \\
\hline Average duration - $\min 3$ & Nonnormal & $0.06 \pm 0$ & $0.06 \pm 0$ & $0.02 \pm 0.01$ & 6.759 & 0.004 & 0.883 & - & - & - & 23.838 & 0.000 & 0.997 & 7.350 & 0.003 & 0.909 \\
\hline Average duration - total & Nonnormal & $0.08 \pm 0.01$ & $0.06 \pm 0$ & $0.03 \pm 0.01$ & 0.515 & 0.604 & 0.125 & - & - & - & 1.663 & 0.209 & 0.237 & 0.847 & 0.440 & 0.179 \\
\hline Latency to first call & Nonnormal & $77.28 \pm 17.98$ & $80.04 \pm 12.54$ & $75.57 \pm 27.65$ & 0.411 & 0.665 & 0.113 & - & - & - & 0.155 & 0.696 & 0.067 & 3.311 & 0.045 & 0.602 \\
\hline Mean frequency - total & Nonnormal & $71337.77 \pm 1902.04$ & $73128.73 \pm 3879.33$ & $75883.81 \pm 3957.75$ & 0.024 & 0.976 & 0.053 & - & - & - & 0.036 & 0.851 & 0.054 & 0.029 & 0.971 & 0.054 \\
\hline Mean amplitude - total & Nonnormal & $78.43 \pm 29.11$ & $66.33 \pm 20.80$ & $37.15 \pm 17.64$ & 0.189 & 0.829 & 0.076 & - & - & - & 2.440 & 0.130 & 0.325 & 0.442 & 0.648 & 0.114 \\
\hline Percentage of noncaller & Nonnormal & $18.75 \pm 10.08$ & $28.13 \pm 8.08$ & $33.33 \pm 16.67$ & 0.401 & 0.672 & 0.111 & - & - & - & 0.587 & 0.447 & 0.117 & 1.933 & 0.155 & 0.382 \\
\hline
\end{tabular}

WT, wild-type mice; Het, heterozygous mice; KO, homozygous knock-out mice. Group values are reported as mean \pm SEM. Bold font indicates significant results $(p<0.05)$. P: postnatal day.

gous animals (Fig. $3 A$ ) and reduced spontaneous locomotion was observed during a 1-h open field session in both Shank $3^{\Delta 4-22}$ heterozygous and homozygous mice (Fig. $3 B$ ). Across the 60 -min session, the time course for total distance traversed by all three genotypes declined as expected, representing habituation to the open field. However, while the distance traveled during the first 10 min was similar for the three groups, the decline was faster for Shank $3^{\Delta 4-22}$ homozygous mice, possibly reflecting a higher fatigability. Similarly, in the accelerating rotarod test, which assay for gait, balance, motor coordination and endurance, shorter latencies to fall where observed in Shank $3^{\Delta 4-22}$-deficient mice after the first trial, with a milder phenotype observed in the heterozygotes compared to homozygotes. When examining learning in this paradigm, characterized by an improvement of performance (latency to fall) over the trials, Shank $3^{\Delta 4-22}$ heterozygous and homozygous animals failed to improve over time, in contrast to wild-type animals which showed typical learning (Fig. 3C).

Impairment of motor coordination and balance was also observed in Shank $3^{\Delta 4-22}$ homozygous in the beam walking test (Fig. 3D; Table 6) as well by reduced strength and endurance in both the inverted screen and hanging tests (Fig. 3E), but with no differences in forelimb grip strength (Extended Data Fig. 3-1A). There was also a trend toward an increased number of failed attempt in the hindlimb placing for Shank $3^{\Delta 4-22}$ homozygous mice, compared to their littermates (Extended Data Fig. 3-1B).

\section{Sensory abilities in Shank $3^{\Delta 4-22}$-deficient mice}

For all sensory-related assays, detailed results are reported in Table 7.

No genotype differences were detected in tactile tests including the pinna reflex, the palpebral reflex, and the toe pinch retraction test. In the tail flick pain sensitivity test, a trend toward a decreased latency to flick the tail in re- sponse to a noxious thermal stimulation a was observed in Shank3 $3^{\Delta-22}$ homozygous animals (Fig. 4A).

Normal Preyer reflexes were observed in all genotypes; however, Shank $3^{\Delta 4-22}$ heterozygous and homozygous mice showed a reduced startle response throughout all the sound intensities (74-92 dB, analyzed as repeated measures) indicating an impaired sound discrimination (Fig. 4B). Changes in pre-pulse inhibition of acoustic startle in Shank $3^{\Delta 4-22}$-deficient mice are consistent with abnormalities in auditory processing, rather than sensorimotor gating deficits (Extended Data Fig. 4-1A).

Normal visual placing/reaching reflexes were observed for all the mice, thus ruling out strong visual impairments (Fig. 4C).

Shank $3^{\Delta 4-22}$ homozygous mice demonstrated strong deficits in the buried food test (Fig. $4 D$, left panel) with only seven out of 19 mice able to retrieve the food in less than 2 min and nine out of 19 mice not being able to find the food at all (Extended Data Fig. 4-1B). However, all animals showed interest for the food and ate it when it was made visible. To further investigate olfactory function, animals were subjected to the olfactory habituation/dishabituation paradigm using three nonsocial scents (water, banana, and lemon) and two social scents (unfamiliar males and unfamiliar females). Wild-type and Shank $3^{\Delta 4-22}$ heterozygous animals displayed a normal response, characterized by a robust sniffing elicited by the first scent presentation of each nonsocial and social scent that declined over the second and third presentation of the same scent. In contrast, Shank $3^{\Delta 4-22}$ homozygous animals had little response to any of the nonsocial scents, even on their first presentation (Fig. $4 D$, middle panel), thus confirming the results of the buried food test. Interestingly the lack of interest for olfactory stimuli does not appear to be the consequence of anosmia as a normal response to both social scents was observed in Shank $3^{\Delta 4-22}$ homozygous mice (Fig. $4 D$, right panel). 
A

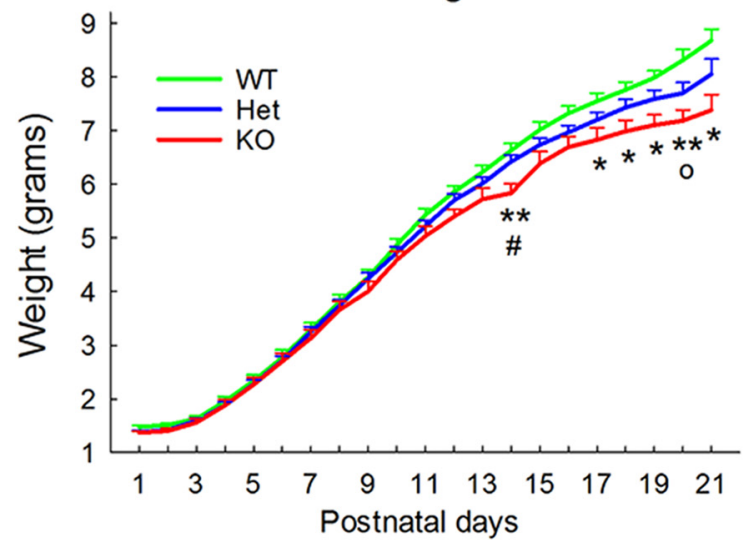

C

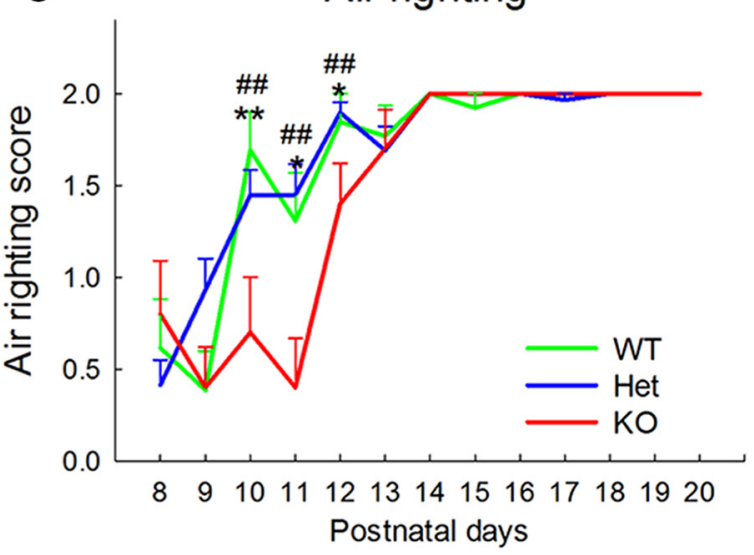

E

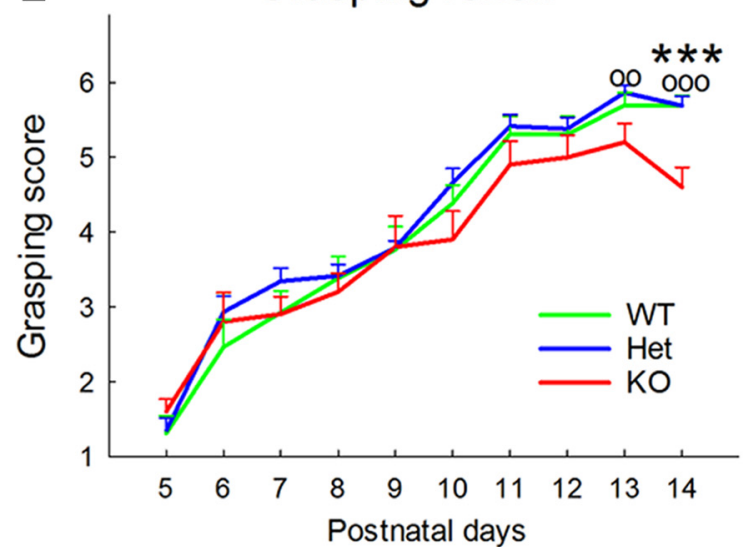

B

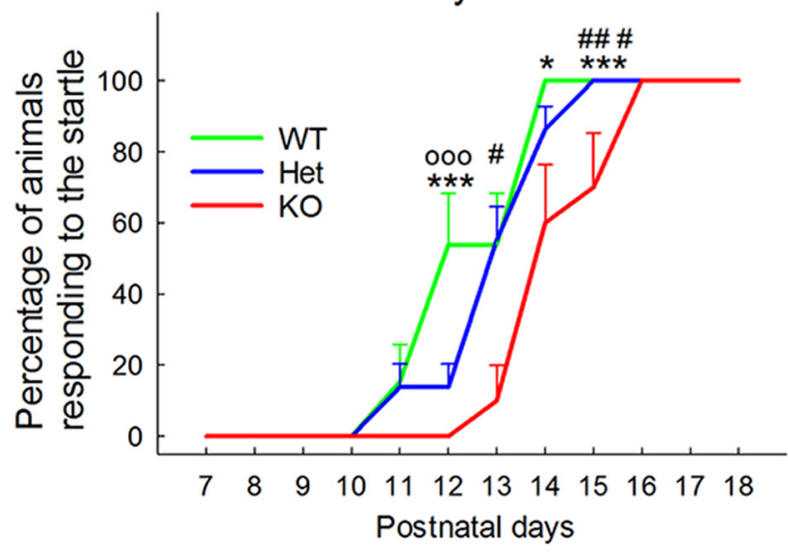

D

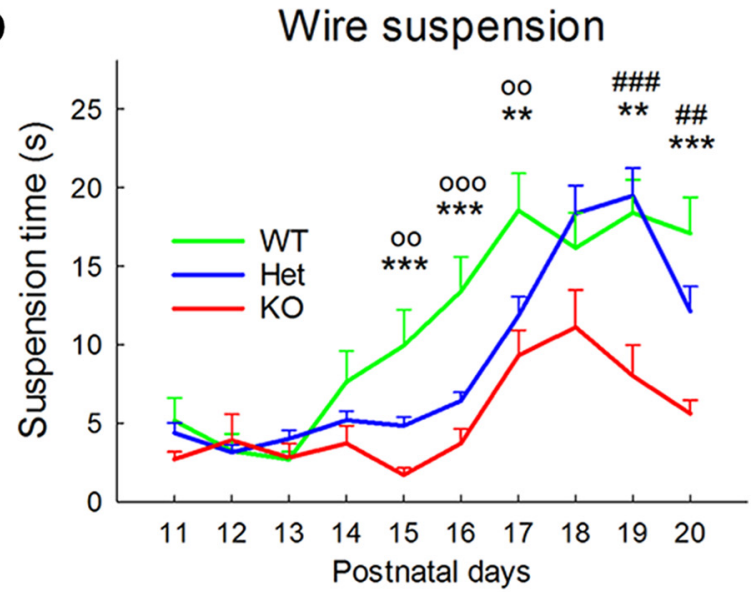

F

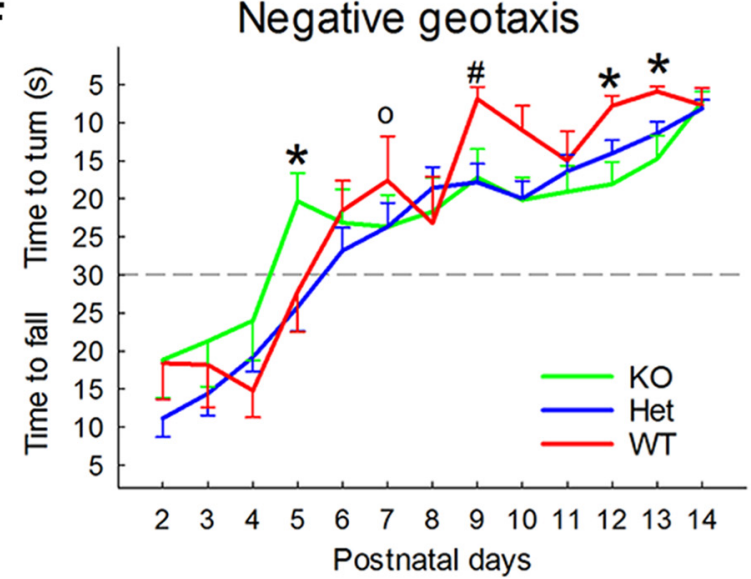

Figure 2. Delayed developmental milestones of in Shank $3^{\Delta 4-22}$-deficient mice. Analysis of markers of developmental milestones revealed genotype differences in Shank $3^{\Delta 4-22}$ wild-type, heterozygous, and homozygous pups between postnatal days 1 and 21 on measures of $(\boldsymbol{A})$ body weight, $(\boldsymbol{B})$ auditory startle, $(\boldsymbol{C})$ air righting, $(\boldsymbol{D})$ wire suspension, $(\boldsymbol{E})$ grasping reflex, and $(\boldsymbol{F})$ negative geotaxis. Additional milestones (jar opening, tooth eruption, fur development, eye opening, rooting reflex, cliff aversion, ear twitch, surface righting, open field crossing, and ultrasonic vocalizations) are displayed in Extended Data Figure 2-1. WT, wild-type mice; Het, heterozygous mice; $\mathrm{KO}$, homozygous knock-out mice. $*$ : WT versus KO; o: WT versus Het, \#: Het versus KO. $* p<0.05, * * p<0.1$, $* * * p<0.001$.

\section{Social interactions in Shank3 ${ }^{\Delta 4-22}$-deficient mice}

Mice were evaluated for social abilities during malefemale dyadic social interaction, in the three-chambered social interaction task, and in the social transmission of food preference test and detailed results are reported in Table 8. In freely moving male-female dyads of male mice paired with unfamiliar wild-type estrous C57BL6 females, sniffing time was generally similar across genotypes (Fig. 
Table 5. Detailed results and statistical analyses related to general health, physical factors, gross appearance, and spontaneous activity

\begin{tabular}{|c|c|c|c|c|c|c|c|c|c|c|c|c|c|c|c|c|c|}
\hline \multicolumn{18}{|c|}{ Physical factors and gross appearance } \\
\hline & \multirow[b]{2}{*}{ Test } & \multirow[b]{2}{*}{$\begin{array}{l}\text { Data } \\
\text { structure }\end{array}$} & \multirow[b]{2}{*}{ WT } & \multirow[b]{2}{*}{ Het } & \multirow[b]{2}{*}{$\mathrm{KO}$} & \multicolumn{3}{|c|}{ Genotype } & \multicolumn{3}{|l|}{ Cohort } & \multicolumn{2}{|c|}{$\begin{array}{l}\text { Genotype } \times \\
\text { cohort }\end{array}$} & \multirow[b]{2}{*}{ Power } & \multicolumn{3}{|c|}{$\begin{array}{l}\text { Pairwise } \\
\text { comparisons }\end{array}$} \\
\hline & & & & & & $F$ & $\begin{array}{l}p \\
\text { value }\end{array}$ & Power & $F$ & $\begin{array}{l}p \\
\text { value }\end{array}$ & Power & $F$ & $\begin{array}{l}p \\
\text { value }\end{array}$ & & $\begin{array}{l}\text { WT vs } \\
\text { Het }\end{array}$ & $\begin{array}{l}\text { WT vs } \\
\text { KO }\end{array}$ & $\begin{array}{l}\text { Het vs } \\
\text { KO }\end{array}$ \\
\hline 3-months weight (g) & 2WANOVA & Normal & $26.33 \pm 0.87$ & $27.18 \pm 0.57$ & $26.01 \pm 0.54$ & 1.241 & 0.298 & 0.258 & 2.546 & 0.117 & 0.347 & 2.000 & 0.146 & 0.394 & - & - & - \\
\hline 15-months weight (g) & 2WANOVA & Normal & $33.41 \pm 2$ & $31.06 \pm 1.21$ & $29.36 \pm 1.73$ & 1.578 & 0.222 & 0.310 & 0.269 & 0.608 & 0.079 & 0.491 & 0.617 & 0.123 & - & - & - \\
\hline 20-months weight (g) & 2WANOVA & Normal & $32.9 \pm 1.86$ & $31.43 \pm 1.2$ & $28.84 \pm 1.5$ & 0.982 & 0.390 & 0.199 & 0.034 & 0.856 & 0.054 & 0.018 & 0.982 & 0.052 & - & - & - \\
\hline Length & 2WANOVA & Nonnormal & $16.45 \pm 0.24$ & $16.84 \pm 0.24$ & $16.73 \pm 0.25$ & 1.062 & 0.353 & 0.226 & 91.207 & 0.000 & 1.000 & 0.471 & 0.627 & 0.123 & - & - & - \\
\hline Coat appearance & 2WANOVA & Nonnormal & $2.63 \pm 0.13$ & $2.89 \pm 0.07$ & $2.94 \pm 0.05$ & 2.558 & 0.087 & 0.489 & 2.615 & 0.112 & 0.355 & 1.424 & 0.250 & 0.291 & 0.119 & 0.050 & 0.915 \\
\hline Skin color & - & - & $0 \pm 0$ & $0 \pm 0$ & $0 \pm 0$ & - & - & - & - & - & - & - & - & - & - & - & - \\
\hline Whisker barbering & - & - & $0 \pm 0$ & $0 \pm 0$ & $0 \pm 0$ & - & - & - & - & - & - & - & - & - & - & - & - \\
\hline Missing fur on face & - & - & $0 \pm 0$ & $0 \pm 0$ & $0 \pm 0$ & - & - & - & - & - & - & - & - & - & - & - & - \\
\hline Missing fur on body & - & - & $0 \pm 0$ & $0 \pm 0$ & $0 \pm 0$ & - & - & - & - & - & - & - & - & - & - & - & - \\
\hline Wounding & 2WANOVA & Nonnormal & $0.15 \pm 0.11$ & $0 \pm 0$ & $0.05 \pm 0.05$ & 1.078 & 0.348 & 0.229 & 0.028 & 0.869 & 0.053 & 0.279 & 0.758 & 0.092 & - & - & - \\
\hline Body tone & 2WANOVA & Nonnormal & $1.1 \pm 0.07$ & $1.1 \pm 0.07$ & $1 \pm 0.07$ & 0.563 & 0.573 & 0.138 & 2.225 & 0.142 & 0.310 & 0.563 & 0.573 & 0.138 & - & - & - \\
\hline Palpebral closure & - & - & $0 \pm 0$ & $0 \pm 0$ & $0 \pm 0$ & - & - & - & - & - & - & - & - & - & - & - & - \\
\hline Piloerection & - & - & $0 \pm 0$ & $0 \pm 0$ & $0 \pm 0$ & - & - & - & - & - & - & - & - & - & - & - & - \\
\hline \multicolumn{18}{|l|}{ Jar observation } \\
\hline & & & & & & \multicolumn{3}{|c|}{ Genotype } & \multicolumn{3}{|l|}{ Cohort } & \multicolumn{3}{|c|}{$\begin{array}{l}\text { Genotype } \times \\
\text { cohort }\end{array}$} & \multicolumn{3}{|c|}{$\begin{array}{l}\text { Pairwise } \\
\text { comparisons }\end{array}$} \\
\hline & Test & $\begin{array}{l}\text { Data } \\
\text { structure }\end{array}$ & WT & Het & $\mathrm{KO}$ & $F$ & $\begin{array}{l}p \\
\text { value }\end{array}$ & Power & $F$ & $\begin{array}{l}p \\
\text { value }\end{array}$ & Power & $F$ & $\begin{array}{l}p \\
\text { value }\end{array}$ & Power & $\begin{array}{l}\text { WT vs } \\
\text { Het }\end{array}$ & $\begin{array}{l}\text { WT vs } \\
\text { KO }\end{array}$ & $\begin{array}{l}\text { Het vs } \\
\mathrm{KO}\end{array}$ \\
\hline Body position & 2WANOVA & Nonnormal & $4.15 \pm 0.08$ & $4.1 \pm 0.07$ & $4.26 \pm 0.1$ & 0.702 & 0.500 & 0.162 & 4.949 & 0.031 & 0.588 & 0.139 & 0.871 & 0.070 & - & - & - \\
\hline Spontaneous activity & 2WANOVA & Nonnormal & $1.68 \pm 0.1$ & $1.57 \pm 0.11$ & $1.63 \pm 0.11$ & 0.223 & 0.801 & 0.083 & 0.282 & 0.598 & 0.082 & 0.279 & 0.758 & 0.092 & - & - & - \\
\hline Latency to sit/stand (s) & - & - & $0 \pm 0$ & $0 \pm 0$ & $0 \pm 0$ & - & - & - & - & - & - & - & - & - & - & - & - \\
\hline Latency to rear (s) & 2WANOVA & Nonnormal & $8.94 \pm 1.36$ & $8.05 \pm 1.03$ & $5.63 \pm 1.02$ & 2.137 & 0.128 & 0.418 & 0.036 & 0.850 & 0.054 & 0.046 & 0.955 & 0.057 & - & - & - \\
\hline Repeated jumps (\%) & 2WANOVA & Nonnormal & $15.78 \pm 8.59$ & $10.52 \pm 7.23$ & $26.31 \pm 10.37$ & 0.702 & 0.500 & 0.162 & 4.949 & 0.031 & 0.588 & 0.139 & 0.871 & 0.070 & - & - & - \\
\hline Circling (\%) & 2WANOVA & Nonnormal & $5.26 \pm 5.26$ & $10.52 \pm 7.23$ & $10.52 \pm 7.23$ & 0.289 & 0.750 & 0.093 & 5.177 & 0.027 & 0.607 & 0.289 & 0.750 & 0.093 & - & - & - \\
\hline Urination & 2WANOVA & Nonnormal & $0.47 \pm 0.19$ & $0.1 \pm 0.1$ & $0.15 \pm 0.11$ & 1.540 & 0.224 & 0.312 & 0.023 & 0.881 & 0.052 & 1.213 & 0.306 & 0.253 & - & - & - \\
\hline Defecation (number) & 2WANOVA & Nonnormal & $1.57 \pm 0.35$ & $0.94 \pm 0.29$ & $0.94 \pm 0.27$ & 1.065 & 0.352 & 0.226 & 0.003 & 0.958 & 0.050 & 2.592 & 0.085 & 0.494 & - & - & - \\
\hline Respiration & - & - & $2 \pm 0$ & $2 \pm 0$ & $2 \pm 0$ & - & - & & - & - & & - & - & & - & - & - \\
\hline Tremor & - & - & $0 \pm 0$ & $0 \pm 0$ & $0 \pm 0$ & - & - & & - & - & & - & - & & - & - & - \\
\hline \multicolumn{18}{|l|}{ Cage transfer } \\
\hline & & & & & & \multicolumn{3}{|c|}{ Genotype } & \multicolumn{3}{|l|}{ Cohort } & \multicolumn{3}{|c|}{$\begin{array}{l}\text { Genotype } \times \\
\text { cohort }\end{array}$} & \multicolumn{3}{|c|}{$\begin{array}{l}\text { Pairwise } \\
\text { comparisons }\end{array}$} \\
\hline & Test & $\begin{array}{l}\text { Data } \\
\text { structure }\end{array}$ & WT & Het & $\mathrm{KO}$ & $F$ & $\begin{array}{l}p \\
\text { value }\end{array}$ & Power & $F$ & $\begin{array}{l}p \\
\text { value }\end{array}$ & Power & $F$ & $\begin{array}{l}p \\
\text { value }\end{array}$ & Power & $\begin{array}{l}\text { WT vs } \\
\text { Het }\end{array}$ & $\begin{array}{l}\text { WT vs } \\
\text { KO }\end{array}$ & $\begin{array}{l}\text { Het vs } \\
\text { KO }\end{array}$ \\
\hline Transfer arousal & 2WANOVA & Nonnormal & $3.21 \pm 0.22$ & $3.21 \pm 0.22$ & $3.15 \pm 0.2$ & 0.037 & 0.964 & 0.055 & 1.470 & 0.231 & 0.221 & 2.055 & 0.139 & 0.404 & - & - & - \\
\hline Gait & 2WANOVA & Nonnormal & $0 \pm 0$ & $0.15 \pm 0.08$ & $0.05 \pm 0.05$ & 1.783 & 0.178 & 0.356 & 0.003 & 0.957 & 0.050 & 0.637 & 0.533 & 0.151 & - & - & - \\
\hline Pelvic elevation & 2wANOVA & Nonnormal & $2 \pm 0$ & $2.15 \pm 0.08$ & $2 \pm 0.07$ & 1.730 & 0.188 & 0.347 & 0.141 & 0.709 & 0.066 & 0.141 & 0.869 & 0.071 & - & - & - \\
\hline Tail elevation & 2WANOVA & Nonnormal & $1.89 \pm 0.15$ & $1.73 \pm 0.18$ & $1.21 \pm 0.18$ & 4.003 & 0.024 & 0.691 & 4.469 & 0.039 & 0.545 & 0.204 & 0.816 & 0.080 & 0.530 & 0.009 & 0.043 \\
\hline
\end{tabular}

WT, wild-type mice; Het, heterozygous mice; KO, homozygous knock-out mice. Group values are reported as mean \pm SEM. Bold font indicates significant results $(p<0.05)$. Individual results and statistical analyses for cohorts 1 and 2 are available in Extended Data Table 5-1. 2wANOVA: 2-way ANOVA.

$5 A$, left panel). A significant increase in latency for the first event of anogenital sniffing was found in male Shank $3^{\Delta 4-22}$ homozygous mice (Fig. $5 A$, right panel), and we can note that this latency may contribute to trend toward reduced anogenital sniffing time in those animals. Ultrasonic vocalizations did not show significant difference across genotypes (Extended Data Fig. 5-1A).

Similarly, In the three-chambered test for social preference, sociability, defined as spending more time interacting with the mouse than with the object, was found in all genotypes. Hence, in all groups, significantly more time was spent in the chamber containing the novel mouse than in the chamber containing the novel object, and more time was spent sniffing the novel mouse than the novel object (Fig. 5B). All genotypes showed the normal absence of innate chamber side bias during the 10-min habituation phase before the start of the sociability test.

Finally, mice were tested in the social transmission of food preference test that combines social behavior, olfactory recognition and memory skills. A modest decrease of the number of sniffing bouts initiated by the observer mouse toward the demonstrator mouse was observed during the observer-demonstrator interaction phase in
Shank $3^{\Delta 4-22}$ homozygous mice but not in heterozygotes (Extended Data Fig. 5-1B). All genotypes showed a strong preference for the cued food flavor that was exposed to them through the demonstrator, as compared to the noncued food flavor, as shown both by significantly more time spent interacting with the jar containing the cued food than the noncued food (Fig. 5C) or by eating significantly more cued food than noncued food during the choice phase (Table 8). Note that two flavors were randomly used as cued and noncued food flavor and all genotypes showed an absence of flavor preference. However, the total amount of food (cued and noncued) eaten by Shank $3^{\Delta-22}$ homozygous mice was significantly lower than the total amount of food eaten by their wild-type and homozygous littermates.

\section{Object avoidance in Shank ${ }^{\mathbf{4 - 2 2}}$-deficient mice}

While testing mice in different set-ups involving object interactions, a strong avoidance toward inanimate objects was observed in Shank $3^{\Delta 4-22}$ homozygous mice (Table 9).

This avoidance behavior was initially observed in the novel object recognition task. This highly validated test for recognition memory is designed to evaluate differences in the exploration time of novel and familiar objects. Mice 
Table 6. Detailed results and statistical analyses related to motor functions

\begin{tabular}{|c|c|c|}
\hline \multicolumn{3}{|l|}{ Gait analysis } \\
\hline & Test & $\begin{array}{l}\text { Data } \\
\text { structure }\end{array}$ \\
\hline Stance mean $(\mathrm{cm})$ & 2WANOVA & Normal \\
\hline Stance variance $(\mathrm{cm})$ & 2WANOVA & Nonnorm \\
\hline Stride mean $(\mathrm{cm})$ & 2WANOVA & Normal \\
\hline Stride variance $(\mathrm{cm})$ & 2WANOVA & Nonnorm \\
\hline Sway mean $(\mathrm{cm})$ & 2WANOVA & Nonnorm \\
\hline Sway variance $(\mathrm{cm})$ & 2WANOVA & Nonnorm \\
\hline \multicolumn{3}{|c|}{ Open field spontaneous activity (traveled } \\
\hline & Test & $\begin{array}{l}\text { Data } \\
\text { structure }\end{array}$ \\
\hline Total distance $(\mathrm{cm})$ & 2WANOVA & Normal \\
\hline Distance & Test & $\begin{array}{l}\text { Data } \\
\text { structure }\end{array}$ \\
\hline - Time & rMeasures & Sph.viol \\
\hline - Time $\times$ gen & rMeasures & Sph.viol \\
\hline - Genotype & rMeasures & Sph.viol \\
\hline - Cohort & rMeasures & Sph.viol \\
\hline - Time $\times$ gen. $\times$ coh . & rMeasures & Sph.viol \\
\hline - gen. $\times$ coh. & rMeasures & Sph.viol \\
\hline
\end{tabular}

\begin{tabular}{|c|c|c|}
\hline Individual time bins & Test & $\begin{array}{l}\text { Data } \\
\text { structure }\end{array}$ \\
\hline Distance $0-10 \mathrm{~min}$ & 2WANOVA & Normal \\
\hline Distance $10-20 \mathrm{~min}$ & 2WANOVA & Nonnorme \\
\hline Distance $20-30 \mathrm{~min}$ & 2WANOVA & Normal \\
\hline Distance $30-40 \mathrm{~min}$ & 2WANOVA & Normal \\
\hline Distance $40-50 \mathrm{~min}$ & 2WANOVA & Normal \\
\hline Distance $50-60 \mathrm{~min}$ & 2WANOVA & Nonnorme \\
\hline \multicolumn{3}{|l|}{ Rotarod } \\
\hline Latency & Test & $\begin{array}{l}\text { Data } \\
\text { structure }\end{array}$ \\
\hline - Trial & rMeasures & Sph.viol \\
\hline - Trial $\times$ gen. & rMeasures & Sph.viol \\
\hline - Genotype & rMeasures & Sph.viol \\
\hline - Cohort & rMeasures & Sph.viol \\
\hline $\begin{array}{l}\text { - Session } \times \text { gen. } \\
\quad \times \text { coh. }\end{array}$ & rMeasures & Sph.viol \\
\hline - Gen. $\times$ coh. & rMeasures & Sph.viol \\
\hline
\end{tabular}

Individual trials

Latency trial 1 Latency trial 2 Latency trial 3

Latency trial 4 Latency trial 5 Latency trial 6 Latency day 1 Latency day 2

Beam walking
Het KO

$3.41 \pm 0.12$

$0.15 \pm 0.03$

$5.64 \pm 0.21$

$0.86 \pm 0.14$

$3.55 \pm 0.16$

$0.17 \pm 0.04$
$3.27 \pm 0.14$

$0.17 \pm 0.05$

$6.43 \pm 0.24$

$0.83 \pm 0.18$

$3.59 \pm 0.22$

$0.14 \pm 0.02$

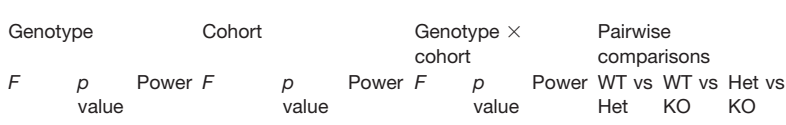

$\begin{array}{llllllllll}1.466 & 0.240 & 0.299 & 40.902 & 0.000 & 1.000 & 1.291 & 0.284 & 0.267 & -\end{array}$

$\begin{array}{llllllllllll}0.189 & 0.829 & 0.078 & 14.972 & 0.000 & 0.967 & 0.735 & 0.484 & 0.168 & -\end{array}$

$\begin{array}{llllllllllll}5.443 & 0.007 & 0.826 & 15.476 & 0.000 & 0.971 & 0.499 & 0.610 & 0.127 & 0.674 & \mathbf{0 . 0 0 3} & \mathbf{0 . 0 2 8}\end{array}$

$\begin{array}{llllllllll}0.137 & 0.873 & 0.070 & 12.150 & 0.001 & 0.928 & 3.459 & 0.039 & 0.622 & -\end{array}$

$\begin{array}{llllllllll}0.191 & 0.826 & 0.078 & 186.368 & 0.000 & 1.000 & 2.443 & 0.097 & 0.470-\end{array}$

$\begin{array}{lllllllllll}1.909 & 0.159 & 0.378 & 3.781 & 0.057 & 0.479 & 0.400 & 0.672 & 0.111\end{array}$
Genotype Cohort Genotype $\times \quad$ Pairwise

Het

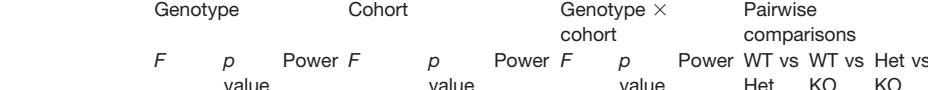

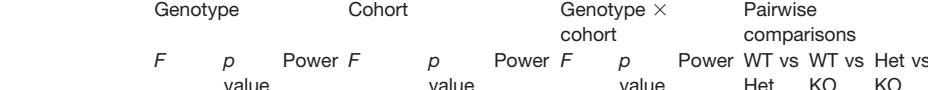
$13,816.17 \pm 828.2711,273.16 \pm 764.0910,099.24 \pm 621.43$

\section{F $\quad p \quad$ Power WT vs WT vs Het vs \\ $\begin{array}{llll}F & P & \text { Power WT vs } & \text { WT vs Het v } \\ \text { value } & \text { Het } & \text { KO KO }\end{array}$ \\ 36.3500 .0001 .000 \\ $\begin{array}{llll}2.235 & 0.029 & 0.917\end{array}$ \\ $\begin{array}{llllll}6.633 & 0.003 & 0.896 & 0.029 & 0.001 & 0.449\end{array}$ \\ 12.8360 .0010 .940 \\ $\begin{array}{llll}0.878 & 0.532 & 0.461\end{array}$ \\ $\begin{array}{llll}0.073 & 0.930 & 0.061\end{array}$}

Genotype Cohort Genotype $\times \quad$ Pairwise cohort comparisons

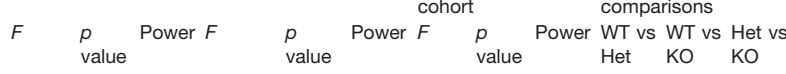
$\mathrm{KO}$ $2723.06 \pm 185.28 \quad 2365.11 \pm 166.4$ $2516.77 \pm 150.09 \quad 2064.33 \pm 169.75$ $2349.52 \pm 168.15 \quad 1919.27 \pm 159.57$ $2203.27 \pm 139.79 \quad 1680.09 \pm 143.29$ $2090.38 \pm 156.71 \quad 1657.47 \pm 139.76$ $1586.87 \pm 98.6$ $\begin{array}{llllllllllll}2479.64 & \pm 164.36 & 0.894 & 0.415 & 0.196 & 7.573 & 0.008 & 0.770 & 0.224 & 0.800 & 0.083 & -\end{array}$

$\begin{array}{llllllllllllll}1684.88 & \pm 108.32 & 8.357 & 0.001 & 0.954 & 13.148 & 0.001 & 0.945 & 0.026 & 0.975 & 0.054 & 0.030 & 0.000 & 0.069\end{array}$ $\begin{array}{llllllllllllll}1466.99+150.82 & 7.936 & 0.001 & 0.943 & 11.962 & 0.001 & 0.924 & 0.362 & 0.698 & 0.105 & 0.051 & 0.000 & 0.051\end{array}$ $\begin{array}{llllllllllllll}1589.41 \pm 114.03 & 6.091 & \mathbf{0 . 0 0 4} & 0.868 & 11.521 & \mathbf{0 . 0 0 1} & 0.915 & 0.020 & 0.980 & 0.053 & \mathbf{0 . 0 0 7} & \mathbf{0 . 0 0 2} & 0.710\end{array}$ $\begin{array}{lllllllllllllll}1380 \pm 116.11 & 6.255 & \mathbf{0 . 0 0 4} & 0.877 & 5.904 & \mathbf{0 . 0 1 9} & 0.664 & 0.035 & 0.965 & 0.055 & 0.035 & \mathbf{0 . 0 0 1} & 0.185\end{array}$

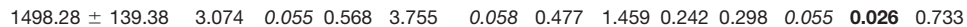

\footnotetext{
$\begin{array}{llll} & \text { Test } & \begin{array}{l}\text { Data } \\ \text { structure }\end{array} & \text { WT } \\ \text { \% mice falling (large) } & \text { 2WANOVA NA } & 0 \pm 0\end{array}$

$\%$ mice falling 2WANOVA Nonnormal $0 \pm 0$ (medium)

$\%$ mice falling (small) 2 wANOVA Nonnormal $32.89 \pm 7.41$ Distance $($ large, $\mathrm{cm}) \quad$ 2wANOVA Nonnormal $95.05 \pm 2.27$ Distance (medium, cm) 2WANOVA Nonnormal $87.17 \pm 4.25$ Distance (small, cm) 2WANOVA Normal $\quad 47.19 \pm 5.45$

$\%$ mice fully crossing 2 wANOVA Nonnormal $94.73 \pm 2.4$ (large)

$\%$ mice fully crossing 2 WANOVA Nonnormal $76.31 \pm 7.01$ (medium)

$\%$ mice fully crossing 2 WANOVA Nonnormal $27.63 \pm 7.12$
}

Data WANOVA Normal 2WANOVA Norma 2WANOVA Norma 2WANOVA Normal 2WANOVA Norma 2WANOVA Norma 2WANOVA Norma 2WANOVA Norma
WT

$181.28 \pm 15.25$ $222.75+20.99$ $260.92 \pm 18.73$ $270.8 \pm 21.62$ $273.51 \pm 19.16$ $200.9 \pm 15.13$ $268.41 \pm 16.68$
Het

$175.51 \pm 14.58$ $190.76 \pm 20.22$ $178.3 \pm 14.43$ $209.71 \pm 15.54$ $228.95 \pm 22.25$ $192.29 \pm 25.69$ $181.52 \pm 14.32$ $210.32+19.07$
KO

$149.17 \pm 13.2$ $135.84 \pm 15.82$ $128.94 \pm 14.01$ $168.01 \pm 16.99$ $172.55 \pm 21.59$ $133.8 \pm 15.59$ $137.98 \pm 11.9$ $158.12 \pm 14.54$

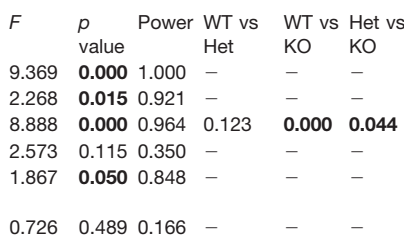
$\begin{array}{llllllllll}0.819 & 0.056 & 1.320 & 0.276 & 0.273 & - & - & - & -\end{array}$ $\begin{array}{llllllll}0.979 & 0.050 & 3.956 & 0.025 & 0.685 & 0.947 & 0.042 & 0.085\end{array}$ $\begin{array}{llllllll}0.311 & 0.171 & 0.816 & 0.448 & 0.182 & 0.159 & 0.001 & 0.106\end{array}$ $\begin{array}{llllllll}0.056 & 0.484 & 0.017 & 0.983 & 0.052 & 0.094 & 0.001 & 0.203\end{array}$ $\begin{array}{llllllll}0.221 & 0.229 & 1.050 & 0.357 & 0.224 & 0.368 & 0.007 & 0.168\end{array}$ $\begin{array}{llllllll}0.004 & 0.838 & 0.222 & 0.802 & 0.083 & 0.013 & 0.000 & 0.094\end{array}$ $\begin{array}{llllllll}0.744 & 0.062 & 2.253 & 0.115 & 0.438 & 0.578 & 0.006 & 0.072\end{array}$ $\begin{array}{lllllllll}0.020 & 0.655 & 0.060 & 0.942 & 0.059 & 0.041 & 0.000 & 0.073\end{array}$ value value Het KO KO
Genotype Cohort Genotype $\times \quad$ Pairwise

$F \quad p \quad$ Power $F \quad p \quad$ Power $F \quad p \quad$ Power WT vs WT vs Het vs

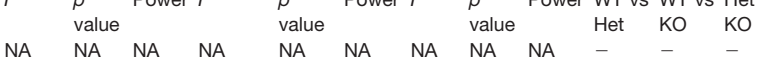
$\begin{array}{llllllllllll}\text { NA } & \text { NA } & \text { NA } & \text { NA } & \text { NA } & \text { NA } & \text { NA } & \text { NA } & \text { NA } & - & - & - \\ 6.339 & 0.003 & 0.882 & 0.395 & 0.533 & 0.095 & 0.396 & 0.675 & 0.111 & 1.000 & \mathbf{0 . 0 0 3} & \mathbf{0 . 0 0 3}\end{array}$

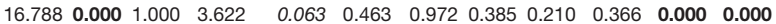
$\begin{array}{lllllllllll}1.819 & 0.173 & 0.362 & 1.416 & 0.240 & 0.215 & 0.242 & 0.786 & 0.086\end{array}$

$\begin{array}{llllllllll}0.129 & 0.879 & 0.069 & 2.525 & 0.118 & 0.344 & 0.482 & 0.620 & 0.125\end{array}$

$\begin{array}{lllllllllllll}4.380 & 0.018 & 0.732 & 0.447 & 0.507 & 0.101 & 0.346 & 0.709 & 0.102 & 0.438 & 0.044 & 0.006\end{array}$

$\begin{array}{llllllllllllll}1.567 & 0.219 & 0.317 & 1.486 & 0.228 & 0.223 & 0.107 & 0.899 & 0.066 & -\end{array}$

$\begin{array}{llllllllll}0.089 & 0.915 & 0.063 & 4.278 & 0.044 & 0.528 & 0.947 & 0.394 & 0.205 & -\end{array}$

$\begin{array}{llllllllllll}3.568 & 0.035 & 0.637 & 0.692 & 0.409 & 0.129 & 0.316 & 0.730 & 0.098 & 0.278 & 0.128 & 0.010\end{array}$ 
Table 6. Continued

\begin{tabular}{|c|c|c|c|c|c|c|c|c|c|c|c|c|c|c|c|c|c|}
\hline \multirow[b]{2}{*}{ Individual trials } & \multirow[b]{2}{*}{ Test } & \multirow[b]{2}{*}{$\begin{array}{l}\text { Data } \\
\text { structure }\end{array}$} & \multirow[b]{2}{*}{ WT } & \multirow[b]{2}{*}{ Het } & \multirow[b]{2}{*}{ KO } & \multicolumn{3}{|c|}{ Genotype } & \multicolumn{3}{|l|}{ Cohort } & \multicolumn{3}{|c|}{$\begin{array}{l}\text { Genotype } \times \\
\text { cohort }\end{array}$} & \multicolumn{3}{|c|}{$\begin{array}{l}\text { Pairwise } \\
\text { comparisons }\end{array}$} \\
\hline & & & & & & $F$ & $\begin{array}{l}p \\
\text { value }\end{array}$ & Power & $F$ & $\begin{array}{l}p \\
\text { value }\end{array}$ & Power & $F$ & $\begin{array}{l}p \\
\text { value }\end{array}$ & Power & $\begin{array}{l}\text { WT vs } \\
\text { Het }\end{array}$ & $\begin{array}{l}\text { WT vs } \\
\text { KO }\end{array}$ & $\begin{array}{l}\text { Het vs } \\
\text { KO }\end{array}$ \\
\hline $\begin{array}{l}\text { Paw misplacements } \\
\text { (large, all mice) }\end{array}$ & 2WANOVA & Nonnormal & $0.47 \pm 0.14$ & $0.56 \pm 0.16$ & $1.06 \pm 0.14$ & 4.197 & 0.021 & 0.712 & 0.137 & 0.713 & 0.065 & 1.662 & 0.200 & 0.334 & 0.693 & 0.010 & 0.026 \\
\hline $\begin{array}{l}\text { Paw misplacements } \\
\text { (medium, all mice) }\end{array}$ & 2WANOVA & Normal & $1.71 \pm 0.29$ & $1.5 \pm 0.26$ & $2.38 \pm 0.41$ & 1.762 & 0.182 & 0.352 & 0.525 & 0.472 & 0.110 & 0.055 & 0.947 & 0.058 & - & - & - \\
\hline $\begin{array}{l}\text { Paw misplacements } \\
\text { (small, all mice) }\end{array}$ & 2WANOVA & Nonnormal & $1.44 \pm 0.13$ & $2.48 \pm 0.47$ & $1.78 \pm 0.18$ & 3.330 & 0.044 & 0.605 & 3.700 & 0.060 & 0.471 & 0.760 & 0.473 & 0.172 & 0.015 & 0.462 & 0.082 \\
\hline $\begin{array}{l}\text { Paw misplacements } \\
\text { (large, crossing mice) }\end{array}$ & 2WANOVA & Nonnormal & $0.52 \pm 0.17$ & $0.6 \pm 0.17$ & $1.07 \pm 0.14$ & 3.194 & 0.049 & 0.585 & 0.210 & 0.649 & 0.073 & 1.333 & 0.273 & 0.275 & 0.937 & 0.053 & 0.119 \\
\hline $\begin{array}{l}\text { Paw misplacements (medium, } \\
\text { crossing mice) }\end{array}$ & 2WANOVA & Nonnormal & $1.67 \pm 0.33$ & $1.37 \pm 0.27$ & $2.33 \pm 0.53$ & 1.343 & 0.271 & 0.276 & 0.851 & 0.361 & 0.148 & 0.027 & 0.973 & 0.054 & - & - & - \\
\hline $\begin{array}{l}\text { Paw misplacements (small, } \\
\text { crossing mice) }\end{array}$ & 2WANOVA & Nonnormal & $1.79 \pm 0.23$ & $2.77 \pm 0.53$ & $2.81 \pm 0.64$ & 1.189 & 0.327 & 0.227 & 2.465 & 0.134 & 0.318 & 0.102 & 0.904 & 0.063 & - & - & - \\
\hline $\begin{array}{l}\text { Time to cross (large, fully } \\
\text { crossing) }\end{array}$ & 2WANOVA & Nonnormal & $10.05 \pm 1.3$ & $9.11 \pm 1.91$ & $7.17 \pm 1.15$ & 0.911 & 0.409 & 0.199 & 3.043 & 0.087 & 0.402 & 1.213 & 0.306 & 0.253 & - & - & - \\
\hline $\begin{array}{l}\text { Time to cross (medium, fully } \\
\text { crossing) }\end{array}$ & 2WANOVA & Nonnormal & $28.54 \pm 5.32$ & $16.62 \pm 3.28$ & $18.14 \pm 4.47$ & 1.643 & 0.204 & 0.330 & 2.898 & 0.095 & 0.386 & 2.415 & 0.100 & 0.464 & - & - & - \\
\hline $\begin{array}{l}\text { Time to cross (small, fully } \\
\text { crossing) }\end{array}$ & 2WANOVA & Normal & $54.56 \pm 7.16$ & $44.74 \pm 5.25$ & $22.43 \pm 6$ & 4.119 & 0.030 & 0.667 & 0.037 & 0.850 & 0.054 & 2.660 & 0.092 & 0.473 & 0.479 & 0.030 & 0.169 \\
\hline Time to cross (large, all mice) & 2WANOVA & Nonnormal & $15.75 \pm 3.07$ & $20.75 \pm 6.73$ & $8.5 \pm 2.21$ & 4.443 & 0.027 & 0.370 & 0.327 & 0.574 & 0.094 & 0.427 & 0.659 & 0.070 & 0.015 & 0.033 & 0.820 \\
\hline $\begin{array}{l}\text { Time to cross (medium, all } \\
\text { mice) }\end{array}$ & 2WANOVA & Nonnormal & $46.34 \pm 8.74$ & $36.11 \pm 8.58$ & $34.61 \pm 8.93$ & 1.157 & 0.337 & 0.109 & 1.444 & 0.245 & 0.682 & 0.472 & 0.632 & 0.218 & - & - & - \\
\hline Time to cross (small, all mice) & 2WANOVA & Normal & $99.92 \pm 4.67$ & $89.98 \pm 7.71$ & $111.65 \pm 4.61$ & 3.540 & 0.051 & 0.606 & 0.524 & 0.478 & 0.073 & 3.236 & 0.063 & 0.158 & 0.017 & 0.428 & 0.220 \\
\hline \multicolumn{18}{|l|}{ Motor reflexes } \\
\hline & & & & & & \multicolumn{3}{|c|}{ Genotype } & \multicolumn{3}{|l|}{ Cohort } & \multicolumn{3}{|c|}{$\begin{array}{l}\text { Genotype } \times \\
\text { cohort }\end{array}$} & \multicolumn{3}{|c|}{$\begin{array}{l}\text { Pairwise } \\
\text { comparisons }\end{array}$} \\
\hline & Test & $\begin{array}{l}\text { Data } \\
\text { structure }\end{array}$ & WT & Het & KO & $F$ & $\begin{array}{l}p \\
\text { value }\end{array}$ & Power & $F$ & $\begin{array}{l}p \\
\text { value }\end{array}$ & Power & $F$ & $\begin{array}{l}p \\
\text { value }\end{array}$ & Power & $\begin{array}{l}\text { WT vs } \\
\text { Het }\end{array}$ & $\begin{array}{l}\text { WT vs } \\
\text { KO }\end{array}$ & $\begin{array}{l}\text { Het vs } \\
\text { KO }\end{array}$ \\
\hline Righting reflex & 2WANOVA & Nonnormal & $0.05 \pm 0.05$ & $0 \pm 0$ & $0 \pm 0$ & 0.721 & 0.491 & 0.165 & 0.727 & 0.398 & 0.133 & 0.721 & 0.491 & 0.165 & - & - & - \\
\hline Hindlimb placing, score & 2WANOVA & Nonnormal & $5.57 \pm 0.24$ & $5.26 \pm 0.34$ & $4.21 \pm 0.57$ & 2.778 & 0.072 & 0.524 & 0.093 & 0.762 & 0.060 & 0.117 & 0.890 & 0.067 & 0.618 & 0.029 & 0.086 \\
\hline $\begin{array}{l}\text { Hindlimb placing, latency } \\
\text { to climb }\end{array}$ & 2WANOVA & Nonnormal & $8.29 \pm 1.61$ & $7.21 \pm 1.66$ & $10.96 \pm 2.57$ & 0.836 & 0.439 & 0.186 & 0.333 & 0.567 & 0.087 & 0.117 & 0.890 & 0.067 & - & - & - \\
\hline $\begin{array}{l}\text { Hindlimb placing, failed } \\
\text { attempts }\end{array}$ & 2WANOVA & Nonnormal & $0.21 \pm 0.12$ & $0.36 \pm 0.17$ & $0.89 \pm 0.28$ & 2.778 & 0.072 & 0.524 & 0.093 & 0.762 & 0.060 & 0.117 & 0.890 & 0.067 & 0.618 & 0.029 & 0.086 \\
\hline $\begin{array}{l}\text { Inverted screen, latency } \\
\text { to fall }\end{array}$ & 2WANOVA & Nonnormal & $33.78 \pm 5.09$ & $37 \pm 4.72$ & $9 \pm 3.13$ & 11.464 & 0.000 & 0.991 & 0.701 & 0.406 & 0.130 & 0.645 & 0.529 & 0.152 & 0.522 & 0.000 & 0.000 \\
\hline Hanging, score & 2WANOVA & Nonnormal & $6.26 \pm 0.18$ & $6 \pm 0.25$ & $4.84 \pm 0.27$ & 10.223 & 0.000 & 0.982 & 2.691 & 0.107 & 0.363 & 0.834 & 0.440 & 0.185 & 0.486 & 0.000 & 0.001 \\
\hline Hanging, latency to fall & 2WANOVA & Nonnormal & $25.44 \pm 2.27$ & $23.66 \pm 2.66$ & $8.48 \pm 1.05$ & 18.838 & 0.000 & 1.000 & 0.269 & 0.606 & 0.080 & 0.816 & 0.448 & 0.182 & 0.643 & 0.000 & 0.000 \\
\hline \multicolumn{18}{|l|}{ Grip strength } \\
\hline Latency & Test & $\begin{array}{l}\text { Data } \\
\text { structure }\end{array}$ & & & & $F$ & $\begin{array}{l}p \\
\text { value }\end{array}$ & Power & $\begin{array}{l}\text { WT vs } \\
\text { Het }\end{array}$ & $\begin{array}{l}\text { WT vs } \\
\text { KO }\end{array}$ & $\begin{array}{l}\text { Het vs } \\
\text { KO }\end{array}$ & & & & & & \\
\hline - Session & rMeasures & Sph.viol & & & & 3.520 & 0.033 & 0.644 & - & - & - & & & & & & \\
\hline - Session $\times$ gen. & rMeasures & Sph.viol & & & & 1.971 & 0.105 & 0.575 & - & - & - & & & & & & \\
\hline - Genotype & rMeasures & Sph.viol & & & & 0.324 & 0.725 & 0.099 & - & - & - & & & & & & \\
\hline - Cohort & rMeasures & Sph.viol & & & & 47.402 & 0.000 & 1.000 & - & - & - & & & & & & \\
\hline - Session $\times$ gen. $\times$ coh & rMeasures & Sph.viol & & & & 2.687 & 0.035 & 0.729 & - & - & - & & & & & & \\
\hline \multirow[t]{2}{*}{ - Gen. $\times$ coh. } & rMeasures & Sph.viol & & & & 1.044 & 0.359 & 0.223 & - & - & - & & & & & & \\
\hline & & & & & & \multicolumn{3}{|c|}{ Genotype } & \multicolumn{3}{|l|}{ Cohort } & \multicolumn{3}{|c|}{$\begin{array}{l}\text { Genotype } \times \\
\text { cohort }\end{array}$} & \multicolumn{3}{|c|}{$\begin{array}{l}\text { Pairwise } \\
\text { comparisons }\end{array}$} \\
\hline Individual trials & Test & $\begin{array}{l}\text { Data } \\
\text { structure }\end{array}$ & WT & Het & $\mathrm{KO}$ & $F$ & $\begin{array}{l}p \\
\text { value }\end{array}$ & Power & $F$ & $\begin{array}{l}p \\
\text { value }\end{array}$ & Power & $F$ & $\begin{array}{l}p \\
\text { value }\end{array}$ & Power & $\begin{array}{l}\text { WT vs } \\
\text { Het }\end{array}$ & $\begin{array}{l}\text { WT vs } \\
\text { KO }\end{array}$ & $\begin{array}{l}\text { Het vs } \\
\mathrm{KO}\end{array}$ \\
\hline Session 1 & 2WANOVA & Normal & $0.94 \pm 0.05$ & $0.99 \pm 0.06$ & $1.03 \pm 0.06$ & 0.502 & 0.608 & 0.128 & 25.973 & 0.000 & 0.999 & 1.564 & 0.219 & 0.317 & - & - & - \\
\hline Session 2 & 2WANOVA & Normal & $0.86 \pm 0.07$ & $0.85 \pm 0.06$ & $1.01 \pm 0.05$ & 2.222 & 0.119 & 0.433 & 36.967 & 0.000 & 1.000 & 1.631 & 0.206 & 0.329 & - & - & - \\
\hline Session 3 & 2WANOVA & Normal & $0.91 \pm 0.06$ & $0.92 \pm 0.06$ & $0.89 \pm 0.06$ & 0.320 & 0.728 & 0.098 & 23.585 & 0.000 & 0.997 & 1.883 & 0.163 & 0.374 & - & - & - \\
\hline Mean strength & 2WANOVA & Normal & $0.9 \pm 0.05$ & $0.92 \pm 0.05$ & $0.98 \pm 0.04$ & 0.324 & 0.725 & 0.099 & 47.402 & 0.000 & 1.000 & 1.044 & 0.359 & 0.223 & - & - & - \\
\hline Highest score & 2WANOVA & Nonnormal & $1.08 \pm 0.05$ & $1.07 \pm 0.05$ & $1.13 \pm 0.05$ & 0.243 & 0.785 & 0.086 & 26.793 & 0.000 & 0.999 & 0.821 & 0.446 & 0.183 & - & - & - \\
\hline
\end{tabular}

WT, wild-type mice; Het, heterozygous mice; KO, homozygous knock-out mice. Group values are reported as mean \pm SEM. Bold font indicates significant results $(p<0.05)$. Individual results and statistical analyses for cohorts 1 and 2 are available in Extended Data Table 6-1. 2wANOVA: 2-way ANOVA, rMeasures: repeated measures, Sph.viol: sphericity violated, gen: genotype, coh: cohort.

are expected to spend more time investigating a novel object than a familiar object, and this is what was observed for wild-type and heterozygous mice (Fig. 6A, left panel). However, in homozygous mice, results were difficult to interpret due to strikingly reduced object interactions (Fig. 6A, left and middle panels). Homozygous mice spent most of both of the test sessions (the first involving familiarization with identical objects and the second involving interaction with one familiar and one novel object) away from both objects, spending excessive time in the corners of the open field as shown on heatmaps (Extended Data Fig. 6-1A) and demon- strating longer latency to explore any of the objects (Fig. 6A, right panel).

Object avoidance was further confirmed in multiple independent tests, including the marble burying, a test used to assess stereotypic behavior and/or anxiety. In this paradigm, 20 marbles were spread across the cage floor in a $4 \times 5$ pattern, leaving little space for the mice to move around the marbles. While both wild-type and Shank3 ${ }^{\Delta 4-22}$ heterozygous mice quickly buried most of the marbles as is typical, Shank $3^{\Delta 4-22}$ homozygous mice left the marbles almost completely undisturbed for the whole 15-min duration of the test (Fig. 6B; Extended Data Fig. 6-1B). 
A

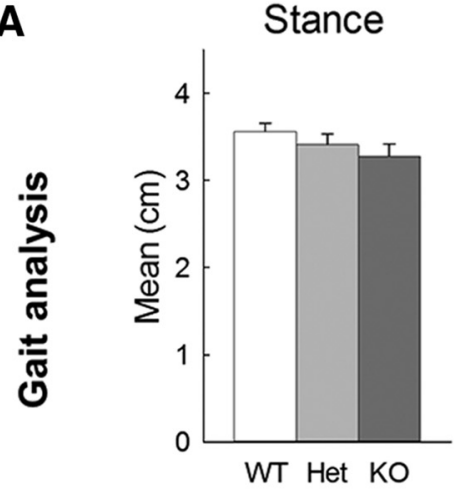

B

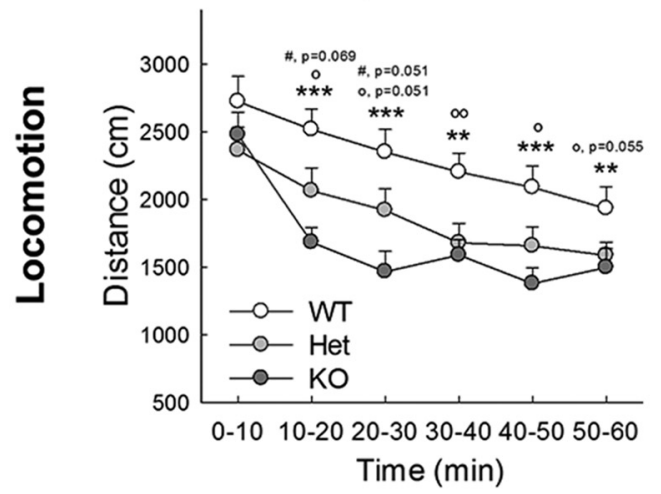

Stride

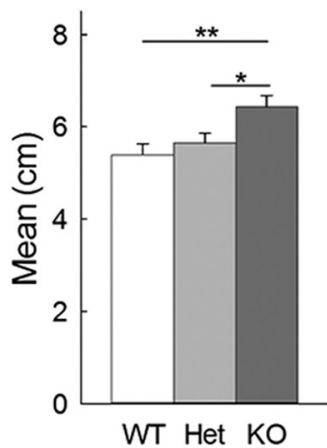

Sway

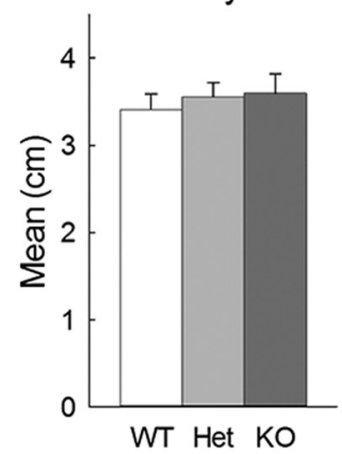

Rotarod

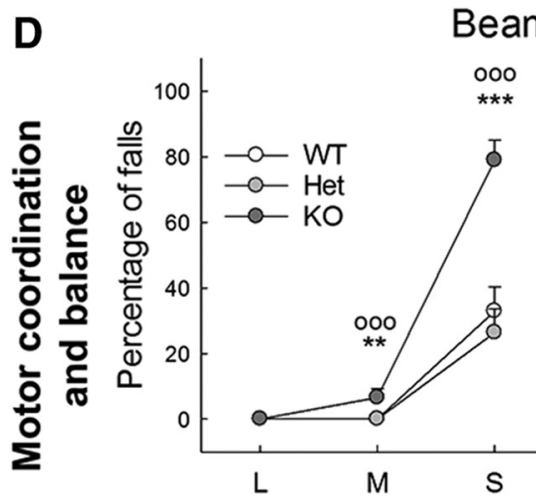

C

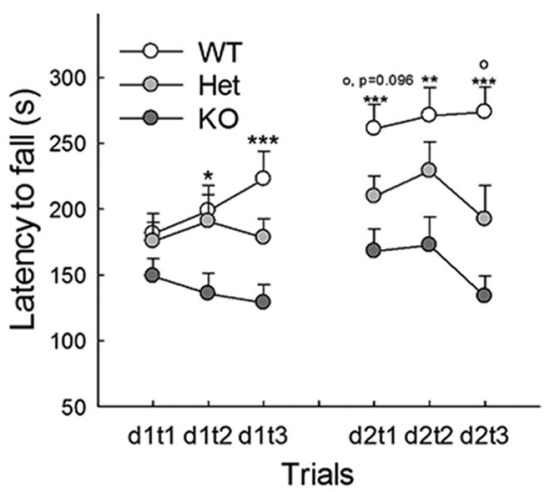

E

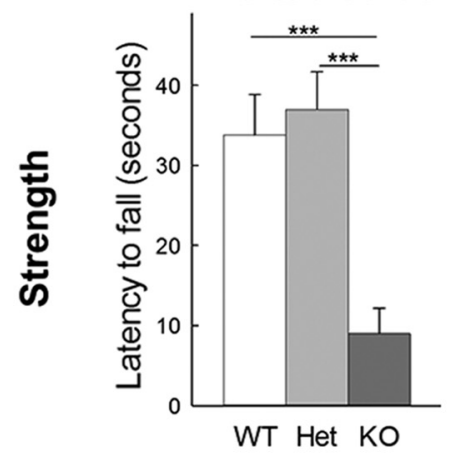

Hanging

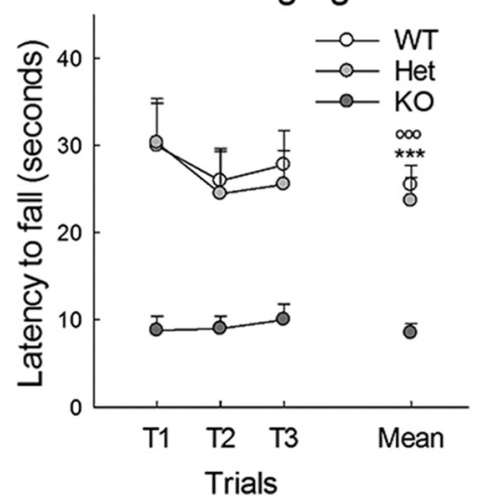

Figure 3. Impaired motor performances in in Shank3 $3^{44-22}$-deficient mice. A, Average stance, stride, and sway. Gait analysis showed an increase stride length in Shank $3^{\Delta 4-22}$ homozygous mice. $\boldsymbol{B}$, Distance traveled during a $60-$ min session in an open field. 


\section{continued}

Spontaneous locomotor activity in the open field was reduced in Shank3 ${ }^{\Delta 4-22}$ homozygous mice relative to other genotypes. $\boldsymbol{C}$, Latency to fall over six trials (three trials per day for two consecutive days) in the accelerating rotarod task. Motor learning on the accelerating rotarod was deficient in Shank $3^{\Delta 4-22}$ homozygous mice compared to wild-type animals as they failed to improve over time. Heterozygous mice had an intermediate phenotype. $\boldsymbol{D}$, Percentage of falls and distance crossed during the beam walking test. While not different on the large (L, 1 inch) and medium (M, 1/2 inch) beams, Shank $3^{\Delta 4-22}$ homozygous mice were strongly impaired in the small (S, $1 / 4$ inch) beam walking test, as shown by a significant increase of the number of falls and a decrease of the distance crossed. $\boldsymbol{E}$, Strength and endurance measured in the inverted screen and hanging tests. Endurance strength was significantly impaired in Shank $3^{\Delta 4-22}$ homozygous mice as they exhibited significantly shorter latency to fall in both the inverted screen and hanging tests. Additional results of motor tests (hindlimb placing and grip strength) are available in Extended Data Figure 3-1. WT, wild-type mice; Het, heterozygous mice; KO, homozygous knock-out mice. *: WT versus KO; o: WT versus Het, \#: Het versus KO. $* p<0.05, * * p<0.1, * * * p<0.001$

Consistent with these result, a significant decrease of the time spent exploring objects in the four-object exploration test was observed in the Shank $3^{\Delta 4-22}$ homozygous mice as compared to their littermate (Fig. 6C).

During assessment of nest building, nests build by Shank $3^{\Delta 4-22}$ homozygous mice were significantly less elaborate than nests built by wild-type or heterozygous mice, with some homozygotes leaving the nesting material completely untouched (Fig. 6D; Extended Data Fig. 6-1C). Note that, in an attempt to reduce stress and improve breeding rates, dams used to produce the cohorts described here were provided with plastic huts in their home cage. Interestingly, while most of the wild-type dams (seven out of ten) chose to build their nest inside the huts, only a single Shank $3^{\Delta 4-22}$ heterozygous dam out of 20 used the hut to establish their nests (wild-type vs heterozygotes: $\left.t_{(28)}=-5.085, p<0.001\right)$. Additionally, three of the Shank $3^{\Delta 4-22}$ heterozygous dams did not build a nest until after the birth.

Object avoidance might also explain the reduction of the total time of direct interactions (grabbing, touching, biting, or climbing) with the applicator used to present the different scents during the olfactory habituation and dishabituation test in Shank3 $3^{\Delta 4-22}$ homozygous mice, compared to their wild-type and heterozygous littermates (Fig. $6 E)$.

\section{Hyper-reactivity and escape behaviors in Shank $3^{\Delta 4-}$ 22-deficient mice}

Unusual hyper-reactivity was observed in Shank $3^{\Delta 4-22}$ homozygous mice during handling and confirmed in several behavioral tests (Table 10). This hyper-reactivity was characterized by a higher score in the touch escape test (Fig. 7A, left panel), a lower score (reflecting a higher tendency to struggle in response to sequential handling) in the positional passivity (Fig. 7A, middle panel), and a shorter latency to move from the beam during the catalepsy test (Fig. 7A, right panel). As in newborn mice, a shorter latency to turn was seen for Shank $3^{\Delta 4-22}$ homozygous mice in the negative geotaxis test (Fig. 7B, left panel). Similarly, in the beam walking test, the latency to start crossing on the smallest beam was shorter in Shank $3^{\Delta 4-22}$ homozygous mice (Fig. $7 B$, right panel) but often led to a premature fall (Fig. 3D).

Escape attempts were observed in several tests and high-wall enclosures had to be built around testing cages to prevent successful attempts. Escape behaviors were scored in three different home cage tests. During the habituation portion of the buried food test (where no objects were visible at the surface of the cage bedding), no escape behavior nor genotype differences were observed (Fig. 7C, left panel). However, when the mice were tested in the same cages during the four-object interaction test both the number of escape attempts and the percentage of mice engaged in this behavior increased and significant genotype differences were observed (Fig. $7 C$, middle panel). This behavior was even more marked in the marble burying test (Fig. $7 \mathrm{C}$, right panel), during which $94 \%$ of heterozygous mice and $100 \%$ of homozygous mice tried to escape. This indicated that the escape behavior is elicited by the presence of unfamiliar objects in the testing cage.

\section{Repetitive behaviors, stereotypies, and inflexibly in Shank $3^{\Delta-22}$-deficient mice}

Repetitive and restricted behaviors are one of the core features of ASD. Therefore, during all of the behavioral tests, mice were also carefully monitored for stereotypies, as well as perseverative and repetitive behaviors. Detailed results are reported in Table 11.

While no genotype difference was observed in the number of spontaneous grooming bouts observed during the 10 first minutes of the open field test, Shank $3^{\Delta 4-22}$ homozygous mice engaged in longer episodes of selfgrooming, as shown by a significant increase in the cumulative time spent grooming all body regions when compared to their wild-type and heterozygous littermates. However, skin lesions were frequently observed in older mice (over eight-month-old) of all three genotypes without obvious genotype effect. Significantly more rotations were also observed in Shank $3^{\Delta 4-22}$ homozygous animals as well as a trend toward an increase of head twitching/ shaking in both Shank $3^{\Delta 4-22}$ heterozygous and homozygous mice, as compared to their wild-type littermates (Fig. $8 A)$.

Object preferences, exploration patterns and frequency of repetitive contacts with novel objects were evaluated in the repetitive novel object contact task. Although the cumulative time spend interacting with the objects was decreased in Shank $3^{\Delta 4-22}$ homozygous mice (Fig. $6 C$ ), this test failed to display genotype difference in either the total number of interactions, the preference for any specific objects or the preference for any specific preferred sequence of threeobject or four-object explorations (Fig. 8B). 
Table 7. Detailed results and statistical analyses related to the sensory profile

\begin{tabular}{|c|c|c|c|c|c|c|c|c|c|c|c|c|c|c|c|c|c|}
\hline \multicolumn{18}{|c|}{ Reflexes and reactions to simple stimuli } \\
\hline & \multirow[b]{2}{*}{ Test } & \multirow[b]{2}{*}{$\begin{array}{l}\text { Data } \\
\text { structure }\end{array}$} & \multirow[b]{2}{*}{ WT } & \multirow[b]{2}{*}{ Het } & \multirow[b]{2}{*}{$\mathrm{KO}$} & \multicolumn{2}{|c|}{ Genotype } & \multirow[b]{2}{*}{ Power } & \multicolumn{2}{|l|}{ Cohort } & \multirow[b]{2}{*}{ Power } & \multicolumn{3}{|c|}{$\begin{array}{l}\text { Genotype } \times \\
\text { cohort }\end{array}$} & $\begin{array}{l}\text { Pairwis } \\
\text { compar }\end{array}$ & $\begin{array}{l}\text { risons } \\
\text { riso }\end{array}$ & \\
\hline & & & & & & $F$ & $\begin{array}{l}p \\
\text { value }\end{array}$ & & $F$ & $\begin{array}{l}p \\
\text { value }\end{array}$ & & $F$ & $\begin{array}{l}p \\
\text { value }\end{array}$ & Power & $\begin{array}{l}\text { WT vs } \\
\text { Het }\end{array}$ & $\begin{array}{l}\text { WT vs } \\
\text { KO }\end{array}$ & $\begin{array}{l}\text { Het vs } \\
\text { KO }\end{array}$ \\
\hline Pinna reflex & 2WANOVA & Nonnormal & $0.89 \pm 0.07$ & $0.68 \pm 0.1$ & $0.73 \pm 0.1$ & 1.790 & 0.1773 & 0.357 & 13.988 & 0.000 & 0.956 & 1.155 & 0.323 & 0.243 & - & - & - \\
\hline Cornel reflex & 2WANOVA & Nonnormal & $1.05 \pm 0.05$ & $0.94 \pm 0.05$ & $1.05 \pm 0.05$ & 1.518 & 0.2289 & 0.308 & 0.389 & 0.536 & 0.094 & 1.518 & 0.229 & 0.308 & - & - & - \\
\hline Toe pinch retraction & 2WANOVA & Nonnormal & $2.05 \pm 0.37$ & $2.36 \pm 0.33$ & $2.26 \pm 0.43$ & 0.144 & 0.8659 & 0.071 & 1.073 & 0.305 & 0.174 & 0.072 & 0.931 & 0.060 & - & - & - \\
\hline Preyer reflex & 2WANOVA & Nonnormal & $1.47 \pm 0.15$ & $1.36 \pm 0.13$ & $1.42 \pm 0.17$ & 0.135 & 0.8740 & 0.070 & 22.250 & 0.000 & 0.996 & 1.478 & 0.238 & 0.301 & - & - & - \\
\hline Visual placing & NA & NA & $9 \pm 0$ & $9 \pm 0$ & $9 \pm 0$ & - & - & & - & - & & - & - & & - & - & - \\
\hline Tail flick & & & & & & & & & & & & & & & & & \\
\hline Latency & Test & $\begin{array}{l}\text { Data } \\
\text { structure }\end{array}$ & & & & $F$ & $\begin{array}{l}p \\
\text { value }\end{array}$ & Power & $\begin{array}{l}\text { WT vs } \\
\text { Het }\end{array}$ & $\begin{array}{l}\text { WT vs } \\
\text { KO }\end{array}$ & $\begin{array}{l}\text { Het vs } \\
\text { KO }\end{array}$ & & & & & & \\
\hline - Trial & rMeasures & Sph.viol & & & & 3.081 & 0.0500 & 0.583 & - & - & - & & & & & & \\
\hline - Trial $\times$ gen & rMeasures & Sph.viol & & & & 0.169 & 0.9535 & 0.085 & - & - & - & & & & & & \\
\hline - Genotype & rMeasures & Sph.viol & & & & 1.118 & 0.3347 & 0.236 & - & - & - & & & & & & \\
\hline - Cohort & rMeasures & Sph.viol & & & & 83.467 & 0.0000 & 1.000 & - & - & - & & & & & & \\
\hline - Trial $\times$ gen. $\times$ coh & rMeasures & Sph.viol & & & & 0.489 & 0.7438 & 0.162 & - & - & - & & & & & & \\
\hline - Gen. $\times$ coh. & rMeasures & Sph.viol & & & & 2.162 & 0.1255 & 0.422 & - & - & - & & & & & & \\
\hline & & & & & & Genotyp & & & Cohort & & & $\begin{array}{l}\text { Genoty } \\
\text { cohort }\end{array}$ & type $\times$ & & $\begin{array}{l}\text { Pairwis } \\
\text { compar }\end{array}$ & risons & \\
\hline Individual trials & Test & $\begin{array}{l}\text { Data } \\
\text { structure }\end{array}$ & WT & Het & KO & $F$ & $\begin{array}{l}p \\
\text { value }\end{array}$ & Power & $F$ & $\begin{array}{l}p \\
\text { value }\end{array}$ & Power & $F$ & $\begin{array}{l}p \\
\text { value }\end{array}$ & Power & $\begin{array}{l}\text { WT vs } \\
\text { Het }\end{array}$ & $\begin{array}{l}\text { WT vs } \\
\text { KO }\end{array}$ & $\begin{array}{l}\text { Het vs } \\
\text { KO }\end{array}$ \\
\hline Latency trial 1 (s) & 2WANOVA & Normal & $11.39 \pm 0.97$ & $11.32 \pm 0.78$ & $10.28 \pm 0.82$ & 0.356 & 0.7023 & 0.104 & 13.680 & 0.001 & 0.952 & 0.145 & 0.865 & 0.071 & - & - & - \\
\hline Latency trial 2 (s) & 2WANOVA & Normal & $10.99 \pm 1.08$ & $9.67 \pm 0.98$ & $8.86 \pm 0.82$ & 0.961 & 0.3892 & 0.208 & 36.614 & 0.000 & 1.000 & 2.286 & 0.112 & 0.444 & - & - & - \\
\hline Latency trial 3 (s) & 2WANOVA & Normal & $11.05 \pm 1.23$ & $10.06 \pm 1.01$ & $9.29 \pm 0.94$ & 0.526 & 0.5942 & 0.132 & 91.329 & 0.000 & 1.000 & 1.578 & 0.216 & 0.319 & - & - & - \\
\hline Shortest latency (s) & 2WANOVA & Normal & $8.03 \pm 0.82$ & $7.99 \pm 0.87$ & $7.47 \pm 0.78$ & 0.064 & 0.9379 & 0.059 & 42.964 & 0.000 & 1.000 & 1.039 & 0.361 & 0.222 & - & - & - \\
\hline Longest latency (s) & 2WANOVA & Nonnormal & $14.17 \pm 0.94$ & $12.82 \pm 0.75$ & $11.91 \pm 0.72$ & 2.213 & 0.1198 & 0.431 & 64.815 & 0.000 & 1.000 & 1.659 & 0.200 & 0.334 & - & - & - \\
\hline Mean latency (s) & 2WANOVA & Nonnormal & $11.14 \pm 0.88$ & $10.35 \pm 0.78$ & $9.48 \pm 0.72$ & 1.118 & 0.3347 & 0.236 & 83.467 & 0.000 & 1.000 & 2.162 & 0.126 & 0.422 & - & - & - \\
\hline Startle response & & & & & & & & & & & & & & & & & \\
\hline & & & & & & Genotyp & & & Cohort & & & $\begin{array}{l}\text { Genoty } \\
\text { cohort }\end{array}$ & ype $\times$ & & $\begin{array}{l}\text { Pairwis } \\
\text { compar }\end{array}$ & $\begin{array}{l}\text { risons } \\
\text { ris }\end{array}$ & \\
\hline & Test & $\begin{array}{l}\text { Data } \\
\text { structure }\end{array}$ & WT & Het & KO & $F$ & $\begin{array}{l}p \\
\text { value }\end{array}$ & Power & $F$ & $\begin{array}{l}p \\
\text { value }\end{array}$ & Power & $F$ & $\begin{array}{l}p \\
\text { value }\end{array}$ & Power & $\begin{array}{l}\text { WT vs } \\
\text { Het }\end{array}$ & $\begin{array}{l}\text { WT vs } \\
\text { KO }\end{array}$ & $\begin{array}{l}\text { Het vs } \\
\text { KO }\end{array}$ \\
\hline Startle $74 \mathrm{~dB}$ & 2WANOVA & Nonnormal & $179.96 \pm 20.24$ & $165.57 \pm 17.22$ & $158.01 \pm 10.36$ & 2.376 & 0.1077 & 0.448 & 9.123 & 0.005 & 0.836 & 2.255 & 0.120 & 0.428 & - & - & - \\
\hline Startle $78 \mathrm{~dB}$ & 2WANOVA & Nonnormal & $183.48 \pm 22$ & $157.4 \pm 16.93$ & $160.69 \pm 14.89$ & 3.181 & 0.0538 & 0.571 & 5.779 & 0.022 & 0.647 & 3.673 & 0.036 & 0.638 & 0.046 & 0.025 & 0.770 \\
\hline Startle $82 \mathrm{~dB}$ & 2WANOVA & Nonnormal & $197.45 \pm 26.03$ & $160.09 \pm 14.07$ & $175.58 \pm 13.87$ & 3.254 & 0.0500 & 0.582 & 3.939 & 0.055 & 0.488 & 2.621 & 0.087 & 0.487 & 0.019 & 0.063 & 0.591 \\
\hline Startle $86 \mathrm{~dB}$ & 2WANOVA & Nonnormal & $246.33 \pm 35.36$ & $162.3 \pm 15.61$ & $176.15 \pm 12.88$ & 3.255 & 0.0500 & 0.582 & 0.082 & 0.777 & 0.059 & 0.271 & 0.764 & 0.089 & 0.024 & 0.042 & 0.812 \\
\hline Startle $92 \mathrm{~dB}$ & 2WANOVA & Nonnormal & $257.25 \pm 40.4$ & $192.63 \pm 19.03$ & $201.41 \pm 23.01$ & 2.153 & 0.1313 & 0.411 & 0.039 & 0.845 & 0.054 & 1.323 & 0.279 & 0.267 & - & - & - \\
\hline Startle response & Test & $\begin{array}{l}\text { Data } \\
\text { structure }\end{array}$ & & & & $F$ & $\begin{array}{l}p \\
\text { value }\end{array}$ & Power & $\begin{array}{l}\text { WT vs } \\
\text { Het }\end{array}$ & $\begin{array}{l}\text { WT vs } \\
\text { KO }\end{array}$ & $\begin{array}{l}\text { Het vs } \\
\text { KO }\end{array}$ & & & & & & \\
\hline - Sound intensity & rMeasures & Sph.ass & & & & 2.900 & 0.0510 & 0.605 & - & - & - & & & & & & \\
\hline - Sound intensity $\times$ gen. & rMeasures & Sph.ass & & & & 0.642 & 0.6620 & 0.219 & - & - & - & & & & & & \\
\hline - Genotype & rMeasures & Sph.ass & & & & 3.649 & 0.0364 & 0.635 & 0.022 & 0.024 & 0.989 & & & & & & \\
\hline - Cohort & rMeasures & Sph.ass & & & & 1.842 & 0.1835 & 0.262 & - & - & - & & & & & & \\
\hline - Sound intensity $\times$ gen. $\times$ coh. & rMeasures & Sph.ass & & & & 0.822 & 0.5335 & 0.276 & - & - & - & & & & & & \\
\hline - Gen. $\times$ coh. & rMeasures & Sph.ass & & & & 1.922 & 0.1614 & 0.372 & - & - & - & & & & & & \\
\hline & & & & & & Genotyp & & & Cohort & & & $\begin{array}{l}\text { Genoty } \\
\text { cohort }\end{array}$ & type $\times$ & & $\begin{array}{l}\text { Pairwis } \\
\text { compar }\end{array}$ & $\begin{array}{l}\text { risons } \\
\text { riso }\end{array}$ & \\
\hline & Test & $\begin{array}{l}\text { Data } \\
\text { structure }\end{array}$ & WT & Het & KO & $F$ & $\begin{array}{l}p \\
\text { value }\end{array}$ & Power & $F$ & $\begin{array}{l}p \\
\text { value }\end{array}$ & Power & $F$ & $\begin{array}{l}p \\
\text { value }\end{array}$ & Power & $\begin{array}{l}\text { WT vs } \\
\text { Het }\end{array}$ & $\begin{array}{l}\text { WT vs } \\
\text { KO }\end{array}$ & $\begin{array}{l}\text { Het } \\
\text { vs KO }\end{array}$ \\
\hline Startle $74 \mathrm{~dB}$ norm. to weight & 2WANOVA & Nonnormal & $7.01 \pm 0.82$ & $6.18 \pm 0.64$ & $6.17 \pm 0.32$ & 2.843 & 0.0718 & 0.522 & 5.749 & 0.022 & 0.645 & 2.780 & 0.076 & 0.512 & 0.045 & 0.042 & 0.959 \\
\hline Startle $78 \mathrm{~dB}$ norm. to weight & 2WANOVA & Nonnormal & $7.19 \pm 0.89$ & $5.86 \pm 0.64$ & $6.35 \pm 0.58$ & 3.051 & 0.0601 & 0.553 & 2.972 & 0.094 & 0.389 & 3.611 & 0.038 & 0.630 & 0.038 & 0.036 & 0.964 \\
\hline Startle $82 \mathrm{~dB}$ norm. to weight & 2WANOVA & Nonnormal & $7.74 \pm 1.06$ & $5.89 \pm 0.41$ & $6.98 \pm 0.63$ & 3.378 & 0.0456 & 0.599 & 1.700 & 0.201 & 0.245 & 2.365 & 0.109 & 0.446 & 0.015 & 0.089 & 0.409 \\
\hline Startle $86 \mathrm{~dB}$ norm. to weight & 2WANOVA & Nonnormal & $9.86 \pm 1.66$ & $6 \pm 0.49$ & $7.03 \pm 0.64$ & 2.888 & 0.0690 & 0.529 & 0.030 & 0.864 & 0.053 & 0.268 & 0.766 & 0.089 & 0.028 & 0.070 & 0.677 \\
\hline Startle $92 \mathrm{~dB}$ norm. to weight & 2WANOVA & Nonnormal & $10.04 \pm 1.77$ & $7.09 \pm 0.58$ & $8.18 \pm 1.15$ & 1.758 & 0.1873 & 0.343 & 0.323 & 0.573 & 0.086 & 1.291 & 0.288 & 0.261 & - & - & - \\
\hline $\begin{array}{l}\text { Startle response normalized } \\
\text { to weight }\end{array}$ & Test & $\begin{array}{l}\text { Data } \\
\text { structure }\end{array}$ & & & & $F$ & $\begin{array}{l}p \\
\text { value }\end{array}$ & Power & $\begin{array}{l}\text { WT vs } \\
\text { Het }\end{array}$ & $\begin{array}{l}\text { WT vs } \\
\text { KO }\end{array}$ & $\begin{array}{l}\text { Het } \\
\text { vs KO }\end{array}$ & & & & & & \\
\hline - Sound intensity & rMeasures & Sph.ass & & & & 2.506 & 0.0449 & 0.248 & - & - & - & & & & & & \\
\hline - Sound intensity $\times$ gen. & rMeasures & Sph.ass & & & & 0.580 & 0.7933 & 0.098 & - & - & - & & & & & & \\
\hline - Genotype & rMeasures & Sph.ass & & & & 3.338 & 0.0471 & 0.593 & 0.127 & 0.407 & 0.783 & & & & & & \\
\hline - Cohort & rMeasures & Sph.ass & & & & 0.455 & 0.5046 & 0.101 & - & - & - & & & & & & \\
\hline - Sound intensity $\times$ gen. $\times$ coh. & rMeasures & Sph.ass & & & & 0.716 & 0.5922 & 0.079 & - & - & - & & & & & & \\
\hline - Gen. $\times$ coh. & rMeasures & Sph.ass & & & & 1.812 & 0.1783 & 0.353 & - & - & - & & & & & & \\
\hline Pre-pulse inhibition (PPI) & & & & & & & & & & & & & & & & & \\
\hline & & & & & & Genotyp & & & Cohort & & & $\begin{array}{l}\text { Genoty } \\
\text { cohort }\end{array}$ & type $\times$ & & $\begin{array}{l}\text { Pairwis } \\
\text { compar }\end{array}$ & $\begin{array}{l}\text { risons } \\
\text { riso }\end{array}$ & \\
\hline & Test & $\begin{array}{l}\text { Data } \\
\text { structure }\end{array}$ & WT & Het & KO & $F$ & $\begin{array}{l}p \\
\text { value }\end{array}$ & Power & $F$ & $\begin{array}{l}p \\
\text { value }\end{array}$ & Power & $F$ & $\begin{array}{l}p \\
\text { value }\end{array}$ & Power & $\begin{array}{l}\text { WT vs } \\
\text { Het }\end{array}$ & $\begin{array}{l}\text { WT vs } \\
\text { KO }\end{array}$ & $\begin{array}{l}\text { Het } \\
\text { vs KO }\end{array}$ \\
\hline PPI \% (74 dB) & 2WANOVA & Normal & $22.47 \pm 5.7$ & $15.1 \pm 4.72$ & $10.56 \pm 6.03$ & 0.625 & 0.5409 & 0.212 & 11.061 & 0.002 & 0.519 & 1.821 & 0.177 & 0.190 & - & - & - \\
\hline PPI \% (78 dB) & 2WANOVA & Normal & $31.81 \pm 4.48$ & $14.84 \pm 6.7$ & $21.18 \pm 5.03$ & 3.513 & 0.0407 & 0.543 & 21.819 & 0.000 & 0.793 & 1.008 & 0.375 & 0.352 & 0.058 & 0.312 & 0.656 \\
\hline PPI \% (82 dB) & 2WANOVA & Normal & $30.39 \pm 5.51$ & $21.53 \pm 6.08$ & $\begin{array}{r}13.34 \pm 5.99 \\
\quad(\text { Continued })\end{array}$ & 0.151 & 0.8604 & 0.426 & 14.892 & 0.000 & 0.480 & 0.042 & 0.959 & 0.348 & - & - & - \\
\hline
\end{tabular}


Table 7. Continued

\begin{tabular}{|c|c|c|c|c|c|c|c|c|c|c|c|c|c|c|c|c|c|}
\hline & & & & & & \multicolumn{3}{|c|}{ Genotype } & \multicolumn{3}{|l|}{ Cohort } & \multicolumn{3}{|c|}{$\begin{array}{l}\text { Genotype } \times \\
\text { cohort }\end{array}$} & \multicolumn{3}{|c|}{$\begin{array}{l}\text { Pairwise } \\
\text { comparisons }\end{array}$} \\
\hline & Test & $\begin{array}{l}\text { Data } \\
\text { structure }\end{array}$ & WT & Het & KO & $F$ & $\begin{array}{l}p \\
\text { value }\end{array}$ & Power & $F$ & $\begin{array}{l}p \\
\text { value }\end{array}$ & Power & $F$ & $\begin{array}{l}p \\
\text { value }\end{array}$ & Power & $\begin{array}{l}\text { WT vs } \\
\text { Het }\end{array}$ & $\begin{array}{l}\text { WT vs } \\
\text { KO }\end{array}$ & $\begin{array}{l}\text { Het vs } \\
\text { KO }\end{array}$ \\
\hline PPI \% (86 dB) & 2WANOVA & Nonnormal & $31.73 \pm 7.95$ & $21.04 \pm 7.37$ & $27.04 \pm 4.63$ & 1.411 & 0.2575 & 0.176 & 37.139 & 0.000 & 0.909 & 0.463 & 0.633 & 0.217 & - & - & - \\
\hline PPI \% (92 dB) & 2WANOVA & Nonnormal & $43.62 \pm 7.01$ & $34.35 \pm 5.82$ & $29.93 \pm 6.97$ & 0.989 & 0.3821 & 0.212 & 54.683 & 0.000 & 0.892 & 2.258 & 0.120 & 0.222 & - & - & - \\
\hline PPI \% (average) & 2WANOVA & Normal & $32 \pm 5.53$ & $21.37 \pm 5.48$ & $20.41 \pm 5.03$ & 0.155 & 0.8569 & 0.305 & 37.489 & 0.000 & 0.867 & 0.310 & 0.735 & 0.278 & - & - & - \\
\hline PPI & Test & $\begin{array}{l}\text { Data } \\
\text { structure }\end{array}$ & & & & $F$ & $\begin{array}{l}p \\
\text { value }\end{array}$ & Power & $\begin{array}{l}\text { WT vs } \\
\text { Het }\end{array}$ & $\begin{array}{l}\text { WT vs } \\
\text { KO }\end{array}$ & $\begin{array}{l}\text { Het vs } \\
\text { KO }\end{array}$ & & & & & & \\
\hline - Sound intensity & rMeasures & Sph.viol & & & & 15.396 & 0.0000 & 1.000 & - & - & - & & & & & & \\
\hline - Sound intensity $\times$ gen. & rMeasures & Sph.viol & & & & 1.409 & 0.1943 & 0.603 & - & - & - & & & & & & \\
\hline - Genotype & rMeasures & Sph.viol & & & & 1.502 & 0.2325 & 0.305 & - & - & - & & & & & & \\
\hline - Cohort & rMeasures & Sph.viol & & & & 9.806 & 0.0029 & 0.867 & - & - & - & & & & & & \\
\hline - Sound intensity $\times$ gen. $\times$ coh. & rMeasures & Sph.viol & & & & 0.978 & 0.4540 & 0.427 & - & - & - & & & & & & \\
\hline - Gen. $\times$ coh. & rMeasures & Sph.viol & & & & 1.349 & 0.2686 & 0.278 & - & - & - & & & & & & \\
\hline \multicolumn{18}{|l|}{ Burried food test } \\
\hline & & & & & & \multicolumn{3}{|c|}{ Genotype } & \multicolumn{3}{|l|}{ Cohort } & \multicolumn{3}{|c|}{$\begin{array}{l}\text { Genotype } \times \\
\text { cohort }\end{array}$} & \multicolumn{3}{|c|}{$\begin{array}{l}\text { Pairwise } \\
\text { comparisons }\end{array}$} \\
\hline & Test & $\begin{array}{l}\text { Data } \\
\text { structure }\end{array}$ & WT & Het & KO & $F$ & $\begin{array}{l}p \\
\text { value }\end{array}$ & Power & $F$ & $\begin{array}{l}p \\
\text { value }\end{array}$ & Power & $F$ & $\begin{array}{l}p \\
\text { value }\end{array}$ & Power & $\begin{array}{l}\text { WT vs } \\
\text { Het }\end{array}$ & $\begin{array}{l}\text { WT vs } \\
\text { KO }\end{array}$ & $\begin{array}{l}\text { Het } \\
\text { vs KO }\end{array}$ \\
\hline $\begin{array}{l}\text { Latency to retrieve and eat } \\
\text { food (s) }\end{array}$ & 2WANOVA & Nonnormal & $51.11 \pm 9.9$ & $94.81 \pm 23.69$ & $500.28 \pm 94.92$ & 17.848 & 0.0000 & 1.000 & 0.001 & 0.976 & 0.050 & 0.000 & 1.000 & 0.050 & 0.858 & 0.000 & 0.000 \\
\hline \multicolumn{18}{|c|}{ Olfactory habituation/dishabituation, sniffing only } \\
\hline Water & Test & $\begin{array}{l}\text { Data } \\
\text { structure }\end{array}$ & & & & $F$ & $\begin{array}{l}p \\
\text { value }\end{array}$ & Power & $\begin{array}{l}\text { WT vs } \\
\text { Het }\end{array}$ & $\begin{array}{l}\text { WT vs } \\
\text { KO }\end{array}$ & $\begin{array}{l}\text { Het } \\
\text { vs KO }\end{array}$ & & & & & & \\
\hline - Trial & rMeasures & Sph.viol & & & & 8.290 & 0.0019 & 0.958 & - & - & - & & & & & & \\
\hline - Trial $\times$ gen & rMeasures & Sph.viol & & & & 1.108 & 0.3505 & 0.337 & - & - & - & & & & & & \\
\hline - Genotype & rMeasures & Sph.viol & & & & 2.973 & 0.0602 & 0.553 & 0.660 & 0.027 & 0.066 & & & & & & \\
\hline - Cohort & rMeasures & Sph.viol & & & & 0.073 & 0.7886 & 0.058 & - & - & - & & & & & & \\
\hline - Trial $\times$ gen. $\times$ coh & rMeasures & Sph.viol & & & & 1.739 & 0.1683 & 0.515 & - & - & - & & & & & & \\
\hline - Gen. $\times$ coh. & rMeasures & Sph.viol & & & & 1.015 & 0.3696 & 0.217 & - & - & - & & & & & & \\
\hline Banana & Test & $\begin{array}{l}\text { Data } \\
\text { structure }\end{array}$ & & & & $F$ & $\begin{array}{l}p \\
\text { value }\end{array}$ & Power & $\begin{array}{l}\text { WT vs } \\
\text { Het }\end{array}$ & $\begin{array}{l}\text { WT vs } \\
\text { KO }\end{array}$ & $\begin{array}{l}\text { Het } \\
\text { vs KO }\end{array}$ & & & & & & \\
\hline - Trial & rMeasures & Sph.ass & & & & 10.117 & 0.0001 & 0.983 & - & - & - & & & & & & \\
\hline - Trial $\times$ gen & rMeasures & Sph.ass & & & & 3.908 & 0.0054 & 0.888 & - & - & - & & & & & & \\
\hline - Genotype & rMeasures & Sph.ass & & & & 5.681 & 0.0060 & 0.842 & 0.433 & 0.002 & 0.017 & & & & & & \\
\hline - Cohort & rMeasures & Sph.ass & & & & 11.933 & 0.0011 & 0.923 & - & - & - & & & & & & \\
\hline - Trial $\times$ gen. $\times$ coh & rMeasures & Sph.ass & & & & 0.486 & 0.7461 & 0.161 & - & - & - & & & & & & \\
\hline - Gen. $\times$ coh. & rMeasures & Sph.ass & & & & 0.134 & 0.8752 & 0.069 & - & - & - & & & & & & \\
\hline Lemon & Test & $\begin{array}{l}\text { Data } \\
\text { structure }\end{array}$ & & & & $F$ & $\begin{array}{l}p \\
\text { value }\end{array}$ & Power & $\begin{array}{l}\text { WT vs } \\
\text { Het }\end{array}$ & $\begin{array}{l}\text { WT vs } \\
\text { KO }\end{array}$ & $\begin{array}{l}\text { Het } \\
\text { vs KO }\end{array}$ & & & & & & \\
\hline - Trial & rMeasures & Sph.viol & & & & 6.699 & 0.0041 & 0.908 & - & - & - & & & & & & \\
\hline - Trial $\times$ gen & rMeasures & Sph.viol & & & & 0.047 & 0.9890 & 0.059 & - & - & - & & & & & & \\
\hline - Genotype & rMeasures & Sph.viol & & & & 2.715 & 0.0760 & 0.513 & 0.404 & 0.166 & 0.025 & & & & & & \\
\hline - Cohort & rMeasures & Sph.viol & & & & 15.327 & 0.0003 & 0.970 & - & - & - & & & & & & \\
\hline - Trial $\times$ gen. $\times$ coh & rMeasures & Sph.viol & & & & 0.667 & 0.5828 & 0.211 & - & - & - & & & & & & \\
\hline - Gen. × coh. & rMeasures & Sph.viol & & & & 0.159 & 0.8534 & 0.073 & - & - & - & & & & & & \\
\hline Male scent & Test & $\begin{array}{l}\text { Data } \\
\text { structure }\end{array}$ & & & & $F$ & $\begin{array}{l}p \\
\text { value }\end{array}$ & Power & $\begin{array}{l}\text { WT vs } \\
\text { Het }\end{array}$ & $\begin{array}{l}\text { WT vs } \\
\text { KO }\end{array}$ & $\begin{array}{l}\text { Het vs } \\
\text { KO }\end{array}$ & & & & & & \\
\hline - Trial & rMeasures & Sph.viol & & & & 27.903 & 0.0000 & 1.000 & - & - & - & & & & & & \\
\hline - Trial $\times$ gen & rMeasures & Sph.viol & & & & 1.089 & 0.3581 & 0.332 & - & - & - & & & & & & \\
\hline - Genotype & rMeasures & Sph.viol & & & & 0.739 & 0.4828 & 0.168 & - & - & - & & & & & & \\
\hline - Cohort & rMeasures & Sph.viol & & & & 2.500 & 0.1201 & 0.341 & - & - & - & & & & & & \\
\hline - Trial $\times$ gen. $\times$ coh & rMeasures & Sph.viol & & & & 1.603 & 0.1976 & 0.479 & - & - & - & & & & & & \\
\hline - Gen. $\times$ coh. & rMeasures & Sph.viol & & & & 0.541 & 0.5857 & 0.134 & - & - & - & & & & & & \\
\hline Female scent & Test & $\begin{array}{l}\text { Data } \\
\text { structure }\end{array}$ & & & & $F$ & $\begin{array}{l}p \\
\text { value }\end{array}$ & Power & $\begin{array}{l}\text { WT vs } \\
\text { Het }\end{array}$ & $\begin{array}{l}\text { WT vs } \\
\text { KO }\end{array}$ & $\begin{array}{l}\text { Het vs } \\
\text { KO }\end{array}$ & & & & & & \\
\hline - Trial & rMeasures & Sph.viol & & & & 20.922 & 0.0000 & 1.000 & - & - & - & & & & & & \\
\hline - Trial $\times$ gen & rMeasures & Sph.viol & & & & 0.131 & 0.9321 & 0.076 & - & - & - & & & & & & \\
\hline - Genotype & rMeasures & Sph.viol & & & & 0.585 & 0.5609 & 0.142 & - & - & - & & & & & & \\
\hline - Cohort & rMeasures & Sph.viol & & & & 14.771 & 0.0003 & 0.965 & - & - & - & & & & & & \\
\hline - Trial $\times$ gen. $\times$ coh & rMeasures & Sph.viol & & & & 0.348 & 0.7765 & 0.126 & - & - & - & & & & & & \\
\hline - Gen. $\times$ coh. & rMeasures & Sph.viol & & & & 1.651 & 0.2022 & 0.332 & - & - & - & & & & & & \\
\hline & & & & & & Genotyp & & & Cohort & & & $\begin{array}{l}\text { Genoty } \\
\text { cohort }\end{array}$ & type $\times$ & & $\begin{array}{l}\text { Pairwise } \\
\text { compar }\end{array}$ & $\begin{array}{l}\text { risons } \\
\text { riso }\end{array}$ & \\
\hline Individual trials & Test & $\begin{array}{l}\text { Data } \\
\text { structure }\end{array}$ & WT & Het & KO & $F$ & $\begin{array}{l}p \\
\text { value }\end{array}$ & Power & $F$ & $\begin{array}{l}p \\
\text { value }\end{array}$ & Power & $F$ & $\begin{array}{l}p \\
\text { value }\end{array}$ & Power & $\begin{array}{l}\text { WT vs } \\
\text { Het }\end{array}$ & $\begin{array}{l}\text { WT vs } \\
\text { KO }\end{array}$ & $\begin{array}{l}\text { Het } \\
\text { vs KO }\end{array}$ \\
\hline Water 1 & 2WANOVA & Nonnormal & $1.82 \pm 0.37$ & $1.81 \pm 0.42$ & $0.9 \pm 0.16$ & 2.205 & 0.1208 & 0.429 & 0.199 & 0.658 & 0.072 & 1.697 & 0.194 & 0.340 & - & - & - \\
\hline Water 2 & 2WANOVA & Nonnormal & $1.02 \pm 0.15$ & $0.98 \pm 0.14$ & $0.7 \pm 0.15$ & 1.438 & 0.2470 & 0.294 & 0.078 & 0.781 & 0.059 & 0.711 & 0.496 & 0.163 & - & - & - \\
\hline Water 3 & 2WANOVA & Nonnormal & $0.97 \pm 0.15$ & $0.82 \pm 0.14$ & $0.7 \pm 0.11$ & 1.724 & 0.1888 & 0.345 & 2.900 & 0.095 & 0.386 & 0.366 & 0.696 & 0.106 & - & - & - \\
\hline Banana 1 & 2WANOVA & Nonnormal & $1.34 \pm 0.12$ & $1.04 \pm 0.23$ & $0.44 \pm 0.15$ & 8.742 & 0.0006 & 0.961 & 6.201 & 0.016 & 0.685 & 0.466 & 0.630 & 0.122 & 0.322 & 0.001 & 0.052 \\
\hline Banana 2 & 2WANOVA & Nonnormal & $0.65 \pm 0.12$ & $0.65 \pm 0.1$ & $0.44 \pm 0.13$ & 1.641 & 0.2040 & 0.330 & 6.658 & 0.013 & 0.716 & 0.240 & 0.787 & 0.086 & - & - & - \\
\hline Banana 3 & 2WANOVA & Nonnormal & $0.52 \pm 0.09$ & $0.72 \pm 0.19$ & $\begin{aligned} 0.44 & \pm 0.13 \\
& \text { (Continued) }\end{aligned}$ & 1.167 & 0.3196 & 0.245 & 7.761 & 0.008 & 0.780 & 0.050 & 0.951 & 0.057 & - & - & - \\
\hline
\end{tabular}


Table 7. Continued

\begin{tabular}{|c|c|c|c|c|c|c|c|c|c|c|c|c|c|c|c|c|c|}
\hline & \multirow[b]{2}{*}{ Test } & \multirow[b]{2}{*}{$\begin{array}{l}\text { Data } \\
\text { structure }\end{array}$} & \multirow[b]{2}{*}{ WT } & \multirow[b]{2}{*}{ Het } & \multirow[b]{2}{*}{$\mathrm{KO}$} & \multicolumn{3}{|c|}{ Genotype } & \multicolumn{3}{|l|}{ Cohort } & \multicolumn{3}{|c|}{$\begin{array}{l}\text { Genotype } \times \\
\text { cohort }\end{array}$} & $\begin{array}{l}\text { Pairwise } \\
\text { compari }\end{array}$ & $\begin{array}{l}\text { e } \\
\text { isons }\end{array}$ & \\
\hline & & & & & & $F$ & $\begin{array}{l}p \\
\text { value }\end{array}$ & Power & $F$ & $\begin{array}{l}p \\
\text { value }\end{array}$ & Power & $F$ & $\begin{array}{l}p \\
\text { value }\end{array}$ & Power & $\begin{array}{l}\text { WT vs } \\
\text { Het }\end{array}$ & $\begin{array}{l}\text { WT vs } \\
\text { KO }\end{array}$ & $\begin{array}{l}\text { Het vs } \\
\mathrm{KO}\end{array}$ \\
\hline Lemon 1 & 2wANOVA & Nonnormal & $0.58 \pm 0.18$ & $0.77 \pm 0.15$ & $0.52 \pm 0.17$ & 0.720 & 0.4916 & 0.165 & 8.572 & 0.005 & 0.819 & 0.041 & 0.960 & 0.056 & - & - & - \\
\hline Lemon 2 & 2WANOVA & Nonnormal & $0.39 \pm 0.11$ & $0.52 \pm 0.1$ & $0.24 \pm 0.07$ & 2.679 & 0.0785 & 0.507 & 9.784 & 0.003 & 0.866 & 0.037 & 0.964 & 0.055 & 0.709 & 0.411 & 0.099 \\
\hline Lemon 3 & 2wANOVA & Nonnormal & $0.35 \pm 0.06$ & $0.48 \pm 0.09$ & $0.25 \pm 0.08$ & 2.547 & 0.0885 & 0.486 & 7.405 & 0.009 & 0.761 & 2.493 & 0.093 & 0.478 & 0.594 & 0.540 & 0.106 \\
\hline Male 1 & 2wANOVA & Nonnormal & $6.1 \pm 1.12$ & $4.66 \pm 0.88$ & $4.94 \pm 1.07$ & 0.659 & 0.5219 & 0.154 & 1.095 & 0.300 & 0.177 & 1.091 & 0.344 & 0.231 & - & - & - \\
\hline Male 2 & 2wANOVA & Nonnormal & $2.25 \pm 0.34$ & $2.77 \pm 0.46$ & $1.26 \pm 0.31$ & 4.095 & 0.0225 & 0.700 & 0.564 & 0.456 & 0.114 & 3.078 & 0.055 & 0.568 & 0.752 & 0.116 & 0.020 \\
\hline Male 3 & 2wANOVA & Nonnormal & $1.54 \pm 0.38$ & $1.25 \pm 0.24$ & $1.72 \pm 0.63$ & 0.322 & 0.7264 & 0.098 & 2.465 & 0.123 & 0.337 & 0.312 & 0.733 & 0.097 & - & - & - \\
\hline Female 1 & 2wANOVA & Nonnormal & $5.68 \pm 0.98$ & $6.43 \pm 1.34$ & $6.32 \pm 1.93$ & 0.129 & 0.8790 & 0.069 & 11.509 & 0.001 & 0.914 & 0.879 & 0.422 & 0.193 & - & - & - \\
\hline Female 2 & 2WANOVA & Nonnormal & $2.18 \pm 0.3$ & $2.24 \pm 0.43$ & $2.62 \pm 0.81$ & 0.189 & 0.8283 & 0.078 & 1.936 & 0.170 & 0.276 & 0.289 & 0.751 & 0.093 & - & - & - \\
\hline Female 3 & 2wANOVA & Nonnormal & $1.42 \pm 0.26$ & $1.82 \pm 0.52$ & $2.76 \pm 0.88$ & 1.600 & 0.2120 & 0.323 & 7.672 & 0.008 & 0.775 & 1.792 & 0.177 & 0.357 & - & - & - \\
\hline Olfactory habituation & /dishabituat & tion, all int & actions & & & & & & & & & & & & & & \\
\hline Water & Test & $\begin{array}{l}\text { Data } \\
\text { structure }\end{array}$ & & & & $F$ & $\begin{array}{l}p \\
\text { value }\end{array}$ & Power & $\begin{array}{l}\text { WT vs } \\
\text { Het }\end{array}$ & $\begin{array}{l}\text { WT vs } \\
\text { KO }\end{array}$ & $\begin{array}{l}\text { Het } \\
\text { vs KO }\end{array}$ & & & & & & \\
\hline - Trial & rMeasures & Sph.viol & & & & 1.891 & 0.1664 & 0.385 & - & - & - & & & & & & \\
\hline - Trial $\times$ gen & rMeasures & Sph.viol & & & & 0.718 & 0.5478 & 0.225 & - & - & - & & & & & & \\
\hline - Genotype & rMeasures & Sph.viol & & & & 7.210 & 0.0017 & 0.920 & 0.637 & 0.001 & 0.015 & & & & & & \\
\hline - Cohort & rMeasures & Sph.viol & & & & 2.639 & 0.1104 & 0.357 & - & - & - & & & & & & \\
\hline - Trial $\times$ gen. $\times$ coh & rMeasures & Sph.viol & & & & 0.920 & 0.4371 & 0.283 & - & - & - & & & & & & \\
\hline - Gen. × coh. & rMeasures & Sph.viol & & & & 1.133 & 0.3300 & 0.239 & - & - & - & & & & & & \\
\hline Banana & Test & $\begin{array}{l}\text { Data } \\
\text { structure }\end{array}$ & & & & $F$ & $\begin{array}{l}p \\
\text { value }\end{array}$ & Power & $\begin{array}{l}\text { WT vs } \\
\text { Het }\end{array}$ & $\begin{array}{l}\text { WT vs } \\
\text { KO }\end{array}$ & $\begin{array}{l}\text { Het vs } \\
\text { KO }\end{array}$ & & & & & & \\
\hline - Trial & rMeasures & Sph.viol & & & & 5.229 & 0.0133 & 0.821 & - & - & - & & & & & & \\
\hline - Trial $\times$ gen & rMeasures & Sph.viol & & & & 1.282 & 0.2866 & 0.388 & - & - & - & & & & & & \\
\hline - Genotype & rMeasures & Sph.viol & & & & 5.737 & 0.0057 & 0.846 & 0.744 & 0.060 & 0.008 & & & & & & \\
\hline - Cohort & rMeasures & Sph.viol & & & & 7.922 & 0.0070 & 0.788 & - & - & - & & & & & & \\
\hline - Trial $\times$ gen. $\times$ coh & rMeasures & Sph.viol & & & & 0.936 & 0.4284 & 0.287 & - & - & - & & & & & & \\
\hline - Gen. $\times$ coh & rMeasures & Sph.viol & & & & 0.990 & 0.3787 & 0.213 & - & - & - & & & & & & \\
\hline Lemon & Test & $\begin{array}{l}\text { Data } \\
\text { structure }\end{array}$ & & & & $F$ & $\begin{array}{l}p \\
\text { value }\end{array}$ & Power & $\begin{array}{l}\text { WT vs } \\
\text { Het }\end{array}$ & $\begin{array}{l}\text { WT vs } \\
\text { KO }\end{array}$ & $\begin{array}{l}\text { Het } \\
\text { vs KO }\end{array}$ & & & & & & \\
\hline - Trial & rMeasures & Sph.viol & & & & 1.303 & 0.2728 & 0.276 & - & - & - & & & & & & \\
\hline - Trial $\times$ gen & rMeasures & Sph.viol & & & & 0.703 & 0.5597 & 0.221 & - & - & - & & & & & & \\
\hline - Genotype & rMeasures & Sph.viol & & & & 4.893 & 0.0115 & 0.781 & 0.295 & 0.048 & 0.003 & & & & & & \\
\hline - Cohort & rMeasures & Sph.viol & & & & 5.152 & 0.0276 & 0.605 & - & - & - & & & & & & \\
\hline - Trial $\times$ gen. $\times$ coh & rMeasures & Sph.viol & & & & 0.527 & 0.6738 & 0.172 & - & - & - & & & & & & \\
\hline - Gen. $\times$ coh. & rMeasures & Sph.viol & & & & 2.405 & 0.1006 & 0.463 & - & - & - & & & & & & \\
\hline Male scent & Test & $\begin{array}{l}\text { Data } \\
\text { structure }\end{array}$ & & & & $F$ & $\begin{array}{l}p \\
\text { value }\end{array}$ & Power & $\begin{array}{l}\text { WT vs } \\
\text { Het }\end{array}$ & $\begin{array}{l}\text { WT vs } \\
\text { KO }\end{array}$ & $\begin{array}{l}\text { Het } \\
\text { vs KO }\end{array}$ & & & & & & \\
\hline - Trial & rMeasures & Sph.viol & & & & 28.652 & 0.0000 & 1.000 & - & - & - & & & & & & \\
\hline - Trial $\times$ gen & rMeasures & Sph.viol & & & & 0.790 & 0.4993 & 0.246 & - & - & - & & & & & & \\
\hline - Genotype & rMeasures & Sph.viol & & & & 5.722 & 0.0057 & 0.845 & 0.839 & 0.005 & 0.022 & & & & & & \\
\hline - Cohort & rMeasures & Sph.viol & & & & 2.953 & 0.0918 & 0.392 & - & - & - & & & & & & \\
\hline - Trial $\times$ gen. $\times$ coh & rMeasures & Sph.viol & & & & 0.367 & 0.7697 & 0.131 & - & - & - & & & & & & \\
\hline - Gen. × coh. & rMeasures & Sph.viol & & & & 0.009 & 0.9906 & 0.051 & - & - & - & & & & & & \\
\hline Female scent & Test & $\begin{array}{l}\text { Data } \\
\text { structure }\end{array}$ & & & & $F$ & $\begin{array}{l}p \\
\text { value }\end{array}$ & Power & $\begin{array}{l}\text { WT vs } \\
\text { Het }\end{array}$ & $\begin{array}{l}\text { WT vs } \\
\text { KO }\end{array}$ & $\begin{array}{l}\text { Het } \\
\text { vs KO }\end{array}$ & & & & & & \\
\hline - Trial & rMeasures & Sph.ass & & & & 25.044 & 0.0000 & 1.000 & - & - & - & & & & & & \\
\hline - Trial $\times$ gen & rMeasures & Sph.ass & & & & 0.884 & 0.4762 & 0.264 & - & - & - & & & & & & \\
\hline - Genotype & rMeasures & Sph.ass & & & & 1.119 & 0.3346 & 0.236 & - & - & - & & & & & & \\
\hline - Cohort & rMeasures & Sph.ass & & & & 6.400 & 0.0145 & 0.699 & - & - & - & & & & & & \\
\hline - Trial $\times$ gen. $\times$ coh & rMeasures & Sph.ass & & & & 1.911 & 0.1143 & 0.541 & - & - & - & & & & & & \\
\hline - Gen. $\times$ coh. & rMeasures & Sph.ass & & & & 0.431 & 0.6521 & 0.116 & - & - & - & & & & & & \\
\hline & & & & & & Genotyp & & & Cohort & & & $\begin{array}{l}\text { Genoty } \\
\text { cohort }\end{array}$ & $e \times$ & & $\begin{array}{l}\text { Pairwise } \\
\text { compar }\end{array}$ & $\begin{array}{l}\text { e } \\
\text { risons }\end{array}$ & \\
\hline Individual trials & Test & $\begin{array}{l}\text { Data } \\
\text { structure }\end{array}$ & WT & Het & $\mathrm{KO}$ & $F$ & $\begin{array}{l}p \\
\text { value }\end{array}$ & Power & $F$ & $\begin{array}{l}p \\
\text { value }\end{array}$ & Power & $F$ & $\begin{array}{l}p \\
\text { value }\end{array}$ & Power & $\begin{array}{l}\text { WT vs } \\
\text { Het }\end{array}$ & $\begin{array}{l}\text { WT vs } \\
\text { KO }\end{array}$ & $\begin{array}{l}\text { Het } \\
\text { vs KO }\end{array}$ \\
\hline Water 1 & 2WANOVA & Nonnormal & $3.52 \pm 0.9$ & $3.88 \pm 1$ & $1.35 \pm 0.27$ & 2.357 & 0.1053 & 0.455 & 3.302 & 0.075 & 0.429 & 1.116 & 0.336 & 0.235 & - & - & - \\
\hline Water 2 & 2wANOVA & Nonnormal & $3.45 \pm 0.71$ & $1.78 \pm 0.48$ & $0.95 \pm 0.23$ & 4.964 & 0.0109 & 0.786 & 0.588 & 0.447 & 0.117 & 1.295 & 0.283 & 0.267 & 0.161 & 0.006 & 0.364 \\
\hline Water 3 & 2WANOVA & Nonnormal & $2.53 \pm 0.54$ & $2.3 \pm 0.6$ & $0.95 \pm 0.2$ & 2.997 & 0.0592 & 0.556 & 0.033 & 0.856 & 0.054 & 0.373 & 0.690 & 0.107 & 0.949 & 0.078 & 0.147 \\
\hline Banana 1 & 2wANOVA & Nonnormal & $2.8 \pm 0.42$ & $3.38 \pm 1.16$ & $0.72 \pm 0.43$ & 3.854 & 0.0279 & 0.672 & 6.603 & 0.013 & 0.712 & 1.080 & 0.348 & 0.229 & - & - & - \\
\hline Banana 2 & 2WANOVA & Nonnormal & $1.46 \pm 0.49$ & $1.06 \pm 0.35$ & $0.57 \pm 0.18$ & 1.902 & 0.1601 & 0.376 & 4.991 & 0.030 & 0.591 & 1.056 & 0.355 & 0.224 & - & - & - \\
\hline Banana 3 & 2WANOVA & Nonnormal & $1.38 \pm 0.55$ & $2 \pm 0.63$ & $0.4 \pm 0.1$ & 3.927 & 0.0262 & 0.680 & 1.920 & 0.172 & 0.274 & 1.097 & 0.342 & 0.232 & 0.334 & 0.380 & 0.020 \\
\hline Lemon 1 & 2WANOVA & Nonnormal & $1.47 \pm 0.37$ & $2.21 \pm 0.74$ & $0.45 \pm 0.19$ & 3.707 & 0.0317 & 0.653 & 2.710 & 0.106 & 0.365 & 0.878 & 0.422 & 0.193 & 0.412 & 0.339 & 0.024 \\
\hline Lemon 2 & 2WANOVA & Nonnormal & $1.21 \pm 0.35$ & $0.98 \pm 0.36$ & $0.28 \pm 0.09$ & 2.536 & 0.0895 & 0.484 & 3.609 & 0.063 & 0.461 & 1.012 & 0.371 & 0.216 & 0.781 & 0.091 & 0.315 \\
\hline Lemon 3 & 2WANOVA & Nonnormal & $0.8 \pm 0.18$ & $0.61 \pm 0.15$ & $0.2 \pm 0.08$ & 1.362 & 0.2656 & 0.280 & 1.615 & 0.210 & 0.238 & 1.334 & 0.273 & 0.275 & - & - & - \\
\hline Male 1 & 2WANOVA & Nonnormal & $55.55 \pm 6.73$ & $48.98 \pm 7.22$ & $27.15 \pm 6.11$ & 3.341 & 0.0436 & 0.605 & 2.419 & 0.126 & 0.332 & 0.054 & 0.948 & 0.058 & 0.655 & 0.030 & 0.199 \\
\hline Male 2 & 2WANOVA & Nonnormal & $27.09 \pm 6.01$ & $29.19 \pm 5.33$ & $7.48 \pm 4.34$ & 4.753 & 0.0130 & 0.768 & 1.196 & 0.280 & 0.189 & 0.012 & 0.988 & 0.052 & 0.986 & 0.020 & 0.030 \\
\hline Male 3 & 2WANOVA & Nonnormal & $17.57 \pm 5.5$ & $14.39 \pm 4.43$ & $5.26 \pm 1.63$ & 2.323 & 0.1087 & 0.449 & 1.329 & 0.255 & 0.204 & 0.978 & 0.383 & 0.210 & - & - & - \\
\hline Female 1 & 2WANOVA & Nonnormal & $53.65 \pm 5.97$ & $50.59 \pm 5.48$ & $31.16 \pm 7.21$ & 1.781 & 0.1792 & 0.355 & 6.118 & 0.017 & 0.679 & 1.565 & 0.219 & 0.316 & - & - & - \\
\hline Female 2 & 2WANOVA & Nonnormal & $23.26 \pm 6.37$ & $18.85 \pm 6.77$ & $14.61 \pm 4.3$ & 0.427 & 0.6551 & 0.115 & 2.116 & 0.152 & 0.297 & 0.350 & 0.707 & 0.103 & - & - & - \\
\hline Female 3 & 2WANOVA & Nonnormal & $14.57 \pm 3.85$ & $11.5 \pm 3.69$ & $14.21 \pm 4.61$ & 0.269 & 0.7650 & 0.090 & 0.575 & 0.452 & 0.115 & 1.703 & 0.193 & 0.341 & - & - & - \\
\hline
\end{tabular}

WT, wild-type mice; Het, heterozygous mice; KO, homozygous knock-out mice. Group values are reported as mean \pm SEM. Bold font indicates significant results $(p<0.05)$. Individual results and statistical analyses for cohorts 1 and 2 are available in Extended Data Table 7-1. 2wANOVA: 2-way ANOVA, rMeasures: repeated measures, Sph.ass: sphericity assumed, Sph.viol: sphericity violated, gen: genotype, coh: cohort. 

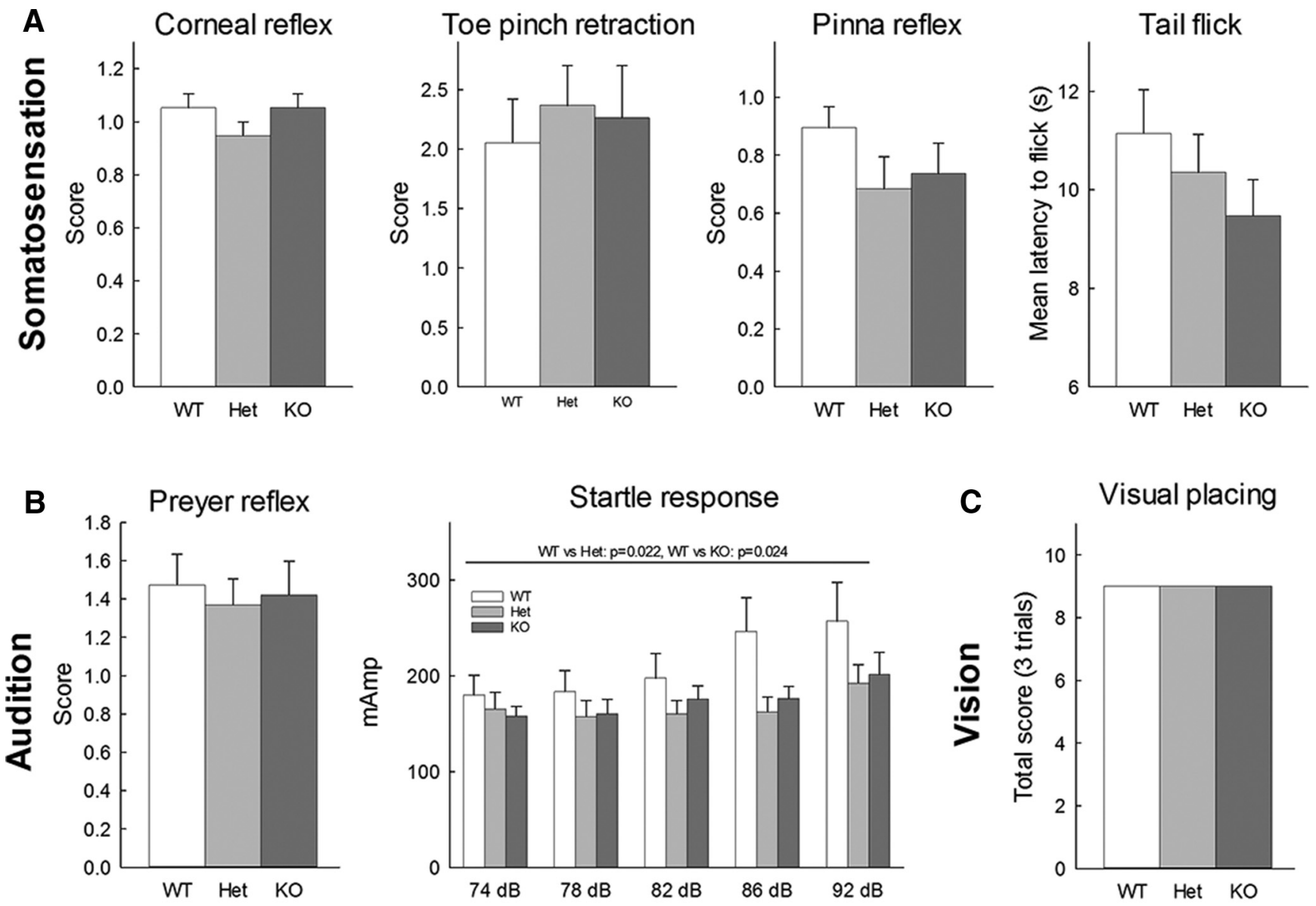

D

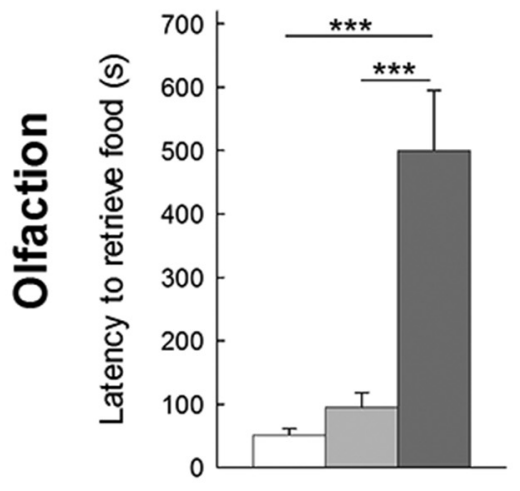

\section{Olfactory habituation/dishabituation}

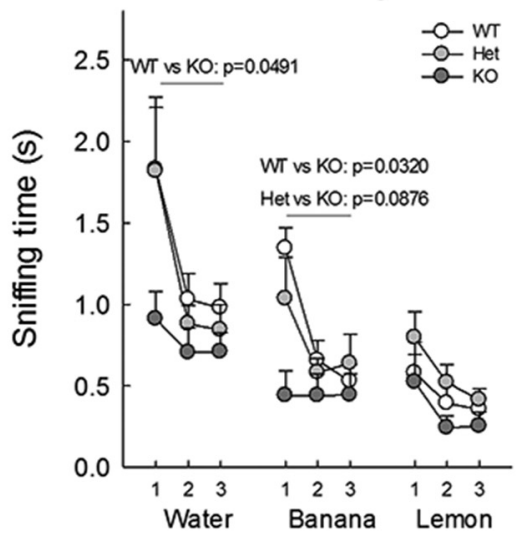

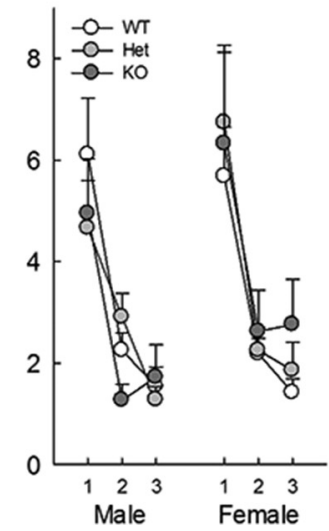

Figure 4. Altered sensory profile in Shank $3^{\Delta 4-22}$-deficient mice. $\boldsymbol{A}$, Somatosensation evaluated with corneal reflex, toe pinch retraction, pinna reflex, and tail flick. Normal tactile and pain responses were observed in Shank $3^{\Delta 4-22}$-deficient mice. $\boldsymbol{B}$, Auditory functions measured with the Preyer reflex and startle response to increasing sound intensities. No genotype difference was observed for Preyer reflex, however startle response was decreased in both heterozygous and homozygous Shank $3^{\Delta 4-22}$ mice compared to their wild-type littermate with genotype differences being more marked for the higher startle intensities. Pre-pulse inhibition results are displayed in Extended Data Figure 4-1A. C, Gross visual function assessed by the visual placing test. Normal visual placing was observed for all genotypes. $\boldsymbol{D}$, Olfactory abilities evaluated by the time to find hidden food in buried food test and the cumulative time sniffing the applicator without direct interactions during olfactory habituation and dishabituation to nonsocial and social odors. Strong impairments were observed in the buried food test for Shank $3^{\Delta 4-22}$ homozygous mice as shown by a significant increase in the latency to retrieve the buried food, compared to their heterozygous and wild-type littermates. Individual performances are available in Extended Data Figure 4-1B. Similarly, a significant lack of interest for nonsocial scents (water, banana, and lemon) was observed in Shank $3^{\Delta-22}$ homozygous mice but not in heterozygotes and wild-type during olfactory habituation/dishabituation, while they still 
Table 8. Detailed results and statistical analyses related to social behavior

\begin{tabular}{|c|c|c|c|c|c|c|c|c|c|c|c|c|c|c|c|c|c|}
\hline \multicolumn{18}{|c|}{ Three chambered social interaction test - social preference } \\
\hline \multicolumn{18}{|c|}{ Zone comparison, three zones, time in chambers } \\
\hline All mice & Test & \multicolumn{4}{|l|}{$\begin{array}{l}\text { Data } \\
\text { structure }\end{array}$} & $F$ & $\begin{array}{l}p \\
\text { value }\end{array}$ & Power & $\begin{array}{l}\text { C vs } \\
M\end{array}$ & $\begin{array}{l}\text { C vs } \\
\mathrm{O}\end{array}$ & \multicolumn{7}{|l|}{$\begin{array}{l}M \text { vs } \\
O\end{array}$} \\
\hline - Chamber & rMeasures & \multicolumn{4}{|l|}{ Sph.viol } & 149.525 & 0.0000 & 1.000 & 0.000 & 0.000 & \multicolumn{7}{|l|}{0.000} \\
\hline - Cohort & rMeasures & \multicolumn{4}{|l|}{ Sph.viol } & 1.456 & 0.2328 & 0.452 & - & - & \multicolumn{7}{|l|}{-} \\
\hline - Chamber $\times$ cohort & rMeasures & \multicolumn{4}{|l|}{ Sph.viol } & 2.267 & 0.1149 & 0.220 & - & - & \multicolumn{7}{|l|}{-} \\
\hline WT & Test & \multicolumn{4}{|l|}{$\begin{array}{l}\text { Data } \\
\text { structure }\end{array}$} & $F$ & $\begin{array}{l}p \\
\text { value }\end{array}$ & Power & $\begin{array}{l}\text { WT vs } \\
\text { Het }\end{array}$ & $\begin{array}{l}\text { WT vs } \\
\text { KO }\end{array}$ & $\begin{array}{l}\text { Het } \\
\text { vs KO }\end{array}$ & & & & & & \\
\hline - Chamber & rMeasures & Sph.ass & & & & 78.786 & 0.0000 & 1.000 & 0.000 & 0.001 & 0.000 & & & & & & \\
\hline - Cohort & rMeasures & Sph.ass & & & & 5.360 & 0.0342 & 0.585 & - & - & - & & & & & & \\
\hline - Chamber $\times$ cohort & rMeasures & Sph.ass & & & & 1.546 & 0.2285 & 0.297 & - & - & - & & & & & & \\
\hline Het & Test & $\begin{array}{l}\text { Data } \\
\text { structure }\end{array}$ & & & & $F$ & $\begin{array}{l}p \\
\text { value }\end{array}$ & Power & $\begin{array}{l}\text { WT vs } \\
\text { Het }\end{array}$ & $\begin{array}{l}\text { WT vs } \\
\text { KO }\end{array}$ & $\begin{array}{l}\text { Het vs } \\
\text { KO }\end{array}$ & & & & & & \\
\hline - Chamber & rMeasures & Sph.viol & & & & 61.909 & 0.0000 & 1.000 & 0.000 & 0.001 & 0.000 & & & & & & \\
\hline - Cohort & rMeasures & Sph.viol & & & & 1.252 & 0.2787 & 0.184 & - & - & - & & & & & & \\
\hline - Chamber $\times$ cohort & rMeasures & Sph.viol & & & & 3.768 & 0.0508 & 0.543 & - & - & - & & & & & & \\
\hline KO & Test & $\begin{array}{l}\text { Data } \\
\text { structure }\end{array}$ & & & & $F$ & $\begin{array}{l}p \\
\text { value }\end{array}$ & Power & $\begin{array}{l}\text { WT vs } \\
\text { Het }\end{array}$ & $\begin{array}{l}\text { WT vs } \\
\text { KO }\end{array}$ & $\begin{array}{l}\text { Het vs } \\
\text { KO }\end{array}$ & & & & & & \\
\hline - Chamber & rMeasures & Sph.ass & & & & 30.043 & 0.0000 & 1.000 & 0.000 & 0.002 & 0.000 & & & & & & \\
\hline - Cohort & rMeasures & Sph.ass & & & & 2.003 & 0.1751 & 0.267 & - & - & - & & & & & & \\
\hline - Chamber $\times$ cohort & rMeasures & Sph.ass & & & & 0.227 & 0.7982 & 0.082 & - & - & - & & & & & & \\
\hline Zone comparison, two zor & nes, Mouse & A vs object $i$ & interaction time & & & & & & & & & & & & & & \\
\hline & Test & $\begin{array}{l}\text { Data } \\
\text { structure }\end{array}$ & & & & All $F$ & $\begin{array}{l}\text { All } p \\
\text { value }\end{array}$ & Power & WT $F$ & $\begin{array}{l}\text { WT } p \\
\text { value }\end{array}$ & Power & Het $F$ & $\begin{array}{l}\text { Het } p \\
\text { value }\end{array}$ & Power & KO $F$ & $\begin{array}{l}\text { KO } p \\
\text { value }\end{array}$ & Power \\
\hline - Chamber & rMeasures & Sph.ass & & & & 40.069 & 0.0000 & 1.000 & 10.622 & 0.005 & 0.864 & 14.120 & 0.002 & 0.943 & 19.123 & 0.000 & 0.984 \\
\hline - Cohort & rMeasures & Sph.ass & & & & 1.078 & 0.3038 & 0.175 & 0.561 & 0.465 & 0.109 & 3.631 & 0.769 & 0.059 & 0.434 & 0.519 & 0.095 \\
\hline - Chamber $\times$ cohort & rMeasures & Sph.ass & & & & 0.921 & 0.3414 & 0.156 & 0.002 & 0.963 & 0.050 & 0.089 & 0.074 & 0.436 & 0.617 & 0.443 & 0.115 \\
\hline & & & & & & Genotype & & & Cohort & & & $\begin{array}{l}\text { Genoty } \\
\text { cohort }\end{array}$ & pe $\times$ & & $\begin{array}{l}\text { Pairwise } \\
\text { compari }\end{array}$ & $\begin{array}{l}\text { e } \\
\text { risons }\end{array}$ & \\
\hline & Test & $\begin{array}{l}\text { Data } \\
\text { structure }\end{array}$ & WT & Het & KO & $F$ & $\begin{array}{l}p \\
\text { value }\end{array}$ & Power & $F$ & $\begin{array}{l}p \\
\text { value }\end{array}$ & Power & $F$ & $\begin{array}{l}p \\
\text { value }\end{array}$ & Power & $\begin{array}{l}\text { WT vs } \\
\text { Het }\end{array}$ & $\begin{array}{l}\text { WT vs } \\
\text { KO }\end{array}$ & $\begin{array}{l}\text { Het vs } \\
\text { KO }\end{array}$ \\
\hline $\begin{array}{l}\text { Time in mouse or object } \\
\text { chamber }\end{array}$ & 2WANOVA & Nonnormal & $528.42 \pm 10.99$ & $509.46 \pm 27.36$ & $501.19 \pm 14.27$ & 0.428 & 0.6540 & 0.116 & 2.557 & 0.116 & 0.348 & 0.131 & 0.878 & 0.069 & - & - & - \\
\hline $\begin{array}{l}\text { Time sniffing mouse or } \\
\text { object }\end{array}$ & 2wANOVA & Normal & $89.02 \pm 6.62$ & $103.43 \pm 10.43$ & $101.27 \pm 11.18$ & 0.670 & 0.5160 & 0.156 & 2.367 & 0.130 & 0.326 & 1.153 & 0.324 & 0.242 & - & - & - \\
\hline $\begin{array}{l}\text { Time close to mouse or } \\
\text { object }\end{array}$ & 2WANOVA & Normal & $162.11 \pm 8.5$ & $168.73 \pm 11.02$ & $146.49 \pm 10.45$ & 1.165 & 0.3200 & 0.244 & 0.888 & 0.351 & 0.152 & 0.037 & 0.964 & 0.055 & - & - & - \\
\hline Male-female social inter & actions, sni & iffing & & & & & & & & & & & & & & & \\
\hline & & & & & & Genotype & & & Cohort & & & $\begin{array}{l}\text { Genoty } \\
\text { cohort }\end{array}$ & pe $\times$ & & $\begin{array}{l}\text { Pairwise } \\
\text { compari }\end{array}$ & $\begin{array}{l}\text { e } \\
\text { risons }\end{array}$ & \\
\hline & Test & $\begin{array}{l}\text { Data } \\
\text { structure }\end{array}$ & WT & Het & KO & $F$ & $\begin{array}{l}p \\
\text { value }\end{array}$ & Power & $F$ & $\begin{array}{l}p \\
\text { value }\end{array}$ & Power & $F$ & $\begin{array}{l}p \\
\text { value }\end{array}$ & Power & $\begin{array}{l}\text { WT vs } \\
\text { Het }\end{array}$ & $\begin{array}{l}\text { WT vs } \\
\text { KO }\end{array}$ & $\begin{array}{l}\text { Het vs } \\
\text { KO }\end{array}$ \\
\hline Anogenital, time (s) & 2WANOVA & Nonnormal & $10.22 \pm 1.62$ & $12.36 \pm 1.86$ & $9.05 \pm 1.43$ & 0.933 & 0.4000 & 0.202 & 0.154 & 0.696 & 0.067 & 0.097 & 0.908 & 0.064 & - & - & - \\
\hline Anogenital, number & 2WANOVA & Nonnormal & $12.56 \pm 3.44$ & $14.28 \pm 4.04$ & $7.77 \pm 2.36$ & 1.049 & 0.3580 & 0.223 & 0.050 & 0.824 & 0.056 & 0.653 & 0.525 & 0.153 & - & - & - \\
\hline $\begin{array}{l}\text { Anogenital, latency } \\
\text { to first (s) }\end{array}$ & 2wANOVA & Nonnormal & $26.91 \pm 11.44$ & $19.61 \pm 8.63$ & $83.18 \pm 25.05$ & 4.238 & 0.0200 & 0.715 & 2.222 & 0.143 & 0.309 & 1.172 & 0.318 & 0.245 & 0.619 & 0.032 & 0.008 \\
\hline Nose to body, time (s) & 2WANOVA & Nonnormal & $14.94 \pm 2.05$ & $14.94 \pm 2.71$ & $16.58 \pm 2.86$ & 0.348 & 0.7080 & 0.103 & 0.783 & 0.381 & 0.140 & 1.722 & 0.190 & 0.344 & - & - & - \\
\hline Nose to body, number & 2WANOVA & Nonnormal & $10.93 \pm 1.54$ & $10.83 \pm 1.68$ & $13.91 \pm 4.35$ & 0.333 & 0.7190 & 0.100 & 0.483 & 0.490 & 0.105 & 2.192 & 0.123 & 0.426 & - & - & - \\
\hline $\begin{array}{l}\text { Nose to body, latency to } \\
\text { first (s) }\end{array}$ & 2WANOVA & Nonnormal & $18.89 \pm 5.38$ & $20.94 \pm 8.22$ & $13.38 \pm 3.97$ & 0.332 & 0.7190 & 0.100 & 1.025 & 0.316 & 0.168 & 0.915 & 0.408 & 0.199 & - & - & - \\
\hline Nose to nose, time (s) & 2WANOVA & Nonnormal & $8.55 \pm 0.85$ & $10.73 \pm 1.07$ & $9.58 \pm 1.13$ & 0.133 & 0.8760 & 0.069 & 0.717 & 0.401 & 0.132 & 1.107 & 0.339 & 0.233 & - & - & - \\
\hline Nose to nose number & 2WANOVA & Nonnormal & $6.31 \pm 0.79$ & $6.91 \pm 0.63$ & $6.61 \pm 1.18$ & 1.118 & 0.3350 & 0.235 & 0.020 & 0.889 & 0.052 & 0.394 & 0.676 & 0.110 & - & - & - \\
\hline $\begin{array}{l}\text { Nose to nose, latency } \\
\text { to first (s) }\end{array}$ & 2WANOVA & Nonnormal & $34.72 \pm 7.85$ & $34.09 \pm 9.24$ & $16.58 \pm 3.97$ & 1.599 & 0.2130 & 0.322 & 0.062 & 0.804 & 0.057 & 0.417 & 0.661 & 0.114 & - & - & - \\
\hline All sniffing, time (s) & 2wANOVA & Nonnormal & $33.77 \pm 3.7$ & $38.05 \pm 4.77$ & $35.23 \pm 4.63$ & 0.155 & 0.8570 & 0.073 & 0.686 & 0.412 & 0.128 & 0.654 & 0.524 & 0.153 & - & - & - \\
\hline All sniffing, number & 2WANOVA & Nonnormal & $29.81 \pm 4.59$ & $32.03 \pm 5.13$ & $28.29 \pm 6.45$ & 0.313 & 0.7330 & 0.097 & 0.138 & 0.712 & 0.065 & 1.522 & 0.229 & 0.308 & - & - & - \\
\hline $\begin{array}{l}\text { All sniffing, latency to } \\
\text { first (s) }\end{array}$ & 2WANOVA & Nonnormal & $5.52 \pm 1.85$ & $4.29 \pm 1.55$ & $7.41 \pm 2.92$ & 0.586 & 0.5610 & 0.142 & 1.267 & 0.266 & 0.197 & 0.042 & 0.959 & 0.056 & - & - & - \\
\hline Male-female social int & actions, ultr & trasonic voce & alization & & & & & & & & & & & & & & \\
\hline & & & & & & Genotype & & & Cohort & & & $\begin{array}{l}\text { Genoty } \\
\text { cohort }\end{array}$ & pe $\times$ & & $\begin{array}{l}\text { Pairwise } \\
\text { compari }\end{array}$ & $\begin{array}{l}\text { e } \\
\text { risons }\end{array}$ & \\
\hline & Test & $\begin{array}{l}\text { Data } \\
\text { structure }\end{array}$ & WT & Het & KO & $F$ & $\begin{array}{l}p \\
\text { value }\end{array}$ & Power & $F$ & $\begin{array}{l}p \\
\text { value }\end{array}$ & Power & $F$ & $\begin{array}{l}p \\
\text { value }\end{array}$ & Power & $\begin{array}{l}\text { WT vs } \\
\text { Het }\end{array}$ & $\begin{array}{l}\text { WT vs } \\
\text { KO }\end{array}$ & $\begin{array}{l}\text { Het } \\
\text { vs KO }\end{array}$ \\
\hline USV, all calls & 2wANOVA & Nonnormal & $380.11 \pm 50.36$ & $378.33 \pm 64.78$ & $287.58 \pm 31.87$ & 1.345 & 0.2704 & 0.276 & 3.242 & 0.078 & 0.422 & 0.193 & 0.825 & 0.078 & - & - & - \\
\hline USV, minute 1 & 2WANOVA & Nonnormal & $84.16 \pm 12.35$ & $94.11 \pm 21.67$ & $64.7 \pm 8.21$ & 1.071 & 0.3507 & 0.227 & 1.180 & 0.283 & 0.186 & 0.865 & 0.428 & 0.190 & - & - & - \\
\hline USV, minute 2 & 2WANOVA & Nonnormal & $68.11 \pm 9.01$ & $73.22 \pm 14.22$ & $57.41 \pm 5.55$ & 0.649 & 0.5271 & 0.152 & 1.150 & 0.289 & 0.183 & 0.070 & 0.932 & 0.060 & - & - & - \\
\hline USV, minute 3 & 2WANOVA & Nonnormal & $77.61 \pm 11.62$ & $68.72 \pm 8.59$ & $57.7 \pm 8.2$ & 1.363 & 0.2659 & 0.279 & 2.155 & 0.149 & 0.301 & 0.799 & 0.456 & 0.178 & - & - & - \\
\hline USV, minute 4 & 2wANOVA & Nonnormal & $74.5 \pm 14.48$ & $76.44 \pm 13$ & $52.7 \pm 4.98$ & 1.566 & 0.2197 & 0.316 & 4.139 & 0.048 & 0.513 & 0.276 & 0.760 & 0.091 & - & - & - \\
\hline USV, minute 5 & 2WANOVA & Nonnormal & $75.72 \pm 14.21$ & $65.83 \pm 11.73$ & $55.05 \pm 8.13$ & 1.092 & 0.3439 & 0.230 & 5.269 & 0.026 & 0.613 & 0.049 & 0.952 & 0.057 & - & - & - \\
\hline USV & Test & $\begin{array}{l}\text { Data } \\
\text { structure }\end{array}$ & & & & $F$ & $\begin{array}{l}p \\
\text { value }\end{array}$ & Power & $\begin{array}{l}\text { WT vs } \\
\text { Het }\end{array}$ & $\begin{array}{l}\text { WT vs } \\
\text { KO }\end{array}$ & $\begin{array}{l}\text { Het vs } \\
\mathrm{KO}\end{array}$ & & & & & & \\
\hline - Time & rMeasures & Sph.viol & & & & 2.964 & 0.0210 & 0.785 & - & - & - & & & & & & \\
\hline - Time $\times$ genotype & rMeasures & Sph.viol & & & & 0.558 & 0.8110 & 0.254 & - & - & - & & & & & & \\
\hline - Genotype & rMeasures & Sph.viol & & & & 1.345 & 0.2704 & 0.276 & - & - & - & & & & & & \\
\hline - Cohort & rMeasures & Sph.viol & & & & 3.242 & 0.0782 & 0.422 & - & - & - & & & & & & \\
\hline - Time $\times$ gen. $\times$ coh . & rMeasures & Sph.viol & & & & 1.245 & 0.2750 & 0.564 & - & - & - & & & & & & \\
\hline - Gen. $\times$ coh. & rMeasures & Sph.viol & & & & 0.193 & 0.8248 & 0.078 & - & - & - & & & & & & \\
\hline
\end{tabular}


Table 8. Continued

\begin{tabular}{|c|c|c|c|c|c|c|c|c|c|c|c|c|c|c|c|c|c|}
\hline \multicolumn{18}{|c|}{ Social transmission of food preference } \\
\hline & \multirow[b]{2}{*}{ Test } & \multirow[b]{2}{*}{$\begin{array}{l}\text { Data } \\
\text { structure }\end{array}$} & \multirow[b]{2}{*}{ WT } & \multirow[b]{2}{*}{ Het } & \multirow[b]{2}{*}{$\mathrm{KO}$} & \multicolumn{3}{|c|}{ Genotype } & \multicolumn{3}{|l|}{ Cohort } & \multicolumn{2}{|c|}{$\begin{array}{l}\text { Genotype } \times \\
\text { cohort }\end{array}$} & \multirow[b]{2}{*}{ Power } & \multicolumn{3}{|c|}{$\begin{array}{l}\text { Pairwise } \\
\text { comparisons }\end{array}$} \\
\hline & & & & & & $F$ & $\begin{array}{l}p \\
\text { value }\end{array}$ & Power & $F$ & $\begin{array}{l}p \\
\text { value }\end{array}$ & Power & $F$ & $\begin{array}{l}p \\
\text { value }\end{array}$ & & $\begin{array}{l}\text { WT vs } \\
\text { Het }\end{array}$ & $\begin{array}{l}\text { WT vs } \\
\text { KO }\end{array}$ & $\begin{array}{l}\text { Het vs } \\
\text { KO }\end{array}$ \\
\hline $\begin{array}{l}\text { Demonstrator sniffing } \\
\text { time }(\mathrm{s})\end{array}$ & 2WANOVA & Nonnormal & $29.8 \pm 6.01$ & $37.44 \pm 6.25$ & $24.69 \pm 6.39$ & 0.756 & 0.4752 & 0.171 & 4.407 & 0.041 & 0.538 & 0.202 & 0.818 & 0.080 & - & - & - \\
\hline Number of sniffing bouts & 2WANOVA & Nonnormal & $9.68 \pm 1.51$ & $13.57 \pm 1.48$ & $7.26 \pm 1.36$ & 4.064 & 0.0236 & 0.695 & 2.772 & 0.103 & 0.371 & 0.099 & 0.906 & 0.064 & 0.126 & 0.733 & 0.021 \\
\hline Time exploring all food (s) & 2WANOVA & Nonnormal & $1533.36 \pm 98.47$ & $1456.68 \pm 98.14$ & $1715.26 \pm 124.97$ & 1.372 & 0.2635 & 0.281 & 0.361 & 0.551 & 0.091 & 0.216 & 0.806 & 0.082 & - & - & - \\
\hline $\begin{array}{l}\text { Time pre-exposed/all } \\
\text { food (\%) }\end{array}$ & 2WANOVA & Nonnormal & $64.89 \pm 3.55$ & $59.21 \pm 4.8$ & $64.54 \pm 4.71$ & 0.589 & 0.5589 & 0.142 & 0.150 & 0.700 & 0.067 & 1.790 & 0.178 & 0.356 & - & - & - \\
\hline Time new/all food (\%) & 2WANOVA & Nonnormal & $35.1 \pm 3.55$ & $40.78 \pm 4.8$ & $35.45 \pm 4.71$ & 0.589 & 0.5589 & 0.142 & 0.150 & 0.700 & 0.067 & 1.790 & 0.178 & 0.356 & - & - & - \\
\hline $\begin{array}{l}\text { Ratio time pre-exposed/ } \\
\text { new }\end{array}$ & 2WANOVA & Nonnormal & $2.6 \pm 0.45$ & $2.31 \pm 0.47$ & $3.06 \pm 0.64$ & 0.636 & 0.5338 & 0.150 & 1.077 & 0.305 & 0.174 & 0.739 & 0.483 & 0.168 & - & - & - \\
\hline $\begin{array}{l}\text { Time spent exploring } \\
\text { cocoa/all food (\%) }\end{array}$ & 2wANOVA & Nonnormal & $51.21 \pm 4.98$ & $50.65 \pm 5.26$ & $50.97 \pm 5.82$ & 0.003 & 0.9969 & 0.050 & 0.240 & 0.626 & 0.077 & 0.856 & 0.431 & 0.188 & - & - & - \\
\hline $\begin{array}{l}\text { Time exploring cinnamon/ } \\
\text { all food (\%) }\end{array}$ & 2wANOVA & Nonnormal & $48.78 \pm 4.98$ & $49.34 \pm 5.26$ & $49.02 \pm 5.82$ & 0.003 & 0.9969 & 0.050 & 0.240 & 0.626 & 0.077 & 0.856 & 0.431 & 0.188 & - & - & - \\
\hline $\begin{array}{l}\text { Ratio time cocoa/ } \\
\text { cinnamon }\end{array}$ & 2WANOVA & Nonnormal & $1.55 \pm 0.31$ & $1.67 \pm 0.4$ & $2.07 \pm 0.64$ & 0.585 & 0.5611 & 0.141 & 0.769 & 0.385 & 0.138 & 0.433 & 0.651 & 0.116 & - & - & - \\
\hline $\begin{array}{l}\text { Total amount of eaten } \\
\text { food }(\mathrm{g})\end{array}$ & 2WANOVA & Nonnormal & $1.21 \pm 0.18$ & $0.82 \pm 0.15$ & $0.58 \pm 0.06$ & 4.286 & 0.0195 & 0.720 & 1.848 & 0.180 & 0.265 & 0.726 & 0.489 & 0.166 & 0.146 & 0.011 & 0.491 \\
\hline $\begin{array}{l}\text { Amount of eaten food, } \\
\text { pre-exposed (g) }\end{array}$ & 2WANOVA & Nonnormal & $0.87 \pm 0.14$ & $0.67 \pm 0.14$ & $0.46 \pm 0.07$ & 2.346 & 0.1068 & 0.452 & 2.802 & 0.101 & 0.375 & 1.058 & 0.355 & 0.224 & - & - & - \\
\hline $\begin{array}{l}\text { Amount of eaten food, } \\
\text { new }(\mathrm{g})\end{array}$ & 2WANOVA & Nonnormal & $0.34 \pm 0.08$ & $0.15 \pm 0.04$ & $0.13 \pm 0.03$ & 4.130 & 0.0223 & 0.703 & 0.063 & 0.803 & 0.057 & 1.108 & 0.339 & 0.233 & 0.065 & 0.048 & 0.990 \\
\hline $\begin{array}{l}\text { Amount of eaten food, } \\
\text { cocoa }(\mathrm{g})\end{array}$ & 2WANOVA & Nonnormal & $0.59 \pm 0.13$ & $0.36 \pm 0.11$ & $0.3 \pm 0.07$ & 1.887 & 0.1629 & 0.373 & 0.343 & 0.561 & 0.089 & 0.720 & 0.492 & 0.165 & - & - & - \\
\hline $\begin{array}{l}\text { Amount of eaten food, } \\
\text { cinnamon }(\mathrm{g})\end{array}$ & 2WANOVA & Nonnormal & $0.62 \pm 0.13$ & $0.46 \pm 0.13$ & $0.3 \pm 0.05$ & 1.563 & 0.2202 & 0.315 & 1.125 & 0.294 & 0.180 & 0.165 & 0.849 & 0.074 & - & - & - \\
\hline $\begin{array}{l}\text { Percentage pre- } \\
\text { exposed vs new }\end{array}$ & Test & $\begin{array}{l}\text { Data } \\
\text { structure }\end{array}$ & & & & All $F$ & $\begin{array}{l}\text { All } p \\
\text { value }\end{array}$ & Power & WT $F$ & $\begin{array}{l}\text { WT } p \\
\text { value }\end{array}$ & Power & Het $F$ & $\begin{array}{l}\text { Het } p \\
\text { value }\end{array}$ & Power & $\mathrm{KO} F$ & $\begin{array}{l}\text { KO } p \\
\text { value }\end{array}$ & Power \\
\hline - Flavor & rMeasures & Sph.ass & & & & 25.686 & 0.0000 & 0.999 & 18.792 & 0.000 & 0.983 & 4.601 & 0.045 & 0.433 & 9.230 & 0.007 & 0.817 \\
\hline - Cohort & rMeasures & Sph.ass & & & & 0.000 & 1.0000 & 0.050 & 0.800 & 0.384 & NA & 0.196 & 0.663 & NA & 0.531 & 0.476 & 0.106 \\
\hline - Flavor $\times$ cohort & rMeasures & Sph.ass & & & & 0.009 & 0.9227 & 0.051 & 6.593 & 0.020 & 0.678 & 0.195 & 0.665 & 0.070 & 1.137 & 0.301 & 0.172 \\
\hline Time cacao vs cinnamon & Test & $\begin{array}{l}\text { Data } \\
\text { structure }\end{array}$ & & & & All $F$ & $\begin{array}{l}\text { All } p \\
\text { value }\end{array}$ & Power & WT $F$ & $\begin{array}{l}\text { WT } p \\
\text { value }\end{array}$ & Power & Het $F$ & $\begin{array}{l}\text { Het } p \\
\text { value }\end{array}$ & Power & $\mathrm{KO} F$ & $\begin{array}{l}\text { KO } p \\
\text { value }\end{array}$ & Power \\
\hline - Flavor & rMeasures & Sph.ass & & & & 0.001 & 0.9745 & 0.050 & 0.058 & 0.812 & 0.050 & 0.035 & 0.854 & 0.054 & 0.058 & 0.812 & 0.063 \\
\hline - Cohort & rMeasures & Sph.ass & & & & 0.100 & 0.7525 & 0.061 & 0.080 & 0.780 & 0.135 & 0.196 & 0.663 & 0.070 & 0.080 & 0.780 & 0.058 \\
\hline - Flavor $\times$ cohort & rMeasures & Sph.ass & & & & 0.001 & 0.9702 & 0.050 & 0.004 & 0.950 & 0.178 & 0.957 & 0.342 & 0.152 & 0.004 & 0.950 & 0.050 \\
\hline $\begin{array}{l}\text { Amount of eaten food, } \\
\text { pre-expose vs new }\end{array}$ & Test & $\begin{array}{l}\text { Data } \\
\text { structure }\end{array}$ & & & & All $F$ & $\begin{array}{l}\text { All } p \\
\text { value }\end{array}$ & Power & WT $F$ & $\begin{array}{l}\text { WT } p \\
\text { value }\end{array}$ & Power & Het $F$ & $\begin{array}{l}\text { Het } p \\
\text { value }\end{array}$ & Power & $\mathrm{KO} F$ & $\begin{array}{l}\text { KO } p \\
\text { value }\end{array}$ & Power \\
\hline - Flavor & rMeasures & Sph.ass & & & & 42.099 & 0.0000 & 1.000 & 13.852 & 0.002 & 0.935 & 13.378 & 0.002 & 0.929 & 13.503 & 0.002 & 0.931 \\
\hline - Cohort & rMeasures & Sph.ass & & & & 2.399 & 0.1276 & 0.330 & 0.400 & 0.537 & 0.091 & 2.323 & 0.147 & 0.299 & 0.131 & 0.722 & 0.063 \\
\hline - Flavor $\times$ cohort & rMeasures & Sph.ass & & & & 3.445 & 0.0692 & 0.445 & 4.346 & 0.055 & 0.496 & 0.872 & 0.364 & 0.142 & 0.080 & 0.781 & 0.058 \\
\hline $\begin{array}{l}\text { Amount of eaten food, } \\
\text { cacao vs cinnamon }\end{array}$ & Test & $\begin{array}{l}\text { Data } \\
\text { structure }\end{array}$ & & & & All $F$ & $\begin{array}{l}\text { All } p \\
\text { value }\end{array}$ & Power & WT $F$ & $\begin{array}{l}\text { WT } p \\
\text { value }\end{array}$ & Power & Het $F$ & $\begin{array}{l}\text { Het } p \\
\text { value }\end{array}$ & Power & $\mathrm{KO} F$ & $\begin{array}{l}\text { KO } p \\
\text { value }\end{array}$ & Power \\
\hline - Flavor & rMeasures & Sph.ass & & & & 0.212 & 0.6473 & 0.074 & 0.005 & 0.945 & 0.050 & 0.265 & 0.614 & 0.077 & 0.011 & 0.918 & 0.051 \\
\hline - Cohort & rMeasures & Sph.ass & & & & 2.399 & 0.1276 & 0.330 & 0.400 & 0.537 & 0.091 & 2.323 & 0.147 & 0.299 & 0.131 & 0.722 & 0.063 \\
\hline - Flavor $\times$ cohort & rMeasures & Sph.ass & & & & 0.117 & 0.7342 & 0.063 & 0.178 & 0.679 & 0.068 & 0.052 & 0.822 & 0.055 & 0.271 & 0.610 & 0.078 \\
\hline
\end{tabular}

WT, wild-type mice; Het, heterozygous mice; KO, homozygous knock-out mice. C, center chamber; M, mouse chamber; O, object chamber. Group values are reported as mean \pm SEM. Bold font indicates significant results $(p<0.05)$. Individual results and statistical analyses for cohorts 1 and 2 are available in Extended Data Table 8-1. 2wANOVA: 2-way ANOVA, rMeasures: repeated measures, Sph.ass: sphericity assumed, Sph.viol: sphericity violated, gen: genotype, coh: cohort.

Individuals with ASD can maintain rigid habits and frequently show strong insistence on sameness and upset by changes in routine. To examine this domain, Shank $3^{\Delta 4-22}$ mice were trained for $4 \mathrm{~d}$ in the Barnes maze, a test of spatial learning and memory, until all the mice were able to quickly locate an escape box hidden under one of the target locations, then the location of the escape box was moved and mice were tested for reversal learning for four additional days. During the initial learning, all the genotypes were able to find the escape hatch equally well, although
Shank $3^{\Delta 4-22}$ homozygous mice took $1 \mathrm{~d}$ longer to reach criteria (Fig. $8 \mathrm{C}$, left panel). All genotypes preferred the correct quadrant in the first probe test ran immediately after the initial training (Fig. $8 \mathrm{C}$, middle panel). When the escape hatch was moved to the opposite side of the maze, both Shank $3^{\Delta 4-22}$ wild-type and heterozygotes immediately learned the new position, while a 1-d delay was, once again, observed for the Shank $3^{\Delta 4-22}$ homozygous mice. Genotypes differed markedly in the second probe test, however; while wild-type mice spent most

continued

displayed normal habituation/dishabituation for social scents (unfamiliar male and female bedding). The olfactory habituation and dishabituation to nonsocial and social odors was measured as cumulative time spent sniffing a sequence of identical and novel odors delivered on cotton swabs inserted into a clean cage. WT, wild-type mice; Het, heterozygous mice; KO, homozygous knock-out mice. $* p<0.05, * * p<0.1, * * * p<0.001$. 
A

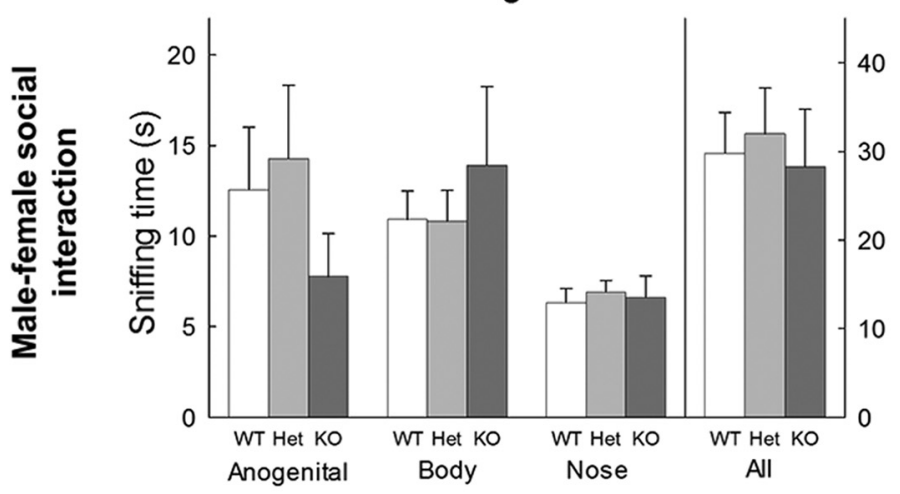

Sniffing latency

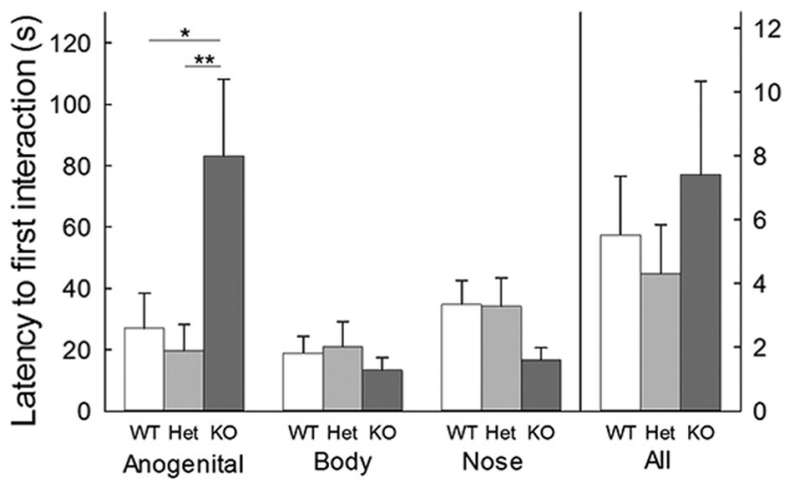

B

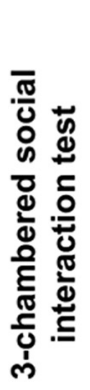

Sociability

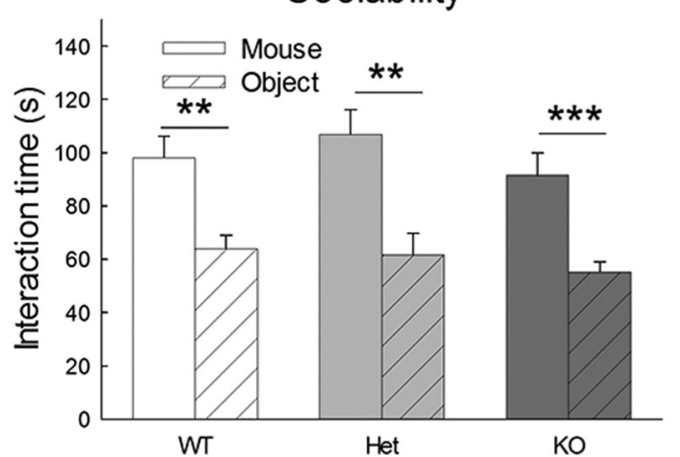

C

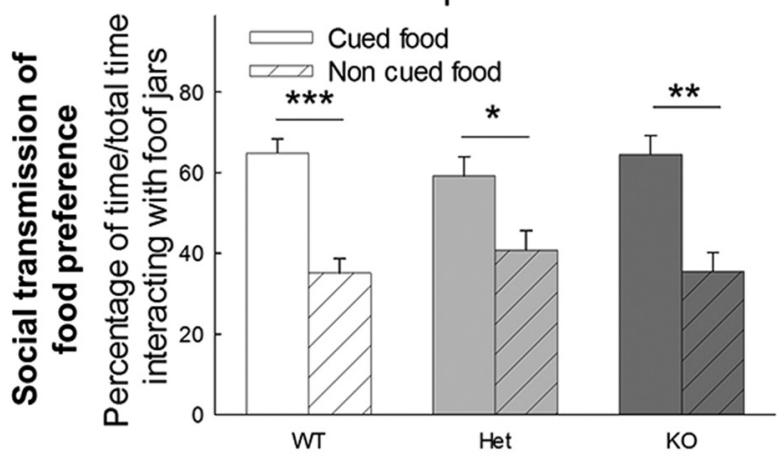

Figure 5. Social behavior of Shank $3^{\Delta 4-22}$-deficient mice. $\boldsymbol{A}$, Male social interaction in response to the presentation of an unfamiliar conspecific female in estrus and scored by the cumulative sniffing time and latency from the male toward different body regions of the female. No genotype differences were evident in the dyadic male-female social interaction for the overall sniffing time from the male toward the female, however a trend toward a decrease in anogenital sniffing as well as a significant increase of the latency to initiate the first anogenital sniffing event was observed in Shank $3^{\Delta 4-22}$ homozygous mice. B, Preference for social stimulus in the three-chambered social interaction test measured by cumulative time interacting with either a mouse or an inanimate object. All three genotypes demonstrated a significant preference for an unfamiliar mouse over a nonsocial object. $\boldsymbol{C}$, Social transmission of food preference measured by the time spent by the test mouse sniffing the demonstrator mouse and the time spent interacting with both cued and noncued food. All genotypes had a strong preference for the food flavor presented by the demonstrator mouse. USVs and time spent sniffing the demonstrator during the demonstrator interaction phase are displayed in Extended Data Figure 5-1. WT, wild-type mice; Het, heterozygous mice; KO, homozygous knock-out mice. $* p<0.05, * * p<0.1, * * * p<0.001$.

time in the new target quadrant, Shank $3^{\Delta 4-22}$ heterozygous mice split their time $75 / 25 \%$ between new and old targets, whereas Shank3 $3^{\Delta 4-22}$ homozygous animals spent equal time in both targets (Fig. $8 C$, right panel). This impaired reversal learning implies that Shank3 deficiency increases susceptibility to proactive interference where learning of a previous rule interferes with the new rule.

\section{Learning and memory in Shank $3^{\Delta 4-22}$-deficient mice}

In addition to the Barnes maze, animals were tested in two additional learning and memory tests, specifically, the Y-maze spontaneous alternation test and the fear conditioning test. Detailed results are reported in Table 12.

When looking at the spontaneous alternation behavior in the Y-maze, no differences were observed between the genotypes in any of the background strains regarding either the total number of choices, the percentage of correct choices or the percentage of errors (Fig. 9A).
Moreover, no arm preference was seen for any of the groups.

In the training session of the fear conditioning test, minimal levels of freezing behavior were seen for all the genotypes during the 5-min habituation period; however, while this percentage of spontaneous freezing decreased before the presentations of cue-shock pairings for the Shank $3^{\Delta 4-22}$ wild-type and heterozygotes, it remained at significantly higher level for Shank $3^{\Delta 4-22}$ homozygous mice. A significant genotype effect was then found during the training session in postshock freezing, with Shank $3^{\Delta 4-22}$ homozygous mice displaying higher levels of freezing compared with wild-type and heterozygous mice (Fig. 9B, left panel). The opposite was observed during contextual recall where even if all the mice freeze significantly more than during the habituation of the training sessions a trend toward a decrease (significant during the first minute) of freezing was observed for Shank $3^{\Delta 4-22}$ homozygous mice compared to wild-type or heterozygous littermates (Fig. 9B, middle 
Table 9. Detailed results and statistical analyses related to the avoidance behavior

Novel object habituation

$\begin{array}{lll} & \text { Test } & \begin{array}{l}\text { Data } \\ \text { structure }\end{array} \\ \text { Total distance }(\mathrm{cm}) & \text { 2wANOVAA } & \text { Nonnorma } \\ \text { Time in left side }(\mathrm{s}) & \text { 2wANOVA } & \text { Normal } \\ \text { Time in right side }(\mathrm{s}) & \text { 2wANOVA } & \text { Normal } \\ & \text { Test } & \begin{array}{l}\text { Data } \\ \text { structure }\end{array} \\ \begin{array}{l}\text { Time spend in left } \\ \text { vs right half side }\end{array} & \text { rMeasures } & \begin{array}{l}\text { Sph.ass } \\ \text { - Side }\end{array} \\ \text { - Cohort } & \text { rMeasures } & \text { Sph.ass } \\ \text { - Side } \times \text { cohort } & \text { rMeasures } & \text { Sph.ass }\end{array}$

Novel object recognition: training with two identical objects

\begin{tabular}{|c|c|c|}
\hline & Test & $\begin{array}{l}\text { Data } \\
\text { structure }\end{array}$ \\
\hline Total distance $(\mathrm{cm})$ & 2WANOVA & Nonnormal \\
\hline Time in left side (s) & 2WANOVA & Normal \\
\hline Time in right side (s) & 2WANOVA & Normal \\
\hline Number of side switches & 2WANOVA & Normal \\
\hline $\begin{array}{l}\text { Number of left object } \\
\text { exploration }\end{array}$ & 2WANOVA & Nonnormal \\
\hline $\begin{array}{l}\text { Number of right object } \\
\text { exploration }\end{array}$ & 2WANOVA & Nonnorma \\
\hline $\begin{array}{l}\text { Time exploring left } \\
\text { object (s) }\end{array}$ & 2WANOVA & Nonnorma \\
\hline $\begin{array}{l}\text { Time exploring right } \\
\text { object (s) }\end{array}$ & 2WANOVA & Nonnorma \\
\hline Latency to left object (s) & 2WANOVA & Nonnormal \\
\hline Latency to right object (s) & 2WANOVA & Nonnormal \\
\hline $\begin{array}{l}\text { Total number of object } \\
\text { exploration }\end{array}$ & 2WANOVA & Nonnormal \\
\hline $\begin{array}{l}\text { Total time exploring } \\
\text { objects (s) }\end{array}$ & 2WANOVA & Nonnorm \\
\hline Latency to any object (s) & 2wANOVA & Nonnorma \\
\hline $\begin{array}{l}\text { Sniffing time,left vs } \\
\text { right (s) }\end{array}$ & Test & $\begin{array}{l}\text { Data } \\
\text { structure }\end{array}$ \\
\hline - Side & rMeasures & Sph.ass \\
\hline - Cohort & rMeasures & Sph.ass \\
\hline - Side $\times$ cohort & rMeasures & Sph.ass \\
\hline $\begin{array}{l}\text { Number of interactions, } \\
\text { left vs right }\end{array}$ & Test & $\begin{array}{l}\text { Data } \\
\text { structure }\end{array}$ \\
\hline - Side & rMeasures & Sph.ass \\
\hline - Cohort & rMeasures & Sph.ass \\
\hline - Side $\times$ cohort & rMeasures & Sph.ass \\
\hline $\begin{array}{l}\text { Time in left vs } \\
\text { right halves (s) }\end{array}$ & Test & $\begin{array}{l}\text { Data } \\
\text { structure }\end{array}$ \\
\hline - Side & rMeasures & Sph.ass \\
\hline - Cohort & rMeasures & Sph.ass \\
\hline - Side $\times$ cohort & rMeasures & Sph.ass \\
\hline
\end{tabular}

Novel object recognition: test with one new object

Total distance $(\mathrm{cm})$ Time in new object side (s) Time pre-exposed object side $(s)$

Number of side switches

Number of new object exploration

Number of pre-exposed object exploration

Time exploring new object (s)

Time exploring pre-exposed 2wANOVA Nonnormal 12.49 \pm 2.05 object (s)

Latency new object (s)

Latency to pre-exposed object (s)

Total number of object exploration

Total time exploring objects (s)

Novel object, latency to observe any object (s)

2WANOVA Normal $14.57 \pm 0.77$

2 WANOVA Nonnormal $22.73 \pm 2.66$

2WANOVA Nonnormal $18.52 \pm 4.35$

2WANOVA Nonnormal $27.84 \pm 3.58$

2WANOVA Nonnormal $16.68 \pm 5.17$

2WANOVA Nonnormal $30.73 \pm 8.99$

2WANOVA Nonnormal $41.26 \pm 6.59$

2WANOVA Nonnormal $40.33 \pm 5.04$

2wANOVA Nonnormal $14.17 \pm 4.79$
Het

$421.86 \pm 196.942175 \pm 173.66$ $50.86+9.32 \quad 148.91+10.25$ $48.86 \pm 9.35$
48.96 $15.94 \pm 1.28$

$148.91 \pm 10.25$
$150.82+10.23$ $150.82 \pm 10.23$
$14.26 \pm 1$ $19.52 \pm 2.54$

$28.84 \pm 7.87$

$19.89 \pm 3.1$

$23.94 \pm 4.06$

$21.74 \pm 7.62$

$13.99 \pm 5.42$

$48.36 \pm 9.86$

$43.83 \pm 6.59$

$13.43 \pm 5.8$

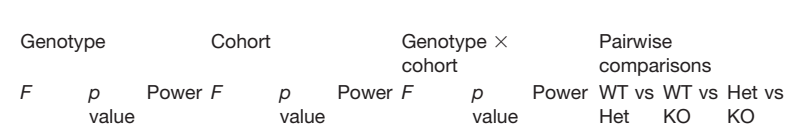

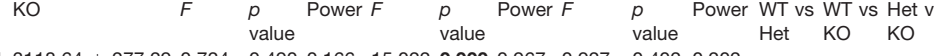

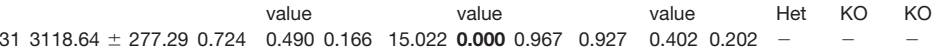
$\begin{array}{lllllllllll}322.76 & \pm 23.1 & 1.086 & 0.345 & 0.230 & 0.112 & 0.739 & 0.062 & 2.009 & 0.145 & 0.396\end{array}$

All $F \quad$ All $p$ Power WT $F \quad$ WT $p$ Power Het $F$ Het $p$ Power KO $F \quad$ KO $p$ Power value value value value $\begin{array}{llllllllllll}0.001 & 0.979 & 0.052 & 0.445 & 0.514 & 0.097 & 0.145 & 0.708 & 0.065 & 1.249 & 0.279 & 0.184\end{array}$

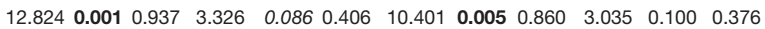
$\begin{array}{llllllllllll}0.044 & 0.835 & 0.062 & 0.096 & 0.761 & 0.060 & 1.352 & 0.261 & 0.195 & 2.506 & 0.132 & 0.321\end{array}$

Genotype Cohort $\quad$ Genotype $\times \quad$ Pairwise

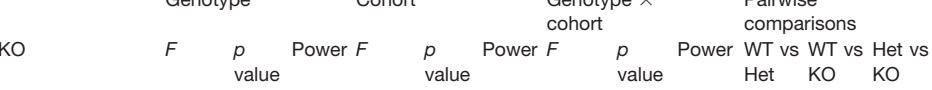

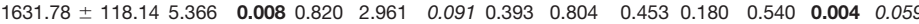
$\begin{array}{llllllllllll}176.59 & \pm 16.36 & 1.489 & 0.235 & 0.303 & 0.040 & 0.843 & 0.054 & 0.905 & 0.411 & 0.198 & -\end{array}$

$\begin{array}{llllllllllll}122.95 & \pm 16.33 & 1.511 & 0.230 & 0.307 & 0.042 & 0.839 & 0.055 & 0.896 & 0.414 & 0.196 & -\end{array}$

$\begin{array}{lllllllllll}13.89 \pm 1.21 & 0.822 & 0.445 & 0.183 & 0.259 & 0.613 & 0.079 & 0.459 & 0.635 & 0.121\end{array}$

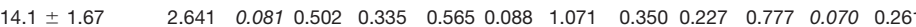

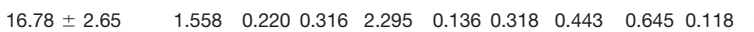

$10.67 \pm 2.11$

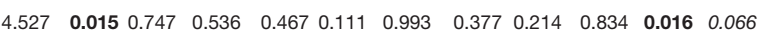

$11.28 \pm 2.37$

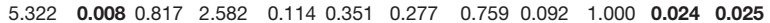

$20.67 \pm 3.94$

$17.92 \pm 3.01$

$30.89 \pm 4.09$

$\begin{array}{lllllllll}0.353 & 0.704 & 0.104 & 0.068 & 0.796 & 0.057 & 0.138 & 0.872 & 0.070\end{array}$

$\begin{array}{llllllllll}0.348 & 0.708 & 0.103 & 3.325 & 0.074 & 0.432 & 0.816 & 0.448 & 0.182\end{array}$

$\begin{array}{llllllllll}1.992 & 0.147 & 0.393 & 0.959 & 0.332 & 0.161 & 0.596 & 0.555 & 0.144\end{array}$

$21.95 \pm 4.26$

$\begin{array}{llllllllllll}5.733 & 0.006 & 0.846 & 1.680 & 0.201 & 0.246 & 0.041 & 0.960 & 0.056 & 0.954 & 0.011 & 0.024\end{array}$

$\begin{array}{lllllllll}0.674 & 0.514 & 0.157 & 1.455 & 0.233 & 0.220 & 0.482 & 0.621 & 0.125\end{array}$

All $F \quad$ All $p \quad$ Power WT $F \quad$ WT $p$ Power Het $F$ Het $p$ Power KO $F$ KO $p$ Power value

value

value

value

$\begin{array}{llllllllllll}2.505 & 0.119 & 0.343 & 1.375 & 0.257 & 0.198 & 1.701 & 0.209 & 0.234 & 0.128 & 0.725 & 0.063\end{array}$ $\begin{array}{llllllllllll}0.943 & 0.336 & 0.159 & 0.245 & 0.627 & 0.075 & 0.607 & 0.447 & 0.114 & 1.226 & 0.284 & 0.182\end{array}$ $\begin{array}{llllllllllll}1.598 & 0.211 & 0.237 & 7.358 & 0.015 & 0.725 & 0.829 & 0.375 & 0.138 & 1.756 & 0.203 & 0.240\end{array}$

All $F \quad$ All $p$ Power WT $F \quad$ WT $p$ Power Het $F$ Het $p$ Power KO $F$ KO $p$ Power value value value

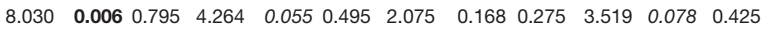
$\begin{array}{llllllllllll}1.277 & 0.263 & 0.199 & 1.149 & 0.299 & 0.173 & 0.375 & 0.548 & 0.089 & 0.311 & 0.585 & 0.082\end{array}$

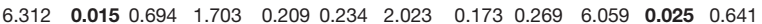
$\begin{array}{lllll}\text { All } F \quad \begin{array}{ll}\text { All } p \\ \text { value }\end{array} & \text { Power WT } F \quad \text { WT } p \text { Power Het } F & \begin{array}{l}\text { Het } p \\ \text { value }\end{array} & \text { Power KO } F \text { KO } p & \text { Power } \\ \text { value } & \end{array}$

$\begin{array}{llllllllllll}1.555 & 0.218 & 0.232 & 0.000 & 0.994 & 0.050 & 0.013 & 0.909 & 0.051 & 2.506 & 0.132 & 0.321\end{array}$ $\begin{array}{llllllllllll}0.591 & 0.445 & 0.117 & 0.142 & 0.711 & 0.065 & 3.906 & 0.065 & 0.462 & 0.161 & 0.693 & 0.067\end{array}$ $\begin{array}{llllllllllll}0.110 & 0.742 & 0.062 & 0.371 & 0.551 & 0.089 & 0.224 & 0.642 & 0.073 & 0.856 & 0.368 & 0.141\end{array}$

Genotype Cohort Genotype $\times$ cohort Pairwise

KO $\quad F \quad p_{\text {value }}$ Power $F \quad p_{\text {value }} \quad$ Power $F \quad p_{\text {value }} \quad \begin{aligned} & \text { Power WT vs WT vs Het vs } \\ & \text { Het KO KO }\end{aligned}$

Het KO

$\begin{array}{llllllllllll}0.000 & 0.968 & 0.000 & 0.989 & 0.050 & 1.066 & 0.352 & 0.227 & 0.059 & 0.000 & 0.117\end{array}$ $\begin{array}{llllllllllllllllll}2 \text { WANOVA } & \text { Normal } & 1973.56 \pm 156.94 & 1482.1 \pm 167.46 & 1057.83 \pm 110.93 & 9.082 & 0.000 & 0.968 & 0.000 & 0.989 & 0.050 & 1.066 & 0.352 & 0.227 & 0.059 & 0.000 & 0.117 \\ \text { 2wANOVA } & \text { Normal } & 151.59 \pm 9.78 & 143.64 \pm 15.18 & 174.14 \pm 19.84 & 0.985 & 0.380 & 0.212 & 0.004 & 0.947 & 0.050 & 0.545 & 0.583 & 0.135 & - & - & -\end{array}$ 2WANOVA Normal $147.79 \pm 9.8 \quad 155.86 \pm 15.26$

$9.84 \pm 1.32$ $125.22 \pm 19.97$

$17.84 \pm 2.68$

$8.52 \pm 1.05$

$13.05 \pm 2.92$

$6.26 \pm 0.93$

$\begin{array}{llllllllllll}8.853 & 0.001 & 0.964 & 0.422 & 0.519 & 0.098 & 1.128 & 0.332 & 0.238 & 0.009 & 0.001 & 0.666\end{array}$ $\begin{array}{llllllllllll}14.115 & 0.000 & 0.998 & 1.316 & 0.257 & 0.203 & 0.241 & 0.787 & 0.086 & 0.289 & 0.000 & 0.002\end{array}$

$21.74 \pm 2.89 \quad 6.04 \pm 1.39$

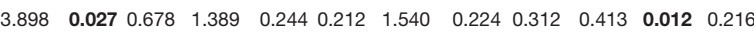

$12.03 \pm 2.55 \quad 3.8 \pm 0.78$

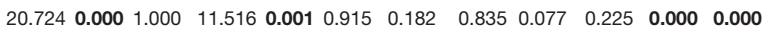

$50.97 \pm 18.18$

$49.62 \pm 18.45$

$72.65 \pm 14.88$ $\begin{array}{llllllllllll}6.051 & 0.004 & 0.866 & 0.047 & 0.829 & 0.055 & 0.351 & 0.706 & 0.103 & 0.985 & 0.009 & 0.014\end{array}$

$30.89 \pm 5.37$

$\begin{array}{llllllllllll}3.295 & 0.045 & 0.600 & 1.589 & 0.213 & 0.236 & 0.299 & 0.743 & 0.095 & 0.193 & 0.043 & 0.753\end{array}$ $\begin{array}{llllllllll}1.728 & 0.188 & 0.346 & 0.164 & 0.687 & 0.068 & 0.245 & 0.783 & 0.087\end{array}$

$33.77 \pm 4.55$

$12.05 \pm 1.53$

$\begin{array}{llllllllllll}8.267 & 0.001 & 0.952 & 0.036 & 0.850 & 0.054 & 0.920 & 0.405 & 0.200 & 0.321 & 0.000 & 0.029\end{array}$

$16.53 \pm 7.27$
$9.85 \pm 1.88$

$41.36 \pm 11.68$

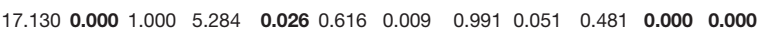

(Continued) 
Table 9. Continued

\begin{tabular}{|c|c|c|c|c|c|c|c|c|c|c|c|c|c|c|c|c|c|}
\hline $\begin{array}{l}\text { Sniffing time, new vs } \\
\text { pre-exposed object }\end{array}$ & Test & $\begin{array}{l}\text { Data } \\
\text { structure }\end{array}$ & & & & All $F$ & $\begin{array}{l}\text { All } p \\
\text { value }\end{array}$ & Power & WT $F$ & $\begin{array}{l}\text { WT } p \\
\text { value }\end{array}$ & Power & Het $F$ & $\begin{array}{l}\text { Het } p \\
\text { value }\end{array}$ & Power & KO F & $\begin{array}{l}\text { KO } p \\
\text { value }\end{array}$ & Power \\
\hline - Side & rMeasures & Sph.ass & & & & 37.818 & 0.000 & 1.000 & 37.629 & 0.000 & 1.000 & 13.312 & 0.002 & 0.930 & 3.302 & 0.087 & 0.403 \\
\hline - Cohort & rMeasures & Sph.ass & & & & 2.146 & 0.149 & 0.302 & 1.221 & 0.285 & 0.181 & 1.164 & 0.296 & 0.175 & 14.732 & 0.001 & 0.951 \\
\hline - Side $\times$ cohort & rMeasures & Sph.ass & & & & 8.100 & 0.006 & 0.798 & 5.648 & 0.029 & 0.611 & 4.628 & 0.046 & 0.528 & 3.660 & 0.073 & 0.439 \\
\hline $\begin{array}{l}\text { Number of interactions, new } \\
\text { vs pre-exposed }\end{array}$ & Test & $\begin{array}{l}\text { Data } \\
\text { structure }\end{array}$ & & & & All $F$ & $\begin{array}{l}\text { All } p \\
\text { value }\end{array}$ & Power & WT $F$ & $\begin{array}{l}\text { WT } p \\
\text { value }\end{array}$ & Power & Het $F$ & $\begin{array}{l}\text { Het } p \\
\text { value }\end{array}$ & Power & $\mathrm{KO} F$ & $\begin{array}{l}\text { KO } p \\
\text { value }\end{array}$ & Power \\
\hline - Side & rMeasures & Sph.ass & & & & 9.499 & 0.003 & 0.857 & 3.824 & 0.067 & 0.454 & 11.442 & 0.004 & 0.890 & 0.314 & 0.582 & 0.083 \\
\hline - Cohor & rMeasures & Sph.ass & & & & 0.221 & 0.640 & 0.075 & 0.567 & 0.462 & 0.110 & 0.045 & 0.835 & 0.055 & 16.708 & 0.001 & 0.970 \\
\hline - Side $\times$ cohort & rMeasures & Sph.ass & & & & 9.882 & 0.003 & 0.870 & 5.751 & 0.028 & 0.618 & 5.258 & 0.035 & 0.580 & 1.686 & 0.211 & 0.232 \\
\hline $\begin{array}{l}\text { Time in new vs pre- } \\
\text { exposed halves (s) }\end{array}$ & Test & $\begin{array}{l}\text { Data } \\
\text { structure }\end{array}$ & & & & All $F$ & $\begin{array}{l}\text { All } p \\
\text { value }\end{array}$ & Power & WT $F$ & $\begin{array}{l}\text { WT } p \\
\text { value }\end{array}$ & Power & Het $F$ & $\begin{array}{l}\text { Het } p \\
\text { value }\end{array}$ & Power & $\mathrm{KO} F$ & $\begin{array}{l}\text { KO } p \\
\text { value }\end{array}$ & Power \\
\hline - Side & rMeasures & Sph.ass & & & & 0.565 & 0.456 & 0.114 & 0.029 & 0.867 & 0.053 & 0.185 & 0.673 & 0.069 & 1.375 & 0.257 & 0.198 \\
\hline - Cohort & rMeasures & Sph.ass & & & & 5.062 & 0.028 & 0.599 & 2.758 & 0.115 & 0.347 & 2.527 & 0.130 & 0.323 & 1.348 & 0.262 & 0.195 \\
\hline - Side $\times$ cohort & rMeasures & Sph.ass & & & & 0.020 & 0.889 & 0.052 & 0.010 & 0.920 & 0.051 & 0.446 & 0.513 & 0.097 & 0.397 & 0.537 & 0.091 \\
\hline \multicolumn{18}{|l|}{ Marble burying } \\
\hline & & & & & & \multicolumn{3}{|c|}{ Genotype } & \multicolumn{3}{|l|}{ Cohort } & \multicolumn{3}{|c|}{$\begin{array}{l}\text { Genotype } \times \\
\text { cohort }\end{array}$} & \multicolumn{3}{|c|}{$\begin{array}{l}\text { Pairwise } \\
\text { comparisons }\end{array}$} \\
\hline & Test & $\begin{array}{l}\text { Data } \\
\text { structure }\end{array}$ & WT & Het & $\mathrm{KO}$ & $F$ & $\begin{array}{l}p \\
\text { value }\end{array}$ & Power & $F$ & $\begin{array}{l}p \\
\text { value }\end{array}$ & Power & $F$ & $\begin{array}{l}p \\
\text { value }\end{array}$ & Power & $\begin{array}{l}\text { WT vs } \\
\text { Het }\end{array}$ & $\begin{array}{l}\text { WT vs } \\
\text { KO }\end{array}$ & $\begin{array}{l}\text { Het vs } \\
\text { KO }\end{array}$ \\
\hline Number of burried marbles & 2WANOVA & Nonnormal & $13.63 \pm 1.29$ & $13.78 \pm 1$ & $3.77 \pm 1.07$ & 18.723 & 0.000 & 1.000 & 0.069 & 0.793 & 0.217 & 0.370 & 0.693 & 0.051 & 0.995 & 0.000 & 0.000 \\
\hline \multicolumn{18}{|c|}{ Repetitive novel object contact task, exploration } \\
\hline & & & & & & \multicolumn{3}{|c|}{ Genotype } & \multicolumn{3}{|l|}{ Cohort } & \multicolumn{3}{|c|}{ Genotype $\times$ cohort } & \multicolumn{3}{|c|}{$\begin{array}{l}\text { Pairwise } \\
\text { comparisons }\end{array}$} \\
\hline & Test & $\begin{array}{l}\text { Data } \\
\text { structure }\end{array}$ & WT & Het & $\mathrm{KO}$ & $F$ & $\begin{array}{l}p \\
\text { value }\end{array}$ & Power & $F$ & $\begin{array}{l}p \\
\text { value }\end{array}$ & Power & $F$ & $\begin{array}{l}p \\
\text { value }\end{array}$ & Power & $\begin{array}{l}\text { WT vs } \\
\text { Het }\end{array}$ & $\begin{array}{l}\text { WT vs } \\
\text { KO }\end{array}$ & $\begin{array}{l}\text { Het vs } \\
\text { KO }\end{array}$ \\
\hline $\begin{array}{l}\text { Time exploring all the } \\
\text { objects }\end{array}$ & 2WANOVA & Nonnormal & $82.08 \pm 11.28$ & $84.8 \pm 7$ & $53.37 \pm 5.01$ & 7.964 & 0.001 & 0.943 & 24.654 & 0.000 & 0.998 & 0.647 & 0.528 & 0.152 & 0.956 & 0.014 & 0.006 \\
\hline $\begin{array}{l}\text { Total number of object } \\
\text { interactions }\end{array}$ & 2WANOVA & Normal & $83.27 \pm 7.92$ & $94.21 \pm 6.22$ & $83.47 \pm 6.4$ & 2.108 & 0.133 & 0.412 & 73.475 & 0.000 & 1.000 & 1.110 & 0.338 & 0.234 & - & - & - \\
\hline \multicolumn{18}{|l|}{ Nest building } \\
\hline & & & & & & \multicolumn{3}{|c|}{ Genotype } & \multicolumn{3}{|l|}{ Cohort } & \multicolumn{3}{|c|}{ Genotype $\times$ cohort } & \multicolumn{3}{|c|}{$\begin{array}{l}\text { Pairwise } \\
\text { comparisons }\end{array}$} \\
\hline & Test & $\begin{array}{l}\text { Data } \\
\text { structure }\end{array}$ & WT & Het & $\mathrm{KO}$ & $F$ & $\begin{array}{l}p \\
\text { value }\end{array}$ & Power & $F$ & $\begin{array}{l}p \\
\text { value }\end{array}$ & Power & $F$ & $\begin{array}{l}p \\
\text { value }\end{array}$ & Power & $\begin{array}{l}\text { WT vs } \\
\text { Het }\end{array}$ & $\begin{array}{l}\text { WT vs } \\
\text { KO }\end{array}$ & $\begin{array}{l}\text { Het vs } \\
\text { KO }\end{array}$ \\
\hline Nest shredded & 2WANOVA & Nonnormal & $1.84 \pm 0.08$ & $1.94 \pm 0.05$ & $1.36 \pm 0.15$ & 7.785 & 0.001 & 0.939 & 2.814 & 0.100 & 0.377 & 0.364 & 0.697 & 0.105 & 0.455 & 0.005 & 0.000 \\
\hline Nest dispersion & 2WANOVA & Nonnormal & $1.94 \pm 0.05$ & $1.94 \pm 0.05$ & $1.73 \pm 0.14$ & 1.580 & 0.216 & 0.320 & 0.919 & 0.342 & 0.156 & 3.425 & 0.040 & 0.618 & - & - & - \\
\hline Nest density & 2wANOVA & Nonnormal & $1.26 \pm 0.14$ & $0.73 \pm 0.16$ & $0.73 \pm 0.21$ & 2.726 & 0.075 & 0.515 & 0.393 & 0.534 & 0.094 & 0.266 & 0.768 & 0.090 & 0.050 & 0.046 & 0.966 \\
\hline Nest shape & 2WANOVA & Nonnormal & $2.57 \pm 0.19$ & $2.05 \pm 0.23$ & $1.36 \pm 0.27$ & 5.851 & 0.005 & 0.854 & 3.065 & 0.086 & 0.404 & 0.097 & 0.908 & 0.064 & 0.153 & 0.001 & 0.055 \\
\hline Nest walls & 2WANOVA & Nonnormal & $1.21 \pm 0.14$ & $1 \pm 0.18$ & $0.42 \pm 0.17$ & 5.649 & 0.006 & 0.840 & 0.097 & 0.756 & 0.061 & 0.833 & 0.440 & 0.185 & 0.359 & 0.002 & 0.023 \\
\hline Nest total score & 2WANOVA & Nonnormal & $8.84 \pm 0.45$ & $7.68 \pm 0.51$ & $5.63 \pm 0.73$ & 7.223 & 0.002 & 0.921 & 1.121 & 0.295 & 0.180 & 0.020 & 0.980 & 0.053 & 0.184 & 0.000 & 0.019 \\
\hline
\end{tabular}

WT, wild-type mice; Het, heterozygous mice; KO, homozygous knock-out mice. Group values are reported as mean \pm SEM. Bold font indicates significant results $(p<0.05)$. Individual results and statistical analyses for cohorts 1 and 2 are available in Extended Data Table 9-1. 2wANOVA: 2-way ANOVA, rMeasures: repeated measures, Sph.ass: sphericity assumed, gen: genotype, coh: cohort.

panel). An increase of freezing was seen in both during and after the cue presentation (trend for the first cue, significant during and after the second cue) Shank $3^{\Delta 4-22}$ homozygous mice (Fig. 9B, right panel).

\section{Anxiety-related behaviors in Shank $3^{\Delta 4-22}$-deficient mice}

Anxiety-like behaviors were monitored in the open field and in the elevated zero-maze, and detailed results are displayed in Table 13.

No significant difference between the genotypes was observed in the open field thigmotaxis level (Fig. 10A), but a decrease in the total number of times the mice reared (mainly driven by against wall rears) was observed in the Shank $3^{\Delta 4-22}$ homozygous animals (Fig. 10B). No significant effects of an interaction between the time and genotype were observed for any of the parameters.

In the elevated zero-maze, all animals showed a preference for the closed arcs versus the open arcs; however, Shank $3^{\Delta 4-22}$ homozygotes spent less time in the open arcs than their wild-type and heterozygous littermates. Similarly, a significant decrease of the duration of head dipping exploratory behavior in the open arcs was seen in those animals (Fig. 10B). No genotype differences were seen for other parameters.

This indicates increases in anxiety in the Shank $3^{\Delta 4-22}$ homozygotes. In support of this, the long-lasting spontaneous freezing observed in Shank $3^{\Delta 4-22}$ homozygous animals during the habituation and before the soundshock association in the fear conditioning training (Fig. $9 B$ ) could also be explained by a higher anxiety level those animals.

\section{Discussion}

Given the prevalence of complete SHANK3 deletions in PMS, we generated Shank $3^{\Delta 4-22}$ mice by targeting exons $4-22$, thereby disrupting all isoforms and providing improved construct validity compared to previously reported models. We conducted an extensive behavioral phenotyping of neonatal (P0-P21) and adult (three to eight months) mice to address both core symptoms and comorbidities observed in PMS. We confirmed our prediction that Shank $3^{\Delta 4-22}$ mice homozygous and in some instances heterozygous mice have a more severe phenotype than previously published models with partial deletions of Shank3 (summarized in Fig. 11). Our findings are 
A

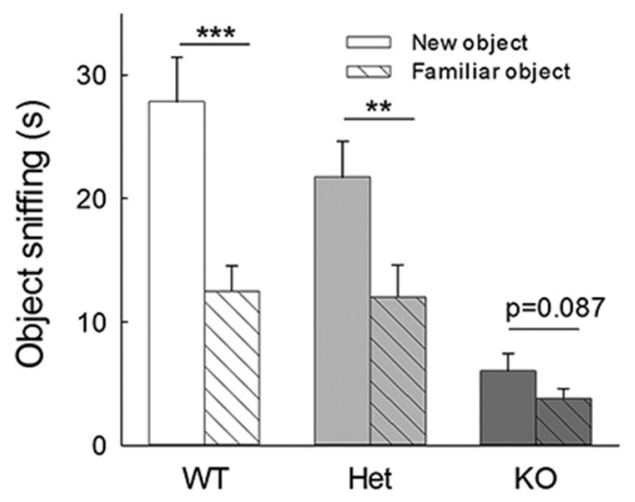

Novel object exploration
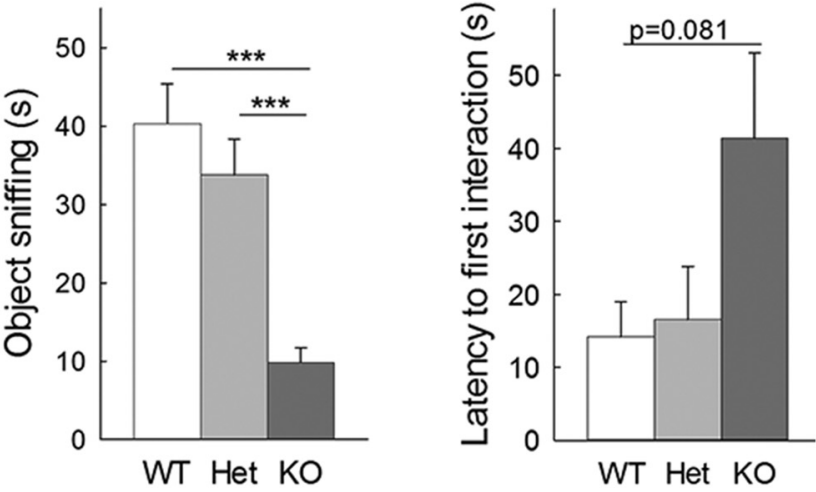
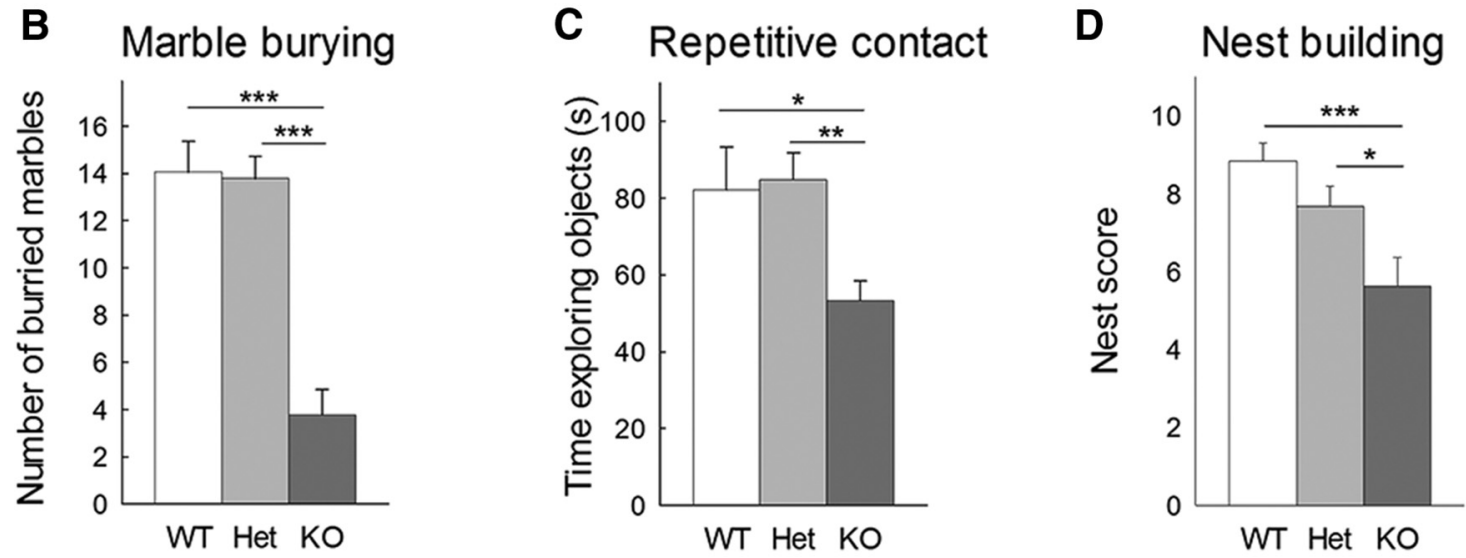

Olfactory habituation/dishabituation - All interactions

E

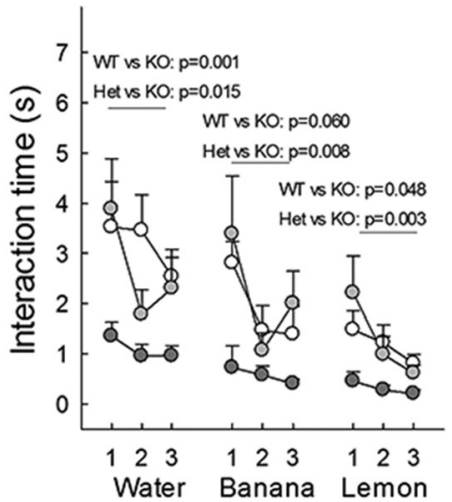

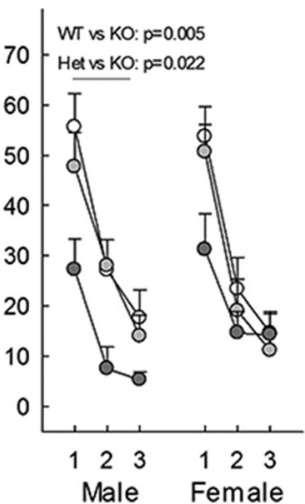

Figure 6. Object avoidance behavior in Shank $3^{\Delta 4-22}$-deficient mice. $\boldsymbol{A}$, Short-term memory measured by the time of interaction with familiar and new object in the novel object recognition test. The test consisted of a training with two identical objects followed $1 \mathrm{~h}$ later by a testing session where one of the object was replaced by a novel object. During the testing session, both wild-type and Shank $3^{\Delta 4-22}$ heterozygous mice had a strong preference for the novel object over the familiar object, while Shank $3^{\Delta 4-22}$ homozygous mice failed to display a preference. However, this failure was due to an avoidance of both objects as shown by the strong decrease in object interaction and the increase in latency to explore any of the object for the first time in Shank $3^{\Delta 4-22}$ homozygous animals, rather than to a real lack of object preference. Representative heatmaps for the three genotypes are available in Extended Data Figure 6-1A. B, Repetitive behavior and object avoidance measured in the marble burying test by the number of marble buried during a $30-$ min session. Shank $3^{\Delta 4-22}$ homozygous mice displayed a strongly impaired burying behavior, leaving most of the marbles undisturbed. Representative pictures and individual data are displayed in Extended Data Figure 6-1B. C, Time spend exploring objects in the repetitive novel object contact task. Shank $3^{\Delta 4-22}$ homozygous mice spent significantly less time interacting with the objects than their wild-type and heterozygous littermates. $\boldsymbol{D}$, Nest building scores. Shank $3^{\Delta 4-22}$ homozygous mice are building less elaborate nests and use less nesting material than their wild-type and heterozygous littermates. Representative pictures of the nests and individual data are displayed in Extended Data Figure 6-1C. $\boldsymbol{E}$, Time interacting with the scent applicator (touching, biting, climbing) during the olfactory habituation/dishabituation test. Shank $3^{\Delta 4-22}$ homozygous mice are avoiding interaction with the scent 
continued

applicator for all nonsocial scents and for a social male scent but have interaction level similar to wild-type and heterozygous animals when presented with a female scent. WT, wild-type mice; Het, heterozygous mice; KO, homozygous knock-out mice. $* p<0.05$, $* * p$ $<0.1, * * * p<0.001$.

consistent with recent results from an independent model also generated by disrupting all Shank3 isoforms (Wang et al., 2016b).

PMS is a neurodevelopmental disorder that manifests as early as in infancy by neonatal hypotonia and a generalized developmental delay. Previous studies have shown normal neonatal development in $\Delta 4-9$ mice (Bozdagi et al., 2010; Wang et al., 2011; Yang et al., 2012) or only minor delays limited to ear opening and paw positioning in $\Delta 4-22$ mice (Wang et al., 2016b). In the current study, both physical and behavioral developmental milestones were investigated. Physical delays were limited to a slower growth rate in Shank $3^{\Delta 4-22}$-deficient animals. In addition, a non-Mendelian genotype distribution showing a deficit for Shank $3^{\Delta 4-22}$ homozygous mice was explained, at least partially, by an increased postnatal mortality observed in the Shank $3^{\Delta 4-22}$ mice homozygous animals. Similar non-Mendelian genotype distributions have been previously observed in other mouse and rat Shank3 models (Drapeau et al., 2014; Harony-Nicolas et al., 2017). As Shank3 is known to be highly expressed in placenta (Beri et al., 2007), this suggests that Shank3 deficiency could lead to placental insufficiency responsible for in utero developmental delays and increased perinatal mortality. Despite a slower growth curves during the first weeks of life, the weight of surviving homozygous animals is no longer different from their littermates when examined at three months of age, indicating a post birth correction, and survival curves between 2 and 22 months do not show any significant genotype difference.

Extensive sensory-motor deficits were observed in newborn Shank3-deficient mice. Some of them, such as the response to an auditory startle or the air righting ability, were only delayed, while others, such as performances in the wire suspension tests and the grasping reflex, were still present at the time of weaning. On homecage observation and physical examination of adult mice we did not observe severe deficits that would preclude advanced testing.

Hypotonia, motor-coordination impairments and gait abnormalities are a hallmark of PMS that persists beyond

Table 10. Detailed results and statistical analyses related to the hyper-reactivity and escape behavior

\begin{tabular}{|c|c|c|c|c|c|c|c|c|c|c|c|c|c|c|c|c|c|}
\hline \multicolumn{18}{|c|}{ Reflexes and reactions to simple stimuli } \\
\hline & \multirow[b]{2}{*}{ Test } & \multirow[b]{2}{*}{$\begin{array}{l}\text { Data } \\
\text { structure }\end{array}$} & \multirow[b]{2}{*}{ WT } & \multirow[b]{2}{*}{ Het } & \multirow[b]{2}{*}{ KO } & \multicolumn{3}{|c|}{ Genotype } & \multicolumn{3}{|l|}{ Cohort } & \multicolumn{3}{|c|}{$\begin{array}{l}\text { Genotype } \times \\
\text { cohort }\end{array}$} & \multicolumn{3}{|c|}{$\begin{array}{l}\text { Pairwise } \\
\text { comparisons }\end{array}$} \\
\hline & & & & & & $F$ & $\begin{array}{l}p \\
\text { value }\end{array}$ & Power & $F$ & $\begin{array}{l}p \\
\text { value }\end{array}$ & Power & $F$ & $\begin{array}{l}p \\
\text { value }\end{array}$ & Power & $\begin{array}{l}\text { WT vs } \\
\text { Het }\end{array}$ & $\begin{array}{l}\text { WT vs } \\
\text { KO }\end{array}$ & $\begin{array}{l}\text { Het vs } \\
\text { KO }\end{array}$ \\
\hline Touch escape & 2WANOVA & Nonnormal & $1.26 \pm 0.1$ & $1.15 \pm 0.11$ & $2 \pm 0.15$ & 12.962 & 0.000 & 0.996 & 0.046 & 0.831 & 0.055 & 0.862 & 0.428 & 0.190 & 0.648 & 0.000 & 0.000 \\
\hline Positional passivity (sum) & 2WANOVA & Nonnormal & $2.15 \pm 0.25$ & $1.84 \pm 0.23$ & $2.84 \pm 0.2$ & 11.737 & 0.000 & 0.992 & 106.722 & 0.000 & 1.000 & 1.993 & 0.147 & 0.393 & 0.034 & 0.011 & 0.000 \\
\hline Positional passivity (score) & 2WANOVA & Nonnormal & $1.78 \pm 0.24$ & $2.21 \pm 0.21$ & $0.94 \pm 0.2$ & 14.029 & 0.000 & 0.998 & 44.935 & 0.000 & 1.000 & 3.871 & 0.027 & 0.675 & 0.034 & 0.004 & 0.000 \\
\hline Catalepsy (4 trials) & 2WANOVA & Nonnormal & $2.98 \pm 0.57$ & $2.75 \pm 0.54$ & $0.56 \pm 0.25$ & 7.578 & 0.001 & 0.933 & 4.681 & 0.035 & 0.565 & 1.116 & 0.336 & 0.236 & 0.836 & 0.001 & 0.002 \\
\hline Trunk curl & 2WANOVA & Nonnormal & $1 \pm 0$ & $0.89 \pm 0.07$ & $1 \pm 0$ & 2.547 & 0.088 & 0.487 & 2.537 & 0.117 & 0.346 & 2.547 & 0.088 & 0.487 & 0.057 & 1.000 & 0.056 \\
\hline $\begin{array}{l}\text { Negative geotaxis, latency } \\
\text { to turn }\end{array}$ & 2WANOVA & Nonnormal & $6.73 \pm 1.06$ & $8.21 \pm 1.9$ & $3.22 \pm 0.58$ & 4.201 & 0.020 & 0.713 & 2.978 & 0.090 & 0.395 & 0.707 & 0.498 & 0.163 & 0.499 & 0.042 & 0.008 \\
\hline \multicolumn{18}{|l|}{ Beam walking } \\
\hline & & & & & & \multicolumn{3}{|c|}{ Genotype } & \multicolumn{3}{|l|}{ Cohort } & \multicolumn{3}{|c|}{$\begin{array}{l}\text { Genotype } \times \\
\text { cohort }\end{array}$} & \multicolumn{3}{|c|}{$\begin{array}{l}\text { Pairwise } \\
\text { comparisons }\end{array}$} \\
\hline & Test & $\begin{array}{l}\text { Data } \\
\text { structure }\end{array}$ & WT & Het & KO & $F$ & $\begin{array}{l}p \\
\text { value }\end{array}$ & Power & $F$ & $\begin{array}{l}p \\
\text { value }\end{array}$ & Power & $F$ & $\begin{array}{l}p \\
\text { value }\end{array}$ & Power & $\begin{array}{l}\text { WT vs } \\
\text { Het }\end{array}$ & $\begin{array}{l}\text { WT vs } \\
\text { KO }\end{array}$ & $\begin{array}{l}\text { Het vs } \\
\text { KO }\end{array}$ \\
\hline $\begin{array}{l}\text { Latency to } \\
\text { cross (large, s) }\end{array}$ & 2WANOVA & Nonnormal & $8.34 \pm 2.79$ & $10.8 \pm 4.52$ & $3.96 \pm 1.75$ & 1.163 & 0.321 & 0.244 & 0.251 & 0.618 & 0.078 & 1.300 & 0.281 & 0.269 & - & - & - \\
\hline Latency to cross (medium, s) & 2WANOVA & Nonnormal & $15.9 \pm 4.8$ & $7.17 \pm 4.16$ & $7.65 \pm 6.13$ & 0.811 & 0.450 & 0.181 & 3.349 & 0.073 & 0.435 & 0.121 & 0.887 & 0.068 & - & - & - \\
\hline Latency to cross (small, s) & 2WANOVA & Nonnormal & $54.71 \pm 5.89$ & $46.15 \pm 7.59$ & $33.72 \pm 7.84$ & 2.204 & 0.121 & 0.430 & 0.235 & 0.630 & 0.076 & 0.437 & 0.648 & 0.117 & - & - & - \\
\hline \multicolumn{18}{|l|}{ Escape behavior } \\
\hline & & & & & & \multicolumn{2}{|c|}{ Genotype } & & \multicolumn{2}{|l|}{ Cohort } & & \multicolumn{2}{|c|}{$\begin{array}{l}\text { Genotype } \times \\
\text { cohort }\end{array}$} & & \multicolumn{2}{|c|}{$\begin{array}{l}\text { Pairwise } \\
\text { comparisons }\end{array}$} & \\
\hline & Test & $\begin{array}{l}\text { Data } \\
\text { structure }\end{array}$ & WT & Het & KO & $F$ & $\begin{array}{l}p \\
\text { value }\end{array}$ & Power & $F$ & $\begin{array}{l}p \\
\text { value }\end{array}$ & Power & $F$ & $\begin{array}{l}p \\
\text { value }\end{array}$ & Power & $\begin{array}{l}\text { WT vs } \\
\text { Het }\end{array}$ & $\begin{array}{l}\text { WT vs } \\
\text { KO }\end{array}$ & $\begin{array}{l}\text { Het vs } \\
\text { KO }\end{array}$ \\
\hline $\begin{array}{l}\text { Buried food, number of } \\
\text { attempts }\end{array}$ & 2WANOVA & Nonnormal & $0.63 \pm 0.35$ & $0.68 \pm 0.32$ & $0.42 \pm 0.31$ & 0.666 & 0.519 & 0.155 & 1.738 & 0.194 & 0.253 & 0.220 & 0.804 & 0.082 & & & \\
\hline $\begin{array}{l}\text { Buried food, \% of } \\
\text { escapers }\end{array}$ & 2WANOVA & Nonnormal & $21.05 \pm 9.6$ & $26.31 \pm 10.37$ & $15.78 \pm 8.59$ & 0.760 & 0.473 & 0.172 & 3.639 & 0.062 & 0.464 & 0.159 & 0.853 & 0.073 & & & \\
\hline $\begin{array}{l}\text { Four-object, number } \\
\text { of attempts }\end{array}$ & 2WANOVA & Nonnormal & $0.41 \pm 0.21$ & $2.05 \pm 0.73$ & $3.88 \pm 1.21$ & 5.323 & 0.008 & 0.815 & 5.320 & 0.025 & 0.618 & 3.316 & 0.045 & 0.601 & 0.187 & 0.002 & 0.050 \\
\hline $\begin{array}{l}\text { Four-object, \% of } \\
\text { escapers }\end{array}$ & 2WANOVA & Nonnormal & $23.52 \pm 10.6$ & $36.84 \pm 11.36$ & $50 \pm 12.12$ & 1.502 & 0.233 & 0.305 & 4.351 & 0.042 & 0.534 & 2.575 & 0.087 & 0.490 & & & \\
\hline $\begin{array}{l}\text { Marble burying, number } \\
\text { of attempts }\end{array}$ & 2WANOVA & Nonnormal & $4.32 \pm 1.28$ & $10.63 \pm 1.98$ & $16.05 \pm 2.38$ & 8.063 & 0.001 & 0.946 & 6.649 & 0.013 & 0.715 & 1.239 & 0.299 & 0.257 & 0.034 & 0.000 & 0.055 \\
\hline $\begin{array}{l}\text { Marble burying, \% of } \\
\text { escapers }\end{array}$ & 2WANOVA & Nonnormal & $47.36 \pm 11.76$ & $94.73 \pm 5.26$ & $100 \pm 0$ & 12.009 & 0.000 & 0.993 & 7.713 & 0.008 & 0.777 & 4.474 & 0.017 & 0.740 & 0.000 & 0.000 & 0.598 \\
\hline
\end{tabular}

WT, wild-type mice; Het, heterozygous mice; KO, homozygous knock-out mice. Group values are reported as mean \pm SEM. Red font indicates significant results $(p<$ 0.05). Individual results and statistical analyses for cohorts 1 and 2 are available in Extended Data Table 10-1. 2wANOVA: 2-way ANOVA. 
A

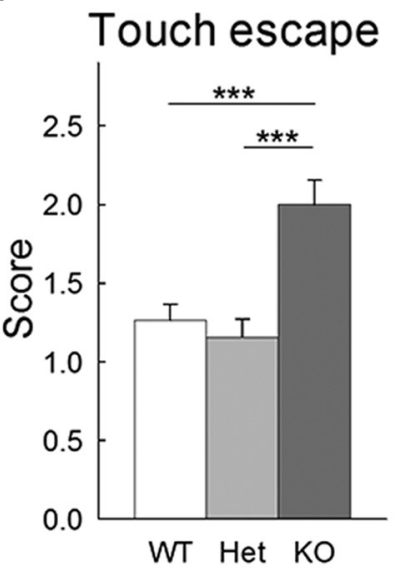

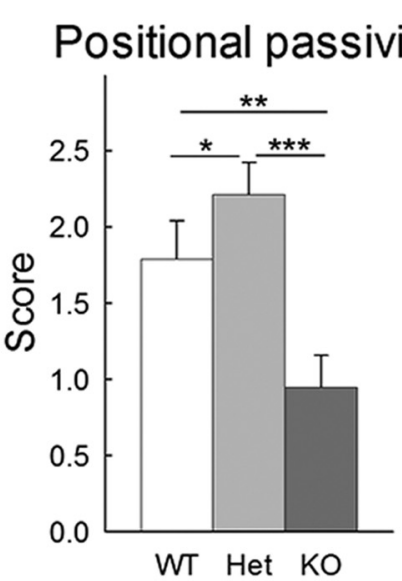

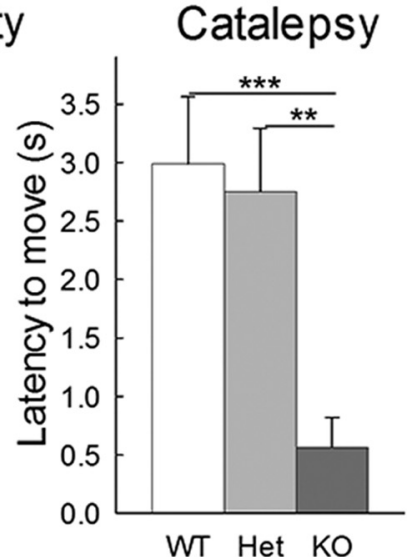

B
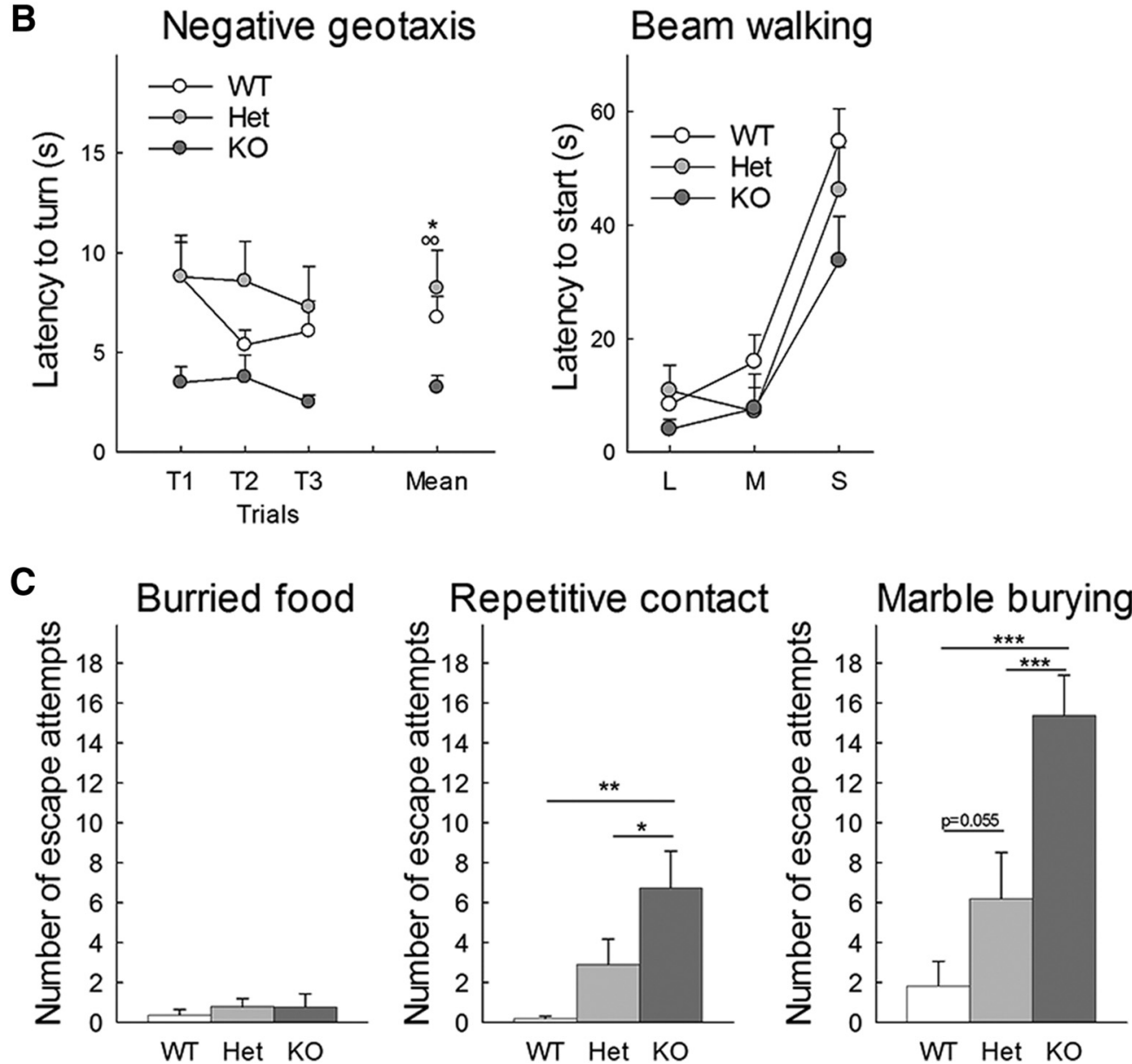

Figure 7. Hyper-reactivity and escape behavior in Shank $3^{\Delta 4-22}$-deficient mice. $\boldsymbol{A}$, Hyper-reactivity measured by animal response in touch escape, positional passivity, and catalepsy. Shank $3^{\Delta 4-22}$ homozygous mice have hyper-reactive responses as shown by a higher score in the touch escape indicating an escape response to lighter strokes, a lower score in positional passivity indicating that they struggle more when restrained, and a lower latency to get off a rdownod in the catalepsy test. $\boldsymbol{B}$, Impulsivity in the negative geotaxis and beam walking tests. The latency to start turning in the negative geotaxis test and to start crossing in the beam walking test are significantly lower in Shank $3^{\Delta 4-22}$ homozygous mice compared to their wild-type and heterozygous littermates and often associated with higher failure rates (data not shown) thus demonstrating impulsive behavior. $\boldsymbol{C}$, Escape behavior measured in different tests with increased inanimate object exposure. No escape attempts were observed for any genotype during the habituation phase of the buried food test (empty home cage with clean bedding). Object exposure induced a significant escape behavior in Shank $3^{\Delta 4-22}$ homozygous mice with a number of attempts increasing with the number of objects in the cage (same home cage, four objects in the repetitive novel object contact task, 20 objects in the marble burying test). Very little escape attempts were observed in wild-type mice, while an intermediate phenotype was observed in heterozygous mice. WT, wild-type mice; Het, heterozygous mice; KO, homozygous knock-out mice. *: WT vs KO; o: WT vs Het. $* p<0.05, * * p<0.1, * * * p<0.001$. 
Table 11. Detailed results and statistical analyses related to stereotypies, repetitive behavior, perseveration, and cognitive flexibility

\begin{tabular}{|c|c|c|c|c|c|c|c|c|c|c|c|c|c|c|c|c|c|}
\hline \multicolumn{18}{|l|}{ Sterotypies in open field } \\
\hline & \multirow[b]{2}{*}{ Test } & \multirow[b]{2}{*}{$\begin{array}{l}\text { Data } \\
\text { structure }\end{array}$} & \multirow[b]{2}{*}{ WT } & \multirow[b]{2}{*}{ Het } & \multirow[b]{2}{*}{$\mathrm{KO}$} & \multicolumn{3}{|c|}{ Genotype } & \multicolumn{3}{|l|}{ Cohort } & $\begin{array}{l}\text { Genoty } \\
\text { cohort }\end{array}$ & pe $\times$ & & $\begin{array}{l}\text { Pairwise } \\
\text { compari }\end{array}$ & $\begin{array}{l}\text { e } \\
\text { isons }\end{array}$ & \\
\hline & & & & & & $F$ & $\begin{array}{l}p \\
\text { value }\end{array}$ & Power & $F$ & $\begin{array}{l}p \\
\text { value }\end{array}$ & Power & $F$ & $\begin{array}{l}p \\
\text { value }\end{array}$ & Power & $\begin{array}{l}\text { WT vs } \\
\text { Het }\end{array}$ & $\begin{array}{l}\text { WT vs } \\
\text { KO }\end{array}$ & $\begin{array}{l}\text { Het vs } \\
\text { KO }\end{array}$ \\
\hline Grooming, time (s) & 2WANOVA & Nonnormal & $67.01 \pm 7.01$ & $62.6 \pm 5.58$ & $92.49 \pm 10.45$ & 4.929 & 0.011 & 0.784 & 22.806 & 0.000 & 0.997 & 2.530 & 0.090 & 0.484 & 0.883 & 0.023 & 0.006 \\
\hline Grooming, number & 2WANOVA & Normal & $25.42 \pm 1.51$ & $22.26 \pm 1.5$ & $27.1 \pm 1.93$ & 2.000 & 0.146 & 0.394 & 1.402 & 0.242 & 0.213 & 1.745 & 0.185 & 0.349 & - & - & - \\
\hline Jumping, time (s) & 2WANOVA & Nonnormal & $0.07 \pm 0.05$ & $0 \pm 0$ & $0.18 \pm 0.1$ & 1.666 & 0.199 & 0.335 & 0.033 & 0.857 & 0.054 & 1.149 & 0.325 & 0.242 & - & - & - \\
\hline Jumping, number & 2WANOVA & Nonnormal & $0.36 \pm 0.23$ & $0 \pm 0$ & $0.42 \pm 0.23$ & 1.300 & 0.281 & 0.269 & 0.155 & 0.696 & 0.067 & 1.816 & 0.173 & 0.362 & - & - & - \\
\hline Rotation, time (s) & 2WANOVA & Nonnormal & $0.39 \pm 0.1$ & $1.49 \pm 0.81$ & $4.21 \pm 2.76$ & 1.560 & 0.220 & 0.316 & 2.069 & 0.156 & 0.292 & 1.038 & 0.361 & 0.222 & - & _- & - \\
\hline Rotation, number & 2WANOVA & Nonnormal & $1.63 \pm 0.39$ & $2.21 \pm 0.46$ & $6.15 \pm 1.82$ & 5.883 & 0.005 & 0.856 & 3.301 & 0.075 & 0.430 & 3.022 & 0.057 & 0.561 & 0.920 & 0.010 & 0.028 \\
\hline Twitching/shaking, time (s) & 2WANOVA & Nonnormal & $0.28 \pm 0.07$ & $0.69 \pm 0.33$ & $0.63 \pm 0.1$ & 1.089 & 0.344 & 0.231 & 0.540 & 0.466 & 0.111 & 0.879 & 0.422 & 0.193 & - & - & - \\
\hline Twitching/shaking, number & 2WANOVA & Nonnormal & $1.73 \pm 0.42$ & $2.63 \pm 0.88$ & $3 \pm 0.47$ & 1.194 & 0.311 & 0.250 & 1.484 & 0.229 & 0.223 & 0.589 & 0.559 & 0.143 & - & - & - \\
\hline Repetitive novel object cc & ontact task, & object prefe & rence, time & & & & & & & & & & & & & & \\
\hline Object exploration, time (s) & Test & $\begin{array}{l}\text { Data } \\
\text { structure }\end{array}$ & & & & $F$ & $\begin{array}{l}p \\
\text { value }\end{array}$ & Power & $\begin{array}{l}\text { WT vs } \\
\text { Het }\end{array}$ & $\begin{array}{l}\text { WT vs } \\
\text { KO }\end{array}$ & $\begin{array}{l}\text { Het vs } \\
\text { KO }\end{array}$ & & & & & & \\
\hline - Object & rMeasures & Sph.viol & & & & 10.533 & 0.000 & 0.999 & - & - & - & & & & & & \\
\hline - Object $\times$ gen. & rMeasures & Sph.viol & & & & 2.150 & 0.069 & 0.753 & - & - & - & & & & & & \\
\hline - Genotype & rMeasures & Sph.viol & & & & 7.964 & 0.001 & 0.943 & 0.956 & 0.014 & 0.006 & & & & & & \\
\hline - Cohort & rMeasures & Sph.viol & & & & 24.654 & 0.000 & 0.998 & - & - & - & & & & & & \\
\hline - Object $\times$ gen. $\times$ coh. & rMeasures & Sph.viol & & & & 0.366 & 0.859 & 0.152 & - & - & - & & & & & & \\
\hline - Gen. × coh. & rMeasures & Sph.viol & & & & 0.647 & 0.528 & 0.152 & - & - & - & & & & & & \\
\hline & & & & & & Genotype & & & Cohort & & & $\begin{array}{l}\text { Genoty } \\
\text { cohort }\end{array}$ & pe $\times$ & & $\begin{array}{l}\text { Pairwise } \\
\text { compar }\end{array}$ & $\begin{array}{l}\text { e } \\
\text { isons }\end{array}$ & \\
\hline Object exploration & Test & $\begin{array}{l}\text { Data } \\
\text { structure }\end{array}$ & WT & Het & KO & $F$ & $\begin{array}{l}p \\
\text { value }\end{array}$ & Power & $F$ & $\begin{array}{l}p \\
\text { value }\end{array}$ & Power & $F$ & $\begin{array}{l}p \\
\text { value }\end{array}$ & Power & $\begin{array}{l}\text { WT vs } \\
\text { Het }\end{array}$ & $\begin{array}{l}\text { WT vs } \\
\text { KO }\end{array}$ & $\begin{array}{l}\text { Het vs } \\
\text { KO }\end{array}$ \\
\hline Dice, time (s) & 2WANOVA & Nonnormal & $16.51 \pm 2.49$ & $14.82 \pm 1.66$ & $12.14 \pm 1.79$ & 1.748 & 0.185 & 0.349 & 4.640 & 0.036 & 0.560 & 0.833 & 0.441 & 0.185 & - & - & - \\
\hline Jack, time (s) & 2WANOVA & Nonnormal & $21.78 \pm 3.18$ & $27.99 \pm 5.1$ & $15.39 \pm 2$ & 4.078 & 0.023 & 0.697 & 14.158 & 0.000 & 0.958 & 0.500 & 0.610 & 0.127 & 0.311 & 0.443 & 0.025 \\
\hline Lego, time (s) & 2WANOVA & Nonnormal & $24.91 \pm 3.59$ & $28.25 \pm 2.94$ & $14.97 \pm 1.87$ & 8.622 & 0.001 & 0.959 & 20.965 & 0.000 & 0.994 & 0.774 & 0.467 & 0.174 & 0.509 & 0.031 & 0.001 \\
\hline Pin, time (s) & 2WANOVA & Nonnormal & $20.8 \pm 4.3$ & $13.72 \pm 1.82$ & $10.86 \pm 1.7$ & 3.199 & 0.050 & 0.585 & 4.532 & 0.038 & 0.550 & 0.057 & 0.944 & 0.058 & 0.244 & 0.067 & 0.755 \\
\hline Object exploration, \% & Test & $\begin{array}{l}\text { Data } \\
\text { structure }\end{array}$ & & & & $F$ & $\begin{array}{l}p \\
\text { value }\end{array}$ & Power & $\begin{array}{l}\text { WT vs } \\
\text { Het }\end{array}$ & $\begin{array}{l}\text { WT vs } \\
\text { KO }\end{array}$ & $\begin{array}{l}\text { Het vs } \\
\text { KO }\end{array}$ & & & & & & \\
\hline - Object & rMeasures & Sph.ass & & & & 8.329 & 0.000 & 0.985 & - & - & - & & & & & & \\
\hline - Object $\times$ gen. & rMeasures & Sph.ass & & & & 0.721 & 0.633 & 0.259 & - & - & - & & & & & & \\
\hline - Genotype & rMeasures & Sph.ass & & & & 0.750 & 0.478 & 0.170 & - & - & - & & & & & & \\
\hline - Cohort & rMeasures & Sph.ass & & & & 0.000 & 1.000 & 0.050 & - & - & - & & & & & & \\
\hline - Object $\times$ gen. $\times$ coh . & rMeasures & Sph.ass & & & & 0.652 & 0.688 & 0.236 & - & - & - & & & & & & \\
\hline - Gen. $\times$ coh. & rMeasures & Sph.ass & & & & 0.000 & 1.000 & 0.050 & - & - & - & & & & & & \\
\hline & & & & & & Genotype & & & Cohort & & & $\begin{array}{l}\text { Genoty } \\
\text { cohort }\end{array}$ & pe $\times$ & & $\begin{array}{l}\text { Pairwise } \\
\text { compar }\end{array}$ & $\begin{array}{l}\text { e } \\
\text { isons }\end{array}$ & \\
\hline Object exploration & Test & $\begin{array}{l}\text { Data } \\
\text { structure }\end{array}$ & WT & Het & KO & $F$ & $\begin{array}{l}p \\
\text { value }\end{array}$ & Power & $F$ & $\begin{array}{l}p \\
\text { value }\end{array}$ & Power & $F$ & $\begin{array}{l}p \\
\text { value }\end{array}$ & Power & $\begin{array}{l}\text { WT vs } \\
\text { Het }\end{array}$ & $\begin{array}{l}\text { WT vs } \\
\text { KO }\end{array}$ & $\begin{array}{l}\text { Het vs } \\
\text { KO }\end{array}$ \\
\hline Dice, time (\%) & 2wANOVA & Nonnormal & $20.09 \pm 1.78$ & $19.13 \pm 2.11$ & $24.11 \pm 3.72$ & 1.259 & 0.293 & 0.261 & 4.635 & 0.036 & 0.560 & 1.179 & 0.316 & 0.246 & - & - & - \\
\hline Jack, time (\%) & 2WANOVA & Nonnormal & $26.86 \pm 3.3$ & $30.29 \pm 3.61$ & $28.38 \pm 3.26$ & 0.221 & 0.803 & 0.083 & 1.529 & 0.222 & 0.228 & 0.022 & 0.978 & 0.053 & - & - & - \\
\hline Lego, time (\%) & 2wANOVA & Normal & $29.85 \pm 2.19$ & $32.98 \pm 2.28$ & $27.98 \pm 2.5$ & 1.174 & 0.318 & 0.245 & 0.986 & 0.326 & 0.164 & 0.072 & 0.931 & 0.060 & - & - & - \\
\hline Pin, time (\%) & 2WANOVA & Nonnormal & $23.18 \pm 3.02$ & $17.57 \pm 2.65$ & $19.51 \pm 2.32$ & 0.701 & 0.501 & 0.161 & 0.186 & 0.669 & 0.071 & 1.684 & 0.196 & 0.337 & - & - & - \\
\hline $\begin{array}{l}\text { Object exploration ranked } \\
\text { by preference }\end{array}$ & Test & $\begin{array}{l}\text { Data } \\
\text { structure }\end{array}$ & & & & $F$ & $\begin{array}{l}p \\
\text { value }\end{array}$ & Power & $\begin{array}{l}\text { WT vs } \\
\text { Het }\end{array}$ & $\begin{array}{l}\text { WT vs } \\
\text { KO }\end{array}$ & $\begin{array}{l}\text { Het vs } \\
\text { KO }\end{array}$ & & & & & & \\
\hline - Object & rMeasures & Sph.viol & & & & 110.887 & 0.000 & 1.000 & - & - & - & & & & & & \\
\hline - Object $\times$ gen. & rMeasures & Sph.viol & & & & 5.483 & 0.002 & 0.996 & - & - & - & & & & & & \\
\hline - Genotype & rMeasures & Sph.viol & & & & 8.054 & 0.001 & 0.946 & 0.948 & 0.014 & 0.006 & & & & & & \\
\hline - Cohort & rMeasures & Sph.viol & & & & 24.578 & 0.000 & 0.998 & - & - & - & & & & & & \\
\hline - Object $\times$ gen. $\times$ coh & rMeasures & Sph.viol & & & & 1.187 & 0.321 & 0.457 & - & - & - & & & & & & \\
\hline - Gen. $\times$ coh. & rMeasures & Sph.viol & & & & 0.643 & 0.530 & 0.152 & - & - & - & & & & & & \\
\hline & & & & & & Genotype & & & Cohort & & & $\begin{array}{l}\text { Genoty } \\
\text { cohort }\end{array}$ & pe $\times$ & & $\begin{array}{l}\text { Pairwise } \\
\text { compar }\end{array}$ & $\begin{array}{l}\text { e } \\
\text { isons }\end{array}$ & \\
\hline Object exploration & Test & $\begin{array}{l}\text { Data } \\
\text { structure }\end{array}$ & WT & Het & KO & $F$ & $\begin{array}{l}p \\
\text { value }\end{array}$ & Power & $F$ & $\begin{array}{l}p \\
\text { value }\end{array}$ & Power & $F$ & $\begin{array}{l}p \\
\text { value }\end{array}$ & Power & $\begin{array}{l}\text { WT vs } \\
\text { Het }\end{array}$ & $\begin{array}{l}\text { WT vs } \\
\text { KO }\end{array}$ & $\begin{array}{l}\text { Het vs } \\
\text { KO }\end{array}$ \\
\hline Object \#1, time (s) & 2WANOVA & Nonnormal & $31.83 \pm 3.59$ & $37.45 \pm 4.09$ & $20.53 \pm 1.64$ & 9.051 & 0.000 & 0.967 & 15.093 & 0.000 & 0.968 & 0.934 & 0.400 & 0.202 & 0.264 & 0.048 & 0.001 \\
\hline Object \#2, time (s) & 2WANOVA & Nonnormal & $24.5 \pm 3.27$ & $22.95 \pm 2.14$ & $15.23 \pm 1.78$ & 6.709 & 0.003 & 0.899 & 22.529 & 0.000 & 0.996 & 0.456 & 0.637 & 0.120 & 0.941 & 0.016 & 0.033 \\
\hline Object \#3, time (s) & 2wANOVA & Nonnormal & $15.68 \pm 2.69$ & $15.29 \pm 1.56$ & $10.48 \pm 1.15$ & 4.224 & 0.020 & 0.714 & 20.037 & 0.000 & 0.992 & 1.306 & 0.280 & 0.269 & 0.998 & 0.094 & 0.102 \\
\hline Object \#4, time (s) & 2WANOVA & Nonnormal & $12 \pm 2.41$ & $9.34 \pm 0.96$ & $7.12 \pm 0.99$ & 3.763 & 0.030 & 0.660 & 12.155 & 0.001 & 0.927 & 0.630 & 0.537 & 0.149 & 0.451 & 0.074 & 0.533 \\
\hline $\begin{array}{l}\text { Object exploration,ranked } \\
\text { by preference }\end{array}$ & Test & $\begin{array}{l}\text { Data } \\
\text { structure }\end{array}$ & & & & $F$ & $\begin{array}{l}p \\
\text { value }\end{array}$ & Power & $\begin{array}{l}\text { WT vs } \\
\text { Het }\end{array}$ & $\begin{array}{l}\text { WT vs } \\
\text { KO }\end{array}$ & $\begin{array}{l}\text { Het vs } \\
\text { KO }\end{array}$ & & & & & & \\
\hline - Object & rMeasures & Sph.viol & & & & 146.534 & 0.000 & 1.000 & - & - & - & & & & & & \\
\hline - Object $\times$ gen. & rMeasures & Sph.viol & & & & 0.832 & 0.490 & 0.321 & - & - & - & & & & & & \\
\hline - Genotype & rMeasures & Sph.viol & & & & 0.812 & 0.450 & 0.181 & - & - & - & & & & & & \\
\hline - Cohort & rMeasures & Sph.viol & & & & 0.783 & 0.381 & 0.140 & - & - & - & & & & & & \\
\hline - Object $\times$ gen. $\times$ coh. & rMeasures & Sph.viol & & & & 1.054 & 0.377 & 0.407 & - & - & - & & & & & & \\
\hline - Gen. $\times$ coh. & rMeasures & Sph.viol & & & & 0.812 & 0.450 & 0.181 & - & - & - & & & & & & \\
\hline & & & & & & Genotype & & & Cohort & & & $\begin{array}{l}\text { Genoty } \\
\text { cohort }\end{array}$ & pe $\times$ & & $\begin{array}{l}\text { Pairwis } \\
\text { compar }\end{array}$ & $\begin{array}{l}\text { e } \\
\text { isons }\end{array}$ & \\
\hline Object exploration & Test & $\begin{array}{l}\text { Data } \\
\text { structure }\end{array}$ & WT & Het & $\mathrm{KO}$ & $F$ & $\begin{array}{l}p \\
\text { value }\end{array}$ & Power & $F$ & $\begin{array}{l}p \\
\text { value }\end{array}$ & Power & $F$ & $\begin{array}{l}p \\
\text { value }\end{array}$ & Power & $\begin{array}{l}\text { WT vs } \\
\text { Het }\end{array}$ & $\begin{array}{l}\text { WT vs } \\
\text { KO }\end{array}$ & $\begin{array}{l}\text { Het vs } \\
\text { KO }\end{array}$ \\
\hline Object \#1, time (\%) & 2WANOVA & Nonnormal & $39.5 \pm 2.19$ & $43 \pm 1.71$ & $40.58 \pm 2.74$ & 1.029 & 0.365 & 0.219 & 1.745 & 0.193 & 0.254 & 0.963 & 0.389 & 0.207 & - & - & - \\
\hline Object \#2, time (\%) & 2WANOVA & Nonnormal & $28.98 \pm 1.16$ & $27.21 \pm 1.2$ & $27.58 \pm 1.39$ & 0.805 & 0.453 & 0.180 & 1.231 & 0.273 & 0.193 & 2.400 & 0.102 & 0.461 & - & - & - \\
\hline Object \#3, time (\%) & 2WANOVA & Normal & $17.99 \pm 1.18$ & $18.73 \pm 1.21$ & $19.28 \pm 1.12$ & 0.093 & 0.911 & 0.063 & 0.228 & 0.635 & 0.076 & 0.347 & 0.709 & 0.102 & - & - & - \\
\hline Object \#4, time (s) & 2WANOVA & Normal & $13.51 \pm 0.92$ & $11.62 \pm 0.99$ & $\begin{array}{r}12.54 \pm 1.16 \\
\text { (Con }\end{array}$ & 1.091 & 0.344 & 0.230 & 0.423 & 0.518 & 0.098 & 0.747 & 0.479 & 0.169 & - & - & - \\
\hline
\end{tabular}


Table 11. Continued

\begin{tabular}{lll}
\hline Repetitive novel object contact task, object preference \\
Object interactions, number & Test $\begin{array}{l}\text { Data } \\
\text { structure }\end{array}$ \\
- Object & rMeasures & Sph.viol \\
- Object $\times$ gen. & rMeasures & Sph.viol \\
- Genotype & rMeasures & Sph.viol \\
- Cohort & rMeasures & Sph.viol \\
- Object $\times$ gen. $\times$ coh. & rMeasures & Sph.viol \\
- Gen. $\times$ coh. & rMeasures Sph.viol
\end{tabular}

\begin{tabular}{|c|c|c|}
\hline Exploration numbers & Test & $\begin{array}{l}\text { Data } \\
\text { structure }\end{array}$ \\
\hline Dice & 2WANOVA & Nonnormal \\
\hline Jack & 2WANOVA & Normal \\
\hline Lego & 2WANOVA & Normal \\
\hline Pin & 2WANOVA & Normal \\
\hline Object interaction \% & Test & $\begin{array}{l}\text { Data } \\
\text { structure }\end{array}$ \\
\hline - Object & rMeasures & Sph.viol \\
\hline - Object $\times$ gen. & rMeasures & Sph.viol \\
\hline - Genotype & rMeasures & Sph.viol \\
\hline - Cohort & rMeasures & Sph.viol \\
\hline - Object $\times$ gen. $\times$ coh. & rMeasures & Sph.viol \\
\hline - Gen. $\times$ coh. & rMeasures & Sph.viol \\
\hline
\end{tabular}

$\begin{array}{llllll}F & p & \text { Power } & \text { WT vs } & \text { WT vs } \\ \text { value } & & \text { Het vs } & \text { KO } & \text { KO } \\ 2.653 & 0.051 & 0.638 & - & - & - \\ 0.858 & 0.528 & 0.331 & - & - & - \\ 2.108 & 0.133 & 0.412 & - & - & - \\ 73.475 & 0.000 & 1.000 & - & - & - \\ 0.459 & 0.837 & 0.184 & - & - & - \\ 1.110 & 0.338 & 0.234 & - & - & -\end{array}$

Genotype Cohort

\begin{tabular}{|c|c|c|}
\hline Genotype & Cohor & $\begin{array}{l}\text { Genotype } \times \\
\text { cohort }\end{array}$ \\
\hline
\end{tabular}

\begin{tabular}{|c|c|c|c|c|c|c|c|c|c|c|c|c|c|c|c|c|c|}
\hline \multirow[b]{2}{*}{ Object interaction number } & \multirow[b]{2}{*}{ Test } & \multirow[b]{2}{*}{$\begin{array}{l}\text { Data } \\
\text { structure }\end{array}$} & \multirow[b]{2}{*}{ WT } & \multirow[b]{2}{*}{ Het } & \multirow[b]{2}{*}{ KO } & \multicolumn{3}{|c|}{ Genotype } & \multicolumn{3}{|l|}{ Cohort } & \multicolumn{3}{|c|}{$\begin{array}{l}\text { Genotype } \times \\
\text { cohort }\end{array}$} & \multicolumn{3}{|c|}{$\begin{array}{l}\text { Pairwise } \\
\text { comparisons }\end{array}$} \\
\hline & & & & & & $F$ & $\begin{array}{l}p \\
\text { value }\end{array}$ & Power & $F$ & $\begin{array}{l}p \\
\text { value }\end{array}$ & Power & $F$ & $\begin{array}{l}p \\
\text { value }\end{array}$ & Power & $\begin{array}{l}\text { WT vs } \\
\text { Het }\end{array}$ & $\begin{array}{l}\text { WT vs } \\
\text { KO }\end{array}$ & $\begin{array}{l}\text { Het vs } \\
\text { KO }\end{array}$ \\
\hline Dice & 2WANOVA & Normal & $24.87 \pm 1.44$ & $23.13 \pm 1.27$ & $25.81 \pm 1.7$ & 1.119 & 0.335 & 0.235 & 10.075 & 0.003 & 0.875 & 0.297 & 0.745 & 0.094 & - & - & - \\
\hline Jack & 2WANOVA & Normal & $23.14 \pm 1.35$ & $24.09 \pm 1.09$ & $24.69 \pm 0.91$ & 0.164 & 0.849 & 0.074 & 11.956 & 0.001 & 0.923 & 0.546 & 0.583 & 0.135 & - & - & - \\
\hline Lego & 2WANOVA & Nonnormal & $28.84 \pm 2$ & $28.66 \pm 1.57$ & $24.78 \pm 1.41$ & 1.507 & 0.232 & 0.305 & 2.901 & 0.095 & 0.386 & 1.286 & 0.286 & 0.265 & - & - & - \\
\hline Pin & 2WANOVA & Nonnormal & $33.55 \pm 7.69$ & $32.7 \pm 6.64$ & $30.85 \pm 4.83$ & 0.235 & 0.792 & 0.085 & 10.476 & 0.002 & 0.887 & 0.467 & 0.630 & 0.122 & - & - & - \\
\hline $\begin{array}{l}\text { Object interaction number, } \\
\text { object ranked by preference }\end{array}$ & Test & $\begin{array}{l}\text { Data } \\
\text { structure }\end{array}$ & & & & $F$ & $\begin{array}{l}p \\
\text { value }\end{array}$ & Power & $\begin{array}{l}\text { WT vs } \\
\text { Het }\end{array}$ & $\begin{array}{l}\text { WT vs } \\
\text { KO }\end{array}$ & $\begin{array}{l}\text { Het vs } \\
\text { KO }\end{array}$ & & & & & & \\
\hline - Object & rMeasures & Sph.viol & & & & 74.224 & 0.000 & 1.000 & - & - & - & & & & & & \\
\hline - Object $\times$ gen. & rMeasures & Sph.viol & & & & 0.867 & 0.499 & 0.335 & - & - & - & & & & & & \\
\hline - Genotype & rMeasures & Sph.viol & & & & 2.228 & 0.119 & 0.432 & - & - & - & & & & & & \\
\hline - Cohort & rMeasures & Sph.viol & & & & 72.229 & 0.000 & 1.000 & - & - & - & & & & & & \\
\hline - Object $\times$ gen. $\times$ coh. & rMeasures & Sph.viol & & & & 0.653 & 0.649 & 0.254 & - & - & - & & & & & & \\
\hline \multirow[t]{2}{*}{ - Gen. $\times$ coh. } & rMeasures & Sph.viol & & & & 1.142 & 0.328 & 0.239 & - & - & - & & & & & & \\
\hline & & & & & & \multicolumn{3}{|c|}{ Genotype } & Cohort & & & \multicolumn{3}{|c|}{$\begin{array}{l}\text { Genotype } \times \\
\text { cohort }\end{array}$} & \multicolumn{3}{|c|}{$\begin{array}{l}\text { Pairwise } \\
\text { comparisons }\end{array}$} \\
\hline
\end{tabular}

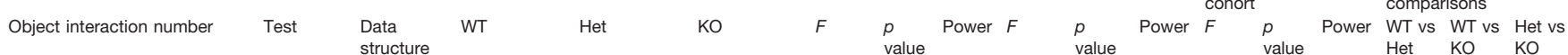

$\begin{array}{lll}\text { Object \#1 } & \text { 2wANOVA } & \text { Normal } \\ \text { Object \#2 } & \text { 2wANOVA } & \text { Nonnorm } \\ \text { Object \#3 } & \text { 2wANOVA Nonnorm } \\ \text { Object } \# 4 & \text { 2wANOVA } & \text { Nonnorm } \\ & & \\ \text { Object interaction \%, object } & \text { Test } & \begin{array}{l}\text { Data } \\ \text { structure }\end{array} \\ \text { ranked by preference } & & \text { rMeasures } \\ \text { - Object } & \text { rMeasures } & \text { Sph.viol } \\ \text { - Object } \times \text { gen. } & \text { rMeasures Sph.viol } \\ \text { - Genotype } & \text { rMeasures Sph.viol } \\ \text { - Cohort } & \text { rMeasures Sph.viol } \\ \text { - Object } \times \text { gen. } \times \text { coh. } & \text { rMeasures Sph.viol } \\ \text { - Gen. } \times \text { coh. } & \end{array}$

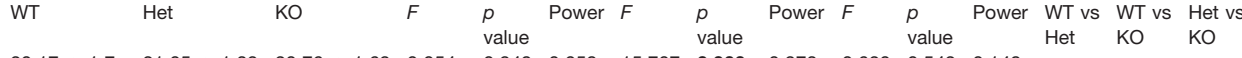

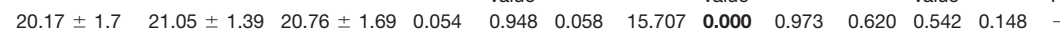

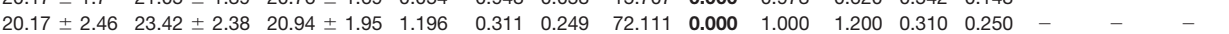

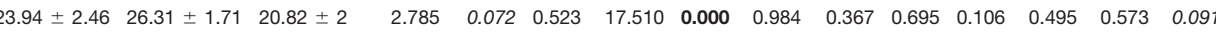

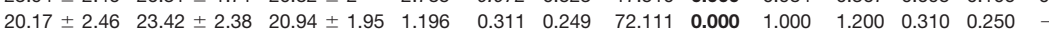

$\begin{array}{llllll}F & p & \text { Power } & \text { WT vs } & \text { WT vs } & \text { Het vs } \\ \text { value } & & \text { Het } & \text { KO } & \text { KO } \\ 4.812 & 0.022 & 0.897 & - & - & - \\ 0.363 & 0.762 & 0.151 & - & - & - \\ 0.328 & 0.722 & 0.099 & - & - & - \\ 7.375 & 0.009 & 0.758 & - & - & - \\ 0.560 & 0.627 & 0.220 & - & - & - \\ 0.328 & 0.722 & 0.099 & - & - & -\end{array}$


Table 11. Continued

\begin{tabular}{|c|c|c|c|c|c|c|c|c|c|c|c|c|c|c|c|c|c|}
\hline $\begin{array}{l}\text { Number of repetition of top } \\
\text { preferred sequence }\end{array}$ & 2wANOVA & Nonnormal & $4.88 \pm 0.4$ & $4.78 \pm 0.37$ & $4.64 \pm 0.29$ & 0.012 & 0.988 & 0.052 & 10.796 & 0.002 & 0.896 & 0.052 & 0.950 & 0.057 & - & - & - \\
\hline $\begin{array}{l}\text { Number of repetition of } 2 \text { nd } \\
\text { preferred sequence }\end{array}$ & 2wANOVA & Nonnormal & $4.27 \pm 0.27$ & $4.15 \pm 0.33$ & $4.05 \pm 0.26$ & 0.017 & 0.983 & 0.052 & 8.748 & 0.005 & 0.826 & 0.312 & 0.733 & 0.097 & - & - & - \\
\hline $\begin{array}{l}\text { Number of repetition of } 3 r d \\
\text { preferred sequence }\end{array}$ & 2wANOVA & Nonnormal & $3.83 \pm 0.23$ & $3.68 \pm 0.3$ & $3.7 \pm 0.25$ & 0.042 & 0.959 & 0.056 & 6.542 & 0.014 & 0.708 & 0.303 & 0.740 & 0.095 & - & - & - \\
\hline $\begin{array}{l}\text { Number of repetition of top } \\
3 \text { preferred sequences }\end{array}$ & 2wANOVA & Normal & $13 \pm 0.87$ & $12.63 \pm 0.98$ & $12.41 \pm 0.78$ & 0.008 & 0.992 & 0.051 & 9.545 & 0.003 & 0.857 & 0.146 & 0.865 & 0.071 & - & - & - \\
\hline $\begin{array}{l}\% \text { top preferred sequence } \\
\text { choice }\end{array}$ & 2wANOVA & Nonnormal & $8.57 \pm 0.32$ & $9.41 \pm 0.61$ & $8.75 \pm 0.19$ & 1.324 & 0.276 & 0.272 & 0.000 & 0.994 & 0.050 & 1.436 & 0.248 & 0.293 & - & - & - \\
\hline $\begin{array}{l}\% \text { top } 2 \text { preferred sequence } \\
\text { choice }\end{array}$ & 2WANOVA & Nonnormal & $16.22 \pm 0.52$ & $17.48 \pm 1.01$ & $16.43 \pm 0.32$ & 1.179 & 0.316 & 0.246 & 0.143 & 0.707 & 0.066 & 1.543 & 0.224 & 0.312 & - & - & - \\
\hline \multirow[t]{2}{*}{$\begin{array}{l}\% \text { top } 3 \text { preferred sequence } \\
\text { choice }\end{array}$} & 2wANOVA & Nonnormal & $23.14 \pm 0.71$ & $24.69 \pm 1.43$ & $23.44 \pm 0.51$ & 0.837 & 0.439 & 0.185 & 0.564 & 0.456 & 0.114 & 1.040 & 0.361 & 0.221 & - & - & - \\
\hline & & & & & & \multicolumn{2}{|l|}{ Genotype } & & \multicolumn{2}{|l|}{ Cohort } & & \multicolumn{2}{|c|}{$\begin{array}{l}\text { Genotype } \times \\
\text { cohort }\end{array}$} & & \multicolumn{2}{|c|}{$\begin{array}{l}\text { Pairwise } \\
\text { comparisons }\end{array}$} & \\
\hline Four-object sequences & Test & $\begin{array}{l}\text { Data } \\
\text { structure }\end{array}$ & WT & Het & KO & $F$ & $\begin{array}{l}p \\
\text { value }\end{array}$ & Power & $F$ & $\begin{array}{l}p \\
\text { value }\end{array}$ & Power & $F$ & $\begin{array}{l}p \\
\text { value }\end{array}$ & Power & $\begin{array}{l}\text { WT vs } \\
\text { Het }\end{array}$ & $\begin{array}{l}\text { WT vs } \\
\text { KO }\end{array}$ & $\begin{array}{l}\text { Het vs } \\
\text { KO }\end{array}$ \\
\hline $\begin{array}{l}\text { Total number of 4-object } \\
\text { choices }\end{array}$ & 2wANOVA & Normal & $55.55 \pm 3.26$ & $53.26 \pm 4.79$ & $52.58 \pm 3.24$ & 0.067 & 0.935 & 0.060 & 10.400 & 0.002 & 0.885 & 0.528 & 0.593 & 0.132 & - & - & - \\
\hline $\begin{array}{l}\text { Number of different 4-object } \\
\text { sequences }\end{array}$ & 2wANOVA & Normal & $40.77 \pm 1.59$ & $39.63 \pm 2.78$ & $39 \pm 2.03$ & 0.097 & 0.908 & 0.064 & 9.857 & 0.003 & 0.868 & 0.895 & 0.415 & 0.195 & - & - & - \\
\hline $\begin{array}{l}\text { Number of repetition of top } \\
\text { preferred sequence }\end{array}$ & 2WANOVA & Nonnormal & $3.05 \pm 0.2$ & $3.1 \pm 0.2$ & $3.41 \pm 0.17$ & 1.297 & 0.283 & 0.267 & 4.144 & 0.047 & 0.514 & 0.050 & 0.951 & 0.057 & - & - & - \\
\hline $\begin{array}{l}\text { Number of repetition of 2nd } \\
\text { preferred sequence }\end{array}$ & 2WANOVA & Nonnormal & $2.83 \pm 0.2$ & $2.84 \pm 0.2$ & $2.76 \pm 0.18$ & 0.034 & 0.967 & 0.055 & 4.324 & 0.043 & 0.531 & 1.214 & 0.306 & 0.253 & - & - & - \\
\hline $\begin{array}{l}\text { Number of repetition of } 3 r d \\
\text { preferred sequence }\end{array}$ & 2wANOVA & Nonnormal & $2.44 \pm 0.16$ & $2.47 \pm 0.19$ & $2.23 \pm 0.13$ & 0.468 & 0.629 & 0.122 & 4.499 & 0.039 & 0.547 & 1.063 & 0.353 & 0.225 & - & - & - \\
\hline $\begin{array}{l}\text { Number of repetition of top } \\
3 \text { preferred sequences }\end{array}$ & 2WANOVA & Nonnormal & $8.33 \pm 0.53$ & $8.42 \pm 0.55$ & $8.41 \pm 0.42$ & 0.087 & 0.916 & 0.063 & 5.267 & 0.026 & 0.614 & 0.634 & 0.535 & 0.150 & - & - & - \\
\hline $\begin{array}{l}\% \text { top preferred sequence } \\
\text { choice }\end{array}$ & 2wANOVA & Nonnormal & $5.58 \pm 0.3$ & $6.31 \pm 0.47$ & $6.66 \pm 0.27$ & 2.187 & 0.123 & 0.425 & 2.759 & 0.103 & 0.370 & 1.056 & 0.356 & 0.224 & - & - & - \\
\hline $\begin{array}{l}\% \text { top } 2 \text { preferred sequence } \\
\text { choice }\end{array}$ & 2WANOVA & Nonnormal & $10.7 \pm 0.44$ & $11.99 \pm 0.77$ & $12.09 \pm 0.51$ & 1.734 & 0.187 & 0.346 & 2.734 & 0.105 & 0.367 & 1.166 & 0.320 & 0.244 & - & - & - \\
\hline $\begin{array}{l}\% \text { top } 3 \text { preferred sequence } \\
\text { choice }\end{array}$ & 2WANOVA & Nonnormal & $15.14 \pm 0.54$ & $16.93 \pm 1.02$ & $16.43 \pm 0.67$ & 1.557 & 0.221 & 0.314 & 3.181 & 0.081 & 0.416 & 1.237 & 0.299 & 0.257 & - & - & - \\
\hline \multicolumn{18}{|c|}{ Barnes maze initial training - distance } \\
\hline Distance & Test & $\begin{array}{l}\text { Data } \\
\text { structure }\end{array}$ & & & & $F$ & $\begin{array}{l}p \\
\text { value }\end{array}$ & Power & $\begin{array}{l}\text { WT vs } \\
\text { Het }\end{array}$ & $\begin{array}{l}\text { WT vs } \\
\text { KO }\end{array}$ & $\begin{array}{l}\text { Het vs } \\
\mathrm{KO}\end{array}$ & & & & & & \\
\hline - Day & rMeasures & Sph.ass & & & & 13.695 & 0.000 & 1.000 & - & - & - & & & & & & \\
\hline - Day $\times$ gen. & rMeasures & Sph.ass & & & & 2.062 & 0.062 & 0.684 & - & - & - & & & & & & \\
\hline - Genotype & rMeasures & Sph.ass & & & & 2.663 & 0.080 & 0.503 & 0.659 & 0.145 & 0.515 & & & & & & \\
\hline - Cohort & rMeasures & Sph.ass & & & & 11.841 & 0.001 & 0.920 & - & - & - & & & & & & \\
\hline - Day $\times$ gen. $\times$ coh . & rMeasures & Sph.ass & & & & 1.173 & 0.324 & 0.416 & - & - & - & & & & & & \\
\hline \multirow[t]{2}{*}{ - Gen. $\times$ coh. } & rMeasures & Sph.ass & & & & 1.114 & 0.337 & 0.234 & - & - & - & & & & & & \\
\hline & & & & & & \multicolumn{2}{|l|}{ Genotype } & & \multicolumn{2}{|l|}{ Cohort } & & \multicolumn{2}{|c|}{$\begin{array}{l}\text { Genotype } \times \\
\text { cohort }\end{array}$} & & \multicolumn{2}{|c|}{$\begin{array}{l}\text { Pairwise } \\
\text { comparisons }\end{array}$} & \\
\hline Individual days & Test & $\begin{array}{l}\text { Data } \\
\text { structure }\end{array}$ & WT & Het & KO & $F$ & $\begin{array}{l}p \\
\text { value }\end{array}$ & Power & $F$ & $\begin{array}{l}p \\
\text { value }\end{array}$ & Power & $F$ & $\begin{array}{l}p \\
\text { value }\end{array}$ & Power & $\begin{array}{l}\text { WT vs } \\
\text { Het }\end{array}$ & $\begin{array}{l}\text { WT vs } \\
\text { KO }\end{array}$ & $\begin{array}{l}\text { Het vs } \\
\text { KO }\end{array}$ \\
\hline Day 1 & 2wANOVA & Normal & $501.24 \pm 48.28$ & $485.42 \pm 47.71$ & $484.49 \pm 53.07$ & 0.003 & 0.997 & 0.050 & 4.283 & 0.044 & 0.526 & 0.084 & 0.919 & 0.062 & - & - & - \\
\hline Day 2 & 2WANOVA & Normal & $427.6 \pm 43.5$ & $468.59 \pm 40.26$ & $504.18 \pm 47.17$ & 1.234 & 0.301 & 0.256 & 9.205 & 0.004 & 0.844 & 1.918 & 0.158 & 0.378 & - & - & - \\
\hline Day 3 & 2WANOVA & Normal & $292.36 \pm 29.11$ & $340.26 \pm 31.24$ & $485.74 \pm 41.16$ & 11.293 & 0.000 & 0.989 & 6.902 & 0.012 & 0.730 & 3.082 & 0.055 & 0.567 & 0.496 & 0.000 & 0.005 \\
\hline Day 4 & 2WANOVA & Normal & $311.01 \pm 34.75$ & $370.86 \pm 29.61$ & $367.31 \pm 42.11$ & 1.479 & 0.239 & 0.300 & 5.449 & 0.024 & 0.628 & 0.666 & 0.519 & 0.155 & - & - & - \\
\hline \multicolumn{18}{|c|}{ Barnes maze reversal - distance } \\
\hline Distance & Test & $\begin{array}{l}\text { Data } \\
\text { structure }\end{array}$ & & & & $F$ & $\begin{array}{l}p \\
\text { value }\end{array}$ & Power & $\begin{array}{l}\text { WT vs } \\
\text { Het }\end{array}$ & $\begin{array}{l}\text { WT vs } \\
\text { KO }\end{array}$ & $\begin{array}{l}\text { Het vs } \\
\text { KO }\end{array}$ & & & & & & \\
\hline - Day effect & rMeasures & Sph.ass & & & & 26.455 & 0.000 & 1.000 & - & - & - & & & & & & \\
\hline - Day × gen. & rMeasures & Sph.ass & & & & 2.612 & 0.023 & 0.824 & - & - & - & & & & & & \\
\hline - Genotype & rMeasures & Sph.ass & & & & 1.811 & 0.175 & 0.359 & - & - & - & & & & & & \\
\hline - Cohort & rMeasures & Sph.ass & & & & 1.924 & 0.172 & 0.274 & - & - & - & & & & & & \\
\hline - Day $\times$ gen. $\times$ coh . & rMeasures & Sph.ass & & & & 3.192 & 0.007 & 0.902 & - & - & - & & & & & & \\
\hline \multirow[t]{2}{*}{ - Genotype $\times$ cohort effect } & rMeasures & Sph.ass & & & & 0.290 & 0.750 & 0.093 & - & - & - & & & & & & \\
\hline & & & & & & \multicolumn{2}{|c|}{ Genotype } & & Cohort & & & $\begin{array}{l}\text { Genoty } \\
\text { cohort }\end{array}$ & ype $\times$ & & $\begin{array}{l}\text { Pairwis } \\
\text { compar }\end{array}$ & risons & \\
\hline Individual days & Test & $\begin{array}{l}\text { Data } \\
\text { structure }\end{array}$ & WT & Het & $\mathrm{KO}$ & $F$ & $\begin{array}{l}p \\
\text { value }\end{array}$ & Power & $F$ & $\begin{array}{l}p \\
\text { value }\end{array}$ & Power & $F$ & $\begin{array}{l}p \\
\text { value }\end{array}$ & Power & $\begin{array}{l}\text { WT vs } \\
\text { Het }\end{array}$ & $\begin{array}{l}\text { WT vs } \\
\text { KO }\end{array}$ & $\begin{array}{l}\text { Het vs } \\
\text { KO }\end{array}$ \\
\hline Day 1 & 2WANOVA & Nonnormal & $420.93 \pm 37.75$ & $437.03 \pm 37.86$ & $591.59 \pm 38.48$ & 5.592 & 0.007 & 0.834 & 0.793 & 0.378 & 0.141 & 1.475 & 0.239 & 0.299 & 0.948 & 0.009 & 0.018 \\
\hline Day 2 & 2WANOVA & Normal & $336.81 \pm 35.36$ & $413.64 \pm 32.4$ & $390.91 \pm 45$ & 1.285 & 0.286 & 0.265 & 0.374 & 0.544 & 0.092 & 2.525 & 0.091 & 0.481 & - & - & - \\
\hline Day 3 & 2WANOVA & Normal & $357.93 \pm 35.96$ & $421.04 \pm 44.36$ & $395.85 \pm 48.06$ & 0.666 & 0.519 & 0.155 & 3.371 & 0.073 & 0.436 & 0.116 & 0.890 & 0.067 & - & - & - \\
\hline Day 4 & 2WANOVA & Normal & $288.54 \pm 39.85$ & $288.65 \pm 37.41$ & $337.24 \pm 38.59$ & 0.965 & 0.389 & 0.207 & 8.849 & 0.005 & 0.829 & 1.373 & 0.264 & 0.281 & - & - & - \\
\hline Barnes maze initial trainir & probe test & & & & & & & & & & & & & & & & \\
\hline & & & & & & Genotype & & & Quadr & . & ise & parisons & & & & & \\
\hline All animals & Test & $\begin{array}{l}\text { Data } \\
\text { structure }\end{array}$ & & & & $F$ & $\begin{array}{l}p \\
\text { value }\end{array}$ & Power & $\begin{array}{l}\mathrm{T} \text { vs } \\
\mathrm{L}\end{array}$ & $\begin{array}{l}\text { T vs } \\
\mathrm{R}\end{array}$ & $\begin{array}{l}\text { T vs } \\
0\end{array}$ & $\begin{array}{l}\mathrm{L} \text { vs } \\
\mathrm{R}\end{array}$ & $\begin{array}{l}\text { L vs } \\
\text { O }\end{array}$ & $\begin{array}{l}\text { R vs } \\
\mathrm{O}\end{array}$ & & & \\
\hline - Quadrant & rMeasures & Sph.viol & & & & 296.653 & 0.000 & 1.000 & 0.000 & 0.000 & 0.000 & 0.555 & 0.201 & 0.628 & & & \\
\hline - Cohort & rMeasures & Sph.viol & & & & 10.200 & 0.002 & 1.000 & & & & & & & & & \\
\hline - Quadrant $\times$ coh. & rMeasures & Sph.viol & & & & 11.435 & 0.000 & 0.983 & & & & & & & & & \\
\hline & & & & & (Continued) & & & & & & & & & & & & \\
\hline
\end{tabular}


Table 11. Continued

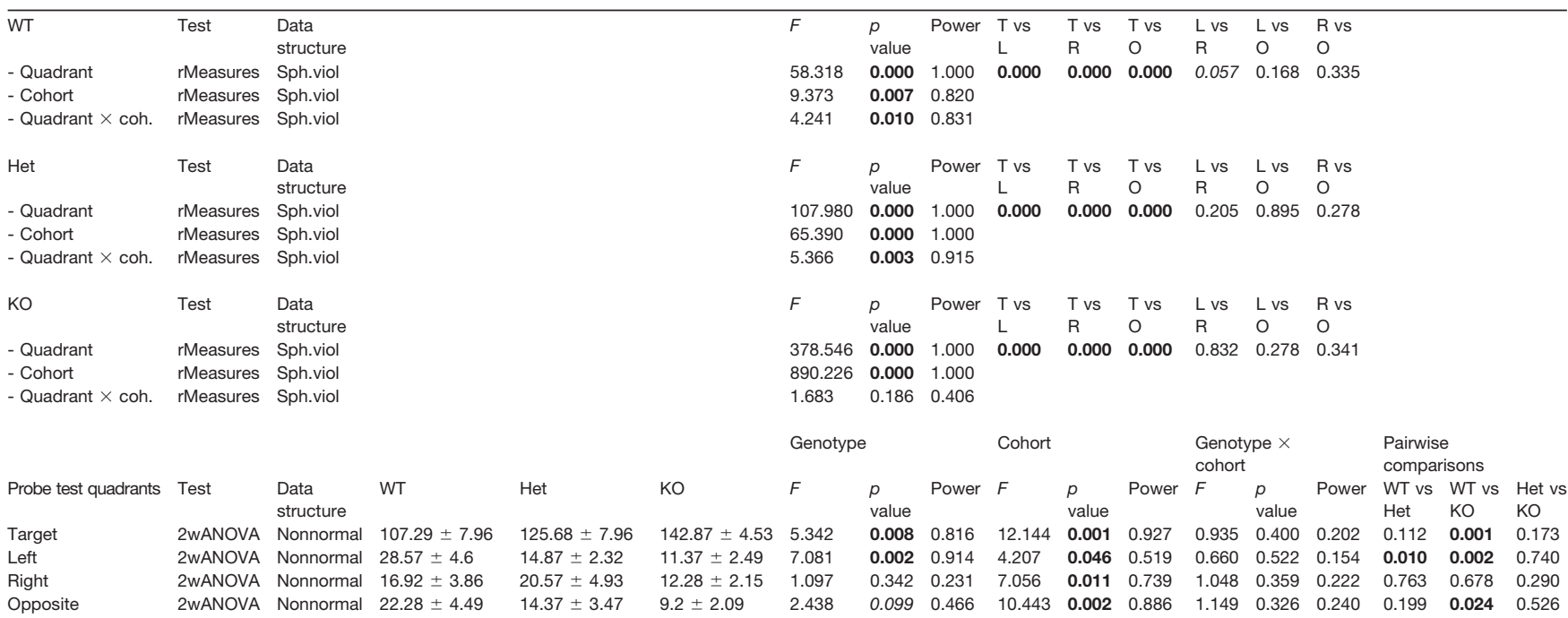

Barnes maze reversal probe test

\begin{tabular}{|c|c|c|}
\hline All animals & Test & $\begin{array}{l}\text { Data } \\
\text { structure }\end{array}$ \\
\hline - Quadrant & rMeasures & Sph.viol \\
\hline - Cohort & rMeasures & Sph.viol \\
\hline - Quadrant $\times$ coh. & rMeasures & Sph.viol \\
\hline WT & Test & $\begin{array}{l}\text { Data } \\
\text { structure }\end{array}$ \\
\hline - Quadrant & rMeasures & Sph.viol \\
\hline - Cohort & rMeasures & Sph.viol \\
\hline - Quadrant $\times$ coh. & rMeasures & Sph.viol \\
\hline Het & Test & $\begin{array}{l}\text { Data } \\
\text { structure }\end{array}$ \\
\hline - Quadrant & rMeasures & Sph.viol \\
\hline - Cohort & rMeasures & Sph.viol \\
\hline - Quadrant $\times$ coh. & rMeasures & Sph.viol \\
\hline $\mathrm{KO}$ & Test & $\begin{array}{l}\text { Data } \\
\text { structure }\end{array}$ \\
\hline - Quadrant & rMeasures & Sph.viol \\
\hline - Cohort & rMeasures & Sph.viol \\
\hline$-\mathrm{Q}$ & rMeasures & Sph.viol \\
\hline
\end{tabular}

\begin{tabular}{|c|c|c|c|c|c|c|c|c|c|c|c|}
\hline \multicolumn{3}{|l|}{ Genotype } & \multicolumn{9}{|c|}{ Quadrant pairwise comparisons } \\
\hline$F$ & $\begin{array}{l}p \\
\text { value }\end{array}$ & Power & $\begin{array}{l}\text { T vs } \\
\mathrm{L}\end{array}$ & $\begin{array}{l}\mathrm{T} \text { vs } \\
\mathrm{R}\end{array}$ & $\begin{array}{l}\text { T vs } \\
0\end{array}$ & $\begin{array}{l}\text { L vs } \\
R\end{array}$ & $\begin{array}{l}\text { L vs } \\
0\end{array}$ & $\begin{array}{l}R \text { vs } \\
O\end{array}$ & & & \\
\hline 50.865 & 0.000 & 1.000 & 0.000 & 0.000 & 0.000 & 0.242 & 0.000 & 0.000 & & & \\
\hline 24.530 & 0.000 & 0.998 & & & & & & & & & \\
\hline 4.443 & 0.005 & 0.870 & & & & & & & & & \\
\hline$F$ & $\begin{array}{l}p \\
\text { value }\end{array}$ & Power & $\begin{array}{l}\text { T vs } \\
\mathrm{L}\end{array}$ & $\begin{array}{l}\mathrm{T} \text { vs } \\
\mathrm{R}\end{array}$ & $\begin{array}{l}\text { T vs } \\
0\end{array}$ & $\begin{array}{l}\mathrm{L} \text { vs } \\
\mathrm{R}\end{array}$ & $\begin{array}{l}\mathrm{L} \text { vs } \\
\mathrm{O}\end{array}$ & $\begin{array}{l}R \text { vs } \\
O\end{array}$ & & & \\
\hline 32.279 & 0.000 & 1.000 & 0.000 & 0.000 & 0.000 & 0.005 & 0.024 & 0.003 & & & \\
\hline 159.377 & 0.000 & 1.000 & & & & & & & & & \\
\hline 0.007 & 0.956 & 0.051 & & & & & & & & & \\
\hline$F$ & $\begin{array}{l}p \\
\text { value }\end{array}$ & Power & $\begin{array}{l}\text { T vs } \\
\mathrm{L}\end{array}$ & $\begin{array}{l}T \text { vs } \\
R\end{array}$ & $\begin{array}{l}\text { T vs } \\
0\end{array}$ & $\begin{array}{l}\text { L vs } \\
R\end{array}$ & $\begin{array}{l}\mathrm{L} \text { vs } \\
\mathrm{O}\end{array}$ & $\begin{array}{l}R \text { vs } \\
O\end{array}$ & & & \\
\hline 28.198 & 0.000 & 1.000 & 0.000 & 0.000 & 0.001 & 0.235 & 0.001 & 0.086 & & & \\
\hline 6.412 & 0.021 & 0.666 & & & & & & & & & \\
\hline 10.315 & 0.000 & 0.998 & & & & & & & & & \\
\hline$F$ & $\begin{array}{l}p \\
\text { value }\end{array}$ & Power & $\begin{array}{l}\text { T vs } \\
\mathrm{L}\end{array}$ & $\begin{array}{l}T \text { vs } \\
R\end{array}$ & $\begin{array}{l}\text { T vs } \\
0\end{array}$ & $\begin{array}{l}\text { L vs } \\
R\end{array}$ & $\begin{array}{l}\mathrm{L} \text { vs } \\
\mathrm{O}\end{array}$ & $\begin{array}{l}R \text { vs } \\
O\end{array}$ & & & \\
\hline 12.026 & 0.000 & 0.999 & 0.000 & 0.010 & 0.646 & 0.070 & 0.000 & 0.000 & & & \\
\hline 397.250 & 0.000 & 1.000 & & & & & & & & & \\
\hline 2.273 & 0.095 & 0.531 & & & & & & & & & \\
\hline Genotype & & & Cohort & & & $\begin{array}{l}\text { Genoty } \\
\text { cohort }\end{array}$ & pe $\times$ & & $\begin{array}{l}\text { Pairwis } \\
\text { compar }\end{array}$ & $\begin{array}{l}\text { re } \\
\text { risons }\end{array}$ & \\
\hline$F$ & $\begin{array}{l}p \\
\text { value }\end{array}$ & Power & $F$ & $\begin{array}{l}p \\
\text { value }\end{array}$ & Power & $F$ & $\begin{array}{l}p \\
\text { value }\end{array}$ & Power & $\begin{array}{l}\text { WT vs } \\
\text { Het }\end{array}$ & $\begin{array}{l}\text { WT vs } \\
\text { KO }\end{array}$ & $\begin{array}{l}\text { Het vs } \\
\mathrm{KO}\end{array}$ \\
\hline 5.430 & 0.008 & 0.822 & 8.183 & 0.006 & 0.800 & 3.469 & 0.040 & 0.621 & 0.773 & 0.010 & 0.046 \\
\hline 3.343 & 0.044 & 0.604 & 2.079 & 0.156 & 0.292 & 0.172 & 0.842 & 0.075 & 0.039 & 0.123 & 0.923 \\
\hline 1.367 & 0.265 & 0.280 & 1.873 & 0.178 & 0.268 & 2.980 & 0.061 & 0.551 & 0.489 & 0.229 & 0.826 \\
\hline 6.632 & 0.003 & 0.894 & 7.210 & 0.010 & 0.748 & 2.097 & 0.134 & 0.409 & 0.662 & 0.004 & 0.030 \\
\hline
\end{tabular}

WT, wild-type mice; Het, heterozygous mice; KO, homozygous knock-out mice. Group values are reported as mean \pm SEM. Bold font indicates significant results $(p<0.05)$. Individual results and statistical analyses for cohorts 1 and 2 are available in Extended Data Table 11-1. 2wANOVA: 2-way ANOVA, rMeasures: repeated measures, Norm: normal, No-norm: non-normal, Sph.ass: sphericity assumed, Sph.viol: sphericity violated, gen: genotype, coh: cohort. 2WANOVA: 2-way ANOVA, rMeasures: repeated measures, Sph.ass: sphericity assumed, Sph.viol: sphericity violated, gen: genotype, coh: cohort.

childhood (Phelan and McDermid, 2012; Soorya et al., 2013). In previous studies, motor performances have been frequently found to be impaired in adult Shank3deficient mice (Fig. 11). Hence, decreased locomotion in the open field has been reported in many existing models including models with $\Delta 4-9, \Delta 13-16, \Delta 21$ deletions, or point mutations (Yang et al., 2012; Kouser et al., 2013; Speed et al., 2015; Bidinosti et al., 2016; Mei et al., 2016; Zhou et al., 2016; Copping et al., 2017) even if not always replicated in other models with similar or different deletions $(\Delta 4-9, \Delta 9, \Delta 13, \Delta 13-16, \Delta 21$; Peça et al., 2011; Drapeau et al., 2014; Duffney et al., 2015; Lee et al., 2015; Jaramillo et al., 2016, 2017). Similarly, motor learning in accelerating rotarod was found to be impaired in $\Delta 4-9$, $\Delta 11, \Delta 13, \Delta 13-16$, and $\Delta 21$ models (Bozdagi et al., 2010; Wang et al., 2011; Yang et al., 2012; Kouser et al., 2013; Zhu et al., 2014; Speed et al., 2015; Mei et al., 2016; Jaramillo et al., 2017; Vicidomini et al., 2017) although not replicated in other studies $(\Delta 4-9, \Delta 13-16$, or $\Delta 2$; Peça et al., 2011; Drapeau et al., 2014; Duffney et al., 2015; Bidinosti et al., 2016; Jaramillo et al., 2016; Li et al., 2017). In agreement with Wang et al. (2011), both spontaneous locomotion and rotarod learning were strongly impaired in our Shank3 ${ }^{\Delta 4-22}$ mouse model. Interestingly, while most models only reported deficits in homozygous animals, heterozygous mice were also affected, albeit less se- 

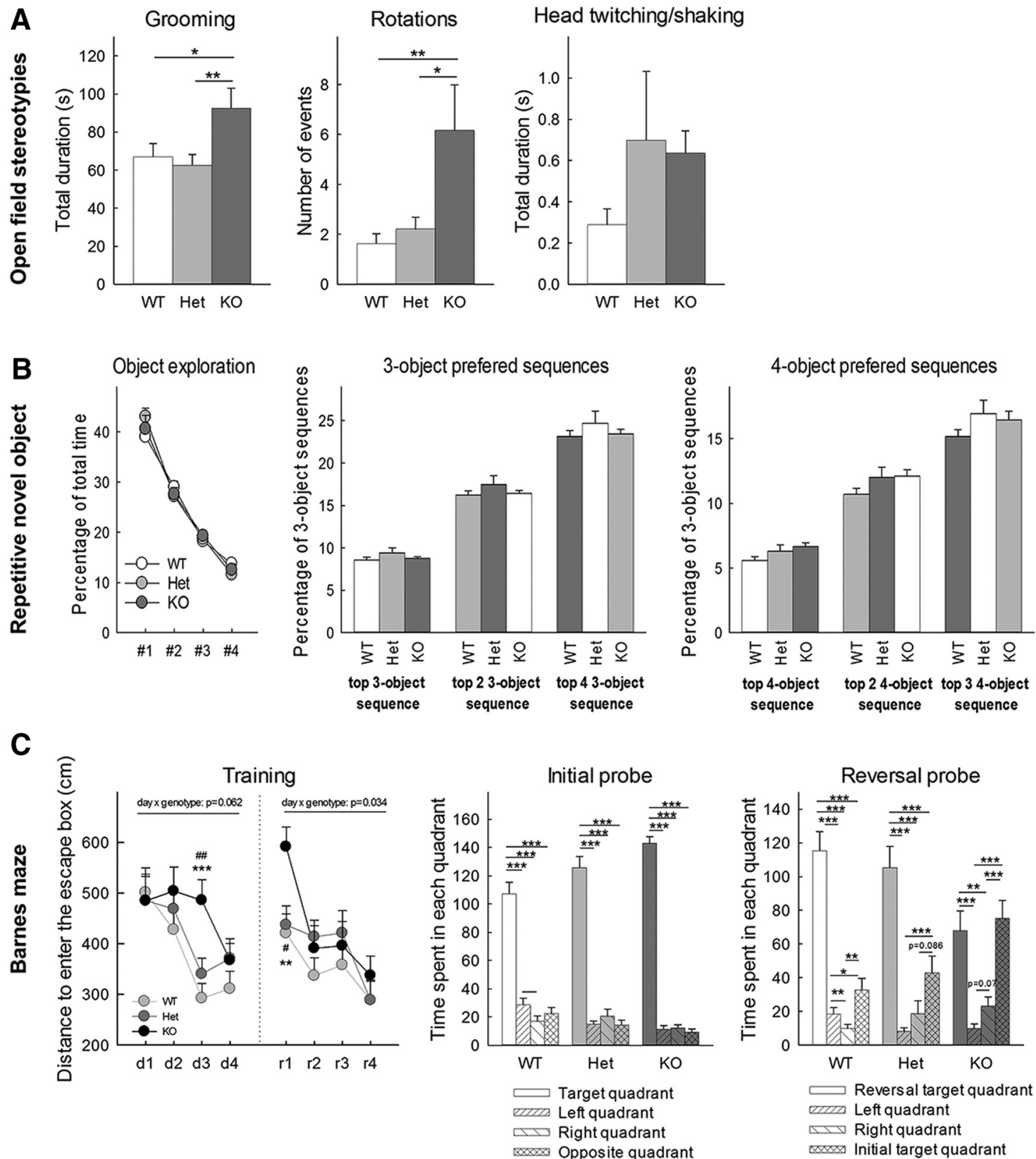

Figure 8. Repetitive behavior, stereotypies, and cognitive flexibility in Shank $3^{\Delta 4-22}$-deficient mice. $\boldsymbol{A}$, Repetitive behaviors in the open field test. Shank $3^{\Delta 4-22}$ homozygous mice engaged in significantly more self-grooming and rotations relative to the other genotypes. A trend toward an increase amount of head stereotypies was also observed. $\boldsymbol{B}$, Object preference and pattern of exploration in the repetitive novel object contact task. For each mouse, the time spent interacting with each object was measured and the objects were then ranked from the most (1) to less (4) preferred (left panel). No genotype differences were observed for the proportions of visits to each object. The pattern of object exploration was analyzed by recording specific sequential pattern of visits to three or four specific toys to identify the total number of three-object or four-object sequence investigations, the number of unique sequences, and the percentage of choices of the top, top two, or top three preferred sequences. All groups had identical percentage of their preferred three-object or four-object sequences choices over the total number of sequence choices. $\boldsymbol{C}$, Cognitive flexibility measured by reversal learning in the Barnes maze. During initial learning (d1 to $\mathrm{d} 4$, each point represents the mean of traveled distance for four independent trials), improvement shown by reduction of the travel distance was faster in Shank $3^{\Delta 4-22}$ wild-type and heterozygous mice than in homozygous animals; however, by day 4, the three groups were not different anymore and all of them had a strong preference for the escape hole quadrant during the initial probe test. During the reversal training ( $r 1$ to $\mathrm{r} 4$, each point represents the mean of travel distance for four independent trials), Shank $3^{\Delta 4-22}$ homozygous mice initially traveled for longer distances but were still able to learn the new position and performed as well as their littermates on reversal days 2,3 , and 4 . However, the reversal probe test at the end of the reversal training showed that while wild-type and heterozygous animals had a significant preference for the new target quadrant, the homozygous mice had a similar preference for the quadrants containing the initial and the reversal escape holes. WT, wild-type mice; Het, heterozygous mice; KO, homozygous knock-out mice. *: WT versus KO; \#: Het versus KO. $* p<0.05, * * p<0.1, * * * p<0.001$. 
Table 12. Detailed results and statistical analyses related to learning and memory

\begin{tabular}{|c|c|c|c|c|c|c|c|c|c|c|c|c|c|c|c|c|c|}
\hline \multicolumn{18}{|c|}{ Y-maze, spontaneous alternation behavior } \\
\hline \multirow[b]{2}{*}{$\%$ of choices } & & \multirow[b]{2}{*}{$\begin{array}{l}\text { Data } \\
\text { structure }\end{array}$} & \multirow[b]{2}{*}{ WT } & \multirow[b]{2}{*}{ Het } & \multirow[b]{2}{*}{$\mathrm{KO}$} & \multicolumn{3}{|c|}{ Genotype } & \multicolumn{3}{|l|}{ Cohort } & \multicolumn{2}{|c|}{$\begin{array}{l}\text { Genotype } \times \\
\text { cohort }\end{array}$} & \multirow[b]{2}{*}{ Power } & \multicolumn{3}{|c|}{$\begin{array}{l}\text { Pairwise } \\
\text { comparisons }\end{array}$} \\
\hline & & & & & & $F$ & $\begin{array}{l}p \\
\text { value }\end{array}$ & Power & $F$ & $\begin{array}{l}p \\
\text { value }\end{array}$ & Power & $F$ & $\begin{array}{l}p \\
\text { value }\end{array}$ & & $\begin{array}{l}\text { WT vs } \\
\text { Het }\end{array}$ & $\begin{array}{l}\text { WT vs } \\
\text { KO }\end{array}$ & $\begin{array}{l}\text { Het vs } \\
\text { KO }\end{array}$ \\
\hline Arm 1 & 2WANOVA & Normal & $32.34 \pm 0.87$ & $34.24 \pm 0.92$ & $32.77 \pm 1.17$ & 0.844 & 0.436 & 0.187 & 0.035 & 0.852 & 0.054 & 0.412 & 0.664 & 0.113 & - & - & - \\
\hline Arm 2 & 2WANOVA & Normal & $35.17 \pm 1.17$ & $32.74 \pm 1.1$ & $35.18 \pm 1.36$ & 1.548 & 0.223 & 0.314 & 9.976 & 0.003 & 0.873 & 0.119 & 0.888 & 0.067 & - & - & - \\
\hline Arm 3 & 2WANOVA & Normal & $32.19 \pm 1.46$ & $32.98 \pm 1.09$ & $32.04 \pm 1.02$ & 0.285 & 0.753 & 0.093 & 10.366 & 0.002 & 0.885 & 0.520 & 0.598 & 0.131 & - & - & - \\
\hline Chance level comparison & Test & $\begin{array}{l}\text { Data } \\
\text { structure }\end{array}$ & & & & All $t$ & $\begin{array}{l}\text { All } p \\
\text { value }\end{array}$ & Power & WT t & $\begin{array}{l}\text { WT } p \\
\text { value }\end{array}$ & Power & Het $t$ & $\begin{array}{l}\text { Het } p \\
\text { value }\end{array}$ & Power & $\mathrm{KO} \mathrm{t}$ & $\begin{array}{l}\text { KO } p \\
\text { value }\end{array}$ & Power \\
\hline Arm 1 & $1 \mathrm{~S}-t$ test & Normal & & & & Normal & Normal & Normal & Normal & Normal & Normal & Normal & Normal & Normal & Normal & Normal & Normal \\
\hline Arm 2 & $1 \mathrm{~S}-t$ test & Normal & & & & 1.465 & 0.148 & NA & 1.578 & 0.132 & NA & -0.534 & 0.600 & NA & 1.354 & 0.193 & NA \\
\hline \multirow[t]{3}{*}{ Arm 3} & $1 \mathrm{~S}-t$ test & Normal & & & & -1.338 & 0.186 & NA & -0.772 & 0.450 & NA & -0.312 & 0.759 & NA & -1.262 & 0.223 & NA \\
\hline & & & & & & \multicolumn{3}{|c|}{ Genotype } & Cohort & & & $\begin{array}{l}\text { Genotyp } \\
\text { cohort }\end{array}$ & pe $\times$ & & $\begin{array}{l}\text { Pairwise } \\
\text { compar }\end{array}$ & $\begin{array}{l}\text { e } \\
\text { isons }\end{array}$ & \\
\hline & Test & $\begin{array}{l}\text { Data } \\
\text { structure }\end{array}$ & WT & Het & KO & $F$ & $\begin{array}{l}p \\
\text { value }\end{array}$ & Power & $F$ & $\begin{array}{l}p \\
\text { value }\end{array}$ & Power & $F$ & $\begin{array}{l}p \\
\text { value }\end{array}$ & Power & $\begin{array}{l}\text { WT vs } \\
\text { Het }\end{array}$ & $\begin{array}{l}\text { WT vs } \\
\text { KO }\end{array}$ & $\begin{array}{l}\text { Het vs } \\
\text { KO }\end{array}$ \\
\hline Total number of choices & 2WANOVA & Normal & $43.42 \pm 3.25$ & $40.26 \pm 2.54$ & $38.47 \pm 2.75$ & 0.612 & 0.546 & 0.147 & 0.164 & 0.687 & 0.068 & 2.244 & 0.116 & 0.437 & - & - & - \\
\hline Number of correct choice & 2WANOVA & Normal & $57.46 \pm 1.59$ & $60.68 \pm 1.93$ & $57.52 \pm 1.61$ & 1.227 & 0.302 & 0.256 & 2.987 & 0.090 & 0.396 & 0.927 & 0.402 & 0.202 & - & - & - \\
\hline Number of type 1 errors & 2WANOVA & Normal & $37.8 \pm 1.43$ & $34.09 \pm 1.59$ & $38.29 \pm 1.79$ & 2.296 & 0.111 & 0.445 & 0.300 & 0.586 & 0.084 & 5.165 & 0.009 & 0.804 & - & - & - \\
\hline Number of type 2 errors & 2WANOVA & Nonnormal & $4.04 \pm 1.11$ & $5.47 \pm 1.02$ & $4.51 \pm 1.07$ & 0.397 & 0.674 & 0.111 & 4.402 & 0.041 & 0.539 & 3.449 & 0.039 & 0.621 & - & - & - \\
\hline Fear conditioning & & & & & & & & & & & & & & & & & \\
\hline Training & Test & $\begin{array}{l}\text { Data } \\
\text { structure }\end{array}$ & & & & $F$ & $\begin{array}{l}p \\
\text { value }\end{array}$ & Power & $\begin{array}{l}\text { WT vs } \\
\text { Het }\end{array}$ & $\begin{array}{l}\text { WT vs } \\
\text { KO }\end{array}$ & $\begin{array}{l}\text { Het vs } \\
\text { KO }\end{array}$ & & & & & & \\
\hline - Time & rMeasures & Sph.viol & & & & 43.998 & 0.000 & 1.000 & - & - & - & & & & & & \\
\hline - Time $\times$ genotype & rMeasures & Sph.viol & & & & 3.194 & 0.002 & 0.970 & - & - & - & & & & & & \\
\hline - Genotype & rMeasures & Sph.viol & & & & 14.505 & 0.000 & 0.998 & 0.809 & 0.000 & 0.000 & & & & & & \\
\hline - Cohort & rMeasures & Sph.viol & & & & 12.351 & 0.001 & 0.932 & - & - & - & & & & & & \\
\hline - Time $\times$ gen. $\times$ coh & rMeasures & Sph.viol & & & & 0.602 & 0.782 & 0.281 & - & - & - & & & & & & \\
\hline - Gen. × coh. & rMeasures & Sph.viol & & & & 0.494 & 0.613 & 0.127 & - & - & - & & & & & & \\
\hline & & & & & & Genotyp & & & Cohort & & & $\begin{array}{l}\text { Genotyp } \\
\text { cohort }\end{array}$ & pe $\times$ & & $\begin{array}{l}\text { Pairwise } \\
\text { compar }\end{array}$ & $\begin{array}{l}\text { e } \\
\text { isons }\end{array}$ & \\
\hline $\begin{array}{l}\text { Training, individual } \\
\text { time bins }\end{array}$ & Test & $\begin{array}{l}\text { Data } \\
\text { structure }\end{array}$ & WT & Het & KO & $F$ & $\begin{array}{l}p \\
\text { value }\end{array}$ & Power & $F$ & $\begin{array}{l}p \\
\text { value }\end{array}$ & Power & $F$ & $\begin{array}{l}p \\
\text { value }\end{array}$ & Power & $\begin{array}{l}\text { WT vs } \\
\text { Het }\end{array}$ & $\begin{array}{l}\text { WT vs } \\
\text { KO }\end{array}$ & $\begin{array}{l}\text { Het vs } \\
\text { KO }\end{array}$ \\
\hline Habituation & 2WANOVA & Nonnormal & $16.85 \pm 2.93$ & $10.45 \pm 2.37$ & $21.6 \pm 5.3$ & 2.081 & 0.135 & 0.408 & 0.015 & 0.903 & 0.052 & 0.061 & 0.941 & 0.059 & - & - & - \\
\hline Pre-tone $0-120$ & 2WANOVA & Nonnormal & $10.45 \pm 1.72$ & $8.15 \pm 1.43$ & $21.01 \pm 3.97$ & 6.546 & 0.003 & 0.892 & 0.330 & 0.568 & 0.087 & 0.041 & 0.960 & 0.056 & 0.820 & 0.021 & 0.004 \\
\hline Tone/shock 120-140 & 2WANOVA & Nonnormal & $10.58 \pm 3.26$ & $6.85 \pm 2.82$ & $19.94 \pm 6.06$ & 2.361 & 0.105 & 0.456 & 0.020 & 0.887 & 0.052 & 0.199 & 0.820 & 0.079 & - & - & - \\
\hline Post-tone $140-260$ & 2WANOVA & Nonnormal & $19.74 \pm 3.73$ & $18.83 \pm 3.86$ & $47.68 \pm 6.71$ & 13.149 & 0.000 & 0.996 & 5.506 & 0.023 & 0.634 & 2.222 & 0.119 & 0.433 & 0.990 & 0.000 & 0.000 \\
\hline Tone/shock 260-280 & 2WANOVA & Nonnormal & $15.07 \pm 4.7$ & $24.58 \pm 5.22$ & $47.23 \pm 7.47$ & 7.613 & 0.001 & 0.934 & 0.026 & 0.871 & 0.053 & 0.762 & 0.472 & 0.173 & 0.507 & 0.001 & 0.027 \\
\hline Tone/shock 260-280 & 2WANOVA & Nonnormal & $31.06 \pm 5.14$ & $37.88 \pm 6.6$ & $65.23 \pm 6.72$ & 12.505 & 0.000 & 0.995 & 18.006 & 0.000 & 0.986 & 0.640 & 0.532 & 0.151 & 0.650 & 0.000 & 0.002 \\
\hline Tone/shock 400-420 & 2WANOVA & Nonnormal & $31.03 \pm 5.86$ & $40.59 \pm 7.61$ & $65.21 \pm 5.94$ & 9.728 & 0.000 & 0.977 & 12.565 & 0.001 & 0.935 & 0.061 & 0.941 & 0.059 & 0.503 & 0.001 & 0.015 \\
\hline Post-tone $420-540$ & 2WANOVA & Nonnormal & $36.71 \pm 6.53$ & $47.61 \pm 7.36$ & $61.74 \pm 6.78$ & 7.880 & 0.001 & 0.942 & 45.207 & 0.000 & 1.000 & 0.135 & 0.874 & 0.070 & 0.303 & 0.003 & 0.139 \\
\hline Context & Test & $\begin{array}{l}\text { Data } \\
\text { structure }\end{array}$ & & & & $F$ & $\begin{array}{l}p \\
\text { value }\end{array}$ & Power & $\begin{array}{l}\text { WT vs } \\
\text { Het }\end{array}$ & $\begin{array}{l}\text { WT vs } \\
\text { KO }\end{array}$ & $\begin{array}{l}\text { Het vs } \\
\text { KO }\end{array}$ & & & & & & \\
\hline - Time & rMeasures & Sph.ass & & & & 4.558 & 0.004 & 0.880 & - & - & - & & & & & & \\
\hline - Time $\times$ genotype & rMeasures & Sph.ass & & & & 0.675 & 0.670 & 0.262 & - & - & - & & & & & & \\
\hline - Genotype & rMeasures & Sph.ass & & & & 1.788 & 0.178 & 0.357 & - & - & - & & & & & & \\
\hline - Cohort & rMeasures & Sph.ass & & & & 0.542 & 0.465 & 0.112 & - & - & - & & & & & & \\
\hline - Time $\times$ gen $\times$ coh . & rMeasures & Sph.ass & & & & 0.918 & 0.481 & 0.355 & - & - & - & & & & & & \\
\hline - Gen. $\times$ coh. & rMeasures & Sph.ass & & & & 1.026 & 0.366 & 0.219 & - & - & - & & & & & & \\
\hline & & & & & & Genotyp & & & Cohort & & & $\begin{array}{l}\text { Genotyp } \\
\text { cohort }\end{array}$ & pe $\times$ & & $\begin{array}{l}\text { Pairwise } \\
\text { compar }\end{array}$ & $\begin{array}{l}\text { e } \\
\text { isons }\end{array}$ & \\
\hline $\begin{array}{l}\text { Context, individual time } \\
\text { bins }\end{array}$ & Test & $\begin{array}{l}\text { Data } \\
\text { structure }\end{array}$ & WT & Het & KO & $F$ & $\begin{array}{l}p \\
\text { value }\end{array}$ & Power & $F$ & $\begin{array}{l}p \\
\text { value }\end{array}$ & Power & $F$ & $\begin{array}{l}p \\
\text { value }\end{array}$ & Power & $\begin{array}{l}\text { WT vs } \\
\text { Het }\end{array}$ & $\begin{array}{l}\text { WT vs } \\
\text { KO }\end{array}$ & $\begin{array}{l}\text { Het vs } \\
\text { KO }\end{array}$ \\
\hline $0-60$ & 2WANOVA & Nonnormal & $63.09 \pm 5.13$ & $57.19 \pm 5.63$ & $44.3 \pm 6.21$ & 2.643 & 0.081 & 0.502 & 0.582 & 0.449 & 0.116 & 0.558 & 0.576 & 0.137 & 0.750 & 0.063 & 0.261 \\
\hline $60-120$ & 2WANOVA & Nonnormal & $66.34 \pm 6.83$ & $66 \pm 6.94$ & $59.6 \pm 7.41$ & 0.230 & 0.795 & 0.084 & 0.676 & 0.415 & 0.127 & 2.233 & 0.118 & 0.435 & - & - & - \\
\hline $120-180$ & 2WANOVA & Nonnormal & $62.65 \pm 7.14$ & $62.11 \pm 6.47$ & $42.81 \pm 7.83$ & 2.263 & 0.114 & 0.440 & 0.392 & 0.534 & 0.094 & 0.958 & 0.390 & 0.207 & - & - & - \\
\hline $180-240$ & 2WANOVA & Nonnormal & $56.12 \pm 6.56$ & $54.45 \pm 7.58$ & $43.33 \pm 6.29$ & 0.944 & 0.396 & 0.205 & 0.073 & 0.788 & 0.058 & 0.109 & 0.897 & 0.066 & - & - & - \\
\hline mean & 2WANOVA & Nonnormal & $62.05 \pm 5.57$ & $59.94 \pm 5.31$ & $47.51 \pm 5.84$ & 1.788 & 0.178 & 0.357 & 0.542 & 0.465 & 0.112 & 1.026 & 0.366 & 0.219 & - & - & - \\
\hline Cued & Test & $\begin{array}{l}\text { Data } \\
\text { structure }\end{array}$ & & & & $F$ & $\begin{array}{l}p \\
\text { value }\end{array}$ & Power & $\begin{array}{l}\text { WT vs } \\
\text { Het }\end{array}$ & $\begin{array}{l}\text { WT vs } \\
\text { KO }\end{array}$ & $\begin{array}{l}\text { Het vs } \\
\text { KO }\end{array}$ & & & & & & \\
\hline - Time & rMeasures & Sph.viol & & & & 25.753 & 0.000 & 1.000 & - & - & - & & & & & & \\
\hline - Time $\times$ genotype & rMeasures & Sph.viol & & & & 3.101 & 0.002 & 0.968 & - & - & - & & & & & & \\
\hline - Genotype & rMeasures & Sph.viol & & & & 5.657 & 0.006 & 0.841 & 0.645 & 0.007 & 0.065 & & & & & & \\
\hline - Cohort & rMeasures & Sph.viol & & & & 4.255 & 0.044 & 0.525 & - & - & - & & & & & & \\
\hline - Time $\times$ gen. $\times$ coh & rMeasures & Sph.viol & & & & 4.116 & 0.000 & 0.995 & - & - & - & & & & & & \\
\hline - Gen. $\times$ coh. & rMeasures & Sph.viol & & & & $\begin{array}{l}1.616 \\
\text { ontinued) }\end{array}$ & 0.209 & 0.326 & - & - & - & & & & & & \\
\hline
\end{tabular}


Table 12. Continued

\begin{tabular}{|c|c|c|c|c|c|c|c|c|c|c|c|c|c|c|c|c|c|}
\hline \multirow[b]{2}{*}{$\begin{array}{l}\text { Cued, individual } \\
\text { time bins }\end{array}$} & \multirow[b]{2}{*}{ Test } & \multirow[b]{2}{*}{$\begin{array}{l}\text { Data } \\
\text { structure }\end{array}$} & \multirow[b]{2}{*}{ WT } & \multirow[b]{2}{*}{ Het } & \multirow[b]{2}{*}{$\mathrm{KO}$} & \multicolumn{3}{|c|}{ Genotype } & \multicolumn{3}{|l|}{ Cohort } & \multicolumn{2}{|c|}{$\begin{array}{l}\text { Genotype } \times \\
\text { cohort }\end{array}$} & \multirow[b]{2}{*}{ Power } & \multicolumn{3}{|c|}{$\begin{array}{l}\text { Pairwise } \\
\text { comparisons }\end{array}$} \\
\hline & & & & & & $F$ & $\begin{array}{l}p \\
\text { value }\end{array}$ & Power & $F$ & $\begin{array}{l}p \\
\text { value }\end{array}$ & Power & $F$ & $\begin{array}{l}p \\
\text { value }\end{array}$ & & $\begin{array}{l}\text { WT vs } \\
\text { Het }\end{array}$ & $\begin{array}{l}\text { WT vs } \\
\text { KO }\end{array}$ & $\begin{array}{l}\text { Het vs } \\
\mathrm{KO}\end{array}$ \\
\hline Pre-tone $0-60$ & 2WANOVA & Nonnormal & $1.64 \pm 1.14$ & $0.4 \pm 0.28$ & $4.9 \pm 2.59$ & 1.897 & 0.160 & 0.376 & 5.996 & 0.018 & 0.671 & 1.527 & 0.227 & 0.310 & 0.841 & 0.311 & 0.114 \\
\hline Pre-tone $60-120$ & 2WANOVA & Nonnormal & $0.84 \pm 0.38$ & $1.96 \pm 0.89$ & $2.37 \pm 0.88$ & 1.056 & 0.355 & 0.225 & 18.576 & 0.000 & 0.988 & 0.747 & 0.479 & 0.170 & 0.461 & 0.238 & 0.896 \\
\hline Tone $120-140$ & 2WANOVA & Nonnormal & $10.94 \pm 4.37$ & $12.14 \pm 4.53$ & $25.23 \pm 6.38$ & 3.005 & 0.058 & 0.558 & 7.144 & 0.010 & 0.746 & 1.298 & 0.282 & 0.268 & 0.984 & 0.106 & 0.150 \\
\hline Post-tone $140-200$ & 2WANOVA & Nonnormal & $8.52 \pm 2.11$ & $7.41 \pm 1.76$ & $13.09 \pm 3.74$ & 1.367 & 0.264 & 0.281 & 1.610 & 0.210 & 0.238 & 0.118 & 0.889 & 0.067 & - & - & - \\
\hline Post-tone $200-260$ & 2WANOVA & Nonnormal & $2.29 \pm 1.03$ & $6.59 \pm 1.53$ & $10.03 \pm 4.8$ & 1.551 & 0.222 & 0.314 & 1.595 & 0.212 & 0.236 & 1.203 & 0.309 & 0.251 & - & - & - \\
\hline Tone $260-280$ & 2WANOVA & Nonnormal & $13.87 \pm 5.15$ & $19.36 \pm 5.92$ & $39.08 \pm 7.75$ & 7.219 & 0.002 & 0.921 & 15.352 & 0.000 & 0.970 & 7.888 & 0.001 & 0.942 & 0.733 & 0.003 & 0.025 \\
\hline Post-tone $280-340$ & 2WANOVA & Nonnormal & $8.92 \pm 2.71$ & $19.61 \pm 5.1$ & $26.7 \pm 6.01$ & 3.891 & 0.027 & 0.677 & 5.448 & 0.024 & 0.629 & 1.180 & 0.316 & 0.247 & 0.240 & 0.024 & 0.527 \\
\hline Post-tone $340-400$ & 2WANOVA & Nonnormal & $5.09 \pm 1.34$ & $9.96 \pm 2.78$ & $20.99 \pm 4.81$ & 6.189 & 0.004 & 0.874 & 1.570 & 0.216 & 0.233 & 1.068 & 0.351 & 0.227 & 0.549 & 0.003 & 0.054 \\
\hline
\end{tabular}

WT, wild-type mice; Het, heterozygous mice; KO, homozygous knock-out mice. Group values are reported as mean \pm SEM. Bold font indicates significant results $(p<0.05)$. Individual results and statistical analyses for cohorts 1 and 2 are available in Extended Data Table 12-1. 2wANOVA: 2-way ANOVA, rMeasures: repeated measures, one sample t test: 1S-t test, Norm: normal, No-norm: non-normal, Sph.ass: sphericity assumed, Sph.viol: sphericity violated, gen: genotype, coh: cohort. 2wANOVA: 2-way ANOVA, rMeasures: repeated measures, one sample t test: 1S-t test, Sph.ass: sphericity assumed, Sph.viol: sphericity violated, gen: genotype, coh: cohort.

A

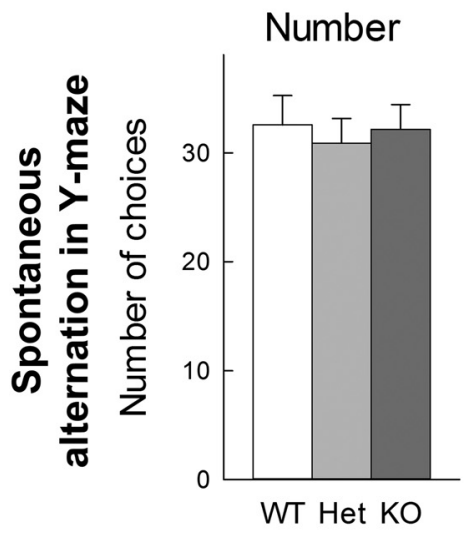

Correct choices

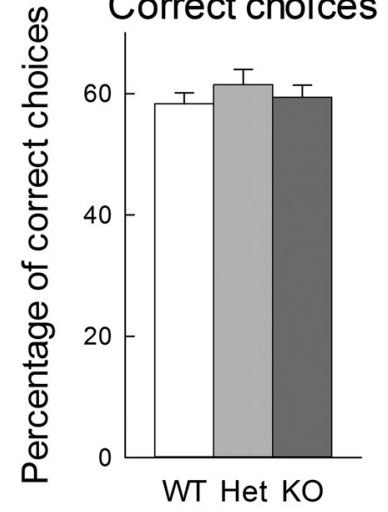

Errors 1

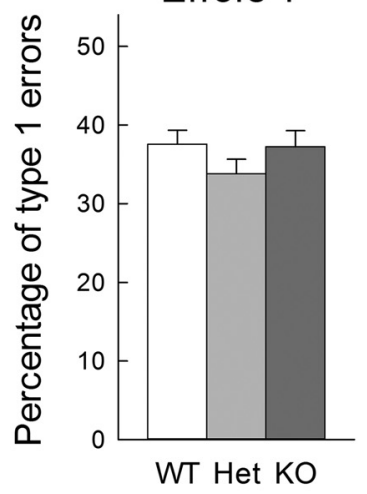

Errors 2

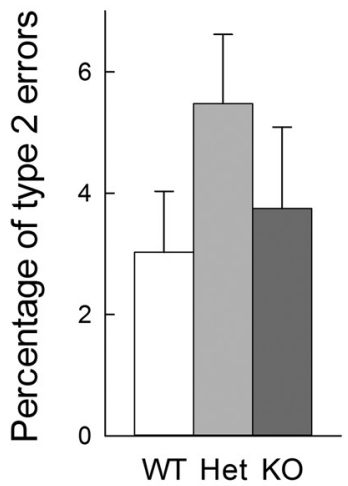

B

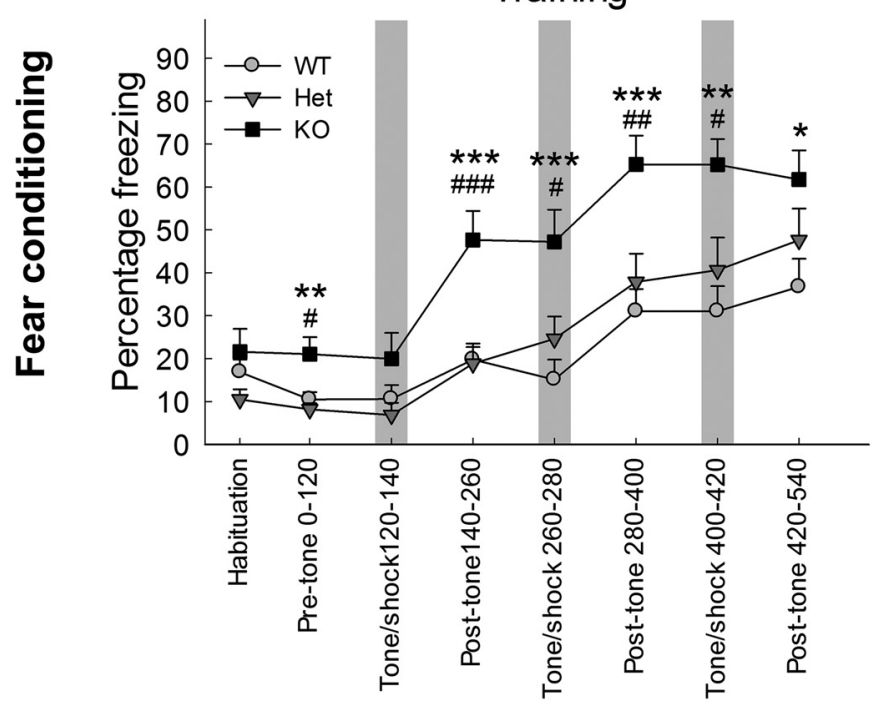

Contextual memory

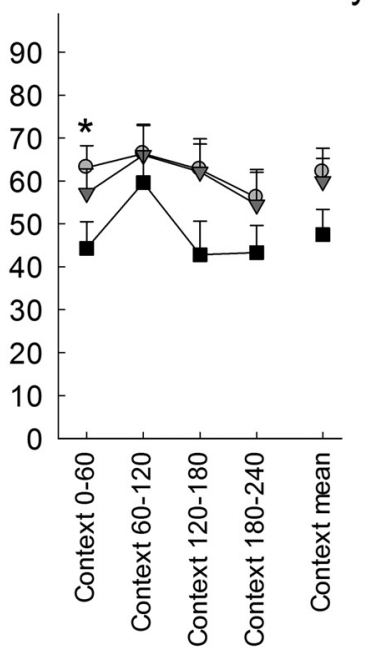

Cued memory

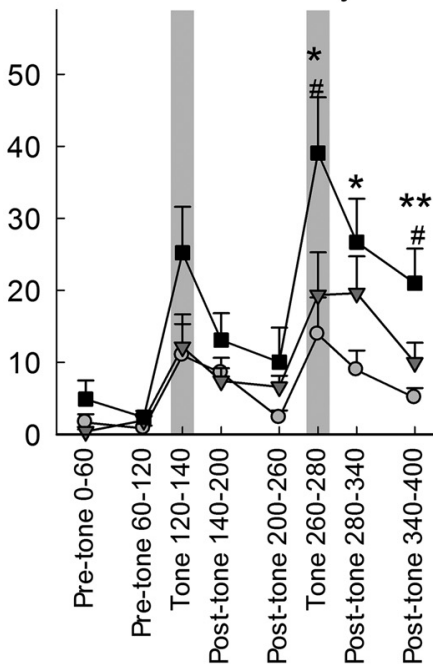

Figure 9. Learning and memory in Shank $3^{\Delta 4-22}$-deficient mice. $\boldsymbol{A}$, Working memory in Y-maze measured by spontaneous alternation behavior. All genotypes showed comparable number of arm choices, percentage of correct choices (three-way alternation), type 1 error (three consecutive choices where the first and third choices are identical), or type 2 error (three consecutive choices where the second and third choices are identical). $\boldsymbol{B}$, Contextual and cued fear conditioning in Shank3 mice. A higher percentage of freezing was observed in Shank $3^{\Delta 4-22}$ homozygous mice compared to wild-type and heterozygous animals on day 1 . While the difference was already present before the sound-shocks associations, it was strongly increased posttraining. No genotype differences were detected in freezing scores in the posttraining session on day 1. Opposite results were observed for contextual conditioning (day 2) and cued conditioning (day 3): Shank $3^{\Delta 4-22}$ homozygous mice showed an impairment of contextual learning compared to their wild-type and heterozygous littermates but an enhancement of freezing postcues during the cued testing. WT, wild-type mice; Het, heterozygous mice; KO, homozygous knock-out mice. $*$ : WT versus KO; \#: Het versus KO. $* p<0.05, * * p<0.1, * * * p<0.001$ 
Table 13. Detailed results and statistical analyses related to anxiety-like behaviors

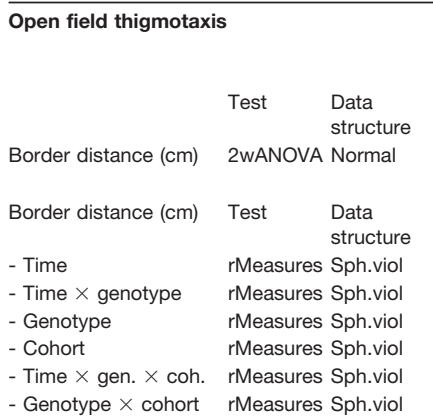

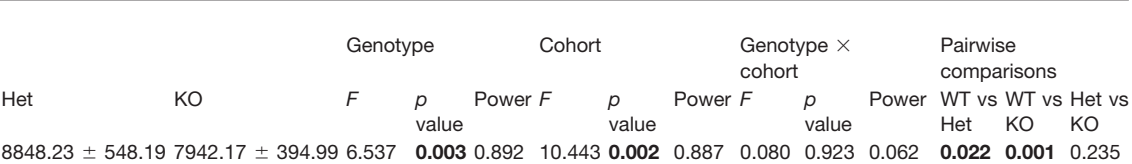

\begin{tabular}{|c|c|c|}
\hline & Test & $\begin{array}{l}\text { Data } \\
\text { structure }\end{array}$ \\
\hline Center distance (cm) & 2WANOVA & A Nonnormal \\
\hline Center distance $(\mathrm{cm})$ & Test & $\begin{array}{l}\text { Data } \\
\text { structure }\end{array}$ \\
\hline - Time & rMeasures & S Sph.viol \\
\hline - Time $\times$ genotype & rMeasures & Sph.viol \\
\hline - Genotype & rMeasures & Sph.viol \\
\hline - Cohort & rMeasures & Sph.viol \\
\hline - Time $\times$ gen. $\times$ coh . & rMeasures & Sph.viol \\
\hline - Genotype X cohort & rMeasures & Sph.viol \\
\hline
\end{tabular}

\begin{tabular}{|c|c|c|}
\hline & Test & $\begin{array}{l}\text { Data } \\
\text { structure }\end{array}$ \\
\hline Border/total distance & 2WANOVA & A Normal \\
\hline Border/total distance & Test & $\begin{array}{l}\text { Data } \\
\text { structure }\end{array}$ \\
\hline - Time & rMeasures & Sph.viol \\
\hline - Time $\times$ genotype & rMeasures & Sph.viol \\
\hline - Genotype & rMeasures & Sph.viol \\
\hline - Cohort & rMeasures & Sph.viol \\
\hline - Time $\times$ gen. $\times$ coh & rMeasures & Sph.vio \\
\hline - Genotype $\times$ cohort & rMeasures & Sph.viol \\
\hline
\end{tabular}

\begin{tabular}{|c|c|c|}
\hline & Test & $\begin{array}{l}\text { Data } \\
\text { structure }\end{array}$ \\
\hline distance & 2WANOVA & Normal \\
\hline istance & Test & $\begin{array}{l}\text { Data } \\
\text { structure }\end{array}$ \\
\hline Time & rMeasures & Sph \\
\hline Time $\times$ genotype & rMeasures & $\mathrm{Sp}$ \\
\hline Genotype & Meas & $\mathrm{Sr}$ \\
\hline - Cohort & rMeasures & Sph.viol \\
\hline - Time $\times$ gen. $\times$ & rMeasures & Sph.viol \\
\hline Genotype $\times$ cohort & rMeasures & Sph.viol \\
\hline
\end{tabular}

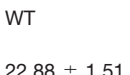

Het

$20.15 \pm 1.68$

KO

$15 \pm 1.68 \quad 20.16 \pm 1.27$

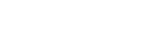

\begin{tabular}{llllll}
$F$ & $p$ & \multicolumn{3}{c}{ Power WT vs } & \multicolumn{2}{l}{ WT vs Het vs } \\
& value & Het & KO & KO \\
52.599 & 0.000 & 1.000 & - & - & - \\
2.496 & $\mathbf{0 . 0 0 7}$ & 0.877 & - & - & - \\
6.537 & $\mathbf{0 . 0 0 3}$ & 0.892 & $\mathbf{0 . 0 4 3}$ & $\mathbf{0 . 0 0 1}$ & 0.373 \\
10.443 & $\mathbf{0 . 0 0 2}$ & 0.887 & - & - & - \\
0.923 & 0.492 & 0.399 & - & - & - \\
0.080 & 0.923 & 0.062 & - & - & -
\end{tabular}

Genotype Cohort Genotype $\times \quad$ Pairwise $F \quad \begin{array}{llllll}p & \text { Power } F & p & \text { Power } F & p & \text { Power WT vs WT vs Het vs } \\ \text { value } & \text { value } & \text { value } & \text { Het KO KO }\end{array}$

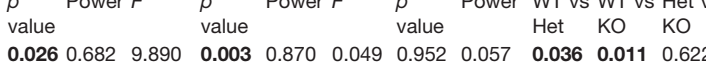

F $\quad p \quad$ Power WT vs WT vs Het vs value Het KO KO

$\begin{array}{lllllllll}1.158 & 0.330 & 0.343 & - & - & -\end{array}$

$1.327 \quad 0.2370 .571-\quad-\quad-$

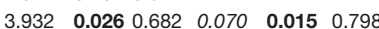

$\begin{array}{llllllll}9.890 & 0.003 & 0.870 & - & - & -\end{array}$

$0.6950 .6830 .302-\quad-\quad-$

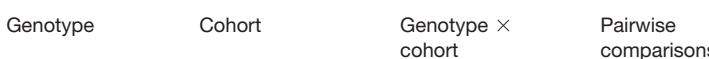

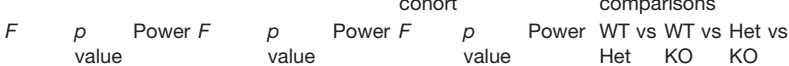
$\begin{array}{llllllllllll}0.950 & 0.393 & 0.206 & 5.570 & 0.022 & 0.639 & 0.049 & 0.952 & 0.057 & - & - & -\end{array}$

F $\quad p \quad$ Power WT vs WT vs Het vs value Het KO KO

$\begin{array}{llllll}5.035 & 0.001 & 0.957- & - & -\end{array}$

$\begin{array}{llllll}1.182 & 0.312 & 0.531 & - & - & -\end{array}$

$\begin{array}{llll}1.017 & 0.369 & 0.218 & -\end{array}$

$\begin{array}{lllll}5.820 & 0.019 & 0.658 & - & -\end{array}$

$0.6790 .7050 .305-\quad-$

$0.0940 .9110 .064-\quad-\quad-$

Genotype $\quad$ Cohort $\quad$ Genotype $\times \quad$ Pairwise

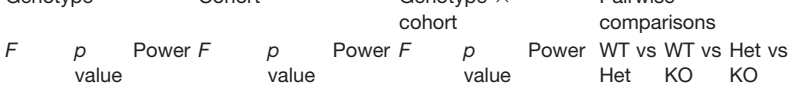
$\begin{array}{lllllllllll}0.939 & 0.398 & 0.204 & 4.471 & 0.039 & 0.546 & 0.048 & 0.953 & 0.057 & - & -\end{array}$

F $\quad p \quad$ Power WT vs WT vs Het vs

value Het KO KO

$\begin{array}{llllll}5.177 & 0.001 & 0.962 & - & - & -\end{array}$

$\begin{array}{lllll}1.177 & 0.315 & 0.527 & - & -\end{array}$

$\begin{array}{llllll}1.001 & 0.375 & 0.215 & - & - & -\end{array}$

$\begin{array}{llllll}4.757 & 0.034 & 0.571 & - & - & -\end{array}$

$\begin{array}{llllll}0.652 & 0.728 & 0.292-\quad-\quad-\end{array}$

$0.0880 .9160 .063-$

Genotype Cohort Genotype $\times$ cohort Pairwise

comparisons value Power $F \quad p \quad$ Power WT vs WT vs Het vs Het KO

$4.87+0.74 \quad 4.34+0.36$ $0.990 \quad 0.3790 .213 \quad 3.522 \quad 0.066 \quad 0.453 \quad 0.216 \quad 0.807 \quad 0.082 \quad$ Het KO KO

F $\quad$ p Power WT vs WT vs Het vs

value Het KO KO

$\begin{array}{llllll}5.177 & 0.001 & 0.240 & - & - & -\end{array}$

$\begin{array}{llll}1.177 & 0.315 & 0.456 & -\end{array}$

$\begin{array}{lll}1.001 & 0.375 & 0.309-\end{array}$

$\begin{array}{lll}4.757 & 0.034 & 0.469\end{array}$

$0.6520 .7280 .196-$

$0.088 \quad 0.9160 .230-$

Genotype Cohort Genotype $\times \quad$ Pairwise cohort comparisons

$F \quad p \quad$ Power $F \quad p \quad$ Power $F \quad p \quad$ Power WT vs WT vs Het vs value value value Het $\mathrm{KO} \mathrm{KO}$ $\begin{array}{llllllllllllll}0.481 & 0.621 & 0.124 & 1.088 & 0.302 & 0.176 & 0.582 & 0.563 & 0.141 & - & - & -\end{array}$

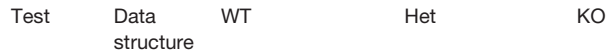

2WANOVA Normal 
Table 13. Continued

\begin{tabular}{|c|c|c|}
\hline Border time (s) & Test & $\begin{array}{l}\text { Data } \\
\text { structure }\end{array}$ \\
\hline - Time & rMeasures & Sph.viol \\
\hline - Time $\times$ genotype & rMeasures & Sph.viol \\
\hline - Genotype & rMeasures & Sph.viol \\
\hline - Cohort & rMeasures & Sph.viol \\
\hline - Time $\times$ gen. $\times$ coh. & rMeasures & Sph.viol \\
\hline - Genotype $\times$ cohort & rMeasures & Sph.viol \\
\hline
\end{tabular}

Center time (s)

Center time (s)

- Time

- Time $\times$ genotype

- Genotype

- Cohort

- Time $\times$ gen. $\times$ coh.

- Genotype $\times$ cohort

$\begin{array}{ll}\text { Test } & \begin{array}{l}\text { Data } \\ \text { structure } \\ \text { 2wANOVA }\end{array} \\ \text { Normal } \\ \text { Test } & \begin{array}{l}\text { Data } \\ \text { structure }\end{array} \\ \text { rMeasures } & \text { Sph.viol } \\ \text { rMeasures } & \text { Sph.viol } \\ \text { rMeasures } & \text { Sph.viol } \\ \text { rMeasures } & \text { Sph.viol } \\ \text { rMeasures } & \text { Sph.viol } \\ \text { rMeasures } & \text { Sph.viol }\end{array}$

Test

Data WT
structure

Border/center time

Border/center time

- Time

- Time $\times$ genotype

- Genotype

- Cohort

- Time $\times$ gen. $\times$ coh.

- Genotype $\times$ cohort

\section{structure}

2WANOVA Nonnormal $6.95 \pm 1.32$

$\begin{array}{ll}\text { Test } & \begin{array}{l}\text { Data } \\ \text { structure }\end{array} \\ \text { rMeasures } & \text { Sph.viol } \\ \text { rMeasures } & \text { Sph.viol } \\ \text { rMeasures } & \text { Sph.viol } \\ \text { rMeasures } & \text { Sph.viol } \\ \text { rMeasures } & \text { Sph.viol } \\ \text { rMeasures } & \text { Sph.viol }\end{array}$

Vertical activity in open field

Free rears duration (s) Free rears number Wall rears duration (s) Wall rears number All rears duration (s) All rears number

$\begin{array}{llllll}F & p & \text { Power WT vs } & \text { WT vs Het vs } \\ & \text { value } & & \text { Het } & \text { KO } & \text { KO } \\ 2.960 & 0.023 & 0.773 & - & - & - \\ 0.836 & 0.568 & 0.374 & - & - & - \\ 0.481 & 0.621 & 0.124 & - & - & - \\ 1.088 & 0.302 & 0.176 & - & - & - \\ 0.792 & 0.606 & 0.354 & - & - & - \\ 0.582 & 0.563 & 0.141 & - & - & -\end{array}$

Genotype Cohort $F \quad p_{\text {value }}$ Power $F$

$F$

$p$ Power
value

Pairwise cohort comparisons $\begin{array}{llll}p & \text { Power WT vs } & \text { WT vs Het vs } \\ \text { value } & \text { Het } & \text { KO KO }\end{array}$
F $\quad p \quad$ Power WT vs WT vs Het vs $\begin{array}{llll}p & \text { Power } & \text { WT vs } & \text { WT vs } \\ \text { value } & \text { Het } & \text { KO } & \text { KO }\end{array}$ $\begin{array}{lllllllllll}3.200 & 0.016 & 0.807 & - & - & -\end{array}$ $\begin{array}{lllllll}0.836 & 0.568 & 0.363 & - & - & -\end{array}$

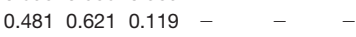
$\begin{array}{llllll}1.088 & 0.302 & 0.150 & - & - & -\end{array}$ $\begin{array}{llllll}0.792 & 0.606 & 0.090 & - & - & - \\ 0.582 & 0.563 & 0.143 & - & - & -\end{array}$

Genotype Cohort Genotype $\times$ Pairwise $F \quad$\begin{tabular}{llllll}
$p$ & \multicolumn{4}{c}{ cohort } & \multicolumn{2}{c}{ comparisons } \\
value & Power $F$ & $p$ & Power $F$ & $p$ & Power WT vs WT vs Het vs \\
value & & & value & Het KO KO
\end{tabular} $\begin{array}{lllllllllllll}0.476 & 0.624 & 0.124 & 0.533 & 0.469 & 0.111 & 0.107 & 0.898 & 0.066 & - & - & -\end{array}$

$\begin{array}{llll}p & \text { Power WT vs } & \text { WT vs Het vs } \\ \text { value } & \text { Het } & \text { KO KO }\end{array}$

$\begin{array}{llllll}0.290 & 0.822 & 0.103 & - & - & -\end{array}$

$\begin{array}{llllll}1.575 & 0.163 & 0.575 & - & - & -\end{array}$

$0.4290 .6530 .116 \quad-\quad-\quad-$

$\begin{array}{lllllll}1.546 & 0.220 & 0.230 & - & - & -\end{array}$

$\begin{array}{llllll}0.575 & 0.740 & 0.219 & - & - & - \\ 1.594 & 0.213 & 0.322 & - & - & -\end{array}$

Genotype Cohort $\quad$ Genotype $\times \quad$ Pairwise

$F$ cohort comparisons KO

$6.18 \pm 1.48$ $7.57 \pm 1.21$ $9.01 \pm 0.84$ $19.36 \pm 1.88$ $15.2 \pm 1.99$ $26.94 \pm 2.08$

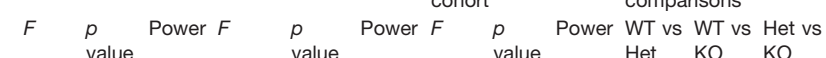
$\begin{array}{llllllllllllllll}1.159 & 0.322 & 0.243 & 0.036 & 0.850 & 0.054 & 1.480 & 0.237 & 0.301 & - & & & \end{array}$ $\begin{array}{llllllllllll}0.837 & 0.439 & 0.186 & 1.988 & 0.165 & 0.283 & 1.369 & 0.264 & 0.281 & -\end{array}$

$\begin{array}{llllllllllll}5.023 & 0.010 & 0.793 & 1.924 & 0.171 & 0.275 & 0.397 & 0.675 & 0.111 & 0.805 & \mathbf{0 . 0 4 5} & \mathbf{0 . 0 0 9}\end{array}$ $\begin{array}{lllllllllllll}3.576 & 0.035 & 0.638 & 19.306 & 0.000 & 0.991 & 0.414 & 0.663 & 0.113 & 0.996 & 0.036 & 0.030\end{array}$ $\begin{array}{llllllllllllll}3.140 & 0.052 & 0.578 & 0.646 & 0.425 & 0.124 & 1.240 & 0.298 & 0.258 & 0.374 & 0.468 & 0.038\end{array}$

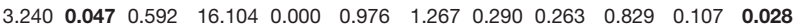

Het

$7.93 \pm 1.62$ $10.63 \pm 1.66$ $16.13 \pm 2.02$ $27.52 \pm 2.86$ $24.07 \pm 3.01$ $38.15 \pm 3.94$
Zero-maze

Closed arc time, d1 Closed arc time, d2 Closed arc time, $\mathrm{m}$ Open arc time, $d 1$ Open arc time, $\mathrm{d} 2$ Open arc time, $\mathrm{m}$ Close/open time, $\mathrm{d} 1$ Close/open time, d2 Close/open time, $\mathrm{m}$ Open arc entries, d1 Open arc entries, $\mathrm{d} 2$ Open arc entries, $m$ Open entering arc latency Open arc crossing latency Close arc dipping number, d2 2WANOVA Nonnormal $31.94 \pm 3.01$ Close arc dipping number, $m$ 2WANOVA Nonnormal $41.84 \pm 4.31$ KO

\section{Genotype Cohort}

$$
F
$$
Power $F$ value
Genotype $\times$ cohort

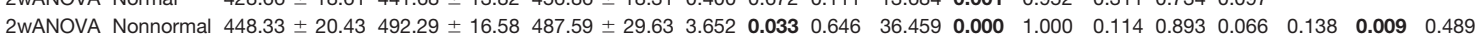
$\begin{array}{lllllllllllllll}2 \text { WANOVA Normal } & 438.5 \pm 18.27 & 466.99 \pm 13.34 & 472.23 \pm 20.15 & 1.917 & 0.158 & 0.379 & 28.873 & 0.000 & 1.000 & 0.253 & 0.778 & 0.088 & -\end{array}$

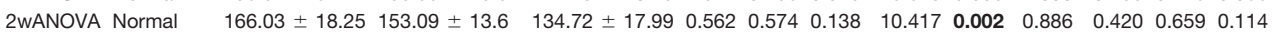

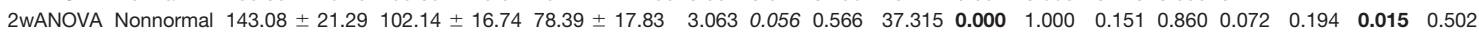

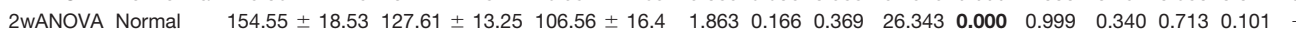
$\begin{array}{lllllllllllll}\text { 2WANOVA Nonnormal } & 4.08 \pm 0.85 & 3.74 \pm 0.98 & 5.76 \pm 1.6 & 0.820 & 0.447 & 0.182 & 9.172 & 0.004 & 0.843 & 0.438 & 0.648 & 0.117\end{array}$

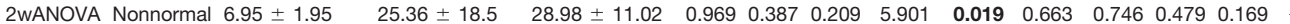
2WANOVA Nonnormal $4.61 \pm 1$

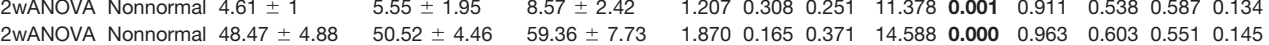

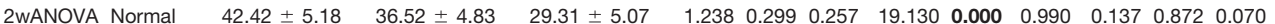
$\begin{array}{lllllllllllll}\text { 2WANOVA Normal } & 45.44 \pm 4.16 & 43.52 \pm 4.04 & 44.34 \pm 5.81 & 0.150 & 0.861 & 0.072 & 24.720 & 0.000 & 0.998 & 0.447 & 0.642 & 0.119 \\ \text { 2WANOVA Nonnormal } & 39.29 \pm 13.16 & 47.08 \pm 31.35 & 22.99 \pm 10.38 & 0.310 & 0.735 & 0.097 & 0.684 & 0.412 & 0.128 & 1.282 & 0.287 & 0.265\end{array}$

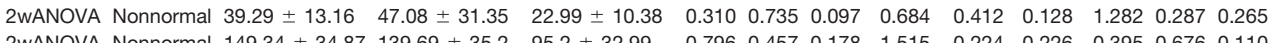
$\begin{array}{llllllllllll}139.69 \pm 35.2 & 95.2 \pm 32.99 & 0.796 & 0.457 & 0.178 & 1.515 & 0.224 & 0.226 & 0.395 & 0.676 & 0.110 \\ 55.42 \pm 6.48 & 51.47 \pm 7.31 & 0.303 & 0.740 & 0.096 & 54.807 & 0.000 & 1.000 & 0.035 & 0.965 & 0.055\end{array}$ $\begin{array}{lllllllllllll}55.42 \pm 6.48 & 51.47 \pm 7.31 & 0.303 & 0.740 & 0.096 & 54.807 & 0.000 & 1.000 & 0.035 & 0.965 & 0.055 \\ 28.94 \pm 3.69 & 22.21 \pm 3.87 & 2.182 & 0.124 & 0.425 & 54.920 & 0.000 & 1.000 & 0.593 & 0.556 & 0.143\end{array}-$ $\begin{array}{lllllllllll}42.5 \pm 4.48 & 36.84 \pm 5.05 & 0.239 & 0.788 & 0.085 & 94.671 & 0.000 & 1.000 & 0.179 & 0.836 & 0.076\end{array}$ $\begin{array}{lllllllllllllllll} & & 0.984 & 0.381 & 0.211 & 57.892 & 0.000 & 1.000 & 0.648 & 0.527 & 0.153\end{array}$ $\begin{array}{llllllllllllllll}\text { Close arc dipping time, d2 } \quad \text { 2wANOVA Nonnormal } 94.51 \pm 10 & 100.08 \pm 13.98 & 64.33 \pm 12.46 & 2.111 & 0.132 & 0.413 & 26.004 & 0.000 & 0.999 & 0.342 & 0.712 & 0.102\end{array}$

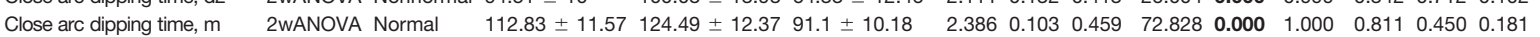
$\begin{array}{llllllllllll}\text { Open arc dipping number, d1 } 2 \text { wANOVA Nonnormal } 27.15 \pm 3.63 & 20.26 \pm 2.46 & 19.15 \pm 3.92 & 1.441 & 0.247 & 0.294 & 8.140 & 0.006 & 0.799 & 0.567 & 0.571 & 0.139\end{array}$ Open arc dipping number, d2 2 WANOVA Nonnormal $16.94 \pm 3.04 \quad 12.22 \pm 2.34 \quad 8.84 \pm 2.54$ Open arc dipping number, $m$ 2WANOVA Nonnormal $22.05 \pm 3.15$ $16.97 \pm 2.15 \quad 14 \pm 2.9$ $\begin{array}{llllllllll}1.938 & 0.155 & 0.383 & 17.131 & 0.000 & 0.982 & 0.332 & 0.719 & 0.100\end{array}$ $\begin{array}{lllllllll}2.060 & 0.138 & 0.404 & 15.216 & 0.000 & 0.969 & 0.502 & 0.609 & 0.128\end{array}$ 
Table 13. Continued

\begin{tabular}{|c|c|c|c|c|c|c|c|c|c|c|c|c|c|c|c|c|c|}
\hline \multicolumn{18}{|l|}{ Zero-maze } \\
\hline & \multirow[b]{2}{*}{ Test } & \multirow[b]{2}{*}{$\begin{array}{l}\text { Data } \\
\text { structure }\end{array}$} & \multirow[b]{2}{*}{ WT } & \multirow[b]{2}{*}{ Het } & \multirow[b]{2}{*}{ KO } & \multicolumn{2}{|l|}{ Genotype } & \multicolumn{3}{|c|}{ Cohort } & \multirow[b]{2}{*}{ Power } & \multicolumn{2}{|c|}{$\begin{array}{l}\text { Genotype } \times \\
\text { cohort }\end{array}$} & \multirow[b]{2}{*}{ Power } & \multicolumn{2}{|c|}{$\begin{array}{l}\text { Pairwise } \\
\text { comparisons }\end{array}$} & \multirow[b]{2}{*}{$\begin{array}{l}\text { Het vs } \\
\text { KO }\end{array}$} \\
\hline & & & & & & $F$ & $\begin{array}{l}p \\
\text { value }\end{array}$ & Power & $F$ & $\begin{array}{l}p \\
\text { value }\end{array}$ & & $F$ & $\begin{array}{l}p \\
\text { value }\end{array}$ & & $\begin{array}{l}\text { WT vs } \\
\text { Het }\end{array}$ & $\begin{array}{l}\text { WT vs } \\
\text { KO }\end{array}$ & \\
\hline Open arc dipping time, d1 & 2WANOVA & Nonnormal & $62.85 \pm 10.83$ & $42.28 \pm 5.15$ & $25.57 \pm 5.27$ & 5.700 & 0.006 & 0.843 & 13.928 & 0.000 & 0.955 & 1.730 & 0.188 & 0.346 & 0.091 & 0.001 & 0.245 \\
\hline Open arc dipping time, d2 & 2WANOVA & Nonnormal & $51.3 \pm 10.52$ & $35.3 \pm 8.44$ & $17.87 \pm 5.34$ & 3.798 & 0.029 & 0.665 & 20.104 & 0.000 & 0.993 & 0.427 & 0.655 & 0.115 & 0.278 & 0.008 & 0.269 \\
\hline Open arc dipping time, $\mathrm{m}$ & 2WANOVA & Nonnormal & $57.07 \pm 9.63$ & $39.17 \pm 5.86$ & $21.72 \pm 4.71$ & 6.448 & 0.003 & 0.886 & 23.422 & 0.000 & 0.997 & 1.299 & 0.282 & 0.268 & 0.088 & 0.001 & 0.157 \\
\hline Time open vs close, d1 & Test & $\begin{array}{l}\text { Data } \\
\text { structure }\end{array}$ & & & & All $F$ & $\begin{array}{l}\text { All } p \\
\text { value }\end{array}$ & Power & WT $F$ & $\begin{array}{l}\text { WT } p \\
\text { value }\end{array}$ & Power & Het $F$ & $\begin{array}{l}\text { Het } p \\
\text { value }\end{array}$ & Power & KO F & $\begin{array}{l}\text { KO } p \\
\text { value }\end{array}$ & Power \\
\hline - Zone & rMeasures & Sph.ass & & & & 277.319 & 0.000 & 1.000 & 73.861 & 0.000 & 1.000 & 125.301 & 0.000 & 1.000 & 83.255 & 0.000 & 1.000 \\
\hline - Cohort & rMeasures & Sph.ass & & & & 8.156 & 0.006 & 0.935 & 5.563 & 0.031 & 0.604 & 8.257 & 0.011 & 0.773 & 3.298 & 0.087 & 0.403 \\
\hline - Zone $\times$ cohort & rMeasures & Sph.ass & & & & 12.518 & 0.001 & 0.801 & 7.248 & 0.015 & 0.718 & 3.063 & 0.098 & 0.379 & 2.143 & 0.161 & 0.282 \\
\hline Time open vs close, d2 & Test & $\begin{array}{l}\text { Data } \\
\text { structure }\end{array}$ & & & & All $F$ & $\begin{array}{l}\text { All } p \\
\text { value }\end{array}$ & Power & WT $F$ & $\begin{array}{l}\text { WT } p \\
\text { value }\end{array}$ & Power & Het $F$ & $\begin{array}{l}\text { Het } p \\
\text { value }\end{array}$ & Power & KO F & $\begin{array}{l}\text { KO } p \\
\text { value }\end{array}$ & Power \\
\hline - Zone & rMeasures & Sph.ass & & & & 440.281 & 0.000 & 1.000 & 94.767 & 0.000 & 1.000 & 278.317 & 0.000 & 1.000 & 119.843 & 0.000 & 1.000 \\
\hline - Cohort & rMeasures & Sph.ass & & & & 0.578 & 0.450 & 0.218 & 1.269 & 0.276 & 0.186 & 2.497 & 0.132 & 0.320 & 0.921 & 0.351 & 0.148 \\
\hline - Zone $\times$ cohort & rMeasures & Sph.ass & & & & 25.848 & 0.000 & 1.000 & 12.054 & 0.003 & 0.905 & 18.624 & 0.000 & 0.982 & 3.749 & 0.070 & 0.447 \\
\hline Time open vs close, $\mathrm{m}$ & Test & $\begin{array}{l}\text { Data } \\
\text { structure }\end{array}$ & & & & All $F$ & $\begin{array}{l}\text { All } p \\
\text { value }\end{array}$ & Power & WT $F$ & $\begin{array}{l}\text { WT } p \\
\text { value }\end{array}$ & Power & Het $F$ & $\begin{array}{l}\text { Het } p \\
\text { value }\end{array}$ & Power & KO F & $\begin{array}{l}\text { KO } p \\
\text { value }\end{array}$ & Power \\
\hline - Zone & rMeasures & Sph.ass & & & & 440.281 & 0.000 & 1.000 & 103.409 & 0.000 & 1.000 & 274.392 & 0.000 & 1.000 & 131.582 & 0.000 & 1.000 \\
\hline - Cohort & rMeasures & Sph.ass & & & & 0.578 & 0.450 & 0.116 & 0.006 & 0.941 & 0.051 & 0.422 & 0.524 & 0.094 & 0.484 & 0.496 & 0.101 \\
\hline - Zone $\times$ cohort & rMeasures & Sph.ass & & & & 25.848 & 0.000 & 0.999 & 11.720 & 0.003 & 0.897 & 12.720 & 0.002 & 0.919 & 3.786 & 0.068 & 0.451 \\
\hline
\end{tabular}

WT, wild-type mice; Het, heterozygous mice; KO, homozygous knock-out mice. Group values are reported as mean \pm SEM. Bold font indicates significant results $(p<0.05)$. Individual results and statistical analyses for cohorts 1 and 2 are available in Extended Data Table 13-1. 2wANOVA: 2-way ANOVA, rMeasures: repeated measures, Norm: normal, No-norm: non-normal, Sph.ass: sphericity assumed, Sph.viol: sphericity violated, gen: genotype, coh: cohort, d1: day 1, d2: day 2, m: day 1 - day 2 mean. 2wANOVA: 2-way ANOVA, rMeasures: repeated measures, Sph.ass: sphericity assumed, Sph.viol: sphericity violated, gen: genotype, coh: cohort, d1: day 1, d2: day 2, m: day 1 - day 2 mean.

verely. Difficulties in fine motor coordination have been described in $\Delta 4-9$ and $\Delta 11$ Shank3-deficient mice (Wang et al., 2011; Drapeau et al., 2014; Vicidomini et al., 2017) and were confirmed in the current study. In addition, our homozygous mice were strongly impaired in the hanging test, the hindlimb placing test and the inverted screen and had small gait abnormalities.

Hypersensitivity or hyposensitivity to sensory stimuli is frequently observed in PMS and ASD patients (Klintwall et al., 2011; Phelan and Betancur, 2011). However, little was known regarding the sensory abilities of Shank3deficient mice. No deficits were reported in $\Delta 4-9$ or $\Delta 4-22$ animals for either olfaction, audition, vision, neuromuscular reflexes or pain sensitivity (Bozdagi et al., 2010; Wang et al., 2011, 2016b; Yang et al., 2012). Normal pre-pulse inhibition was observed in many models including $\Delta 4-9$, $\Delta 13, \Delta 21$, and $\Delta 4-22$ Shank3-deficient mice (Yang et al., 2012; Kouser et al., 2013; Wang et al., 2016b; Jaramillo et al., 2017) even if decreased pre-pulse inhibition was reported in in lines with point mutations in exon 21 (Zhou et al., 2016). Here, we observed that Shank3 $3^{\Delta 4-22}$ homozygous mice have no strong visual deficits, and normal neuromuscular reflexes, but are hyper-reactive in response to handling and tactile stimuli. In addition, we observed a delay in the acquisition of the startle response in newborns and a decrease of the startle response in both heterozygous and homozygous adults. Since social behavior strongly relies on olfaction in rodents, we used different behavioral paradigms to evaluate our model. Interestingly, Shank $3^{\Delta 4-22}$ homozygous mice had a low interest for nonsocial olfactory stimuli as shown by deficits in the buried food test and by low amount of sniffing during the olfactory habituation/dishabituation paradigm. However, Shank3 ${ }^{4-22}$-deficient mice were able to discriminate odors in the test for social transmission of food preference or to show interest for social stimuli during olfactory habituation/dishabituation, suggesting that they do not have anosmia but rather show reduced interest in nonsocial scents, which can be overcome when adding a social component.

One of the defining features of autism is the impairment of social interactions that can manifest by deficits in social approach, reciprocal social interactions and/or verbal and nonverbal communication. Mild social deficits have been reported, however with variability, in some of the previous studies of PMS mouse models (Fig. 11). In one of the most commonly used test, the three-chambered social approach test, no differences between the genotypes were reported in $\Delta 4-9, \Delta 4-7$, and $\Delta 9$ models (Peça et al., 2011; Yang et al., 2012; Drapeau et al., 2014; Lee et al., 2015), while social deficits characterized by a lack of preference for a social stimulus were reported the models targeting $\Delta 11, \Delta 13$, or $\Delta 13-16$ deletions (Peça et al., 2011; Duffney et al., 2015; Mei et al., 2016; Jaramillo et al., 2017; Luo et al., 2017; Vicidomini et al., 2017). Conflicting results were reported for $\Delta 21$ models (Kouser et al., 2013; Duffney et al., 2015; Speed et al., 2015; Bidinosti et al., 2016; Zhou et al., 2016). Interestingly, consistent with Wang et al., 2016b and colleagues' study, we observed only minimal social deficit in our $\Delta 4-22$ model. All genotypes had a similar preference for social stimulus in the three-chambered social approach test or the social transmission of food preference and only trends toward a decrease of interaction time and vocalization were found during male-female social interactions. Rodent social behavior is highly influenced by experimental conditions such as the animals' age, housing conditions, or animals handling and that can explain differences observed between cohorts of animals with identical or similar alterations of the Shank3 gene. While not representative of 
A

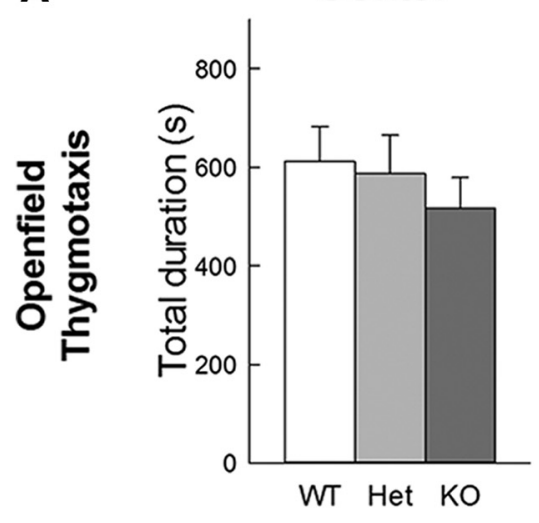

B

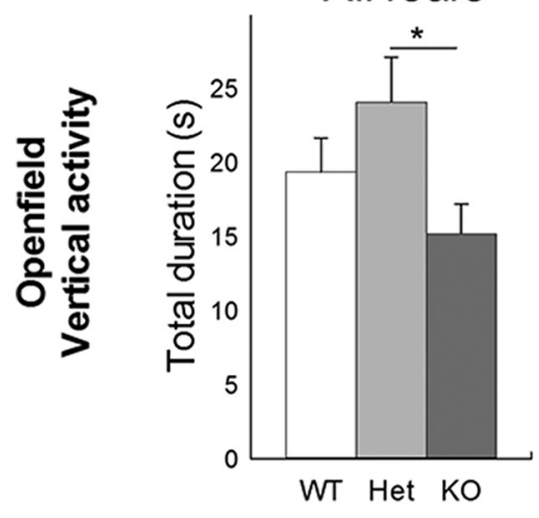

Borders
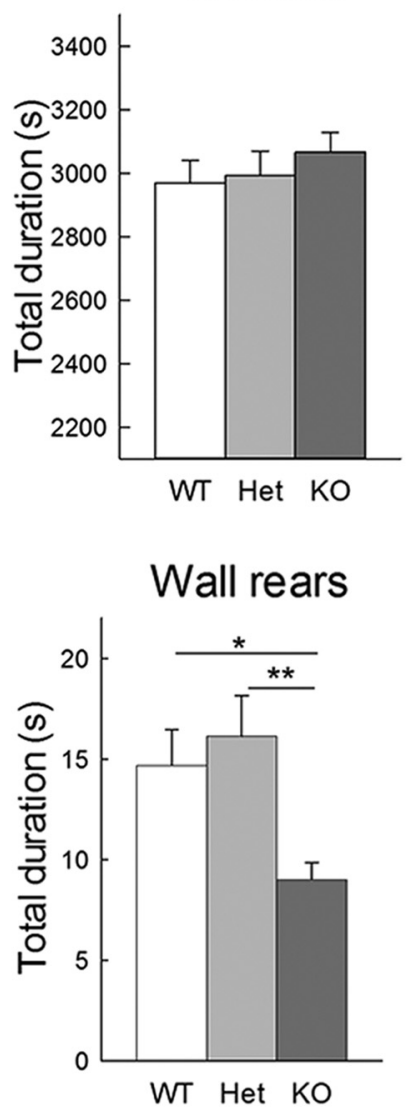

\section{Borders/center}

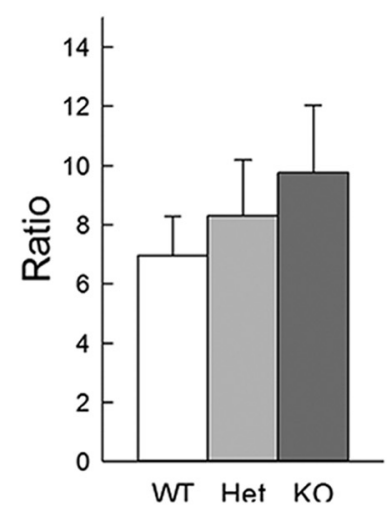

Free rears

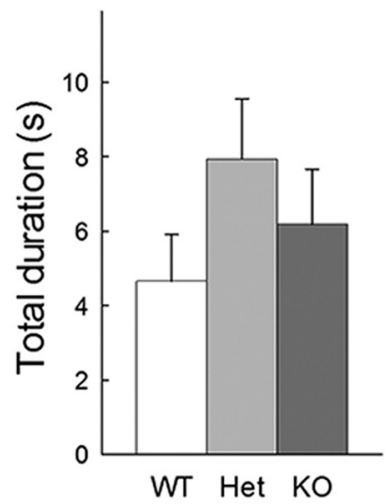

C Time spent in open arcs
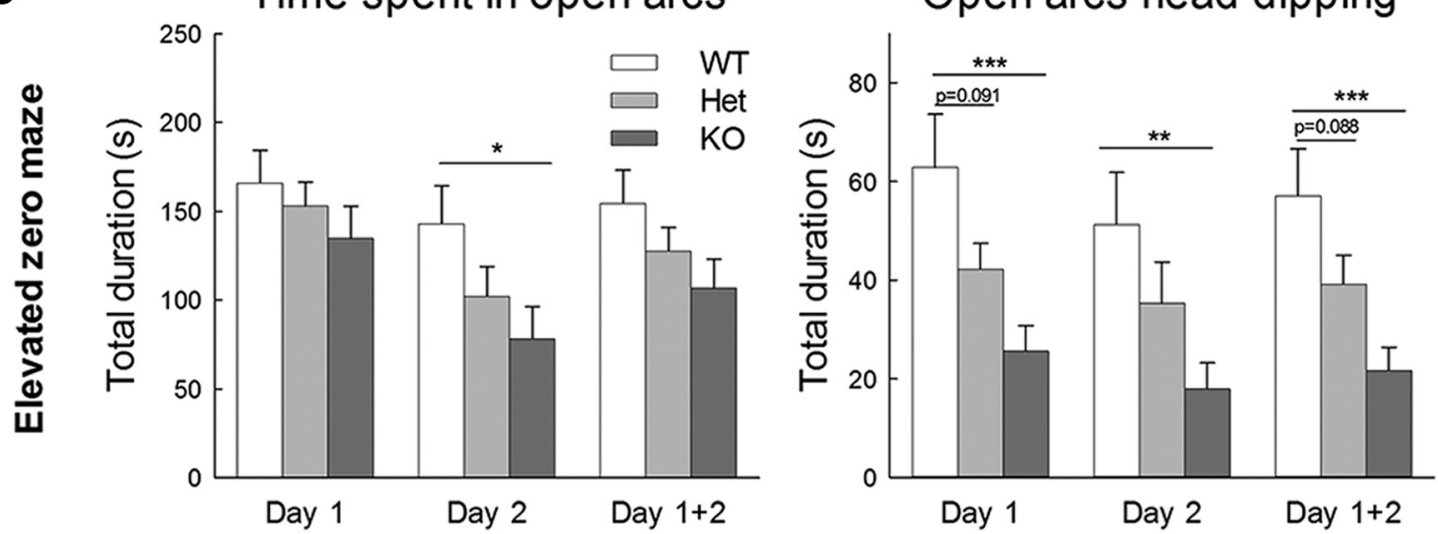

Figure 10. Anxiety-like behavior in Shank $3^{\Delta 4-22}$-deficient mice. A, Thigmotaxic behavior in open field. No genotype differences were found for the time spent in the center of the open field, the time spent close to the chamber walls (borders), or their ratio. $\boldsymbol{B}$, Vertical activity in open field. The cumulated time spend in free standing rears and rears against the walls of the open field were both counted. When compared to wild-type and heterozygotes littermates, Shank $3^{\Delta 4-22}$ homozygous mice displayed decreased rearing activity due to a decrease of wall rears rather than free standing rears. $C$, Shank $3^{\Delta 4-22}$ homozygous mice spent a lower amount of time in the open area when compared to wild-type and heterozygous mice. Similarly, the number of head dipping from the open arcs to the outside of the maze was reduced in Shank $3^{\Delta 4-22}$ homozygous mice. WT, wild-type mice; Het, heterozygous mice; KO, homozygous knock-out mice. $* p<0.05, * * p<0.1, * * * p<0.001$.

typical autism, this subtle behavior can reflect the phenotype of many patients with PMS. Indeed, unlike patients with idiopathic ASD, individuals with PMS show preserved responses to social communication cues (Soorya et al., 2013; Wang et al., 2016a) and roughly equal orient- ing to social versus nonsocial stimuli, despite meeting criteria for ASD. Moreover, the fact that not all individuals with PMS are diagnosed with ASD indicates that animal models for PMS should not necessarily present with strong social behavioral deficits. As the expression and 


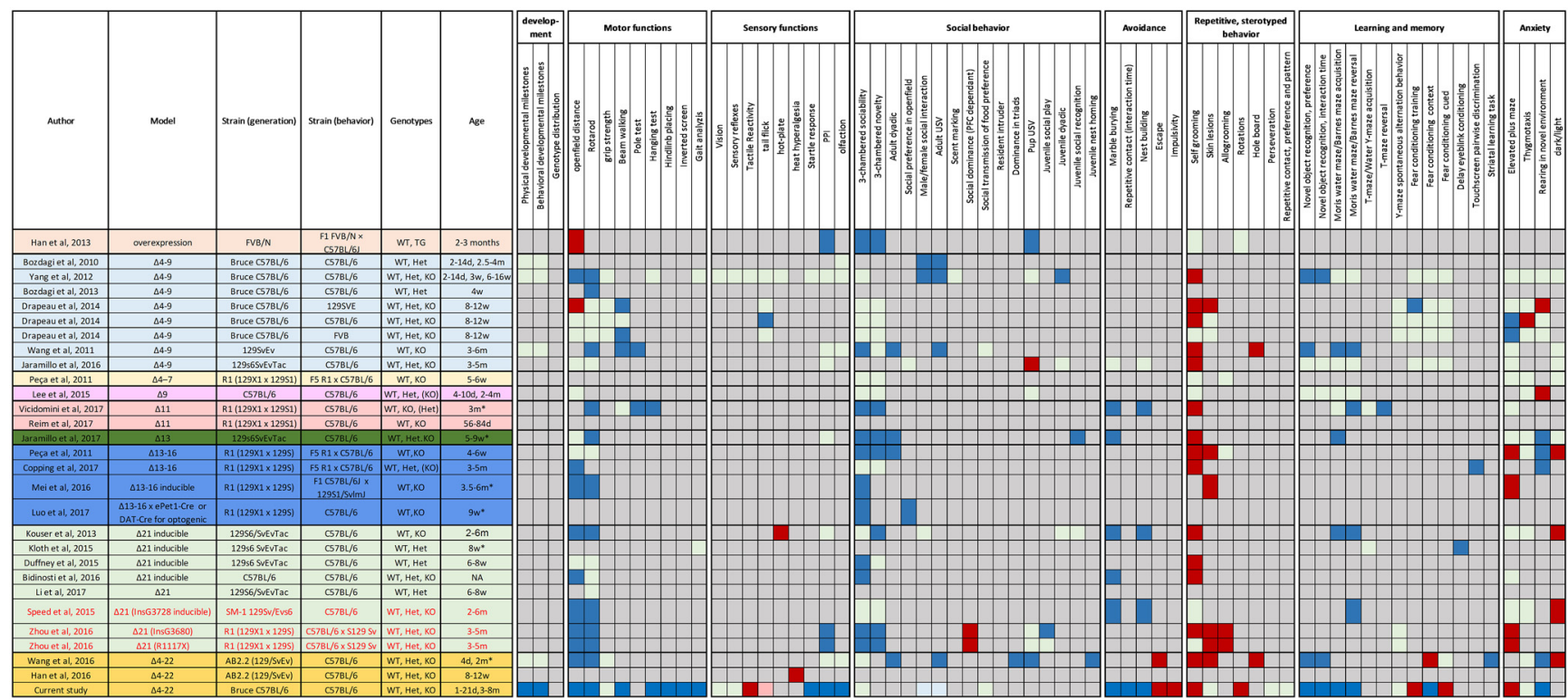

Figure 11. Main features and comorbidities associated with Phelan-McDermid displayed by different mouse models with Shank3 deficits. Green indicates an absence of genotype difference. Blue indicates a decrease of the associated behavior in Shank3-deficient animals. Red indicates an increase of the associated behavior in Shank3-deficient animals. Gray indicates the behavior has not been studied in the corresponding article. Age column: $d=$ days, $w=$ weeks, $m=$ months, $*$ indicates that only the age at the beginning of the testing was provided.

alternative splicing of Shank3 isoforms and even their subcellular distribution has been shown to be cell-type specific, activity dependent, and regionally and developmentally regulated (Wang et al., 2014), these differences also raise the possibility that different Shank3 isoforms could make distinct contributions to the phenotype of PMS and suggests that Shank3c and Shank3d (affected by deletions containing exons 11-16) could be particularly involved in the regulation of social behavior compared to isoforms Shank3a, Shank3b, or Shank3a/b that are disrupted by deletions of exons 4-9. The apparent absence of social deficit in the models with a complete deletion of Shank3 could be explained by the fact that those animals have a strong aversion for objects and be interpreted as an avoidance of the chamber containing the object rather than a real social preference.

One of the strongest phenotype observed in the current study was indeed an active avoidance of inanimate objects. In the novel object recognition test, lack of preference for a novel object had previously been observed in two lines of $\Delta 4-9$ mice (Wang et al., 2011; Yang et al., 2012) but not in a third line (Jaramillo et al., 2016) nor in $\Delta 9$ Shank3-deficient mice (Lee et al., 2015). However, in the present study, homozygous animals had very little interactions with both familiar and novel object making it impossible to properly compare novelty preference. Instead, they mostly spent their time in the corners of the open field away from the objects. Surprisingly, similar avoidance behavior was observed in the marble burying test and in the repetitive novel object contact task. We also observed a strong decrease of direct interactions with the applicator in the olfactory habituation/dishabituation test and a reduction of the quality of the nests build by Shank $3^{\Delta 4-22}$-deficient animals with some mice even leaving the building material fully untouched. Some studies have reported that children with autism respond to novelty with avoidance behaviors and patients with PMS have enhanced reactivity to novel environments and reduced interest for objects. Decrease of marble burying has been consistently been described in other models of Shank3 deficiency as were nest building impairments $(\Delta 11, \Delta 13, \Delta 21$, and exon 21 point mutations; Kouser et al., 2013; Speed et al., 2015; Bidinosti et al., 2016; Jaramillo et al., 2017; Vicidomini et al., 2017). While we have shown that those animals are hypoactive and have significant motor deficits that could impact behavioral assays relying on exploratory locomotion, it is unlikely that this avoidance behavior is attributable to impaired motor activity or poor motivation as homozygous mice have normal pattern of investigation in an empty open field and actively avoid objects or even escape from the cages by jumping out while they will not escape from an empty cage or a cage containing an unfamiliar mouse. Furthermore, the number of escape attempts increased in relation with the number of objects present in the cage. In addition to this escape behavior, a high level of impulsivity was observed for adult homozygous mice in the beam walking test and for both newborn and adult homozygous mice in the negative geotaxis test.

Stereotypies, repetitive behaviors with restricted interests and resistance to change form the second set of core symptoms of ASD. Excessive grooming with or without development of skin lesions is the most commonly observed repetitive behavior in rodents. Repetitive/compulsive grooming has been reported in most of the previously published Shank3 mouse models (Fig. 11) while skin lesions where noticed only in some of them $(\Delta 4-9, \Delta 11$, $\Delta 13-16, \Delta 21$, and point mutations in exon 21; Peça et al., 
2011; Schmeisser et al., 2012; Drapeau et al., 2014; Mei et al., 2016; Zhou et al., 2016) suggesting different levels of severity. The homozygous mice from Wang et al. (2016b) displayed both increased grooming and development of skin lesions. However, in the present study, even if we did occasionally observe some bald patches with or without skin lesions in our oldest animals all genotypes were impacted and group differences where only found for the grooming behavior. Our Shank $3^{\Delta 4-22}$-deficient mice also engaged more frequently in other stereotyped and repetitive behaviors. By contrast, we did not observe any perseveration in the Y-maze nor object or pattern preference in the repetitive novel object contact task. To investigate both cognitive flexibility and insistence on sameness our animals were tested in the Barnes maze. The initial training showed a delay in the acquisition of the task in homozygous mice but after $4 \mathrm{~d}$ of training all genotypes had comparable performances and spent similar amount of time in the target quadrant during a probe test. Mice were then retrained after moving the escape box. Our homozygous mice exhibited impaired cognitive flexibility characterized by a delay in the time needed to learn the new rule and by a similar preference for either the reversal target quadrant or the initial target quadrant during the probe test; heterozygous mice had an intermediate phenotype. This suggests that Shank3 deficiency increases susceptibility to proactive interference, a deficit associated with prefrontal cortex dysfunction. Similar reversal impairments have been published in either the Morris water maze or T-maze in $\Delta 4-9, \Delta 11, \Delta 21$, point mutations, or $\Delta 4-22$ mice (Wang et al., 2011; Kouser et al., 2013; Speed et al., 2015; Wang et al., 2016b; Vicidomini et al., 2017) while other models had comparable results for all genotypes $(\Delta 4-9, \Delta 9$; Yang et al., 2012; Lee et al., 2015; Jaramillo et al., 2016).

Because a majority of patients with SHANK3 mutation/ deletion exhibit some degree of ID, our animals were also tested for short-term memory by examining spontaneous alternation behavior in the $\mathrm{Y}$-maze and for hippocampal or amygdala-dependent memories using contextual and cued fear conditioning. As in other models investigated ( $\Delta 4-9$ and point insertions; Drapeau et al., 2014; Zhou et al., 2016), we found no differences in performance in the Y-maze spontaneous alternation test suggesting normal basic working memory. Neither contextual nor cued memories had been found to be affected by genotype in any of the previously published exon specific models ( $\Delta 4-9$; Yang et al., 2012; Drapeau et al., 2014; Jaramillo et al., 2016) while a small increase of freezing was noticed in $\Delta 4-22$ homozygous mice during contextual recall (Wang et al., 2016b). Interestingly, in our new mouse model, we observed distinct responses to each phase of the testing. While not different at first during the pre-training habituation phase, the level of freezing quickly decreased in wild-type and heterozygous mice but not in the homozygous animals, likely reflecting a higher anxiety level. On presentation of the sound/shock associations, the increase of freezing was significantly more noted in homozygous mice. Remarkably, the opposite was observed during the contextual recall thus demonstrating an impairment of hippocampo dependent memory in homozygous animals, while the same mice displayed increased freezing on the presentation of sounds during the amygdaladependent cued recall.

These region-specific alterations of behavior suggest that different Shank3 deletions could alter different neuronal circuits through the modulation of the expression of different Shank3 isoforms. The Shank3b isoform (present in the $\Delta 21$ mouse models) is expressed at low level throughout the brain, while a regional specificity was observed for the other Shank3 isoforms. Shank3a (absent in all the mouse models) and Shank3e (absent only in $\Delta 21$ and complete gene models) are highly expressed in the striatum but are low in the olfactory bulb and the cerebellum. In contrast, Shank3c (absent in $\Delta 9, \Delta 4-7, \Delta 4-9$, and complete gene models) and Shank3d (absent in $\Delta 13-16$, $\Delta 21$, and complete gene models) are predominantly enriched in the cerebellum (Wang et al., 2014). Specific subcategories of learning and memory behaviors have only been studied in limited number of previous models. Heterozygous $\Delta 21$ mice lacking the cerebellum-specific Shank3c and Shank3d isoforms as well as Shank3e and Shank3f isoforms exhibit impaired eye-blink conditioning, a cerebellar-dependent learning task (Kloth et al., 2015). $\Delta$ 13-16 Shank3-deficient mice are impaired in pairwise visual discrimination learning in the automated touchscreen task depending on normal functions of interconnected cortical and subcortical regions (Copping et al., 2017). Finally, $\Delta 4-22$ homozygous mice have deficits in a striatal-dependent instrumental learning task (Wang et al., 2016b). Further studies examining the extend of impairment of region-specific behaviors will be required to fully understand the relationships between brain circuitry, Shank3 isoforms expression, and behavior.

Altogether, the hyper-reactivity to handling and tactile stimuli, the impulsivity, the object neophobia, the escape behavior, the increased freezing response in the pretraining phase of the fear conditioning and in cued retrieval suggest high levels of anxiety in our mouse model. Hyperactivity and anxiety symptoms are other common features of PMS (Dhar et al., 2010; Soorya et al., 2013; Sarasua et al., 2014a). In previously published models, analysis of anxiety-like behavior measured either elevated mazes, in the open fields or in dark/light emergence boxes have demonstrated a relationship between the targeted isoforms and the manifestations of anxiety likebehavior. While little differences were observed in mouse models with $\Delta 4-9, \Delta 4-7, \Delta 9$, and $\Delta 11$ deletions (Peça et al., 2011; Wang et al., 2011; Schmeisser et al., 2012; Yang et al., 2012; Drapeau et al., 2014; Lee et al., 2015; Jaramillo et al., 2016; Reim et al., 2017; Vicidomini et al., 2017 ) increased levels of anxiety were reported in mice with $\Delta 13, \Delta 13-16$, and $\Delta 21$ deletions or point mutations (Peça et al., 2011; Kouser et al., 2013; Speed et al., 2015; Mei et al., 2016; Zhou et al., 2016; Copping et al., 2017; Jaramillo et al., 2017). Increased levels of anxiety were confirmed in the light-dark emergence test and in the open field in the $\Delta 4-22$ mouse model from Wang et al., $2016 \mathrm{~b}$ and colleagues and in the elevated maze and in the open field in our model. 
In conclusion, our complete Shank $3^{\Delta 4-22}$ mouse line provides a new and improved genetic model for studying mechanisms underlying ASD and PMS and is characterized both by better construct and face validities than previously reported lines of Shank3 mutants. Our in-depth behavioral characterization revealed behavioral features that reflect those observed in PMS and therefore suggest a greater potential as a translational model. Mice with a complete deletion of Shank3 are more severely affected than previously published mouse models with a partial deletion. Both sensory and motor disabilities were detected in neonate and adult mice. Shank $3^{\Delta 4-22}$-deficient mice showed modest deficits in social behavior, reflected in reduced male to female anogenital sniffing and ultrasonic vocalization, but no major deficits in social preference in the three-chambered social interaction task. These findings are consistent with an independently generated mouse model (Wang et al., 2016b). Also in agreement with Wang's study, our Shank $3^{\Delta 4-22}$ mice showed increased anxiety and hyper-reactivity to novel stimuli, increased escape behaviors, and increased repetitive behaviors. Together with the increased freezing behavior in the cued fear conditioning, this suggest a dysregulation of amygdala circuitry that will require further investigation. In addition, our mice displayed impairments in several hippocampal-dependent learning and memory tests as well as cognitive inflexibility, thus recapitulating ID and insistence on sameness observed in autism and in the majority of patients with PMS. Although PMS patients are heterozygous for Shank3 mutations/deletions, most of the previous models have failed to demonstrate any relevant phenotype in heterozygous animals. Here, we were able to observe an intermediate phenotype for heterozygous mice in several of the parameters tested, notably in the open field, rotarod, startle response, escape behavior, reversal probe test, and elevated zero-maze. Heterozygous animals being less affected than their homozygous, we hypothesize that more challenging paradigms, for example by introducing a variable reward probability in tests such as the Barnes maze, would allow us to further highlight differences in heterozygous animals. Past studies have often failed to replicate behavioral phenotype even in models with very similar Shank3 disruption or in different cohorts from the same model. The concordant findings from two independently derived and analyzed Shank3 mouse models, including the comparison of two independent cohorts in our laboratory, demonstrate, for the first time, strong reproducibility and validity for a genetically modified mouse model of PMS, providing a valuable model for further investigations of the neurobiological basis of PMS and ASD.

\section{References}

Barnes CA (1979) Memory deficits associated with senescence: a neurophysiological and behavioral study in the rat. J Comp Physiol Psychol 93:74-104. CrossRef

Benthani F, Tran PN, Currey N, Ng I, Giry-Laterriere M, Carey L, Kohonen-Corish MR, Pangon L (2015) Proteogenomic analysis identifies a novel human SHANK3 isoform. Int J Mol Sci 16:1152211530. CrossRef
Beri S, Tonna N, Menozzi G, Bonaglia MC, Sala C, Giorda R (2007) DNA methylation regulates tissue-specific expression of Shank3. J Neurochem 101:1380-1391. CrossRef

Betancur C, Buxbaum JD (2013) SHANK3 haploinsufficiency: a "common" but underdiagnosed highly penetrant monogenic cause of autism spectrum disorders. Mol Autism 4:17. CrossRef

Bidinosti M, Botta P, Krüttner S, Proenca CC, Stoehr N, Bernhard M, Fruh I, Mueller M, Bonenfant D, Voshol H, Carbone W, Neal SJ, McTighe SM, Roma G, Dolmetsch RE, Porter JA, Caroni P, Bouwmeester T, Lüthi A, Galimberti I (2016) CLK2 inhibition ameliorates autistic features associated with SHANK3 deficiency. Science 351:1199-1203. CrossRef

Bonaglia MC, Giorda R, Beri S, De Agostini C, Novara F, Fichera M, Grillo L, Galesi O, Vetro A, Ciccone R, Bonati MT, Giglio S, Guerrini R, Osimani S, Marelli S, Zucca C, Grasso R, Borgatti R, Mani E, Motta C, et al. (2011) Molecular mechanisms generating and stabilizing terminal $22 q 13$ deletions in 44 subjects with Phelan/McDermid syndrome. PLoS Genet 7:e1002173. CrossRef

Bozdagi O, Sakurai T, Papapetrou D, Wang X, Dickstein DL, Takahashi N, Kajiwara Y, Yang M, Katz AM, Scattoni ML, Harris MJ, Saxena R, Silverman JL, Crawley JN, Zhou Q, Hof PR, Buxbaum JD (2010) Haploinsufficiency of the autism-associated Shank3 gene leads to deficits in synaptic function, social interaction, and social communication. Mol Autism 1:15. CrossRef

Carter RJ, Morton J, Dunnett SB (2001) Motor coordination and balance in rodents. Curr Protoc Neurosci Chapter 8:Unit 8.12.

Copping NA, Berg EL, Foley GM, Schaffler MD, Onaga BL, Buscher N, Silverman JL, Yang M (2017) Touchscreen learning deficits and normal social approach behavior in the Shank3B model of PhelanMcDermid syndrome and autism. Neuroscience 345:155-165. CrossRef

Cusmano-Ozog K, Manning MA, Hoyme HE (2007) 22q13.3 deletion syndrome: a recognizable malformation syndrome associated with marked speech and language delay. Am J Med Genet C Semin Med Genet 145C:393-398. CrossRef

Deacon RM (2006) Assessing nest building in mice. Nat Protoc 1:1117-1119. CrossRef Medline

De Rubeis S, Siper PM, Durkin A, Weissman J, Muratet F, Halpern D, Trelles MDP, Frank Y, Lozano R, Wang AT, Holder JL Jr, Betancur C, Buxbaum JD, Kolevzon A (2018) Delineation of the genetic and clinical spectrum of Phelan-McDermid syndrome caused by SHANK3 point mutations. Mol Autism 9:31. CrossRef

Dhar SU, del Gaudio D, German JR, Peters SU, Ou Z, Bader PI, Berg JS, Blazo M, Brown CW, Graham BH, Grebe TA, Lalani S, Irons M, Sparagana S, Williams M, Phillips JA 3rd, Beaudet AL, Stankiewicz P, Patel A, Cheung SW, et al. (2010) 22q13.3 deletion syndrome: clinical and molecular analysis using array CGH. Am J Med Genet A 152A:573-581. CrossRef

Drapeau E, Dorr NP, Elder GA, Buxbaum JD (2014) Absence of strong strain effects in behavioral analyses of Shank3-deficient mice. Dis Model Mech 7:667-681. CrossRef Medline

Duffney LJ, Zhong P, Wei J, Matas E, Cheng J, Qin L, Ma K, Dietz DM, Kajiwara Y, Buxbaum JD, Yan Z (2015) Autism-like deficits in Shank3-deficient mice are rescued by targeting actin regulators. Cell Rep 11:1400-1413. CrossRef

Durand CM, Betancur C, Boeckers TM, Bockmann J, Chaste P, Fauchereau F, Nygren G, Rastam M, Gillberg IC, Anckarsäter H, Sponheim E, Goubran-Botros H, Delorme R, Chabane N, MourenSimeoni MC, de Mas P, Bieth E, Rogé B, Héron D, Burglen L, et al. (2007) Mutations in the gene encoding the synaptic scaffolding protein SHANK3 are associated with autism spectrum disorders. Nat Genet 39:25-27. CrossRef

Fox WM (1965) Reflex-ontogeny and behavioural development of the mouse. Anim Behav 13:234-241. Medline

Harony-Nicolas H, Kay M, Hoffmann JD, Klein ME, Bozdagi-Gunal O, Riad M, Daskalakis NP, Sonar S, Castillo PE, Hof PR, Shapiro ML, Baxter MG, Wagner S, Buxbaum JD (2017) Oxytocin improves behavioral and electrophysiological deficits in a novel Shank3deficient rat. Elife 6. CrossRef 
Heyser CJ (2004) Assessment of developmental milestones in rodents. Curr Protoc Neurosci Chapter 8:Unit 8.18.

Jaramillo TC, Speed HE, Xuan Z, Reimers JM, Liu S, Powell CM (2016) Altered striatal synaptic function and abnormal behaviour in Shank3 Exon4-9 deletion mouse model of autism. Autism Res 9:350-375. CrossRef

Jaramillo TC, Speed HE, Xuan Z, Reimers JM, Escamilla CO, Weaver TP, Liu S, Filonova I, Powell CM (2017) Novel Shank3 mutant exhibits behaviors with face validity for autism and altered striatal and hippocampal function. Autism Res 10:42-65. CrossRef

Klintwall L, Holm A, Eriksson M, Carlsson LH, Olsson MB, Hedvall A, Gillberg C, Fernell E (2011) Sensory abnormalities in autism. A brief report. Res Dev Disabil 32:795-800. CrossRef Medline

Kloth AD, Badura A, Li A, Cherskov A, Connolly SG, Giovannucci A, Bangash MA, Grasselli G, Peñagarikano O, Piochon C, Tsai PT, Geschwind DH, Hansel C, Sahin M, Takumi T, Worley PF, Wang SS (2015) Cerebellar associative sensory learning defects in five mouse autism models. Elife 4:e06085. CrossRef

Kouser M, Speed HE, Dewey CM, Reimers JM, Widman AJ, Gupta N, Liu S, Jaramillo TC, Bangash M, Xiao B, Worley PF, Powell CM (2013) Loss of predominant Shank3 isoforms results in hippocampus-dependent impairments in behavior and synaptic transmission. J Neurosci 33:18448-18468. CrossRef

Leblond CS, Nava C, Polge A, Gauthier J, Huguet G, Lumbroso S, Giuliano F, Stordeur C, Depienne C, Mouzat K, Pinto D, Howe J, Lemière N, Durand CM, Guibert J, Ey E, Toro R, Peyre H, Mathieu A, Amsellem F, et al. (2014) Meta-analysis of SHANK mutations in autism spectrum disorders: a gradient of severity in cognitive impairments. PLoS Genet 10:e1004580. CrossRef

Lee J, Chung C, Ha S, Lee D, Kim DY, Kim H, Kim E (2015) Shank3-mutant mice lacking exon 9 show altered excitation/inhibition balance, enhanced rearing, and spatial memory deficit. Front Cell Neurosci 9:94. CrossRef

Li C, Schaefer M, Gray C, Yang Y, Furmanski O, Liu S, Worley P, Mintz CD, Tao F, Johns RA (2017) Sensitivity to isoflurane anesthesia increases in autism spectrum disorder Shank3+/c mutant mouse model. Neurotoxicol Teratol 60:69-74. CrossRef

Luciani JJ, de Mas P, Depetris D, Mignon-Ravix C, Bottani A, Prieur M, Jonveaux P, Philippe A, Bourrouillou G, de Martinville B, Delobel B, Vallee L, Croquette MF, Mattei MG (2003) Telomeric 22q13 deletions resulting from rings, simple deletions, and translocations: cytogenetic, molecular, and clinical analyses of 32 new observations. J Med Genet 40:690-696. CrossRef

Luo J, Feng Q, Wei L, Luo M (2017) Optogenetic activation of dorsal raphe neurons rescues the autistic-like social deficits in Shank3 knockout mice. Cell Res 27:950-953.

Maunakea AK, Nagarajan RP, Bilenky M, Ballinger TJ, D'Souza C, Fouse SD, Johnson BE, Hong C, Nielsen C, Zhao Y, Turecki G, Delaney A, Varhol R, Thiessen N, Shchors K, Heine VM, Rowitch DH, Xing X, Fiore C, Schillebeeckx M, et al. (2010) Conserved role of intragenic DNA methylation in regulating alternative promoters. Nature 466:253-257. CrossRef

Mei Y, Monteiro P, Zhou Y, Kim JA, Gao X, Fu Z, Feng G (2016) Adult restoration of Shank3 expression rescues selective autistic-like phenotypes. Nature 530:481-484. CrossRef Medline

Moessner R, Marshall CR, Sutcliffe JS, Skaug J, Pinto D, Vincent J, Zwaigenbaum L, Fernandez B, Roberts W, Szatmari P, Scherer SW (2007) Contribution of SHANK3 mutations to autism spectrum disorder. Am J Hum Genet 81:1289-1297. CrossRef Medline

Nadler JJ, Moy SS, Dold G, Trang D, Simmons N, Perez A, Young NB, Barbaro RP, Piven J, Magnuson TR, Crawley JN (2004) Automated apparatus for quantitation of social approach behaviors in mice. Genes Brain Behav 3:303-314. CrossRef

Pearson BL, Pobbe RL, Defensor EB, Oasay L, Bolivar VJ, Blanchard DC, Blanchard RJ (2011) Motor and cognitive stereotypies in the BTBR T+tf/J mouse model of autism. Genes Brain Behav 10:228235. CrossRef

Peça J, Feliciano C, Ting JT, Wang W, Wells MF, Venkatraman TN, Lascola CD, Fu Z, Feng G (2011) Shank3 mutant mice display autistic-like behaviours and striatal dysfunction. Nature 472:437442. CrossRef

Phelan K, Betancur C (2011) Clinical utility gene card for: deletion $22 q 13$ syndrome. Eur J Hum Genet 19.

Phelan K, McDermid HE (2012) The 22q13.3 deletion syndrome (Phelan-McDermid syndrome). Mol Syndromol 2:186-201. Medline Medline

Reim D, Distler U, Halbedl S, Verpelli C, Sala C, Bockmann J, Tenzer S, Boeckers TM, Schmeisser MJ (2017) Proteomic analysis of post-synaptic density fractions from Shank3 mutant mice reveals brain region specific changes relevant to autism spectrum disorder. Front Mol Neurosci 10:26. CrossRef

Sarasua SM, Boccuto L, Sharp JL, Dwivedi A, Chen CF, Rollins JD, Rogers RC, Phelan K, DuPont BR (2014a) Clinical and genomic evaluation of 201 patients with Phelan-McDermid syndrome. Hum Genet 133:847-859. CrossRef Medline

Sarasua SM, Dwivedi A, Boccuto L, Chen CF, Sharp JL, Rollins JD, Collins JS, Rogers RC, Phelan K, DuPont BR (2014b) $22 q 13.2 q 13.32$ genomic regions associated with severity of speech delay, developmental delay, and physical features in Phelan-McDermid syndrome. Genet Med 16:318-328. CrossRef

Scattoni ML, Ricceri L, Crawley JN (2011) Unusual repertoire of vocalizations in adult BTBR $\mathrm{T}+\mathrm{tf} / \mathrm{J}$ mice during three types of social encounters. Genes Brain Behav 10:44-56. CrossRef Medline

Schmeisser MJ, Ey E, Wegener S, Bockmann J, Stempel AV, Kuebler A, Janssen AL, Udvardi PT, Shiban E, Spilker C, Balschun D, Skryabin BV, Dieck St, Smalla KH, Montag D, Leblond CS, Faure P, Torquet N, Le Sourd AM, Toro R, et al. (2012) Autistic-like behaviours and hyperactivity in mice lacking ProSAP1/Shank2. Nature 486:256-260. CrossRef Medline

Soorya L, Kolevzon A, Zweifach J, Lim T, Dobry Y, Schwartz L, Frank Y, Wang AT, Cai G, Parkhomenko E, Halpern D, Grodberg D, Angarita B, Willner JP, Yang A, Canitano R, Chaplin W, Betancur C, Buxbaum JD (2013) Prospective investigation of autism and genotype-phenotype correlations in $22 \mathrm{q} 13$ deletion syndrome and SHANK3 deficiency. Mol Autism 4:18. CrossRef

Speed HE, Kouser M, Xuan Z, Reimers JM, Ochoa CF, Gupta N, Liu S, Powell CM (2015) Autism-associated insertion mutation (InsG) of Shank3 exon 21 causes impaired synaptic transmission and behavioral deficits. J Neurosci 35:9648-9665. CrossRef

Steinbach JM, Garza ET, Ryan BC (2016) Novel object exploration as a potential assay for higher order repetitive behaviors in mice. J Vis Exp

Sykes NH, Toma C, Wilson N, Volpi EV, Sousa I, Pagnamenta AT, Tancredi R, Battaglia A, Maestrini E, Bailey AJ, Monaco AP; International Molecular Genetic Study of Autism Consortium (IMGSAC) (2009) Copy number variation and association analysis of SHANK3 as a candidate gene for autism in the IMGSAC collection. Eur $\mathrm{J}$ Hum Genet 17:1347-1353. CrossRef Medline

Thomas A, Burant A, Bui N, Graham D, Yuva-Paylor LA, Paylor R (2009) Marble burying reflects a repetitive and perseverative behavior more than novelty-induced anxiety. Psychopharmacology (Berl) 204:361-373. CrossRef

Vicidomini C, Ponzoni L, Lim D, Schmeisser MJ, Reim D, Morello N, Orellana D, Tozzi A, Durante V, Scalmani P, Mantegazza M, Genazzani AA, Giustetto M, Sala M, Calabresi P, Boeckers TM, Sala C, Verpelli C (2017) Pharmacological enhancement of mGlu5 receptors rescues behavioral deficits in SHANK3 knock-out mice. Mol Psychiatry 22:784.

Waga C, Asano H, Sanagi T, Suzuki E, Nakamura Y, Tsuchiya A, Itoh M, Goto Y, Kohsaka S, Uchino S (2014) Identification of two novel Shank3 transcripts in the developing mouse neocortex. J Neurochem 128:280-293. Medline

Wang AT, Lim T, Jamison J, Bush L, Soorya LV, Tavassoli T, Siper PM, Buxbaum JD, Kolevzon A (2016a) Neural selectivity for communicative auditory signals in Phelan-McDermid syndrome. J Neurodev Disord 8:5. CrossRef 
Wang X, McCoy PA, Rodriguiz RM, Pan Y, Je HS, Roberts AC, Kim CJ, Berrios J, Colvin JS, Bousquet-Moore D, Lorenzo I, Wu G, Weinberg RJ, Ehlers MD, Philpot BD, Beaudet AL, Wetsel WC, Jiang YH (2011) Synaptic dysfunction and abnormal behaviors in mice lacking major isoforms of Shank3. Hum Mol Genet 20:3093-3108. CrossRef

Wang X, Xu Q, Bey AL, Lee Y, Jiang YH (2014) Transcriptional and functional complexity of Shank3 provides a molecular framework to understand the phenotypic heterogeneity of SHANK3 causing autism and Shank3 mutant mice. Mol Autism 5:30. CrossRef

Wang X, Bey AL, Katz BM, Badea A, Kim N, David LK, Duffney LJ, Kumar S, Mague SD, Hulbert SW, Dutta N, Hayrapetyan V, Yu C, Gaidis E, Zhao S, Ding JD, Xu Q, Chung L, Rodriguiz RM, Wang F, et al. (2016b) Altered mGluR5-Homer scaffolds and corticostriatal connectivity in a Shank3 complete knockout model of autism. Nat Commun 7:11459. CrossRef

Wrenn CC, Harris AP, Saavedra MC, Crawley JN (2003) Social transmission of food preference in mice: methodology and application to galanin-overexpressing transgenic mice. Behav Neurosci 117:21-31. CrossRef
Yang M, Crawley JN (2009) Simple behavioral assessment of mouse olfaction. Curr Protoc Neurosci Chapter 8:Unit 8.24.

Yang M, Bozdagi O, Scattoni ML, Wöhr M, Roullet FI, Katz AM, Abrams DN, Kalikhman D, Simon H, Woldeyohannes L, Zhang JY, Harris MJ, Saxena R, Silverman JL, Buxbaum JD, Crawley JN (2012) Reduced excitatory neurotransmission and mild autismrelevant phenotypes in adolescent Shank3 null mutant mice. $J$ Neurosci 32:6525-6541. CrossRef

Zhou Y, Kaiser T, Monteiro P, Zhang X, Van der Goes MS, Wang D, Barak B, Zeng M, Li C, Lu C, Wells M, Amaya A, Nguyen S, Lewis M, Sanjana N, Zhou Y, Zhang M, Zhang F, Fu Z, Feng G (2016) Mice with Shank3 mutations associated with ASD and schizophrenia display both shared and distinct defects. Neuron 89:147-162. CrossRef

Zhu L, Wang X, Li XL, Towers A, Cao X, Wang P, Bowman R, Yang $\mathrm{H}$, Goldstein J, Li YJ, et al. (2014) Epigenetic dysregulation of SHANK3 in brain tissues from individuals with autism spectrum disorders. Hum Mol Genet 23:1563-1578. CrossRef Medline 